\title{
Ausgewählte narrative Vermittlungsvorkommen von Erzählungen zur imagefördernden (Selbst)Darstellung
}

\author{
„Etwas wissenschaftlich behandeln, heißt, Ordnung zu \\ stiften. Und Ordnung stiften wiederum heißt, in der Fülle \\ des realen Einzelnen die Wiederkehr von etwas \\ feststellen, was dieses Einzelne übergreift.“ \\ (Hans Hörmann, nach Gaede 1992: 8)
}

Im Sinne des einführenden Zitats behandle ich in diesem Kapitel, auf welche Art und Weise Personen auf verschiedenen Internetplattformen Erzählungen gestalten, die im Zuge einer imagefördernden (S)D von Unternehmen entstehen. Hierzu bündle ich ausgewählte und wiederkehrende Realisierungsmöglichkeiten anhand bestimmter Gemeinsamkeiten. Ich fasse die verschiedenen Möglichkeiten unter dem Überbegriff narratives Vermittlungsvorkommen. Präziser gesagt, drückt der Terminus ,Vermittlung' aus, dass etwas weitergegeben, kommuniziert wird, während der Begriff ,Vorkommen“ auf konkrete multimodale Realisierungsmöglichkeiten verweist. Im Kontext der Arbeit sehe ich diese Vermittlungsvorkommen als das Ergebnis von unternehmensbezogenen Praktiken, deren vornehmlich sprachlich-kommunikative Formen eine Ressource für die Handlung ,(S)D` sind. Der Terminus ,narrativ ' grenzt das Vorkommen dahingehend ein, dass es für Erzählungen spezifische Eigenschaften aufweist (Abschn. 5.2 bis 5.4). Das kommunizierte Etwas bezieht sich wiederum im Kontext der Arbeit auf erzählte Ereignisse, die die (S)D von Unternehmen fördern (Abschn. 6.2.1) und nicht für 
werbliche Informationen im engeren Sinne der Produktwerbung (z. B. ProduktWerbefilme, Werbebanner u. a.) produziert werden ${ }^{1}$. Die Erzählungen transportieren (S)D, die auf ein positives Image zielen. Dieses Image beruht darauf, dass Unternehmensakteure ein zuvor konstruiertes Selbstbild im Sinne einer Corporate Identity in die Erzählungen projizieren, um ein möglichst gutes Bild des Unternehmens nach außen hervorzurufen. Unter diesem so intendierten Fremdbild im Sinne der Betriebs- und Wirtschaftswissenschaften ist der zugrundegelegte Imagebegriff verortet $^{2}$ (vgl. Schuster (2000), nach Burel 2015: 82). Von Bedeutung für den hiesigen Kontext ist, dass Unternehmen versuchen, solch ein gewünschtes Selbstbild zu vermitteln, indem sie Rezipient/innen extralinguistisch gewonnene Eindrücken aus ihrer „Wirklichkeit“ (Burel 2015: 82) anbieten: Eine zunehmend beliebte Option hierfür ist der narrative Kommunikationsmodus. Im Rahmen dieser Arbeit unterstelle ich den erhobenen Erzählungen, dass sie in einem hohen Maße imagefördernde (S)D inkludieren. Welche Möglichkeiten Unternehmen zur (S)D im Rahmen ihrer Erzählungen aufgreifen, wurde im Detail in Kapitel sieben (Narrativ gestützte Typen zur imagefördernden (Selbst)Darstellung) behandelt. Das vorliegende Kapitel erfasst wiederkehrende Erscheinungsformen des erhobenen narrativen Vermittlungsvorkommens zur imagefördernden (S)D von Unternehmen auf verschiedenen Internetplattformen anhand von charakteristischen Merkmale und beschreibt sie als narrative Praktiken.

Die vorliegende Untersuchung zeigt, dass die technischen Gegebenheiten der unterschiedlichen Internetplattformen und die Nutzerkonventionen das narrative Vermittlungsvorkommen zwar beeinflussen, es deshalb jedoch nicht ausschließlich an eine Plattform gebunden sein muss (bspw. erscheinen diskontinuierliche Erzählungen sowohl auf Facebook, Twitter oder auf Xing): Das narrative Vermittlungsvorkommen tritt also unabhängig von einer Internetplattform und dadurch auch in Variationen auf. Deswegen gehe ich in der folgenden Ergebnisdarstellung so vor, dass ich die herausgearbeiteten Merkmale der Vermittlungsvorkommen anhand von Beispielen konkretisiere, die den Internetplattformen entnommen

\footnotetext{
${ }^{1}$ Neben den in dieser Arbeit untersuchten Erzählungen (uRE) bestehen durchaus auch andere Erzählungen von Unternehmen. Ebenfalls ist nicht ausgeschlossen, dass das hier zu beschreibende Vermittlungsvorkommen unabhängig von der fokussierten Form der (S)D auftritt. Das betrifft $\mathrm{u}$. a. bestimmte Themenbereiche innerhalb von Corporate Blogs wie beispielsweise bei Edeka „Meine Lieblingsrezepte“ oder bei Daimler „Technologie \& Innovation“, ,smart“, „Daimler Buse“ u. a. In diesen Beiträgen treten auch Erzählungen auf, die jedoch das eigentliche Produkt in Form von Erlebnisnarrativen bewerben und eine (klare) Kaufaufforderung kommunizieren.

${ }^{2}$ Eine ausführliche Diskussion über die Verwendung des Imagebegriffs findet sich in Abschn. 1.1.
} 
sind, auf denen sie überwiegend vorkommen. Es handelt sich um ausgewählte Beispiele, die die erhobenen Merkmale besonders treffend abbilden. Daher sind die Aussagen und Muster des narrativen Vermittlungsvorkommens grundsätzlich innerhalb des Korpus anzusetzen. Es wird nicht behauptet, dass es sich bei dem Vorkommen um spezifische Textsorten handle. Da es jedoch einfacher ist über etwas zu schreiben, das man beim Namen nennt, habe ich wiederkehrende Muster nahe an ihren Spezifika unter Arbeitsbegriffen zusammengefasst.

Narrative Vermittlungsvorkommen, die in den vorliegenden Daten besonders häufig auf bestimmten Internetplattformen auftreten, können in erster Linie in „große“ und „kleine“ narrative Projekte sortiert werden. Auf die „kleinen“ narrativen Vermittlungsvorkommen gehe ich detailliert ein, da die linguistische Forschung sie $\mathrm{m}$. W. im Kontext von Unternehmen nur marginal im Blick hat. Die „großen“ narrativen Vermittlungsformen werden im Vergleich dazu sowohl von der Linguistik als auch von anderen Fachdisziplinen deutlich häufiger berücksichtigt. So kann ich in diesen Fällen auf bereits vorhandene linguistische Forschung zurückgreifen und meine Untersuchung auf Bereiche richten, die ich im Zusammenhang mit diesen Erzählungen von Unternehmen (uRE) für besonders beachtenswert halte. Folgende narrative Vermittlungsvorkommen treten in den vorliegenden Daten besonders häufig auf den dazu benannten Internetplattformen auf:

„Große“ Projekte narrativer Vermittlungsvorkommen

1) Gründungserzählung: U.-Homepages inklusive U.-Historyblogs

2) Corporate Blogerzählungen: Corporate Blogs

„Kleine“ Projekte narrativer Vermittlungsvorkommen

3) Bimodale Kompakterzählungen: Facebook, Xing

4) Diskontinuierliche Erzählungen: Twitter, Facebook

Das Ziel dieses Kapitels ist es nicht, diese Variationen erschöpfend darzustellen, sondern sie mithilfe grundlegender Charakteristika als narrative Praktiken im Kontext von Unternehmen zu erfassen, zu bündeln und zu beschreiben. Charakteristische Beispiele veranschaulichen die Merkmale, um das vorgefundene Vermittlungsvorkommen zu begründen. Ausgehend von den zuvor beschriebenen Kategorien (Abschn. 6.2.2) stehen vier grundlegende Fragen im Mittelpunkt der Beschreibung: 
1) Präferieren die untersuchten Unternehmen auf den verschiedenen Internetplattformen bestimmte Themenfelder für das realisierte, imagefördernde narrative Vermittlungsvorkommen?

2) Wer erzählt die uRE unter welcher Perspektive?

3) Welche (text-)strukturellen Merkmale sind für die unterschiedlichen narrativen Vermittlungsvorkommen konstitutiv, wie sind die uRE im Anwendungskontext realisiert?

4) Welchen Beitrag leisten spezifische Text-, Bild- und Bewegtbildkomponenten für die uRE?

\subsection{Gründungserzählung}

Auf der U.-Homepage unter Navigationsbezeichnungen wie Unternehmensgeschichte, Geschichte oder History finden sich Gründungserzählungen von Unternehmen, die eine narrative Aufbereitung der Unternehmensherkunft und/oder -vergangenheit annehmen lassen. Da die Produzent/innen von Gründungserzählungen in der Regel unbenannt bleiben, ist immer das Unternehmen der Absender, der die uRE sowohl für interne als auch für externe Interessierte verfassen lässt. Schach $(2016,2017)$ oder auch Krüger (2015) summieren die Erzählungen als PR-Text unter den Bereich der Public Relations (siehe hierzu auch Abschn. 7.1.1.1). Bezeichnend für diese Texte ist ein hohes Maß intentionaler Kommunikation, das sich nach ,den strategischen Grundüberlegungen zur Positionierung eines Unternehmens richtet" (Schach 2016: 82). Daher sind sie als Quelle für narrative Praktiken von Unternehmen zur imagefördernden (S)D unerlässlich (siehe bspw. Abschn. 8.3 Grundtyp 1: das traditionell verankerte Unternehmen). Die Bedeutung der Gründungserzählung für ein Unternehmen erschließt sich, wenn man sich vor Augen führt, dass sie sozusagen ,in narrativer Form die DNA“ (Schach 2016: 33) eines Unternehmens bilden. Die Unternehmensanfänge dienen den Autor/innen als Vorlage dafür, um auf realen Geschehnissen beruhende Missionen, Werte oder Visionen als über die Zeit gewachsene Stärken und Errungenschaften zu präsentieren und im Hier-und-Jetzt zu verankern. Dadurch sind sie als „,,starkes historisches Kompetenzzentrum“ und Pool für verwurzelte Werte und Traditionen“ (Bühler/Dürig 2008: 9, nach Schach 2016: 33) eine Grundlage, auf der Unternehmen versuchen sich sehr ,,persönlich" von anderen Unternehmen abzugrenzen und als einzigartig zu stilisieren. Entsprechend ist eine Gründungserzählung ,als Fundament der kommunikativen Außendarstellung für viele Unternehmen immer wichtiger" (Schach 2016: 65). 
In der Fachliteratur wird diesbezüglich von der Basisgeschichte oder Core-Story eines Unternehmens gesprochen (Schach 2016: 29 ff.; Huck-Sandhu 2014: 664; Fog et al. 2010: 55; Loebbert 2003: 57 ff.), die die „Unternehmensessenz“ (Huck-Sandhu 2014: 664), die „Unternehmenspersönlichkeit“ (Schach 2017: 63) beinhaltet.

Gründungserzählungen können als Unternehmensgeschichte oder als Gründererzählung umgesetzt werden. Während die Unternehmensgeschichte sich mit der historischen Unternehmensentwicklung im Allgemeinen auseinandersetzt (Schach 2016: 16), befassen sich die Erzähltextinhalte im Falle der Gründererzählung mit Geschichten rund um die Gründerpersönlichkeiten. Dies geschieht mit dem Ziel, einen Mythos zu etablieren. Er ,hilft Wertsetzungen einer Marke auf eine Kernidee $\mathrm{zu}$ verdichten, verschiedene Erfahrungen $\mathrm{zu}$ integrieren, eine bildhafte Erlebnisdimension und Sympathie hervorzurufen“ (Schach 2016: 33). Mithilfe des Mythos soll eine Entwicklung in der Zeit als geschichtliche Leistung in die Gegenwart eines Unternehmens transferiert und sowohl für die Gegenwart als auch für die Zukunft als richtungsweisend etabliert werden (vgl. ebd., siehe auch Abschn. 7.2.2.2). Sammer (2014: 71) unterscheidet hierbei zwei Grundmuster:

1. Mythen, in denen Personen als charismatische Visionäre verewigt werden.

2. Mythen, in denen die Umstände der Unternehmensgründung im Vordergrund stehen.

Schach hat unter einer textlinguistischen Perspektive die Unternehmensgeschichten der DAX-30-Unternehmen untersucht. Ihre Ergebnisse finde ich in den erhobenen Daten wieder. Aus diesem Grund werde ich im folgenden Unterkapitel ihre Untersuchungsergebnisse vorstellen und mit den vorliegenden Daten in Beziehung setzen. Im Anschluss daran analysiere ich zwei „Extrembeispiele“ aus den vorliegenden Daten vor diesem Hintergrund und betrachte sie unter einer gesamt-narratologischen Perspektive.

\subsubsection{Gründungserzählungen der DAX-30-Unternhemen nach Annika Schach (2016)}

Schach (2016) untersucht Unternehmens- und Gründererzählungen der DAX-30Unternehmen, die sie in Anlehnung an Sammer (2014: 55) unter dem Begriff ,Unternehmensgeschichten“ erfasst. Initial erwartet sie im Zusammenhang mit dem Corporate Storytelling eine deutlich narrative Umsetzung, da die Methode 
des Storytelling insbesondere in den Anwendungsbereichen der Public Relations im Trend liege. Unter Corporate Storytelling versteht sie nach Krüger (2015)

„[...] eine Kommunikationsoperation des Public Relations-Managements gewinnorientierter Organisationen des Wirtschaftssystems. [...] Das Public RelationsManagement operiert dabei in einem erzählenden Kommunikationsmodus und kommuniziert narrative Selbstdarstellungen in der Form von Corporate Storys. Diese Corporate Storys weisen tradierte Elemente und Strukturen von Erzählungen wie Akteure, Ereignisse, Orte, zeitliche und logische Verläufe und Handlungsmuster auf, die das Identitäts-, Aufmerksamkeits- und Deutungsmanagement der Organisation unterstützen.“ (Krüger 2015: 100)

Die Motivation der Autorin für die Analyse beruht auf ihrer Wahrnehmung, dass „,[b]ei der bisherigen Beurteilung von Texten in Bezug auf das Storytelling und in der Beschäftigung mit diesem Trend, [...] die Text-Ebene im Vergleich zur Inhaltsebene eher vernachlässigt [wurde]" (Schach 2016: 6). Nur die Inhaltsebene zu untersuchen - so die Autorin - greife zu kurz, denn auch eine gute Geschichte könne schlecht erzählt werden (Schach 2017: 67). Ernüchtert stellt sie fest, dass Unternehmen zwar ihre Vergangenheit als ,Geschichte ' ankündigen, dabei jedoch die narrativen Möglichkeiten bei Weitem nicht ausschöpfen. Vielmehr veröffentlichten sie ihre Geschichte im Stil einer Chronik bzw. eines Protokolls. Dabei unterschieden sich die einzelnen Texte so deutlich voneinander, dass sich keine klaren Merkmale herausstellen lassen, aufgrund derer man von einer Textsorte ,Unternehmensgeschichte" sprechen könne (Schach 2017: 70).

Für ihre Untersuchung entwickelt Schach ein Dimensionenmodell, das auf der Mehrebenenanalyse von Textsorten basiert. Die zugrunde gelegten Dimensionen umfassen den Kontext, die Textrealisierung (Typographie, Text-Bild-Relation), die Themenentfaltung (Textthema und -hierarchie) und die Sprache (Textstil, sprachliche Mittel) (Schach 2016: 73). Ihr Ziel ist es Vertextungsstrategien von Unternehmensgeschichten herauszuarbeiten. Fragestellungen, denen Schach diesbezüglich nachgeht und die für das vorliegende Kapitel von besonderem Interesse sind, untersuchen, (1) inwiefern Unternehmen ihre Historie narrativ vermitteln, (2) ob sich narrative Typen identifizieren lassen und (3) in welcher Beziehung Text und Bild in den Unternehmensgeschichten der DAX-30-Unternehmen stehen. Das Korpus der Autorin umfasst 29 Unternehmensgeschichten. Fünf der von mir untersuchten Unternehmen gehören ebenfalls zu den DAX-30-Unternehmen ${ }^{3}$. Die Analyse der verbleibenden fünf Unternehmen ${ }^{4}$ zeigt, dass die Ergebnisse

\footnotetext{
${ }^{3}$ Daimler, Dt. Telekom, E.ON, Siemens, Volkswagen.

${ }^{4}$ Telefonica Dtl., EnBW, Bosch, Rewe, Edeka.
} 
auf sie ebenfalls zutreffen. Daher fasse ich im Folgenden bestimmte Ergebnisse von Schach zusammen, die im Rahmen der hier zugrunde gelegten Beschreibungsebenen für das narrative Vermittlungsvorkommen der zehn untersuchten Gründungserzählungen relevant sind. Dabei handelt es sich zum einen um die hier zugrunde gelegte Beschreibungsebene ,narrative Themenentfaltung'. Innerhalb der Ergebnisse von Schach bildet sie sich in den ermittelten ,Textthemen“ (entspr. dem Merkmal ,thematische Ausarbeitung') und den ,narrativen Typen " (entspr. dem Merkmal ,inhaltliche Struktur') ab. Zum anderen sind für die Beschreibungsebene ,(Text-)Realisierung ‘ die Ergebnisse hinsichtlich der Text-Bild-Relationen übertragbar. Da die Grundlage jeder Erzählung das Textthema ist, gehe ich auf diese Ergebnisse zuerst ein.

\subsubsection{Kategorisierung der Textthemen}

Das Textthema fasst Schach unter der textlinguistischen Perspektive als ,die kleinstmögliche Kurzfassung des Textinhalts“ (Schach 2016: 98). Sie geht dabei ebenso, wie ich das in der vorliegenden Analyse in Abschnitt 6.2.3 erläutert habe, von dem alltagssprachlichen Begriffsverständnis und der Erhebung anhand von Paraphrasen nach Brinker et al. (2014: 52 f.) aus. Dadurch identifiziert die Autorin drei verschiedene Möglichkeiten, das Textthema in Gründungserzählungen zu gestalten (Schach 2016: 99):

1. Das Unternehmen als ausschließliches Textthema: In dieser Option steht die Entwicklung des Unternehmens im Mittelpunkt, während auf die Gründer marginal oder gar nicht Bezug genommen wird.

2. Das Unternehmen und seine Gründer: Im Gegensatz zu der ersten Option, handeln die Erzähltextinhalte in diesem Ansatz von dem Unternehmensgründer. Aufgrund der starken Personalisierung leben diese Erzähltexte von der persönlichen Geschichte des Gründers, mit allen Unwägbarkeiten, die er bewältigte.

3. Das Unternehmen im historischen Kontext: In diesem Ansatz geht es darum, das Unternehmen in den zeitlichen Kontext einzubinden. Wirtschaftliche und gesellschaftliche Hintergründe werden im Zusammenhang mit bezeichnenden Innovationen geschildert und es wird gezeigt, inwiefern diese Wegbereiter für bestimmte (gesellschaftsrelevante) Entwicklungen waren.

Schach verweist darauf, dass ein Erzähltext durchaus in verschiedene Teilthemen untergliedert sein kann, die in verschiedenen Textsegmenten hierarchisiert werden. Daher sind auch Mischformen aus den drei Ansätzen gegeben. In den hier 
untersuchten Daten spiegelt sich verstärkt der erste Ansatz in den Gründungsgeschichten von Edeka und Rewe wider. Da es sich bei diesen Unternehmen um Verbünde von Einzelhandelsunternehmen handelt, ist das eine naheliegende erzählerische Strategie. Ähnlich verhält es sich in der Unternehmensgeschichte von E.ON oder der EnBW, da auch diese Unternehmen aus Fusionen kleinerer Konzerne hervorgegangen sind. Die zweite Entwicklung des Textthemas findet sich in der Gründererzählung über Werner v. Siemens wieder, während die dritte Option sich in den Erzähltextinhalten von Daimler, Volkswagen und Bosch widerspiegelt. Auch die Dt. Telekom bettet ihre Unternehmensgeschichte in den historischen Kontext ein, indem sie die Erfindung des Telefons und des Internets aufrollt, um sich darin zu verorten. Von Telefonica Dtl. liegt keine Unternehmenserzählung vor. Während in dem Korpus von Schach die Varianten zwei und drei am häufigsten vorkamen, sind es in den vorliegenden Daten die Varianten eins und drei. Auch wenn man nun argumentieren kann, dass die Mehrzahl der untersuchten Unternehmen keine spezielle Gründerpersönlichkeit anführen können, hätten sie durchaus die Möglichkeit, eine andere charismatische oder einflussreiche Person für ihre uRE ins Feld zu führen.

\subsubsection{Text-Bild Relationen}

Das Internet bietet Unternehmen die Möglichkeit, ihre Gründungserzählung auf verschiedene Art und Weise umzusetzen. Die einfachste Darstellung entspricht im Grunde einer printmedialen Realisierung, in der Form eines E-Textes. Die Gestaltung von E-Texten entspricht der einer Broschüre im PDF-Format. Häufig bieten Unternehmen wie bspw. Volkswagen oder Rewe Interessierten die Option an, sich die auf der U.-Homepage häufig animierte Geschichte als PDF herunterzuladen. Die zweite Möglichkeit besteht in der Kodierung mittels Hypertext. Nach Storrer (2008: 318) bezeichnet ,Hypertext ${ }^{\star}$ Texte, die durch Computertechnik verwaltet werden und nicht-linear (non-sequential) angelegt sind. In diesen Fällen sind die Erzähltexte über mehrere Module verteilt. Dabei kann jedes Modul weitere Verlinkungen beinhalten, die es den Nutzer/innen ermöglichen, sie nach ihren Interessen (bzgl. Inhalt und Reihenfolge) selbstbestimmt zu rezipieren. Dabei ist für diese Variante charakteristisch, dass sie multimodal, also in Form von Text, Bild, Ton oder Video umgesetzt ist. Realisiert wird diese Option z. B. bei Bosch, Rewe oder Volkswagen anhand einer Chronik mit einer Zeitachse, die in kleine Textabsätze unterteilt ist (vgl. Schach 2016: 88), wie in der untenstehenden Abbildung (Abb. 9.1): 


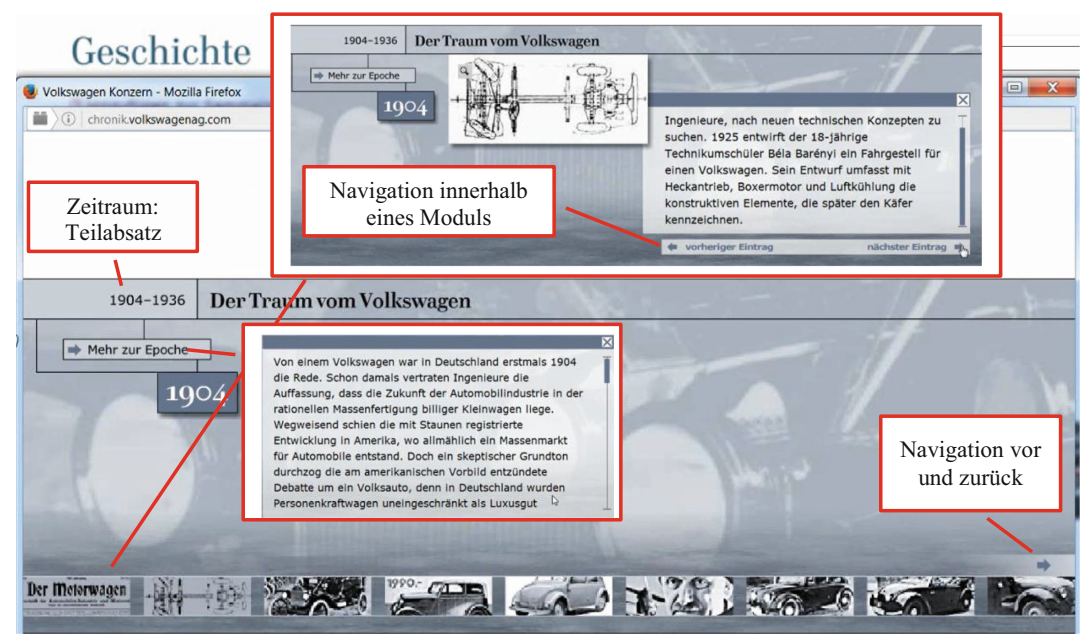

Abb.9.1 Unternehmensgeschichte von Volkswagen anhand eines Zeitstrahls mit Hypertextkodierung (U.-Homepage Volkswagen, o. J. Korpusquelle: VW_396_WS)

Chroniken können entweder als (a) reiner Text, (b) als Text-Bild-Format oder (c) als Flash-Animation auftreten. Schach beschreibt sie folgendermaßen (2016: 93 f.):

a) Chronik als reiner Text: Der Text ist nicht modular aufgebaut und basiert ausschließlich auf Jahreszahlen, die kurze Informationen mit unternehmensrelevanten Ereignissen ergänzen. Dabei handelt es sich um eine stichpunktartige Auflistung in kompakten Sätzen. Im Folgenden zur Veranschaulichung ein Auszug von E.ON (Korpusquelle: E.ON_623_WS): 


\section{e.on}

$2000-2016$

2016

April

E.ON startet mit dem Verkauf seines Stromspeichers in Deutschland

E.ON beginnt mit dem Verkauf seines Stromspeichers E.ON Aura in Deutschland. Die ganzheitliche Energielösung umfasst neben dem Speicher auch die E.ON Aura App sowie auf Wunsch E.ON Aura Strom und eine E.ON Aura Photovoltaikanlage. Für die Entwicklung des Speichersystems arbeitet der deutsche Energieanbieter eng mit der SOLARWATT GmbH aus Dresden zusammen.

Mit seinem Wirkungsgrad von 93 Prozent gehört E.ON Aura zu den effizientesten Stromspeichern auf dem deutschen Markt. Die Unabhängigkeitsquote, das heißt der Anteil des Stroms, der selbst zuhause erzeugt und verbraucht wird, kann damit von etwa einem Drittel auf rund 70 Prozent gesteigert werden. Mit einer Kapazität von 4,4 Kilowattstunden kann E.ON Aura den kompletten Strombedarf einer drei bis vier-köpfigen Familie für die Abend- und Nachtstunden decken. Damit ist er optimal auf den Energieverbrauch eines durchschnittlichen deutschen Privathaushalts ausgerichtet.

März

Ein Drittel aller EEG-Anlagen speisten 2015 in E.ON Netze ein

Bis Ende 2015 waren in Deutschland 34 Prozent aller Erzeugungsanlagen, die nach dem Erneuerbare Energien Gesetz (EEG)

b) Chronik mit Text und Bild: Sind Text und Bild miteinander verknüpft und bilden so eine Einheit, stellen sie einen multimodalen Gesamttext (vgl. „Sprach-Bild-Text“, Sandig 2006: 455) dar. Die Rezipient/innen können die zeitliche Abfolge der Elemente selbst bestimmen, da der Gesamttext in charakteristischer Weise für Hypertexte modular aufgebaut ist (siehe das Bsp. von VW oben). Dabei können ebenfalls Videos statt Einzelbildern eingebunden sein.

c) Chronik als Flash-Animation: „Der multimodale Gesamttext wirkt zunächst über die animierten Fotos oder Bildelemente, erst im zweiten Schritt über die Texte an sich" (Schach 2016: 94). In dieser Variante ist die bewegte Inszenierung dominant. „Grundlage der Animation ist meist ein Zeitstrahl, der die Entwicklung des Unternehmens und die wichtigsten Stationen in einer durch den Nutzer steuerbaren Animation darstellt." (ebd.) Klickt ein/e Nutzer/in auf Bilder oder Jahreszahlen im Zeitstrahl, öffnet sich ein Dialogfenster, in dem ein Text über den Zeitraum informiert (vgl. Schach 2016: 94). Zusätzlich fügt bspw. Rewe spielerische Elemente ein. Hier können die Rezipient/innen mittels des Schieberegler-Symbols weitere Informationen einholen. In der folgenden Abbildung ist es die Veränderung des Rewe-Logos im Zeitraum von 1932-1935 (Abb. 9.2): 


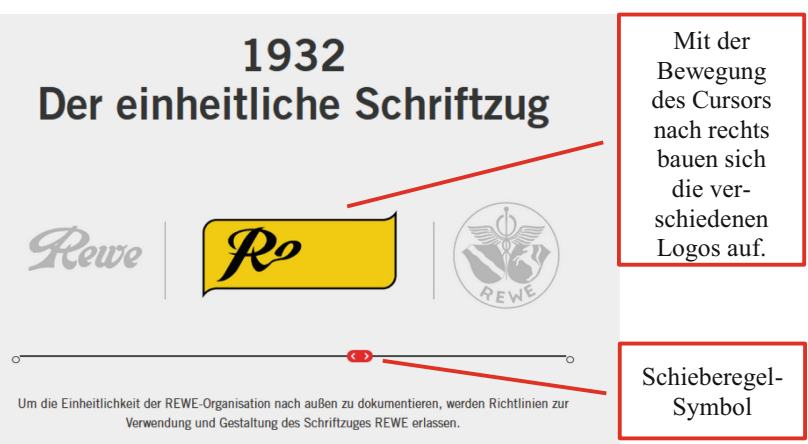

Abb.9.2 Spielerische Animationselemente in der Gründungsgeschichte von Rewe (Rewe, U.-Homepage, o. J. Korpusquelle: REWE_313_WS)

Eine rein textuell realisierte Gründungserzählung findet sich in den vorliegenden Daten nur im Fall von E.ON. Große Unternehmen wie Siemens und Bosch leisten sich für die Präsentation ihrer Geschichte gleich mehrere Realisierungen. Am häufigsten tritt die Chronik mit Text und Bild in dem Korpus auf. Das betrifft die Daten der Unternehmen EnBW, Dt. Telekom, Bosch, VW, Daimler, Siemens und Rewe. Während EnBW, Daimler, Siemens und Dt. Telekom ihre Historie im PDF-Format präsentieren, setzen Bosch und VW das Format modular, anhand eines Zeitstrahls um. Die Repräsentation enthält neben dem Zeitstrahl interaktive Elemente und rudimentäre Bewegtbilder. Daher fasse ich die Chronik ebenfalls unter der Text-Bild-Realisierung. Eine Flash-Animation ist sehr aufwendig. Aus diesem Grunde ist sie vergleichsweise selten und wird zum Erhebungszeitpunkt von den Unternehmen Edeka und Siemens genutzt.

Die Text-Bild-Zusammenhänge erläutert Schach (2016: 95) anhand von drei Beziehungstypen nach Nöth (2000):

1. Redundanz: Das Bild ist textergänzend, demnach liegt eine zweifache Kodierung vor, durch die die Informationen intensiviert werden oder der Text dekoriert.

2. Dominanz: Das Bild ist überwertig und daher informativer als der Text.

3. Komplementarität: Die Rezipient/innen benötigt beide Informationsquellen um den Gesamttext vollkommen zu erfassen.

Wie auch in den vorliegenden Daten sind die Bilder in Schachs Untersuchung in der Regel redundant, da sie „spiegelbildlich zur Information des Textes [stehen]“ 
(Schach 2015: 95). Letztlich illustrieren sie die Textinhalte, ohne zusätzliche Informationen von Relevanz für den Erzähltext anzubieten. Typische Bilder sind Produktbilder, Verträge und Patente, Produktionsstätten, Wohngebäude, Landkarten zur Veranschaulichung von Strecken, Portraits u. a. Komplementär ist die Text-Bild-Beziehung z. B. dann, wenn das Bild eine Veränderung in der Zeit (historische Bilder) abbildet, die nicht im Text aufgegriffen wird. Die folgende Abbildung illustriert eine komplementäre Text-Bild-Beziehung in der FlashAnimation von Rewe. Die Überschrift 1944 Die Schatten des Krieges steht in Beziehung zu dem animierbaren Foto (mittels Schieberegler-Symbol) darunter, das im bildlich kontrastiven Damals-heute-Vergleich das Ausmaß der Zerstörung in Kriegszeiten visualisiert. Indem die Rezipient/innen die Information des Textes Schatten des Krieges und da die Front immer näher rückt und die zwei Bilder kognitiv zusammenführen, verdeutlichen die Bilder, was andernfalls aufwendiger zu versprachlichen wäre (Abb. 9.3).

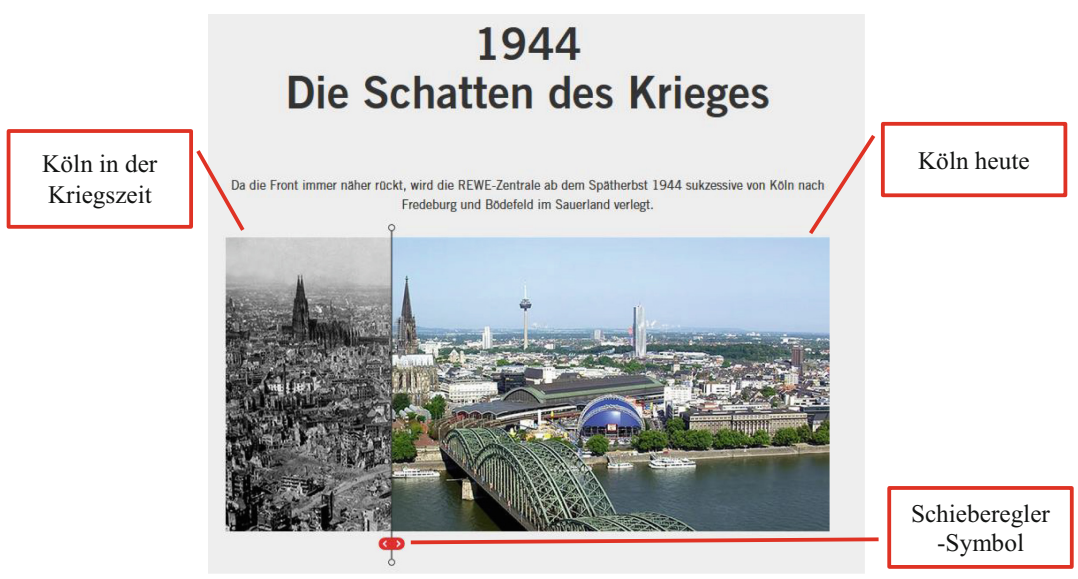

Abb.9.3 Komplementäre Text-Bild-Beziehung in der Unternehmensgeschichte von Rewe (Rewe, U.-Homepage, o. J. Korpusquelle: REWE_313_WS)

Bilder finden sich - mit Ausnahme von E.ON - in allen erhobenen Gründungserzählungen, da gerade Bilder aus der Vergangenheit eine Belegfunktion für die Erzähltextinhalte erfüllen sollen. Eine deutliche Häufung von Bildern findet sich in der Version Chronik als Flash-Animation. Bewegtbilder sind in meinen Daten marginal vertreten. Es wurde eine Gründererzählung von Siemens erhoben, die im Ganzen auf einer Animation beruht. Auf diese Realisierungsform geht Schach 
in ihrer Untersuchung nicht ein. Daher widme ich dieser Erzählung am Ende ein eigenes Unterkapitel (Abschn. 9.1.2.2).

\subsubsection{Narrative Typen der Unternehmensgeschichte}

Insbesondere aufgrund textinterner Faktoren (z. B. Protagonist/innen, Texthemen, sprachliche Merkmale, Themenentfaltung u. a.) identifiziert Schach drei unterscheidbare Erzähltypen in ihrem Korpus. Hier steht sie vor einer ähnlichen Problematik, wie sie in der vorliegenden Untersuchung besteht: Die Texte sind nicht in jedem Fall prototypisch für Erzählungen im klassischen Sinn. Die Autorin diskutiert diese Problematik nicht vertiefend aus der narratologischen Perspektive. Sie nimmt die Erzählungen als Unternehmensgeschichten an und bezieht sich auf die zwei narrativen Strukturierungsmuster nach Heinmann/Viehweger (1991: 238 ff.). Demnach zeichnet sich das erste Strukturierungsmuster durch eine sachlich-registrierende Ereignisdarstellung aus, bei der Schach nach Objektivität im Sinne der Überprüfbarkeit sucht. Sie gibt wieder, was sie für den Handlungsablauf als relevant erachtet und fokussiert dabei die Konsequenzen. Heinemann/Viehweger nennen das Muster NARR I mit REFERIERENDERStruktur (ebd. 1991: 239). Kennzeichnend dafür ist, dass die Erzählung auf das Ergebnis abzielt. Im Gegensatz dazu steht NARR II mit ERZ̈̈HL-Struktur (ebd. 1991: 241 f.). Dieses Muster steht für das, was modellhaft als Erzählung gilt. NARR II ist gekennzeichnet von der Erlebnisperspektive der Erzähler/innen, die sie mithilfe subjektiv-wertender Elemente formulieren. Das Muster weist eine Situierung und eine Ereignisrepräsentation auf, die den Kern des Erzähltextes bildet. Sie besteht aus einer Komplikation, die die Erzähler/innen letztlich auflösen und abschließen. Auf der Basis dieser Konzeption erfasst Schach die Gründungsgeschichten als Erzählungen. Ausgehend von den gesamten Ergebnissen ihrer Untersuchung beschreibt sie drei narrative Typen: Gründerstory, Meilensteine des Unternehmenserfolgs und Ergebnisprotokoll der Unternehmensgeschichte. Nach diesen Typen lassen sich die Daten ebenfalls unterscheiden. Inwiefern es sich hierbei aus der narratologischen Perspektive überhaupt um Erzählungen handelt, reflektiere ich später. Zuerst beschreibe ich die drei Typen, die Schach erhoben hat, da sie den ,narrativen“ Typen von Gründungserzählungen entsprechen:

1. Gründerstory: In der Geschichte steht der Gründer im Mittelpunkt. Er ist der Held, der sich gegen Widrigkeiten behauptet und Herausforderungen annimmt. Er wird als Visionär charkaterisiert, der sich innovativ und entscheidungsfreudig verhält. Persönliche Informationen über den Gründer sind mit der beruflichen Vergangenheit verknüpft und werden auf das heute bestehende Unternehmen übertragen. Die Geschichte entspricht dem ereignisorientierten 
Strukturmuster NARR II. Anschauliche Darstellungen, aktiver Satzbau und „semantische Aufwertung in argumentativen Passagen“ (Schach 2016: 141) zeichnen die Erzähltexte aus.

2. Meilensteine des Unternehmens: Die Akteure in dieser Struktur sind die Unternehmen. Anhand der Meilensteine erscheint die Unternehmensgeschichte wie eine Erfolgsbilanz. Bewertungen erfolgen hier implizit und anhand semantischer Aufwertungen durch die Wortwahl und durch Phraseme. Die Sprache ist tendenziell konzeptionell schriftlich angelegt. Die Tonalität ist werblich vor einem imagebildenden Deutungsrahmen. Bezeichnend ist, dass sich kleine narrative Sequenzen mit einer argumentativen und ergebnisorientierten Vertextung abwechseln (Schach 2016: 146). Inhaltlich kann entweder ,das Unternehmen und seine historische Errungenschaft im Vordergrund stehen" (Schach 2016: 142) oder eine wichtige Erfindung, und/oder es werden historische Verläufe mit dem Unternehmensimage verwoben. Das Strukturmuster entspricht dem, das Heinemann/Viehweger (1991) als NARR I beschreiben.

3. Ergebnisprotokoll der Unternehmensgeschichte: Hier steht vorrangig die Information im Vordergrund. Daher ist der Text von einem neutralen Stil gekennzeichnet in Form einer Darstellung, ,die wesentliche Aspekte der Unternehmensentwicklung protokolliert ohne diese zu bewerten bzw. aufzuwerten" (Schach 2016: 147). Da die Akteure fehlen, überwiegen in den Texten Passivkonstruktionen (z. B. Nominalkonstruktionen). Hin und wieder können kleine „Anekdoten“ in die vorwiegend sachliche Struktur eingebunden werden. Auch hier setzt Schach das Strukturmuster NARR I an.

Die drei narrativen Typen von Schach offenbaren ein bezeichnendes Phänomen mit Blick auf das Erzählen im Unternehmenskontext: Was eine Geschichte ausmacht, was eigentlich narrativ ist, tritt in den Hintergrund. Das Etikett ,Geschichte" als Anreiz für die Rezipient/innen wird in diesem Zusammenhang unreflektiert vergeben. Aus diesem Grund gehe ich im Folgenden auf diesen Aspekt anhand von zwei grundlegend unterschiedlichen Beispielen aus der narratologischen Perspektive ein.

\subsubsection{Reflexion zweier „Extrembeispiele“}

Schach (2016) stellt in ihren Daten fest, dass die Erzähltexte zur Unternehmensgeschichte in hohem Maß von der Informationsvermittlung geprägt sind. Hinzu kommt eine Appellfunktion. Im Rahmen der Unternehmenskommunikation „orientieren sich [informative Texte] an tatsachenbetonten Pressetextsorten“ 
(ebd. 2016: 126), während Texte mit Appellfunktion typisch für Werbetexte sind (Aufforderungshandlung). Gerade für imageprägende Texte mit Wirklichkeitsbezug, deren Gelingensgrundlage ,Glaubwürdigkeit ' ist, ist ein werblicher Erzählstil kontraproduktiv. Daher steht die Informationsfunktion mit einer Fokussierung auf Daten und Fakten im Vordergrund. Dass Unternehmen sehr unterschiedliche Wege gehen, um diese Informationen in Form einer Geschichte zu transportieren, habe ich zuvor in Übereinstimmung mit Schachs (2016) Ergebnissen ausgeführt. Die Analyseergebnisse stellt die Autorin anhand prototypischer Beispiele dar. In den nun folgenden Kapiteln greife ich aus den vorliegenden Daten zwei Beispiele heraus, die zwar an Schachs Ergebnisse anknüpfen, aber in ihren Ausführungen entweder aus der narratologischen Sicht nicht vertieft oder nicht behandelt werden. Das erste Beispiel stammt von Rewe und stellt genau das Gegenteil dessen dar, was gemeinhin als Erzählung gilt. In diesem Fall geht es um eine Umsetzung der Unternehmensgeschichte, die die Frage danach aufwirft, ob dieses Vermittlungsvorkommen aus narratologischer Sicht überhaupt als Erzählung zu fassen ist. Dadurch wird die Kluft deutlich, die zwischen dem Anwendungskontext und einer narratologischen Betrachtungsweise besteht. Das zweite Beispiel ist eine animierte Gründererzählung über Werner von Siemens, die das Unternehmen anlässlich des 200-jährigen Gründergeburtstages auf die unternehmenseigene History-Website stellte. Drängt sich hinsichtlich der Unternehmensgeschichte von Rewe der Eindruck auf, dass die Ankündigung „Geschichte“ als Buzzword fungiert, erscheint das Erzählerische in der Gründergeschichte von Werner von Siemens als die angestrebte Strategie.

\subsubsection{Eine nicht-erzählte Unternehmensgeschichte: Ergebnisprotokoll}

In dem narrativen Typ ,Ergebnisprotokoll“ steht laut Schach (2016: 148) die Informationsweitergabe im Vordergrund. In den von mir untersuchten Daten stellt sich teilweise die Frage, inwiefern die als Unternehmensgeschichte deklarierte Darstellung überhaupt etwas mit einer Erzählung zu tun hat. Auf eine solche ,nicht-erzählte“ Unternehmensgeschichte gehe ich im Folgenden ein. Es geht um die Flash-animierte Unternehmensgeschichte von Rewe. Sie entspricht nach Schach (2016) dem narrativen Typ ,Ergebnisprotokoll`, der mithilfe eines Zeitstrahls und verschiedener interaktiver Inhalte umgesetzt ist. Während die Rezipient/innen auf dem Zeitstrahl die Zeiträume auswählen können, steigern interaktive Inhalte das Rezeptionserlebnis. Zum Einstieg in die einzelnen Kapitel spielt eine fröhliche Blasorchester-Musik. Sobald die/der Rezipient/in in das Kapitel einsteigt, verstummt die Musik. Exemplarisch folgt ein Screenshot des einleitenden Kapitels in die Unternehmensgründung (Abb. 9.4): 


\section{- $\quad$ Zeitstrahl \\ Die Gründung}

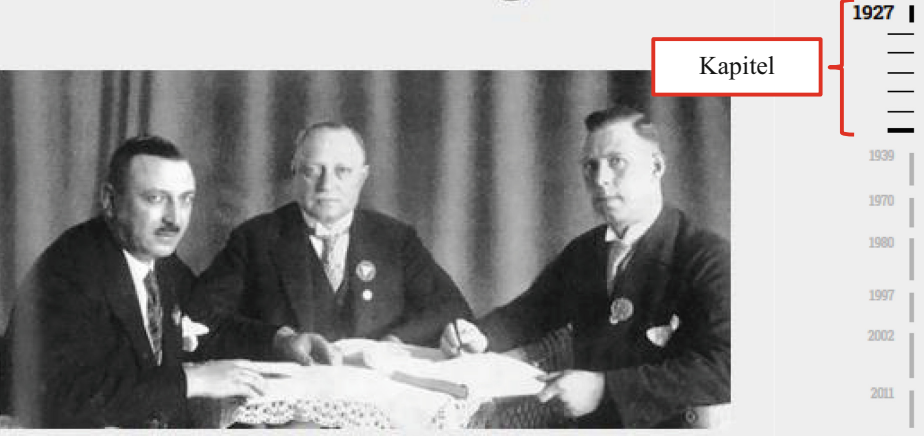

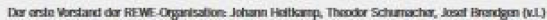

Die REWE-Zentrale nimmt ihre Taligkeit in Koln auf und wird in das Genossenschaltsregister eingelragen. Ende 1926 hatter 17 Einkaulsgenossenschaften die Grandung beschlossen. Der gewahlte Name REWE keitet sich von „Aevisionsvertand der Westkauf-Genossenschaften" ab.

Ziele und Absichten der Genossenschaft werden im Grandungrstahut wie folgt festgelegt: _Gegensland des Untemehmens ist nach dern Grandungsslatut die Forderung des Erwerts und der Wirtschalt ihrer Mitglieder miltels gemeinschaftlichen Geschaftsbetriebs insbesondere durch gemeinschaltlichen Einkauf und Vermittlung von Waren far die Mitglieder."

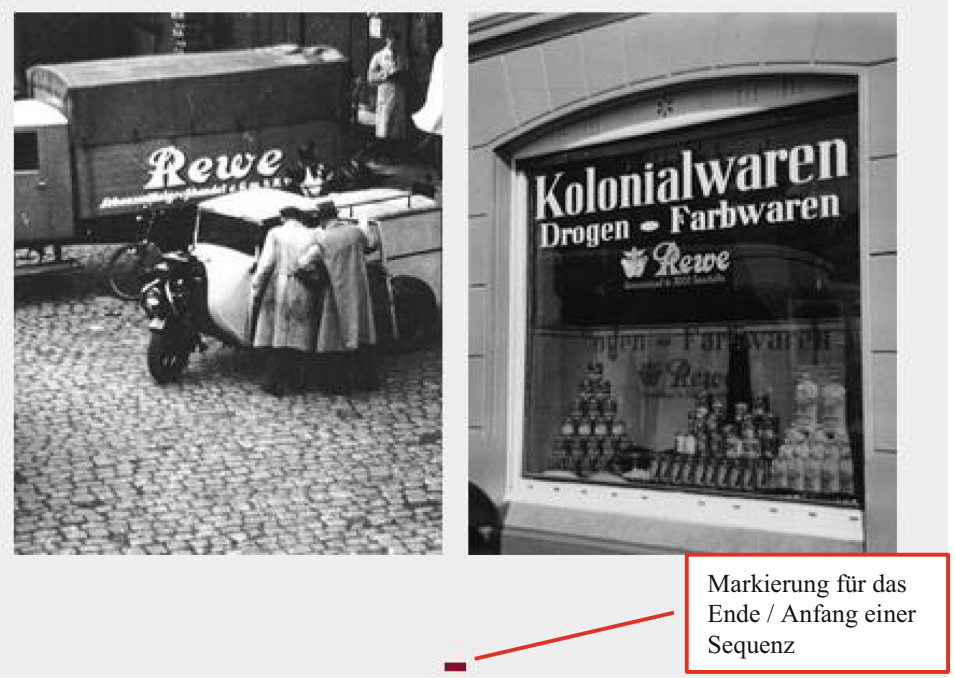

Abb.9.4 Typ ,Ergebnisprotokoll der Unternehmensgeschichte“ von Rewe (U.-Homepage, o. J. Korpusquelle: REWE_313_WS) 


\section{Haupthandelsartikel in 1931}

Drei Jahre nach der Grandung konnte die neue Genossenschaft schon viele Artikel urrsetsen. Hier sind die an hauligsten gehandelten Waren des Jahres 1931 dargestellt.

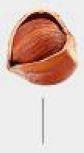

1,000 Kisten Haselnüвse

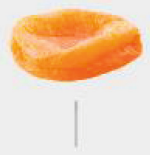

1.500 Kisten getrocknete Aprikosen

$=$

\section{2 \\ Der einheitliche Schriftzug}
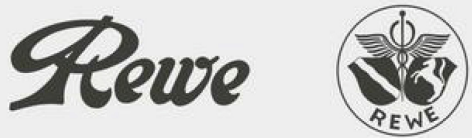

Interaktives

Element: Schieberegler

\section{5 \\ Die erste Expansion des Wirtschaftsraums}

Durch die Autnahme von Sodkaut Neustadt sowie des Stettiner und Dresdener Bezirksrentralen verlagert sich Mitte der $30 \mathrm{er}$-Jahre das wirlschaltliche

Zentrum der REWE-Zentrale vom ineinischen Wirtschaftsraum in Richtung Mitteldeutschland.

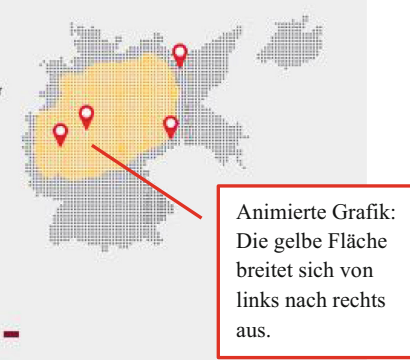

Abb.9.4 (Fortsetzung) 


\section{Vorstandsvorsitz}

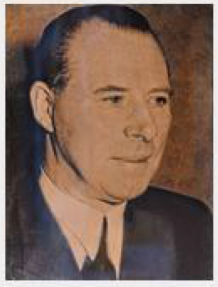

Josas Anandyen

(1927-199:2)

$=$

Impressionen

1927 - 1938

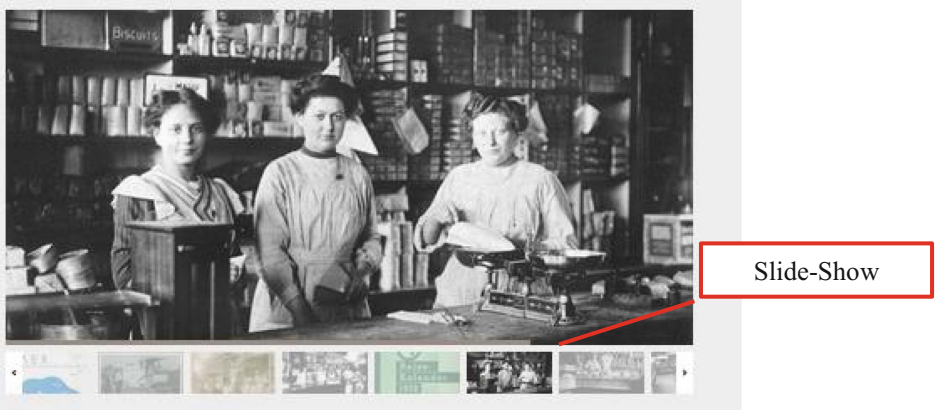

Abb. 9.4 (Fortsetzung)

Den Kapitelverlauf strukturiert die chronologische Abfolge der Jahreszahlen von oben nach unten. Text, Bilder, animierte Grafik, eine abschießende Slide-Show und ein interaktives Element sind die Gestaltungselemente. Die Überschrift kündigt an, was in der Sequenz darunter folgt. Die Bilder sind zum Teil redundant, d. h. dekorativ (abgebildete Haselnuss usw.), oder redundant 
mit intensivierender Wirkung (gelbe Grafik von der Ausbreitung der Edekamärkte Richtung Mitteldeutschland), dominant (Foto des Vorstandsvorsitzenden) und komplementär im Falle des interaktiv veränderbaren Rewe Schriftzugs. Im Sinne der uRE handelt es sich funktional überwiegend um Zier- oder Schaubilder, die die Geschichte selbst nicht vorantreiben. So zeigt bspw. die Slide-Show unzusammenhängend aneinandergereihte Fotografien. Im Begleittext tritt das Unternehmen bzw. seine Teilunternehmen als Akteur auf. Fotografisch dargestellte menschliche Akteure, wie Vorstände oder in der Slide-Show abgebildete ehemalige Mitarbeiter/innen, kommentiert der Text nicht. Insgesamt ist in der Flash-Animation wenig Text vorhanden. Inhaltlich wird darin auf Daten und Fakten Bezug genommen, die ein Ergebnis anzeigen (z. B. Haupthandelsartikel, einheitlicher Schriftzug, Expansion). Die Syntagmen sind weitgehend daran ausgerichtet Maßnahmen zu begründen (z. B. Um die Einheitlichkeit der Rewe [...], Durch die Aufnahme [...], Ziele und Absichten der Genossenschaft [...]). Diesbezüglich ist schon hier fraglich, inwiefern überhaupt von einer Geschehensdarstellung die Rede sein kann ${ }^{5}$. Temporale Gliederungshinweise (Jahreszahlen und Zeitangaben: Drei Jahre nach der Gründung) sowie begründende Satzeinleitungen ( $\boldsymbol{u m}$... z $\boldsymbol{u}$, durch ...) vermitteln Zusammenhänge zwischen den einzelnen Abschnitten. In den Textelementen überwiegt die Präsensverwendung sowie ein sachlich-neutraler Stil. Der Stil entsteht durch Passiv- und Infinitivkonstruktionen, aber vor allem durch das Vermeiden von charakterisierenden Adjektiven und anderen Möglichkeiten der semantischen Aufwertung (z. B. Hochwertwörter, Emotionswortschatz u. a.). (vgl. Schach 2016: 147 ff.)

Vor einem narratologischen Hintergrund beginnt die Diskussion über einen narrativen Anspruch bereits in Bezug auf die im Verortungsschema angesetzte Minimalbedingung nach Martinez (2017), mit Blick auf die Geschehensdarstellung (vgl. Abschn. 5.1). Zutreffend ist, dass konkrete, singuläre Sachverhalte gegeben sind (Unternehmensgründung, erster erfolgreicher Handel, symbolisch markierter Zusammenschluss der Einzelhändler anhand eines gemeinsamen Logos, Expansion). Daher ist das Merkmal der Konkretheit erfüllt. Auch die Temporalität entlang eines Zeitverlaufes ist gegeben, in dem auf das Unternehmen Bezug genommen wird. Die Kontiguität zwischen den Ereignissen und der Geschehensdarstellung ist vage in der zeitlich-räumlichen Verbundenheit auszumachen, denn betrachtet man die Aussagen näher, stehen die Abschnitte bzw. darin benannten Ergebnisse sprachlich in keinem kausalen Zusammenhang. Das bedeutet, an diesem Element der Minimalbedingung steht die Narrativität der

\footnotetext{
${ }^{5}$ Kindt (1997: 12) führt in seinen Definitionskriterien für eine Erzählung an, dass sie prototypisch überwiegend aus der Darstellung eines Geschehens besteht. Ein Geschehen enthält hierbei Prozessanteile, die bei einer schlichten Ergebnisbenennung unerwähnt bleiben (vgl. ebd. 1997: 13).
} 
Realisierungsvariante erstmals in Frage. Möchte man dennoch die Bedingungen durch implizite kausale Bezüge als erfüllt werten, gilt es nach dem Verortungsschema herauszufiltern, inwiefern die Unternehmensgeschichte innerhalb des Erzähltextes als erzählenswert markiert ist.

Merkmale, die einen Erzähltext als erzählenswert ausweisen, habe ich für das Korpus in inhaltliche und formale unterschieden. Im Hinblick auf inhaltliche Kriterien kann zur Prüfung ausschließlich das Merkmale der Zustandsveränderung nach Schmid (2014) herangezogen werden. Die Evaluation (Labov/Waletzky 1973), das sozial relevant außergewöhnliche Ereignis (Kindt 1997) und die Erfahrungshaftigkeit (Fludernik 1996) sind bezüglich der Geschehensdarstellung an eine vermittelnde Instanz gebunden, während die Zustandsveränderung sich sowohl auf die Akteure als auch auf die erzählte Welt bezieht. Hierfür stellt Schmid drei Bedingungen an die Zustandsveränderung (vgl. Abschn. 5.2.2). Die zweite Bedingung erfordert eine Äquivalenz von Ausgangs- und Endzustand, in Form von Similarität und Kontrast. Das Merkmal Similarität ist darin gegeben, dass die uRE sich um Unternehmensbelange dreht. Der Kontrast von Anfangsund Endzustand bedeutet eine Veränderung. Auch dieses Kriterium stellt die vom Unternehmen versprochene Narrativität in Frage. Eine erkennbare Veränderung ergibt sich in der Darstellung allenfalls über die Entwicklung in der Zeit, z. B. von einem kleinen zu einem großen Unternehmen. Dies ließe sich als die Folge (Konsequenz) aus bestimmten Handlungen/Ereignissen versprachlichen. Das ist in der vorliegenden Unternehmensgeschichte nicht der Fall. Es werden Handlungen/Ereignisse aufgezählt, aber die Konsequenzen wenn überhaupt nur marginal herausgestellt (z. B. Drei Jahre nach der Gründung konnte die neue Genossenschaft [...]). Diese Argumente disqualifizieren die Unternehmensgeschichte ebenfalls für die narrative Vertextungsstruktur NARR I von Heinemann/Viehweger (1991), die Schach (2016) zugrunde legt.

In Anbetracht der im Verortungsschema angesetzten formalen Merkmale schließt bereits der sachlich-neutrale Stil subjektivierende Merkmale - wie Emotionalität, Evozieren von Atmosphäre oder gar Gedankeneinblicke - aus. Bezüglich textstruktureller Merkmale lassen sich nur vereinzelt temporale Gliederungselemente ausmachen, überwiegend stehen die Ergebnisaufzählungen als Textabschnitte lediglich chronologisch geordnet hintereinander. Ausgehend von dem für die Daten entwickelten Verortungsschema besteht die vorgestellte Unternehmensgeschichte von Rewe nicht als Erzähltext. Es zeigt sich, dass in der Gründungserzählung nicht nur narrative Gestaltungsmöglichkeiten zu Gunsten eines Informationsbedürfnisses unzureichend ausgeschöpft werden (wie das Schach konstatiert), sondern unter der Bezeichnung schlicht keine Erzählungen mit Geschehensdarstellungen vorkommen. Die eigentliche Frage ist m. E. demnach nicht, ob eine Erzählung vorliegt, sondern vielmehr, inwiefern Unternehmen deklarierte Geschichten nicht Erzählen. Schach versucht die Beobachtung zu entschärfen, indem sie für ihre Daten von vereinzelt narrativen Einschüben spricht. 
In dem vorgeführten Beispiel sind allerdings grundlegende Voraussetzungen für einen Erzähltext nicht gegeben, sodass wenige narrative Einschübe noch nicht die Kategorisierung als ,Unternehmensgeschichte" rechtfertigen: Informationsvermittlung und spielerische Unterhaltung stehen über der narrativen Inszenierung. Im Kontrast zu diesem Fall folgt nun ein Best-Practice-Beispiel von dem Elektrotechnologie Unternehmen Siemens, konzipiert und durchgeführt von dem Siemens Historical Institute.

\subsubsection{Ein Best-Practice-Beispiel: animierte Gründererzählung}

Zum 200-jährigen Geburtstag von Werner von Siemens ließ das Unternehmen eine Gründererzählung erstellen, die während Schachs Datenerhebung vermutlich noch nicht veröffentlicht war. Diese Erzählung sticht aus den zuvor beschriebenen Daten aufgrund ihrer speziellen Gestaltungsmittel hervor, weswegen ich sie in diesem Kapitel gesondert aufgreife. Grundsätzlich ist die Gründererzählung der narrative Typ, der im Vergleich zu den anderen Typen über eine eindeutig narrative Ereignisdarstellung verfügt, da neben Ergebnissen auch biografische Informationen über die Gründer wiedergegeben werden. (vgl. Schach 2016: 136 ff.) Die Gründererzählung, um die es im Folgenden geht, ist in ihrer Repräsentation speziell: Zum einen vermittelt sie ein Ich-Erzähler durch voiceover, der sich als Werner von Siemens vorstellt und als Figur in der Animation auftritt: allerdings nicht in der Funktion des Erzählers, sondern ausschließlich zur Geschehensdarstellung. Zum anderen handelt es sich um eine multimodale Umsetzung in Form einer aufwendigen Flash-Animation, die in Ansätzen an einen Trickfilm erinnert. Bevor ich auf die zwei genannten Realisierungsoptionen eingehe, stelle ich die narrative Strukturierung der Geschichte anhand der Kapitelinhalte vor, um die darstellungsrelevante Programmatik zu verdeutlichen.

Die narrative Struktur ist in acht Abschnitte/Kapitel gegliedert, die jeweils einen zeitlichen Umfang von zwei bis vier Minuten aufweisen. Die Kapitel können zwar unabhängig voneinander rezipiert werden, da sie jeweils neue Ereignisse wiedergeben, steigt ein/e Rezipient/in jedoch quer ein, versteht er/sie das Kapitel nur mithilfe der Kontextinformation: Gründergeschichte von Werner von Siemens. Insgesamt handelt es sich um eine geschlossene Erzählserie, in der vornehmlich das erste Kapitel mit einleitenden Informationen angereichert ist. Aufgrund genannter Jahreszahlen und der inhaltlichen Entwicklung ist die Handlungsfolge chronologisch organisiert. Das erste Kapitel beginnt damit, dass sich der Erzähler durch voice-over als Werner von Siemens vorstellt. Dabei erscheint eine entsprechende Figur (Standbild 01, siehe linke Spalte im folgenden multimodalen Transkript), die optische Merkmale der realen Person Werner von Siemens 
(Gesichtszüge) aufweist. Zur Verdeutlichung der multimodalen Repräsentation folgt ein multimodaler Transkriptausschnitt aus dem ersten Kapitel:

(Bsp.) Beginn des ersten Kapitels aus der Gründererzählung Werner von Siemens (Siemens, U.-Homepage, 2016. Korpusquelle: SIEMENS_562_WS)

\begin{tabular}{|c|c|c|}
\hline $\begin{array}{l}\mathrm{N} \% \text { min. } \\
\text { Merkmale }\end{array}$ & $\begin{array}{l}\text { Standbild, Kapitel 1, 00:00-00:27 } \\
\text { (Korpusquelle: SIEMENS_562_WS) }\end{array}$ & Ton: Sprachtext \\
\hline $\begin{array}{c}01 / 00: 00- \\
00: 18\end{array}$ & & $\begin{array}{l}\text { Im Hintergrund spielt Klaviermusik: } \\
\begin{array}{l}\rightarrow \text { 01: E: ICH bIn werner von sIEmens; } \\
02: \text { und ich GLAUbe, } \\
\text { 03: dass meine: LEbenserinnerungen, } \\
\text { 04: für junge menschen LEHRreich, } \\
05: \text { und ANspornend sein können. } \\
06: \text { ich will ZEIgen, } \\
07: \text { dass ein junger mann auch oHne } \\
\text { 08: viel geld. } \\
\text { 09: allein durch ARbeit, } \\
\text { 10: und NütZliches tun kann; }\end{array}\end{array}$ \\
\hline $\begin{array}{l}\text { Merkmal: } \\
\text { Das Portrait } \\
\text { wird einge- } \\
\text { zoomt. }\end{array}$ & & \\
\hline $02 / 00: 25$ & & $\begin{aligned} \text { 11: } & \text { und DAS ist meine geschIchte. } \\
\text { 12: } & \text { ich wurde am DREIzehnten dezember, } \\
\text { 13: } & \text { achtzehnhundersechzig in LENdle } \\
& \text { bei hannover geboren, }\end{aligned}$ \\
\hline $\begin{array}{l}\text { Merkmal: } \\
\text { Die } \\
\text { einzelnen } \\
\text { Markierunge } \\
\text { n auf der } \\
\text { Landkarte } \\
\text { erscheinen } \\
\text { nacheinande } \\
\text { r. } \\
\end{array}$ & RMAN & 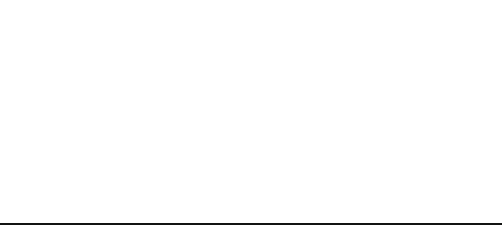 \\
\hline $03 / 00: 27$ & 5 lahre att & $\begin{array}{l}\rightarrow 14: \quad \begin{array}{l}\text { wo ich die ersten acht jahre meines } \\
\text { LEbens verbrachte. }\end{array}\end{array}$ \\
\hline $\begin{array}{l}\text { Merkmal: } \\
\text { Fähnchen } \\
\text { bewegen } \\
\text { sich. }\end{array}$ & 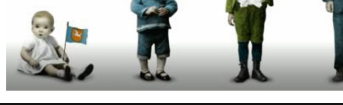 & \\
\hline
\end{tabular}

Der Erzähler stellt sich nicht nur selbst vor, er benennt auch zu Beginn ,seine“ Motivation für die folgende Geschichte, die er mithilfe der Substitution auf MetaEbene und DAS ist meine geschIchte (Z. 11) eröffnet. Im Hintergrund spielt ein Klavierstück. Die Musik fungiert in den folgenden Kapiteln als musikalisches Thema. Sobald ein bemerkenswertes Ereignis oder eine Binnenerzählung 
auftritt, verändert sich dementsprechend die musikalische Klangatmosphäre. Die instrumentelle Untermalung wie auch die Geräuschkulisse bilden Cues (Wyss 2011), sogenannte „Interpretationsanleitungen für Sequenzen oder das gesamte audiovisuelle Narrativ“ (Wyss 2011: 287).

Die einzelnen Kapitel behandeln bestimmte Errungenschaften, die in den historischen Kontext gestellt werden, z. B. Kriegsgeschehen und besondere Anlässe (z. B. KURZ nach der fErtigstellung, / konnte die NACHricht zur wahl des deutschen kAIsers, / aus der frankfurter PAULSkirche nach berlIn, / in WEniger als sIeben stunden übermittelt werden.) (Korpusquelle: SEIMENS_563_WS). Jedes Kapitel endet mit einem Resümee, um es abzuschließen. Die folgende Tabelle verdeutlicht, dass die Kapitelinhalte an einer narrativen Strukturierung von Erzähleröffnung (Kap. 1), Ausgangssituation (Kap. 2), Ereignisrepräsentation mit Wende- und Höhepunkten (Kap. 3, 4, 5, 6) und Resümee (Kap. 7, 8) ausgerichtet sind. Ich verzichte auf die vollständigen Transkripte und fasse in der folgenden Tabelle die Inhalte zusammen, da das ausreicht, um die narrative Entwicklung nachzuvollziehen. Zur Verdeutlichung versehe ich die Kapitel mit Überschriften, die eine Zusammenfassung der Inhalte entlang der narrativen Strukturierung ausdrücken. Im Original sind diese Überschriften nicht vorhanden (Tab. 9.1):

Der Aufbau der Erzählung folgt einem Wandel in der Zeit, der in Kapitel untergliedert sowohl die Entwicklung des Protagonisten als auch des Unternehmens nachzeichnet. Die Kapitelzusammenfassung verdeutlicht, dass die Erzählung nach Angaben zu dem Protagonisten (Kap. 1) und einer Schilderung über den Gründungsbeginn (Kap. 2) in Kapitel drei auf einen Wendepunkt zuläuft, der in Kapitel vier Veränderungen anstößt und die Entwicklung des Unternehmens vorantreibt. In Kapitel fünf scheint der Protagonist weniger berufliche Ziele zu verfolgen und mehr persönlichen Interessen - politischer und gesellschaftlicher Art - nachzugehen. Dank seiner politischen Vernetzung erkennt der Protagonist im Folgekapitel günstige Gelegenheiten und expandiert sein Unternehmen nun wieder weiter. In Kapitel sieben hat das Unternehmen des Protagonisten sich aufgrund seines langjährigen Wirkens so entwickelt, dass Werner von Siemens auch die Trennung von seinem Geschäftspartner nicht mehr als unüberwindbare Kriese erlebt. Er delegiert und bringt sein Unternehmen weiter voran. Das Schicksal belohnt ihn nicht nur mit Erfolgen im Geschäftlichen, sondern auch im Privaten. Er schafft es nun sowohl seine politischen als auch seine unternehmerischen Interessen miteinander in Einklang zu bringen und scheint mitten im Leben zu stehen. Das letzte Kapitel zielt abschließend auf die Bewertung des Werkens und Wirkens Werner von Siemens, um daraus die (S)D für das heute bestehende Unternehmen abzuleiten. Die (S)D erfolgt mithilfe der Übertragung der Errungenschaften in 


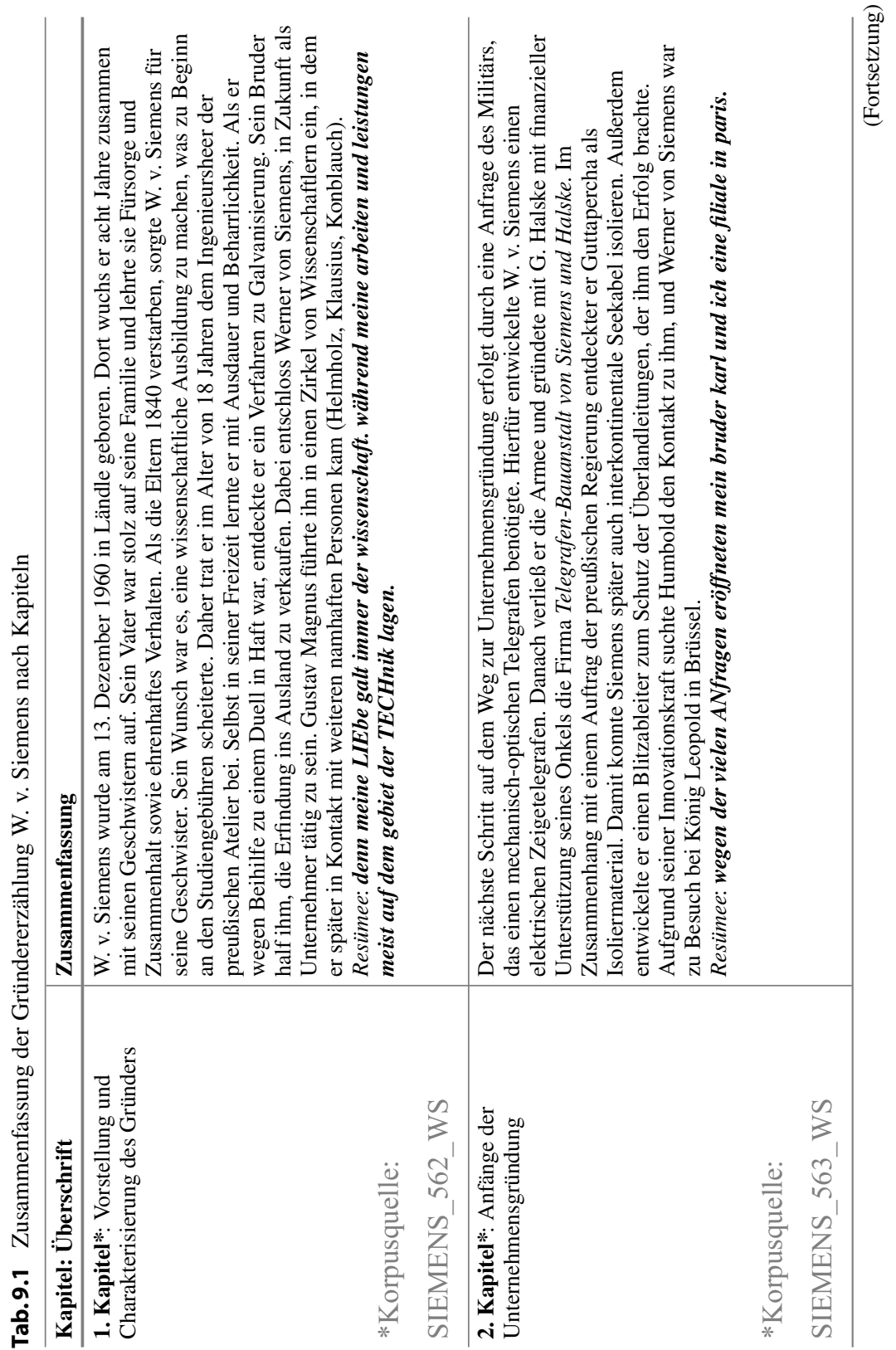




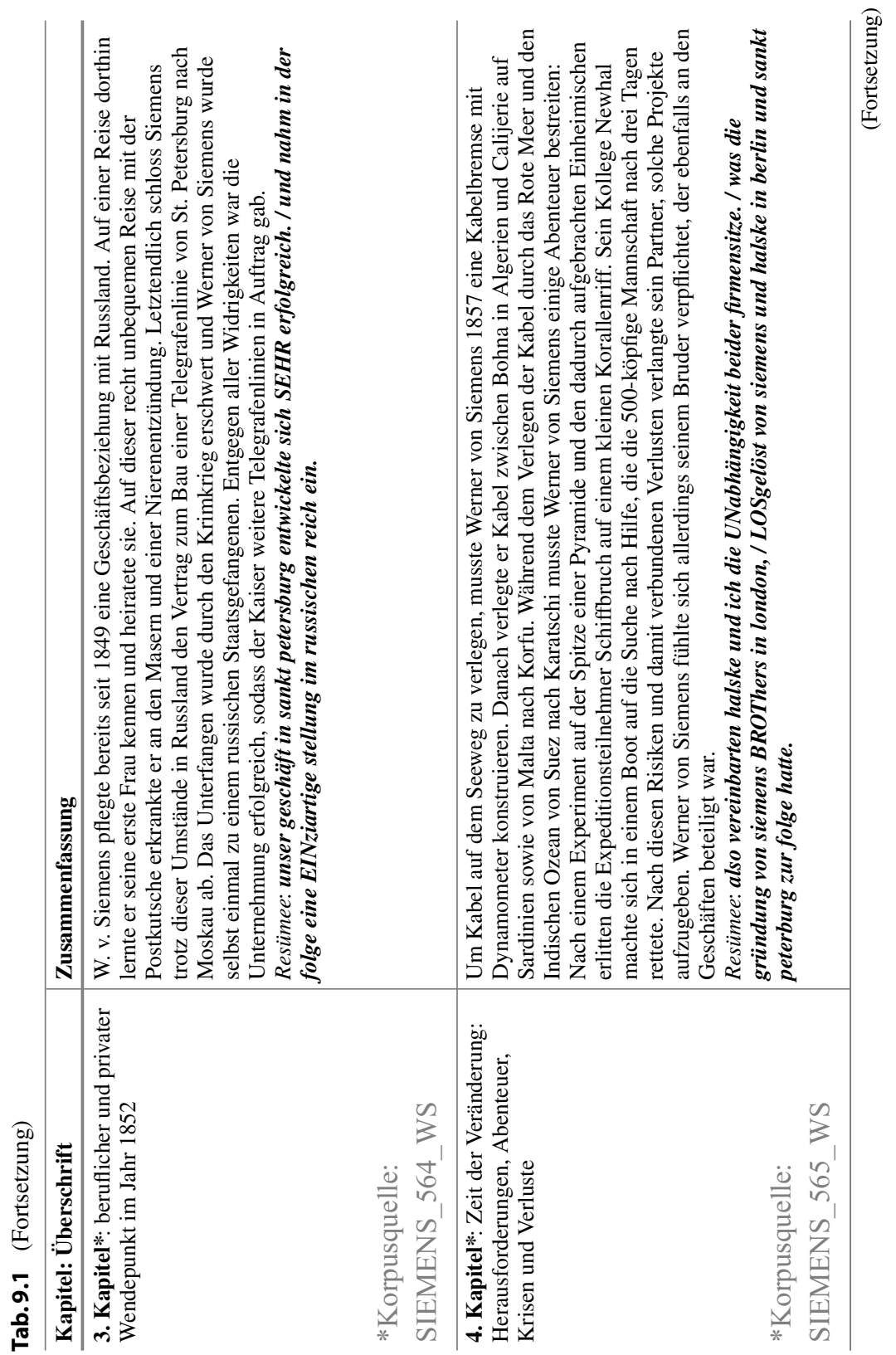




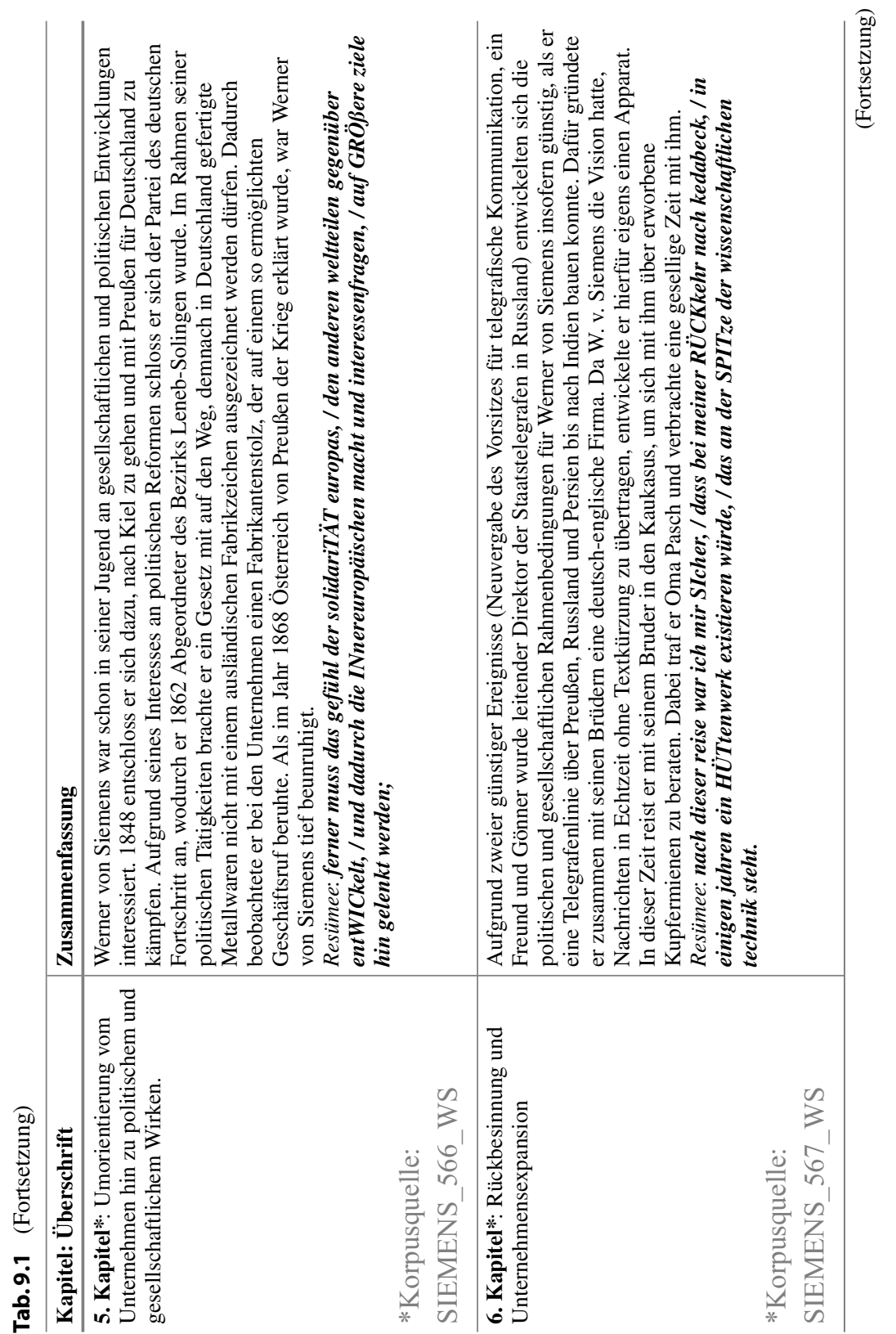




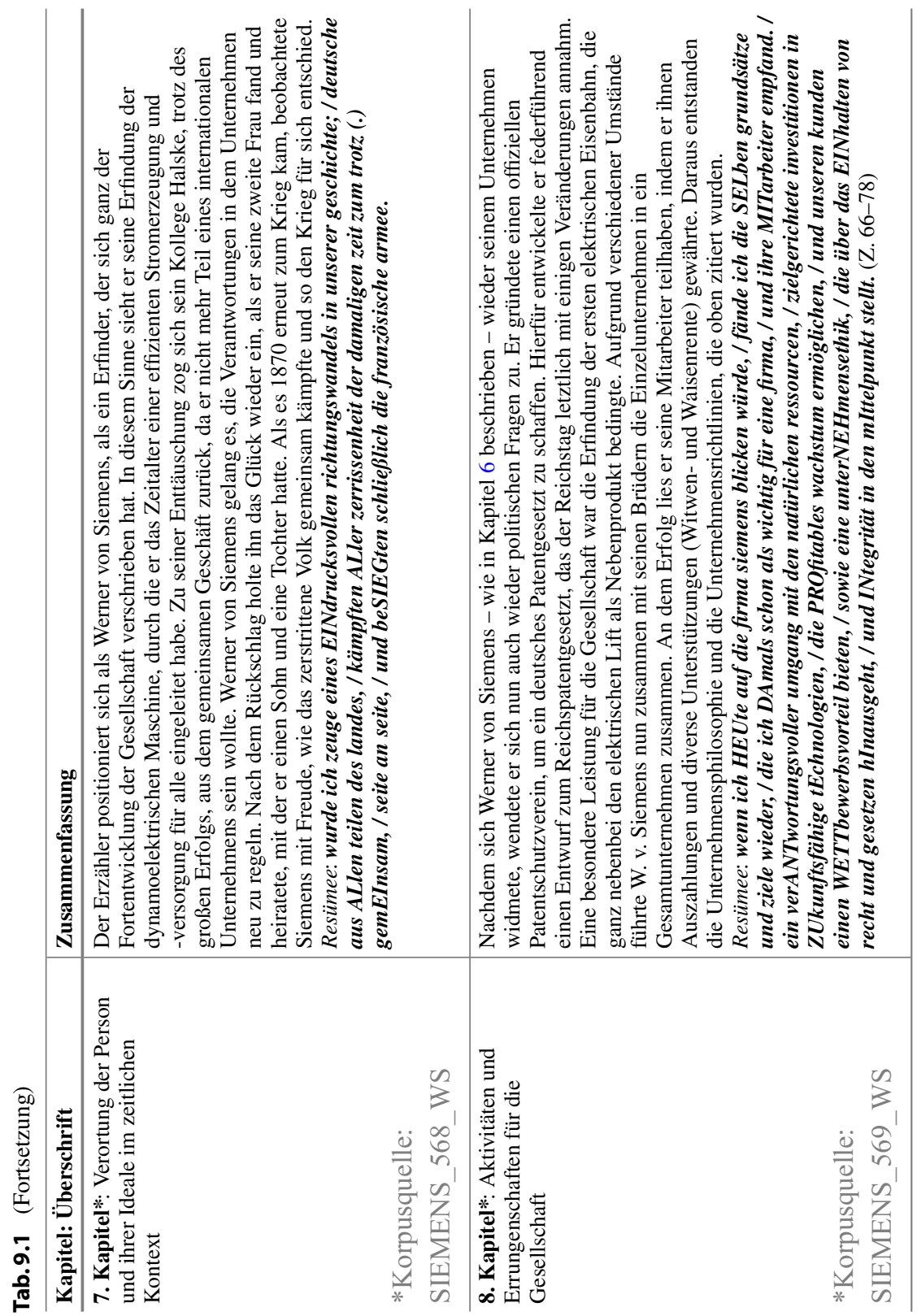


die Jetzt-Zeit. Hierzu leitet der Erzähler aus den zuvor erzählten Handlungen die Unternehmensgrundsätze ab: wenn ich HEUte auf die firma siemens blicken würde, / fände ich die SELben grundsätze und ziele wieder, [...]. Auf diese Weise schließt das Ende an den Anfang an, indem der Erzähler darauf hinweist, dass seine Lebenserinnerung lehrreich sein und als Vorbild dafür dienen sollen, mit viel Arbeit und wenig Geld etwas Nützliches zu erschaffen (Erfolgsgeschichte).

Insgesamt sind die einzelnen Kapitel von argumentativ begründenden und explanativ beschreibenden Passagen durchsetzt, die eine Innovation erklären und ihre Bedeutung oder eine Entscheidungsfindung rechtfertigen, wie die folgenden zwei Beispiele verdeutlichen:

(Bsp.) Explanative und argumentative Passagen in der Gründererzählung von Siemens

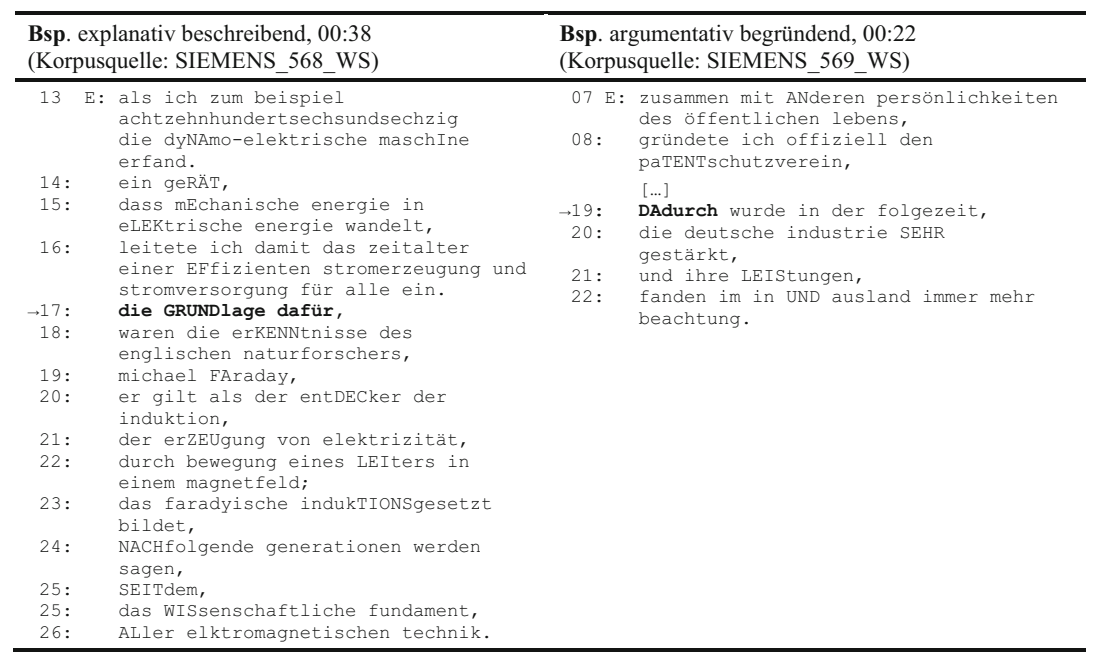

Grundsätzlich werden persönlich bedeutende Ereignisse, wie Heirat, Elternschaft, Todesfall der Eltern oder Inhaftierungen sowie das persönliche Erleben, nicht näher ausgeführt, sondern undramatisch erwähnt. Der Fokus liegt auf den Erfolgen des Gründers, seinen Innovationen, gesellschaftlichen Beiträgen und seinen Verdiensten für das Unternehmen. Auffallend häufig benennt der Erzähler namhafte Personen, die als Interessierte, Verbündete und/oder Unterstützer auftreten und die Bedeutung der Person Werner von Siemens aufwerten.

Zur Umsetzung der Gründergeschichte haben die Autor/innen auf zwei spezifische Repräsentationsoptionen zurückgegriffen. Die erste Option, die ich aufnehme, ist für das vorliegende Korpus ungewöhnlich. Sie beruht auf der 
gewählten Erzählinstanz. Im Zusammenhang mit ihrer Funktion nenne ich die Umsetzung in diesem Kontext das Potenzial des Ich-Erzählers. Es entsteht einerseits durch die große Unmittelbarkeit, die mit einer Ich-Erzählung einhergeht. So weckt eine Ich-Erzählung aufgrund der persönlichen Involviertheit des Erzählers das Rezipienteninteresse und macht die Inhalte besonderes eingängig. Hierfür simuliert das vorliegende Format eine autobiografische Erzählung des Gründers, in der sich ein imaginierter Werner von Siemens Vergangenes als Selbsterlebtes vergegenwärtig. Dabei handelt es sich jedoch um eine Figur. Da die Figur auf einer realen Person beruht und die Erzähltextinhalte einen faktualen Geltungsanspruch auf die außersprachliche Wirklichkeit beanspruchen (Darstellung von Daten und Fakten), steht die uRE in einem Spannungsverhältnis zwischen Fiktion und Faktualität. Das verdeutlichen die Autor/innen visuell anhand des Bewegtbildes, in dem ein cartoonartig animierter Mann mit den Gesichtszügen Werner von Siemens auftritt. Darüber hinaus markieren sprachliche Merkmale die „Illusion“, indem der Erzähler zwar persönliche Informationen einstreut, aber a) nur bedingt emotionale Regungen zeigt und b) aus der erzählten Zeit in die Jetzt-Zeit tritt. Besonders deutlich wird das am Ende des achten Kapitels, als der Ich-Erzähler die zeitliche Erzählperspektive mit dem Temporaladverb damals in die Jetzt-Zeit verschiebt, um die Maßnahme aus der heutigen Sicht zu kommentieren (solche tandJEmen und zUahlungen, / waren damals noch $N E U$, / und UNgewöhnlich, (Korpusquelle: SIEMENS_569_WS) oder in den Konjunktiv Präsens wechselt (heute würde man sagen (Korpusquelle: SIEMENS_565_WS), wenn ich HEUte auf die firma siemens blicken wïrde, (ebd.)).

Andererseits gibt ein auditiv wahrnehmbarer Erzähler nicht nur über die erzählten Inhalte, sondern ebenfalls durch seine sprachliche Performanz etwas über sich preis. Nun handelt es sich in dem vorliegenden Fall nicht um Werner von Siemens. Doch ist mit der Ich-Erzählung verbunden, dass die Autor/innen den Gründer nicht nur durch die Erzähltextinhalte, sondern auch durch sein Erzählen charakterisieren. Auch in diesem Fall markieren die Autor/innen, dass kein natürlicher Erzähler spricht. Beispielsweise ist die Darstellung vergangener Ereignisse konsequent im Präteritum gehalten. Für natürlich mündliche Erzählungen zeigt die linguistische Forschung die Präferenz des Perfekts, während das Präteritum charakteristisch für literarisches Erzählen ist. Ein weiteres Spezifikum ist der gehobene Sprachstil. Er entsteht aufgrund von Phrasen, die veraltet erscheinen: sich zusammen tun, Ansprache halten, etwas beiwohnen, Schiffbruch erleiden, ein Sturm zieht auf. Im Kontrast dazu erscheinen zwischendurch nachgetragene Sätze, die wie eine gesprochensprachliche Ergänzung (Nachtrag/Rechtsherausstellung) wirken sollen: 
(Bsp.) Gesprochensprachliches Merkmal ,Rechtsherausstellung ' in der Gründererzählung von Siemens

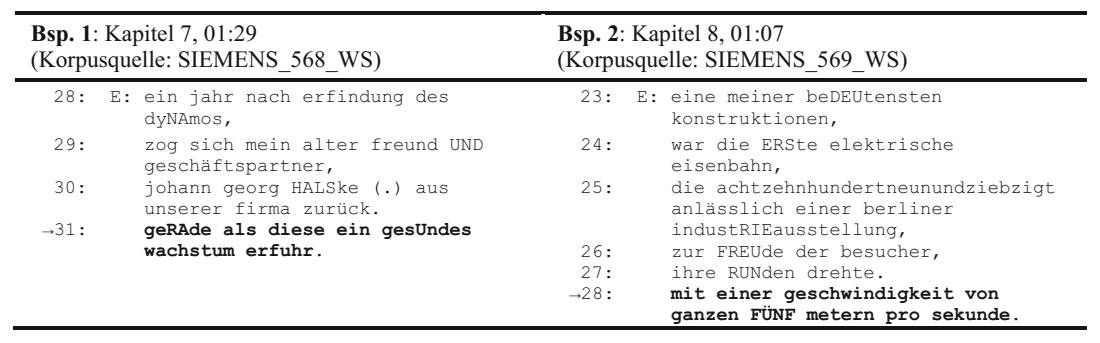

Daneben dehnt der Erzähler vereinzelt leicht die Vokale, die eine gefüllte Sprecherpause andeuten, als müsse er kurz im Redefluss verharren und sich sortieren: u:nd wir drei BRÜder formten nun, / dass meine: Lebenserinnerungen. Durch diese Versprachlichung wird W. v. Siemens als eine Person des gehobenen Bürgertums dargestellt, dem die erzählten Erinnerungen an die Nöte des „kleinen Mannes“ (Existenzsorgen und arbeitsreiche Mühsal) als Kontrapunkt entgegenstehen. Um einen Gründermythos zu stilisieren, inszenieren die Autoren ein kreatives Spiel mit literarisch narrativen Merkmalen und faktualem Erzählen. Beides wird evoziert und stellenweise aufgebrochen: Eine faktuale Erzählung, insbesondere mit autobiografischen Elementen hieße, dass der Erzähler und der Autor übereinstimmen. Das ist in dieser Erzählung nicht gegeben. Eine literarische Erzählung wird wiederum selten gesprochensprachlich von einem Ich-Erzähler realisiert.

Das Potenzial des Ich-Erzählers entwickelt sich (a) dadurch, dass er seine Rezipient/innen durch persönliche Einblicke neugierig macht, auch oder möglicherweise gerade deswegen, weil die Autor/innen deutliche Indizien setzen, um fiktionale Aspekte der Erzählung zu kennzeichnen. (b) Des Weiteren eröffnet ein Ich-Erzähler durch die sprachliche Performanz zusätzliche Möglichkeiten der Personencharakterisierung. Dadurch entsteht eine facettenreiche Figurierung, die nicht nur beschrieben, sondern durch das auditive Format performt wird.

Eine audio-visuell repräsentierte Geschichte im Bewegtbild spricht die Wahrnehmungssinne über das Ohr und die Augen an. Damit ermöglicht die Darstellung im Gegensatz zu anderen, ,zwei Sinnesqualitäten zu einer miteinander verbunden als bewegt zu übermitteln“ (Wyss 2011: 287). Die Kombination verschiedener Sinnesmodalitäten und Zeichen (Ton, Musik, gesprochene Sprache, Bilder) mündet in eine narrative Mehrschichtigkeit. Daraus erklärt sich, dass eine rein 
strukturelle narrative Analyse unzureichend ist, da Synergien aus der Mehrschichtigkeit, wie die Aufwertung und Potenzierung des Erzählten durch Bewegtbilder, außen vor blieben (Wyss 2011: 281). So sind es Farben und Töne, Bilder und Worte, die in ein Verhältnis treten, das auf einen intensiveren Erlebensprozess und eine spezifische Vergegenwärtigung gerichtet ist (Holly 2004: 125). Unter dieser Annahme ging es in der Untersuchung der Gründergeschichte darum, das Zusammenspiel der audio-visuellen Gestaltungsmittel zu untersuchen, um erzählstrategische Vorzüge zu benennen, die diese Realisierung anbietet. Hierfür beschreibe ich markante Merkmale der Aufmachung und greife zur Verdeutlichung einzelne Sequenzen exemplarisch heraus.

Die untersuchte Bewegtbild-Animation macht auf den ersten Blick einen minimalistischen, schlichten Eindruck. Damit ist gemeint, dass die Darstellungskomplexität im Vergleich zu einem Film niedrig wirkt: Die gesamte Farbgebung ist im Rahmen des Corporate Design des Unternehmens gehalten. Der Protagonist trägt einen blauen Anzug und der Hintergrund, vor dem sich die Bilder bewegen, ist einfarbig in cremeweiß. Durch den ,reizarm“ gestalteten Hintergrund stehen allein die animierten Objekte im Vordergrund.

Ähnlich schlicht verhält es sich mit der Bewegungsrichtung der animierten Objekte. Sie ist entweder an der horizontalen oder der vertikalen Bildachse ausgerichtet. So sind die Bewegungen vorhersehbar, erleichtern und lenken die Rezeption und stellen ebenfalls die animierten Objekte in den Mittelpunkt der Aufmerksamkeit:

(Bsp.) Bewegungsrichtung von Objekten in der audiovisuellen Gründererzählung von Siemens

\begin{tabular}{l|l}
\hline $\begin{array}{l}\text { Bsp. horizontale Bewegungsrichtung im } \\
\text { Bewegtbild: } \\
\begin{array}{l}\text { Beginn der Reise nach Russland,00:04 } \\
\text { (Korpusquelle: SIEMENS_564_WS) }\end{array}\end{array}$ & $\begin{array}{l}\text { Bsp. vertikale Bewegungsrichtung im } \\
\text { Bewegtbild: } \\
\text { Der Lift als innovative Leistung des } \\
\text { Gründers, 01:29 } \\
\text { (Korpusquelle: SIEMENS_569_WS) }\end{array}$ \\
\hline$\longrightarrow$
\end{tabular}


Die animierten Elemente selbst zeigen wenig Eigenbewegungen. Personen können Schritt- oder Winkbewegungen ausführen, verfügen jedoch über keine mimischen Regungen oder Sprechbewegungen. Häufig gleiten sie jedoch unbewegt von einem Punkt zum nächsten, erscheinen und verschwinden oder werden hinein- und herausgezoomt. Diese Merkmale verdeutlichen, dass hier kein klassisches Trickfilmformat vorliegt. Die „Verfremdung“ der Bewegung akzentuiert die eingesetzten Bilder.

Die Bewegtbilder stehen in der Gründererzählung häufig symbolisch für das mündlich Wiedergegebene. Dadurch wird die Animation abwechslungsreich und regt zum Schmunzeln an. Hierzu folgendes Beispiel:

(Bsp.) Zusammenspiel von visuellen und sprachlichen Elementen in der Gründererzählung von Siemens

\begin{tabular}{|c|c|c|}
\hline $\begin{array}{l}\mathrm{N} / \text { min. } \\
\text { Merkmale }\end{array}$ & $\begin{array}{l}\text { Standbild, Kapitel 7: 00:08-00:11 } \\
\text { (Korpusquelle: SIEMENS_568_WS) }\end{array}$ & Ton: Sprachtext \\
\hline $\begin{array}{c}10 / 00: 08- \\
00: 10\end{array}$ & I & Im Hintergrund spielt Klaviermusik: \\
\hline $\begin{array}{l}\text { Merkmal: } \\
\text { Die Glüh- } \\
\text { birne fällt } \\
\text { der Person } \\
\text { von oben in } \\
\text { die Hände. }\end{array}$ & & $\begin{array}{l}\text { 02: E: da gibt es EINmal die glÜcklichen } \\
\text { 03: erfinder, } \\
\text { 04: auf eine Nüroßliche idee stoßen, } \\
\text { Während die Glühbirne nach unten fällt, ist ein Pfeifton zu } \\
\text { hören. }\end{array}$ \\
\hline $11 / 00: 11$ & & 05: und damit REICH und berÜhmt werden; \\
\hline
\end{tabular}

Der abgebildete glückliche Erfinder hält in Standbild 10 eine überdimensionale, leuchtende Glühbirne in den Händen. Sie fällt ihm von oben unvermittelt in die Hände. Symbolisiert die Glühbirne die Erfindung, ist es die Fallbewegung von oben, die symbolisch die Mühelosigkeit der Erfindung - den Einfall - visualisiert. Die fallende Birne begleitet ein Pfeifton, der den Eindruck eines schnellen und reibungslosen Vorgangs unterstreicht. Im folgenden Standbild 11 indizieren die zwei Fotoapparate Ruhm und Reichtum, da sie für öffentliches Ansehen und Interesse stehen. Sprache, Bewegtbild und Ton gehen auf den unterschiedlichen Sinnesebenen eine Verbindung ein, die die Rezipient/innen dazu anregt, die mündlichen Inhalte mit der humorvollen Visualisierung anzureichern. 
Als Konsequenz der horizontalen und vertikalen Bewegungsmuster können in der Animation Objekte ineinander übergehen, um daraus etwas Neues und Größeres entstehen zu lassen (Potenzierung durch Visualisierung). Diese Technik findet sich in dem folgenden Beispiel über den Zusammenschluss der bis zu diesem Zeitpunkt international verteilten Siemens-Unternehmen zu einem Gesamtunternehmen (unten von Standbild 16-18). In dem Beispiel verweisen die horizontalen, roten Pfeile auf die Bewegungsrichtung. Sie sind relevant, da so neben den Objekten auch die Personenbewegung im Raum das Zusammenrücken der Familienmitglieder für das Großunternehmen symbolisiert. Die Sequenz schließt zeitlich mit einem Zeitsprung an die vorherige Geschichte an. Die im oberen Teil des 12. Standbildes stehende Jahreszahl, zählt deswegen anfangs mit einem tickenden Geräusch rückwärts. Dabei macht das Ticken erst auf die Rückblende aufmerksam (vgl. Aufmerksamkeitslenkung, Lahn/Meister 2013: 266): 
(Bsp.) Potenzierung durch Visualisierung in der Gründergeschichte von Siemens

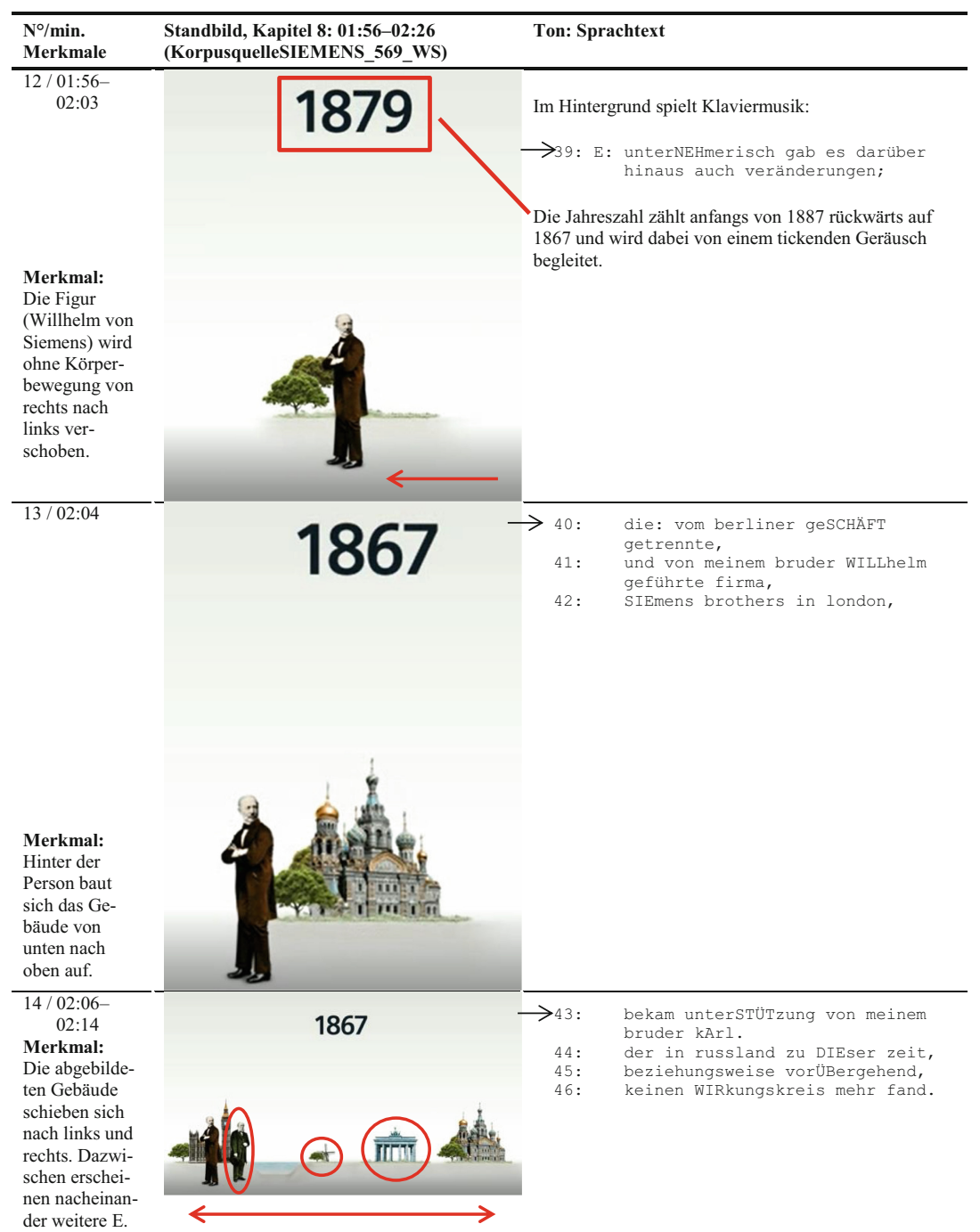




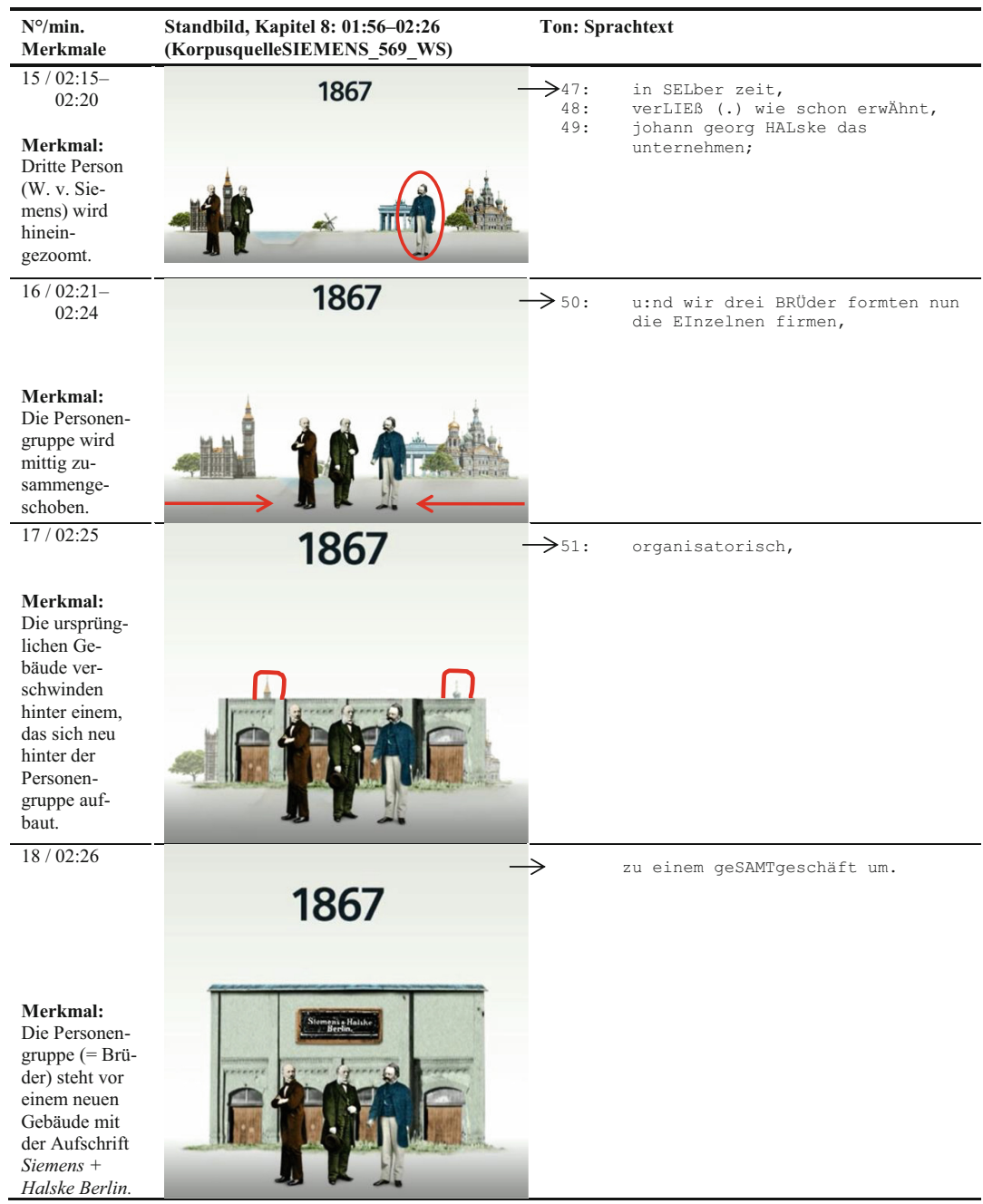

In dem gerade angesprochenen Beispiel werden neben dem Protagonisten Werner von Siemens weitere Personen (Familienangehörige, Geschäftspartner) eingeblendet, zu denen in der Gesamterzählung auch Nebenfiguren (Statisten) zählen. Dadurch dass die menschlichen Figuren sich nicht verändern, keine 
Mimik entfalten, die Gestik und weitgehend die Körpersprache rudimentär sind ${ }^{6}$, stehen die Personen eher im Hintergrund. Es geht vielmehr darum, die erzählten Informationen im Bewegtbild zu verdeutlichen (Illustration) und anzureichern. Weitere Elemente neben Personen sind Gebäude, Gegenstände wie Innovationen (Telegrafenschreiber, Lift u. a.), Landkarten und Dokumente, die Wyss in filmischen Darbietungen „stumme Zeugen“ (ebd. 2011: 296) nennt.

Die bildliche Folge der Szenenwechsel erfolgt synchron zu der mündlichen Erzählung und wechselt entsprechend abrupt von einem Szenario in das andere (jump cuts, Wyss 2011: 283), was den Erzähltext dynamisiert. Lässt man die Animation ohne Ton laufen, erschließen sich die Bewegtbilder nicht, auch wenn die Rezipient/innen bestimmte Elemente aufgrund ihres Weltwissens als zusammengehörig annehmen können ${ }^{7}$. Ändert sich das musikalische Thema, dann kündigt das ein besonderes Ereignis an, z. B. steht der Hochzeitsmarsch für die Heirat. So entsteht eine enge Vernetzung aller aufgewendeten visuellen und auditiven Zeichen, die sich zu einer Gründererzählung verbinden. Durch die „schlichte“ Darstellung trägt das Format der imaginierten Ich-Erzählung des Protagonisten Rechnung, da die Animation den fiktionalen Aspekt widerspiegelt. Das visuelle Stilmittel hierfür ist das ,Spiel mit der Ähnlichkeit und Verfremdung ' von Akteuren. Ähnlichkeit besteht in den Gesichtern der Siemens-Familienmitglieder, während die Verfremdung durch die Animation entsteht. Ein weiterer Effekt der Bewegtbildgestaltung besteht darin, dass die Figur Werner von Siemens immer die gleiche schlichte Kleidung trägt und durch die minimalistische Animation sowohl einen bescheidenen wie auch neutralen Eindruck erweckt. Dieser visuell evozierte Eindruck steht im Kontrast zu dem Erzähltextinhalt, in dem eine Vielzahl von Leistungen zu einem Gründermythos kumulieren.

\subsection{Corporate Blogerzählungen}

Corporate Blogs sind in erster Linie Weblogs - also Webseiten -, die von Unternehmen betrieben werden (vgl. Puschmann 2010: 15). Der Begriff Weblog taucht zum ersten Mal im Jahre 1997 auf. Zu diesem Zeitpunkt benötigt man für das

\footnotetext{
${ }^{6}$ Ebenso verhält es sich mit den Animationen zur Hochzeit, Krankheit oder Familiengründung.

${ }^{7}$ Z. B. wenn in einem Bild eine Person am Schreibtisch sitzt und schreibt und im nächsten Bild ein Dokument zu sehen ist, auf dem,Gesetzt' steht (Korpusquelle: SIEMENS_566_WS). Mit etwas Phantasie kann hier ein Zusammenhang hergestellt werden. Die Information ist allerdings spärlich, da sie nicht viel im Sinne der Gründergeschichte aussagt.
} 
Betreiben von Weblogs in Form von ,per Hand aktualisierten Linklisten“ (Puschmann 2012: 93) noch umfassende technische Kenntnisse über HTML (Hypertext Markup Language), eine Auszeichnungssprache im Internet. Die Bezeichnung ,Weblog ' ist ein Portmanteauwort, das sich aus den Lexemen ,Web' (engl. ,Netz') und ,Log' (von ,Logbuch') zusammensetzt. Häufig findet man in synonymer Verwendung das Kurzwort ,Blog'. Personen, die Weblogs betreiben, bezeichnet man als Blogger/innen, die sich mehr oder weniger an ein bestimmtes Zielpublikum richten. Das dynamische Netzwerk, das sich aus der Gesamtheit der Verlinkungen aller Weblogs bildet, wird Blogosphäre genannt. Als im Jahr 1999 kostengünstige Software mit vereinfachter Handhabung zur Verfügung stand, nahm die Nutzung und somit auch die Verbreitung von Weblogs erheblich zu. Damit einher ging u. a. die Veränderung von link-intensiven zu aufwendig multimodal gestalteten Weblogs (siehe hierzu Klemm 2018: 12). Für Deutschland bescheinigen Studien ab 2004 ein hohes zweistelliges Wachstum hinsichtlich der Nutzung von Weblogs. $\mathrm{Zu}$ Begin nutzten sie vorwiegend Privatpersonen, um ihre persönlichen Eindrücke und Erlebnisse zu schildern (Fischer 2006: 164 ff.; vgl. ebenfalls Klemm 2018: 11 f.; Rinke 2012: 297 f.; Puschmann 2012: 95 ff.; Schlobinski/Sievers 2005: 53 ff.; Prezpiorka 2003: 3 ff.). Eine allgemein gültige Definition für ,Weblog' existiert nicht, doch herrscht Konsens über formale, technisch bedingte Kennzeichen, die Weblogs prägen (vgl. Puschmann 2012: 96). Dabei handelt es sich um Webseiten,

„die regelmäßig aktualisiert werden und die Beiträge in umgekehrter chronologischer Reihenfolge anzeigen, wobei der aktuellste Eintrag jeweils an erster Stelle steht. Oft werden die Inhalte in Textform bereitgestellt, aber auch Bilder, Videos und AudioDateien sind üblich.“ (Fraas/Meier/Pentzold 2012: 25)

Insbesondere in ihren Ursprüngen waren Blogbeiträge mit umfangreichen Linklisten (Blogrolls) angereichert (Klemm 2018: 11), die auf andere Webseiten verweisen. Zusätzlich haben Blogbetreiber/innen die Option, eine Kommentarfunktion einzurichten, über die sich Rezipient/innen zu dem Beitrag äußern können. Das verdeutlicht zugleich, dass die Blogbetreiber/innen eine hohe „Kontrollmöglichkeit“ (Fischer 2006: 179) innehaben, indem sie nicht nur über die Beitragsgestaltung, sondern ebenso über die Kommentarveröffentlichung bestimmen. Sind Blogs thematisch besonders umfangreich, können die Blogbetreibenden die Beiträge zusätzlich in thematische Kategorien unterteilen. Die Kategorien stellen sich den Rezipient/innen als Hyperlinks dar, mit deren Hilfe sie unverzüglich auf das gewünschte Themengebiet navigieren. 
Die wachsende Popularität von Weblogs ist Unternehmen nicht verborgen geblieben, sodass sie Blogs in den letzten Jahren zunehmend mehr als PRInstrument für sich entdeckt haben (Klemm 2018: 12; Rinke 2012: 300; vgl. auch Zerfaß 2005: 5; Efimova 2007). Jochen Mai stellte in seiner Studie (2014) über die DAX-30-Unternehmen fest, dass jedes zweite Unternehmen über einen Corporate Blog verfügt und im Durchschnitt zwei Artikel pro Woche (meist Montag und Mittwoch) bloggt (Mai 2014). Blogs werden allgemein positive Eigenschaften zugeschrieben, die Unternehmen sich zu Nutze machen wollen. Ebert (2015) fasst solche Erwartungen folgendermaßen zusammen: An erster Stelle steht, dass „Pressemitteilungen im Stil des objektiven Nachrichtenjournalismus [...] Gefahr [laufen], als „oberflächlich“, „uniform“ und „distanziert“ gedeutet zu werden“ (ebd. 2015: 501). Beiträge in Corporate Blogs können dagegen von den Anforderungen an Nachrichten abweichen und somit subjektives Erleben in den Mittelpunkt stellen. Aufgrund der Erlebnisperspektive ist es neben ausgebildeten-journalistischen Mitarbeiter/innen ebenfalls semi- oder nicht-professionell ausgebildeten Mitarbeiter/innen (Laienblogger/innen) möglich, Beiträge auf Corporate Blogs zu veröffentlichen. So werde zum einen die journalistische Distanz überwunden und zum anderen der Eindruck von InsiderEinblicken vermittelt, der die Inhalte authentifiziert (vgl. ebenso Zerfaß 2005: 3). Damit streben Corporate Blogbetreiber/innen nicht nur an, ihr thematisches Angebot auszubauen (z. B. Einblicke hinter die Kulissen von Veranstaltungen, zu Arbeitsfeldern oder zur Produktion) und die Aufmerksamkeit der Zielgruppen auf sich zu lenken, sondern ihrem Unternehmen auf diesem Weg ein Gesicht zu geben. Die erforderliche Kernkompetenz von Blogger/innen sieht Klemm (2018: 14) in einem „kontinuierlichen (!) Erzählen“8, dass die Blogger/innen sowohl sprachlich wie auch (audio)visuell leisten.

In den Anfängen beschäftigte sich die Linguistik mit grundsätzlichen Themen der computervermittelten Kommunikation (vgl. Lasch 2015: 306, siehe Abschn. 4.2), beispielsweise mit der Frage, inwiefern das Internet eine neue Sprache bedingt (Androutsopoulos 2007; Marx/Weidacher 2014: 91 ff.; Dürrscheid/ Brommer 2009; weitere Schlobinski/Sievers 2005; Puschmann 2009: 59; 2010). Im Rahmen dieser Forschung wurden sprachliche Phänomene expliziert, die für diese Arbeit gewinnbringend sind, da das Erzählen in den erhobenen Corporate Blogerzählungen überwiegend auf der sprachlichen Vermittlung beruht. Die Ausführungen in diesem Kapitel richten sich daher nicht auf eine weitere Untersuchung dieser Phänomene (wie bspw. in Schlobinski/Sievers 2005: 53 ff.;

\footnotetext{
8 Ähnlich bei Rinke (2012) im Sinne der narrativen Selbstunternehmung in Anlehnung an Bröckling (2007) oder bei Puschmann (2010) im Zusammenhang mit personal blogs.
} 
Puschmann 2010) oder des Dialogs (z. B. bei Klemm 2018), sondern auf spezifische narrative Praktiken, die auf zwei unterschiedliche Autortypen zurückzuführen sind. Ausgehend von den Merkmalsbereichen, die ich für die Untersuchung des narrativen Vermittlungsvorkommens als relevant erachte (Abschn. 5.2.2), fokussiere ich Merkmale der Beschreibungsebene ,narrative Themenentfaltung " in Verbindung mit der Beschreibungsebene ,Text-Realisierung'. Da es zu den Konventionen von Blogbeiträgen gehört, dass sie eine/n namentlich identifizierbare/n Autor/in haben (Puschmann 2012: 102, 2010), ist in diesen Fällen eine eindeutige Zuordnung der Erzählperspektive und darüber der Involviertheit der Erzähler/innen in das Unternehmensgeschehen im Interesse der Unternehmen. Halten sich Unternehmen nicht an diese Konvention, entsteht der Eindruck von Manipulation, wie das bspw. Puschmann (2009) in seinem Artikel Lies at WalMart beschreibt ${ }^{9}$. Hinsichtlich der Beiträge ist ,,[d]er Text an sich das wichtigste variable Element des Blogs“ (Puschmann 2012: 103). Insbesondere die Länge und Komplexität der Beträge variiert in den erhobenen Daten - was ich auf zwei unterschiedliche Autorengruppen zurückführe. Im Allgemeinen handelt es sich bei den archivierten Blogerzählungen - allerdings im Falle von Rewe und Siemens nur bedingt - um ausgebaute Langformen, die einen Umfang von mehreren „Seiten“ aufweisen. Unabhängig vom Umfang der Erzähltexte ist jedoch allen Beiträgen gemeinsam, dass sie den prototypischen narrativen Strukturelementen einer geschlossenen Erzählung entsprechen, wie sie unten abgebildet sind (angelehnt an Brinker et al. 2014: 65 f., in der Abbildung innerhalb der Ellipsen). Hinsichtlich der Strukturelemente können die Erzählungen optional mit weiteren Elementen angereichter werden (graue Kästen) (Abb. 9.5):

\footnotetext{
${ }^{9}$ In dem Artikel beschreibt Puschmann, wie die Einheit von Blogger/in und Autor/in in Beiträgen auf dem Blog Life At Wal-Mart inkonsistent war. Das wurde dem Blog zum Verhängnis, da vermutlich impliziert werden sollte, dass hier Mitarbeiter/innen über das Arbeitsklima und ihren -alltag erzählen. Tatsächlich handelte es sich um Testimonials. Das sollte verschleiert werden und wurde aufgrund der fehlenden Einheit von Autor/in und Blogger/in offenbar. Das ursprünglich als Corporate Blog ausgezeichnete Blog entlarvte sich so als ein ,fake blog (or flog)“ (Puschmann 2009: 81).
} 


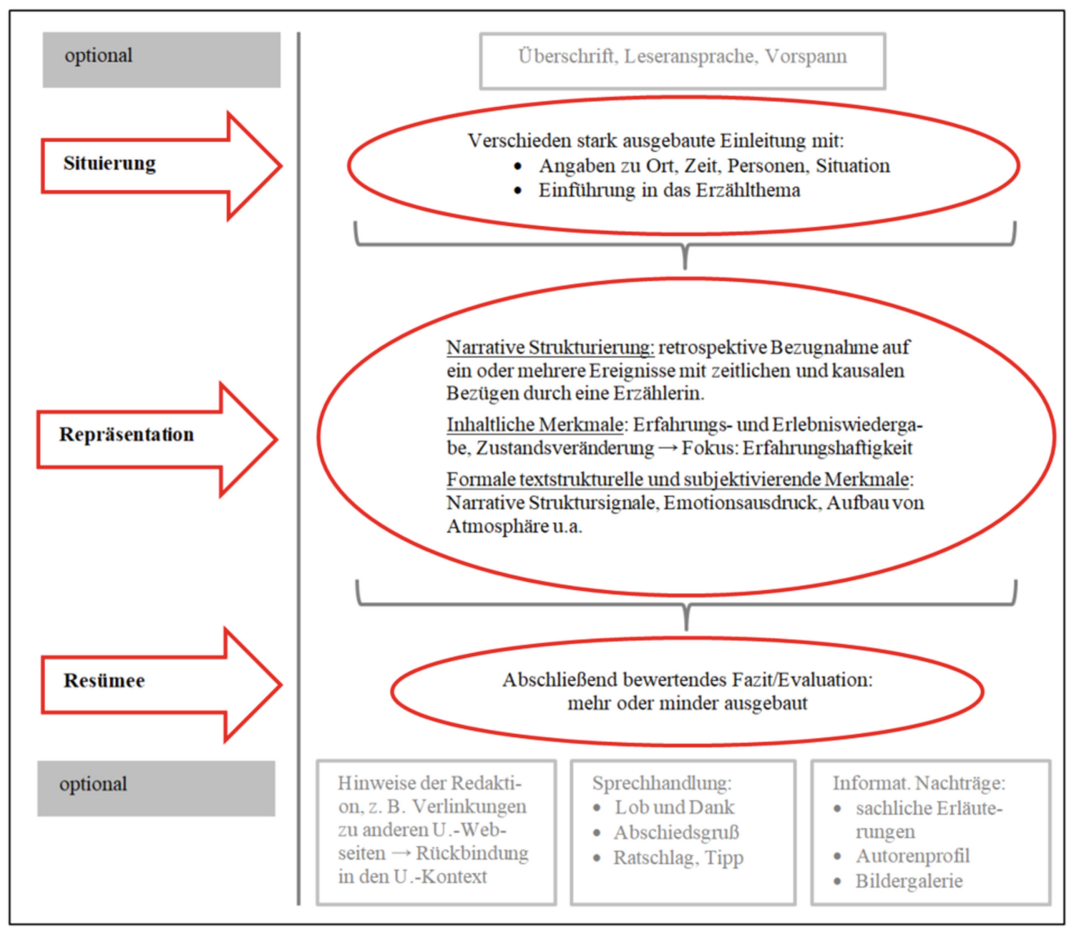

Abb.9.5 Abfolge und Inhalte der narrativen Strukturelemente auf den erhobenen Corporate Blogs

Die Abbildung zeigt, dass die narrative Grundstruktur sich an dem orientiert, was als Weltwissen bei (jungen) Erwachsenen vorausgesetzt werden kann: Einleitung - narrative Ereignisdarstellung - Abschluss. Deutlich wird das an narrativen Gliederungsmerkmalen (Abschn. 5.3.1), die die Erzähltexte strukturieren. Der inhaltliche Fokus liegt auf dem Erleben der Erzähler/innen, das sie durch Emotionen, Gedankeneinblicke und/oder den Aufbau von Atmosphäre vermitteln. Dabei gewichten die Autor/innen - je nach persönlicher Vorstellung einer Erzählung - die in Abschn. 5.4 benannten formalen und textstrukturellen Merkmale unterschiedlich. Bei den optionalen Erweiterungen handelt es sich um Elemente, die je nach Blogbeitrag variabel und in Kombination miteinander auftreten. So findet sich beispielsweise in den Daten häufig eine Kombination von ,Überschrift + Vorspann/Anrede‘. Im Falle der Kombination von ,Überschrift + Vorspann“ 
kann das wie in dem folgenden Beispiel eines Mitarbeiters der Rewe Dortmund aussehen, der Mitglied des Förderkreises Stolz aufRewe ist und einen Beitrag über die Weihnachtsfeier schreibt (Rewe, Blog.Rewe Dortmund, o. J. Korpusquelle: REWE_338_WB):

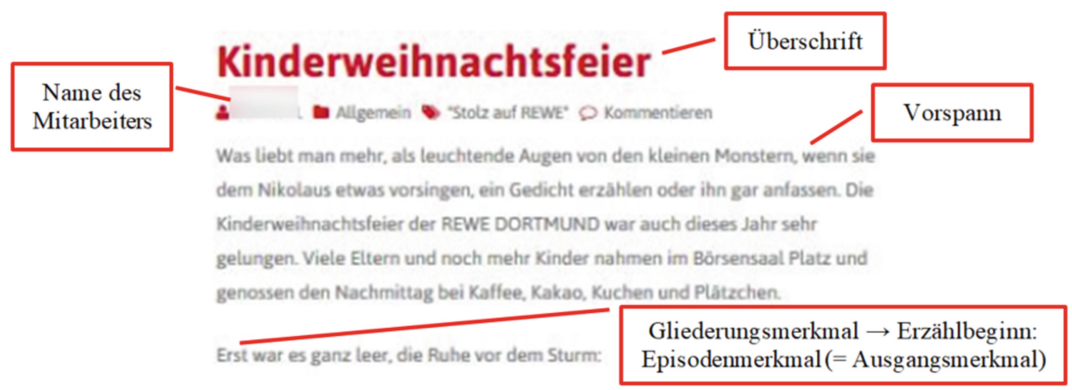

Typografische Markierungen, wie Schriftgröße, -farbe und Fettdruck sowie Absätze als Anfangs- und Endmarkierungen, sind für gewöhnlich abhängig von technischen Voraussetzungen des Corporate Blogs und von Vorgaben des Corporate Design der jeweiligen Unternehmen. Der folgende Auszug eines Eintrags auf dem Traineeblog von Bosch zeigt die Kombination von einer ,Überschrift + Anrede‘. Die Überschrift ist optional. Es existieren uRE, die direkt mit der Ansprache beginnen (Bosch, Traineeblog, 12/2014. Korpusquelle: BOSCH_509_WB):

\section{Einmal "Führen" bitte! Führungsworkshop im JMP}

Hallo!

Was auch immer euch hierhin führt, ich freue mich, dass ihr meinen ersten Blogpost hier im JMP Blog gefunden habt. Ich bin $t=$ und vor einem guten Jahr ins Berufsleben als Teilnehmer des JMPs im Einkauf bei Bosch gestartet. Wie ich zu Bosch gekommen bin und wer ich eigentlich bin lasse ich euch in einem der folgenden Posts wissen.

Jetzt gibt es erst mal einen kurzen Einblick in den Führungsworkshop, ein Seminar das jeder Programmteilnehmer durchläuft und an dem ich letzte Woche teilnehmen konnte.

Erzählbeginn: meta-narrativ

In Bezug auf die narrative Entfaltung und ihre Realisierung ist in dieser Arbeit von Bedeutung, welche narrativen Merkmale mit narrativen Praktiken von Unternehmen in einem Zusammenhang stehen. Diese spezielle Fokussierung nehme ich anhand von Gestaltungsmitteln von zwei unterschiedlichen Autorgruppen auf, die in den Daten für Corporate Blogerzählungen unterschieden werden können. Doch 
zuerst reflektiere ich auf Basis bestehender Forschungsergebnisse über Weblogs und Corporate Blogs grundlegende Merkmale der archivierten Daten, um ihre allgemeinen Charakteristika zu konturieren.

\subsubsection{Charakteristika der Corporate Blogdaten im Spiegel der Forschung}

Untersuchungen über Weblogs sind mittlerweile zahlreich und werden von vielen Fachdisziplinen durchgeführt. Dadurch, dass sich die Forschungsfragen auf sehr unterschiedliche Untersuchungsobjekte und -bereiche richten, sind auch die gefundenen Charakteristika der Weblogs verschieden: „Je nachdem, ob man Blogs als Textsorte, Datenquelle, Instrument des Wissensmanagements, Mittler im öffentlichen Diskurs, Lehr- und Lerninstrument, virtuelle Diskursgemeinschaft oder Ort des persönlichen Ausdrucks betrachtet, erhält man sehr unterschiedliche Charakterisierungen.“ (Puschmann 2012: 99)

Nicht gerade einfacher wird der Überblick dadurch, dass es ,das Blog/den Blog“ im Grunde nicht gibt: „Vielmehr handelt es sich um eine äußerst heterogene Kommunikationsform, die zahlreiche Subkategorien und Textsorten herausgebildet hat" (Klemm 2005: 12). Das schlägt sich in Form breitgefächerter Inhalte und einer beträchtlichen stilistischen Variation auf den einzelnen Blogs nieder (vgl. Puschmann 2012: 100). Daher unterscheidet Puschmann die Orientierung der Forscher/innen nach inhalts- und akteursbezogenen Interessensschwerpunkten (2012: 99). Sprach- und literaturwissenschaftliche Untersuchungen wendeten ihre Aufmerksamkeit vornehmlich auf die generierten Inhalte ${ }^{10}$. Dagegen nähmen bspw. Soziolog/innen, Psycholog/innen oder Kommunikationswissenschaftler/innen eher die Akteure anhand von qualitativen und quantitativen Befragungen in den Blick. Linguistische Analysen befassen sich zum Beispiel mit dem diachronen Wandel des Personal Weblog als internetbasierter Textsorte (Schildhauer 2014), mit Weblogs unter der transmedialen Perspektive (Fraas et al. 2006), Weblogs als Plattform für internetbasierte Wirklichkeitserzählungen von Privatpersonen (Tophinke 2009) und deren Veränderungen im Laufe der Zeit (Tophinke 2017), Weblogs unter einer rhetorischen Perspektive (Klemm 2018) oder unter gattungsbezogenen Fragestellungen (Puschmann 2010, 2012, 2013,

\footnotetext{
${ }^{10}$ Daneben sind insbesondere die Wirtschafts- und Betriebswirtschaftswissenschaften (Zerfaß 2005; Fischer 2006; Kreutzer [2012] (2014)) und die Kommunikationswissenschaften (Przepiorka 2003; Krüger 2015) in dem Themenbereich ,Unternehmen und soziale Medien/Weblog' engagiert.
} 
2009), der Frage nach einer vom Web 2 „geforderten“ dialogspezifischen Umsetzung auf Blogs (Lasch 2015) sowie der narrativen Inszenierung des Selbst in Zeiten der New Economy (Rinke 2012). Bereits diese Aufzählung veranschaulicht, wie breit die Forschungslage zu Weblogs allein im Fachbereich der Linguistik ist. Für die folgende Betrachtung ist eine Differenzierung bezüglich der Frage, ob die Forschungsergebnisse sich nun explizit auf das Erzählen oder Corporate Blogs richten, nur bedingt relevant, da die Ergebnisse Merkmale von bestimmten Weblogs und Blogbeiträgen herausstellen, die kontrastiv oder ergänzend genutzt werden können, um Charakteristika der untersuchten Corporate Blogs und ihrer Beiträge zusammenzufassen. Im Anschluss daran greife ich ausgewählte spezifische Umsetzungsoptionen für die Daten heraus.

Ein Merkmal der erhobenen Corporate Blogdaten, das im Spiegel der Forschungsliteratur deutlich wird, ist die "Geschlossenheit“ der Blogosphäre. So stellen bspw. Fraas et al. (2006) in ihrer Untersuchung zu Pundit Weblogs ${ }^{11}$ fest, dass es gerade die extreme Vernetzung mit anderen Webseiten unterschiedlicher Art ist, die den Reiz und Mehrwert der Blogs ausmacht. In den untersuchten Corporate Blogbeiträgen besteht nur eine bedingte Vernetzung. Ist eine Vernetzung mittels Verlinkungen gegeben, dann handelt es sich um Zusatzinformationen innerhalb der unternehmenseigenen Internetangebote ${ }^{12}$.

Ein zweites Merkmale, das sich in den Daten abzeichnet, ist eine geringe Interaktion als Reaktion auf die Blogbeiträge. Diesbezüglich hält Puschmann (2012: 108) im Ergebnis seiner Arbeiten über Corporate Blogs fest, dass ,auf der Mehrheit der Blogs [die Beiträge] wenig bis gar nicht kommentiert" werden. Auch Mai (2014) beobachtet in seiner Studie über Corporate Blogs der DAX-30Unternehmen, dass ein „Kommentarproblem“ bestehe, da zwei von drei Blogs unkommentiert bleiben, während der Rest im Schnitt zwei Kommentare erhält. Auch Lasch (2015) beobachtet die „Kommentararmut“ in seinem Beitrag zur ,Dialogizität auf Corporate Blogs'. Entgegen dem vornehmlich benannten positiven Effekt von Corporate Blogs, ,mit unterschiedlichen Stakeholder in einen Dialog zu treten“ (Kreutzer 2012: 346, nach Lasch 2015: 307), sieht Lasch in seinen Daten, dass im Grunde kein Dialog zustande kommt, selbst wenn die Blogger/innen sich scheinbar dialoginitiierend äußern. Als Ursache hierfür

\footnotetext{
${ }^{11}$ Blogger/innen von Pundit Weblogs haben einen ,,ausgeprägten Enthüllungs- und Überwachungsanspruch“ (Fraas et al. 2006: 10). Sie ,verstehen sich selbst als Experten für bestimmte Themen oder auch als globale Beobachter und Kritiker von Medien und Politik“ (ebd.).

12 Ähnlich wie das Fraas et al. (2006: 24) im intermedialen Vergleich für Verweise in Printmedien beobachten.
} 
benennt er die instrumentelle Orientierung der externen Unternehmenskommunikation (Stabilisierung eines spezifischen Fremdbildes/Image (Lasch 2015: 300)) und die damit verbundenen zugrunde gelegten monologisch und unidirektional ausgerichteten Kommunikationsmodelle, die die Rezipient/innen zu bloßen Empfänger/innen einer Botschaft machen. Interaktive Aushandlungsprozesse stehen nach Lasch (2015) quer zu der instrumentellen Vorstellung der Unternehmenskommunikation, ein einheitliches Fremdbild zu etablieren, „das möglichst viele Merkmale des inszenierten bzw. ausgehandelten Selbstbildes aufweist" (ebd. 2015: 306). Interaktion geht in diesem Fall mit einem möglichen Kontrollverlust über die Aushandlungen und deren Wirkung auf das Unternehmen einher. Diese Ergebnisse decken sich weitgehend mit Beobachtungen in den erhobenen Daten ${ }^{13}$. Ausschlaggebend dafür sind in erster Linie technische Voraussetzungen. Das bedeutet zum einen, dass nicht immer eine Kommentarfunktion freigeschaltet ist, und zum anderen, nicht alle einen Kommentar abgeben können. Im ersten Fall wird der Corporate Blog lediglich als Informationsinstrument zur Verbreitung einer Botschaft genutzt. Im zweiten Fall besteht die Frage, ob nur Unternehmensmitglieder oder auch externe Personen den Beitrag kommentieren können. Je nach dem kann es sein, dass externe Kommentartoren „Hürden“ nehmen müssen. Das bedeutet, dass die Unternehmen für einen Kommentar entweder eine Registrierung voraussetzen oder die Kommentator/innen sich über ein soziales Netzwerk einloggen müssen. Grundsätzlich ist die Responsivität mittels Kommentaren in den Daten gering und wenig facettenreich: Entweder die Kommentare kommen nur aus der Unternehmensbelegschaft, die den entsprechenden Blogbeitrag lobt ${ }^{14}$, seltener um eigene Erfahrungen ergänzt ${ }^{15}$ oder etwas nachfragt ${ }^{16}$. Manchmal reagiert auch nicht der/die Blogger/in, sondern eine

${ }^{13}$ Ausnahmen finden sich auf dem Corporate Blog von Daimler. Hier kommt es mitunter zu Kommentarerwiderungen zwischen den Mitarbeiter/innen.

${ }^{14}$ Z. B. ,sehr geehrter Herr [...], Hut ab vor diesem gelungen Beitrag [...]“ (Kommentar, Das Blog, 2014-08-01. Korpusquelle: DAIMLER_052_WB).

${ }^{15}$ Z. B.: ,Vielen, vielen Dank für diesen tollen Artikel! Ich war zwar nur 3 Monate in diesem sagenhaft spannenden Land tätig, aber kann die Eindrücke dadurch bereits sehr gut nachvollziehen [...]“ (Kommentar, Das Blog, 2014-03-14. Korpusquelle: DAIMLER_062.1_WB).

${ }^{16}$ Z. B.: „Hallo Frau [...], ich habe ebenfalls Interesse an einem Praktikum in Südafrika und ein paar Fragen an Sie. Können sie mich hierzu kontaktieren?“ Antwort: ,[...] Kontaktieren Sie mich doch einfach unter: [...]“ (Kommentar, Das Blog, 2015-08-26. Korpusquelle: DAIMLER_066_WB). „Hallo. Nachdem ich diesen Blog gelesen habe, bin ich sehr an einem Einstieg bei Ihnen interessiert. Können Sie mir sagen, welche Möglichkeiten Sie für Studenten anbieten?" (Kommentar, Blog Telekom, 2015-08-13. Korpusquelle: DTKOM_828_WB). 
Person aus der Unternehmenskommunikation, die ein Kontakt- oder Informationsangebot macht ${ }^{17}$ (das betrifft insbesondere Das Blog von Daimler). Ist die Kommentarfunktion für unternehmensexterne Personen geöffnet, finden sich in den Kommentaren vereinzelt positive Rückmeldungen von Personen, die an den erzählten Inhalten in irgendeiner Weise teilhatten ${ }^{18}$ (z. B. Teilnehmer/innen von Recruiting Veranstaltungen oder Gewinner/innen von sozial-gesellschaftlichen Unternehmensangeboten). Darüber hinaus nutzen manche Rezipient/innen die Kommentarfunktion, um Informationen zu erfragen, auf die jedoch selten eine Antwort seitens der Blogger/innen folgt ${ }^{19}$. Im Allgemeinen ist zu bedenken, dass Kommentare prinzipiell hinsichtlich ihrer Authentizität und Urheberschaft kritisch behandelt werden sollten. Lasch (2015) unterstreicht diese Einschätzung über Corporate Blogs, nach der es sich um einen seitens der Unternehmen stark regulierten Kommunikationsraum handelt (ebd. 2015: 309).

In diesem Zusammenhang deutet sich ein inhaltlich bedingtes Merkmal an, das die erhobenen Corporate Blogerzählungen auszeichnet. Es wird durch die öffentlich diskursive Orientierung bedingt. Sie hat zur Folge, dass keine Themen ausgehandelt werden, sondern auf bereits etablierte öffentliche Themen reagiert wird, um darüber einen imagefördernden Diskurs für sich zu initiieren. Verständlicher wird das im Kontrast zu den Beobachtungen von Fraas et al. (2006: 9). Fraas et al. halten fest, dass die untersuchten Pundit Weblogs - aber auch Weblogs im Allgemeinen - an öffentliche Diskurse anschließen, indem die Blogger/innen öffentliche Themen aufbereiten und weiter bearbeiten. Hier sehe ich einen deutlichen Unterschied zu den von mir erhobenen Daten. Die untersuchten Blogbeiträge nehmen zwar Themen von öffentlichem Interesse auf (siehe Abschn. 7.1.1.2), doch geht es hierbei nicht darum, an den bestehenden Diskurs anzuschließen. Die externe Unternehmenskommunikation initiiert in den Daten somit nicht selbst eine Diskussion, sondern thematisiert eine Reaktion des

${ }^{17}$ Z. B.: ,[...] wow! Im Sinne Lebenslanges Lernen werde ich mir die Bedingungen des Daimler Academic Programm doch auch mal anschauen ...“ $\rightarrow$ Antwort: ,[...] Wir stehen Ihnen auch gern persönlich für Fragen zur Verfügung [...]“ (Kommentar, Das Blog, 2016-01-07. Korpusquelle: DAIMLER_055_WB).

18 „Super Workshop mit tollen Teilnehmern, toller Orga seitens LYM Team und tollen Bloggern“ (Kommentar, Blog Telekom, 2015-11-12). „Sehr cooler Artikel. Bin Gespannt auf die nächsten Events“ (Kommentar, Blog Telekom, 2014-09-15. Korpusquelle: DTKOM_824_WB).

${ }^{19}$ In den erhobenen Blogbeiträgen von E.ON, REWE (da sich das Großunternehmen Rewe aus einem Verbund von Einzelhandelsunternehmen zusammensetzten, existiert kein übergreifender Blog, sondern die Option hängt von der Eigeninitiative der Einzelunternehmen ab), . Bei Bosch, Siemens, Telefonica Deutschland und EnBW finden sich fast keine Kommentare vorhanden, auch wenn eine Kommentarfunktion angeboten wird. 
Unternehmens auf die öffentliche Diskussion. Auf diesem Weg macht sie die eigene Reaktion (z. B. Hilfsangebote) zum öffentlichen Diskursthema (imagefördernde (S)D). Unternehmen besetzen aktiv ein Thema und kontextualisieren es hinsichtlich imagefördernder Aspekte.

Technischer Fortschritt sowie zunehmend professionelle und kommerzielle Interessen von Weblogbetreiber/innen wirken sich ebenfalls auf die Gestaltung der Weblogs aus. Tophinke (2017: 73) benennt verschiedene Veränderungen im Vergleich zu ihrer Untersuchung von 2009 über Weblogs. Darunter fällt bspw., dass die Blogger/innen normorientierter texten und die Kommentarmöglichkeiten eingeschränkter sind. Dadurch kann sich spontanes konversationelles Erzählen in den Kommentarbereichen nicht mehr fortsetzen, das sprachlich-interaktive Geschehen ist kontrollierter und weniger offen. Hinzu kommt nach Klemm (2018: 12), dass sich das „Medien-Establishment“ Weblogs mehr und mehr aneignet. Dadurch verlieren viele Weblogs ihren ,anfänglichen alternativen Charme“ (ebd.) und entwickeln sich „,vom persönlichen Tagebuch zum journalistischen Onlinemagazin“ oder auch zum ,audiovisuellen/multimodalen Hochglanzmedium" (Klemm 2018: 12 f.). Das ist im Grunde ein Trend, dem die externe Unternehmenskommunikation in den erhobenen Corporate Blogs folgt. Zwar ist der Text in den untersuchten Blogerzählungen nach wie vor dominant, doch sind die Texte mit Fotografien, Slide-Shows und/oder audiovisuellen Bewegtbildbeiträgen (z. B. Videotagebuch) aufgewertet. Die Blogger/innen müssen demnach über differenzierte Kenntnisse zur Bild- und Tonbearbeitung verfügen. Die Kontrolle über die eigene Performanz und die Kommentare führen auch in Corporate Blogs zu einer „Gestaltungsmacht“ (Klemm 2018: 13), die Klemm grundsätzlich durch die zunehmende Professionalisierung von Weblogs beobachtet. Es entsteht ein kontrolliert strategisches Selbstmanagement, wie es sich auch in den untersuchten uRE widerspiegelt.

\subsubsection{Datengrundlage: Mitarbeitererzählungen}

Von den zehn ausgewählten Unternehmen verfügen zum Erhebungszeitpunkt bis auf Volkswagen - alle über Corporate Blogs. Allerdings unterscheiden sie sich nicht nur in ihrer Benennung, sondern auch hinsichtlich ihrer Inhalte und zu Teilen hinsichtlich der Adressat/innen (Kolleg/innen, potenzielle Bewerber/innen, 
interessierte Kund/innen u. a. ${ }^{20}$. Damit geht einher, dass die Erzählanlässe breit gefächert sind. Sie berühren alle vier ermittelten Themenfelder (Abschn. 7.2.1.2) und lassen somit - anders als in diskontinuierlichen Erzählungen oder Kompakterzählungen - keine Spezialisierung auf bestimmte Gebiete erkennen. Ein weiteres Merkmal bezieht sich auf den „Zustand“ und die Wartung der Corporate Blogs. Im zeitlichen Rahmen der Datenerhebung wurden fast alle Blogs, insbesondere bezüglich der Bildschirmansicht oder der inhaltlichen Struktur, überarbeitet. Darüber hinaus wurden bspw. bei E.ON inhaltliche Veränderungen vorgenommen, indem ehemalige Blogartikel auf die U.-Homepage gestellt und die bestehenden Blogs zusammengeführt bzw. umbenannt wurden. Hinzu kommen inhaltliche Neuausrichtungen wie beim Blog von Edeka. Der zum Untersuchungszeitpunkt bestehende Blog stellte Vorgänge ,hinter den Kulissen“ des Unternehmens und im Zusammenhang mit Lebensmitteln in den Vordergrund. Dieser Blog existiert heute nicht mehr. Dafür existiert ein neuer Blog, der ausschließlich auf den Bereich ,Karriere' ausgerichtet ist. Auch die Entwicklung des Daimler Blogs fällt auf: Das Unternehmen schloss den Blog am 01.11.2019 mit einer „Grabrede“ (,Es war schön mit dir! Lange Grabreden mögen die Wenigsten [...]“ (Knaus, Das Blog, 2019-10-31)) und kündigt gleichzeitig das Nachfolgeformat an (,Gespannt auf den Nachfolger? In genau einer Woche, am 7. November wird das Daimler-Magazin an den Start gehen [...]“ (ebd.)). Die folgende Tabelle gibt eine Übersicht (Tab. 9.2):

\footnotetext{
${ }^{20}$ Vorschläge darüber, wie Weblogs typologisiert werden können, sind zahlreich. Puschmann (2010: 94) geht in seiner typologischen Klassifizierung von Unterscheidungskriterien wie Sender- und EmpfängerInstanzen sowie verschiedenen kommunikativen Funktionen/unternehmerischen Zielen aus (z. B. ,image“ oder ,recruitment“). Ebenfalls pragmatisch orientiert sie das Quadrantenmodell von community blogs. In der horizontalen Ebene wird auf der Ebene der Autor/innen zwischen Einzelperson oder kollaborativen Inhalten unterschieden. Auf der vertikalen Ebene bewegen sich die Inhalte zwischen persönlich oder spezialisiert im privaten oder öffentlichen Bereich. (Nünning/Rupp 2012: 29 f.) Daneben existieren Kategorisierungen anderer Fachdisziplinen, die sich ausschließlich an der Funktion der Blogs orientieren, wie zum Beispiel: ,Service-Blogs, Campaigning-, Issues-, Product/Brand, Customer-Relation- bis hin zu Crisis-Blogs“ (Bruhn 2010: 479, nach Klemm 2018: 307). Da das Kriterium für Corporate Blogs in erster Linie die Verwendung durch ein Unternehmen ist und grundsätzlich narrative Praktiken zur (S)D den Untersuchungsgegenstand bilden, ist ein Kategorisierungsversuch verzichtbar. Einziges Ausschlusskriterium ist die explizite Produktwerbung, da es vorrangig um die (S)D hinsichtlich immaterieller Unternehmenswerte geht.
} 
Tab. 9.2 Veränderungen an den untersuchten Corporate Blogs am Ende des Projektes

\begin{tabular}{|c|c|c|}
\hline Unternehmen & Corporate Blog Bezeichnung & Status \\
\hline EnBW & EnBW Kundenblog & $\begin{array}{l}\text { Überarbeitet: neue Ansicht und } \\
\text { Struktur } \\
\text { Blogbenennung unverändert }\end{array}$ \\
\hline E.ON & $\begin{array}{l}\text { Blogverbund aus zwei Blogs: } \\
\text { \#energized und \#onedaywith }\end{array}$ & $\begin{array}{l}\text { Überarbeitet: Artikel wurden zum } \\
\text { Teil auf die U.-Homepage in die } \\
\text { Rubrik ,Nach-haltigkeit‘ gestellt. } \\
\text { Umbenennung: ,Neue Energie } \\
\text { Blog‘ }\end{array}$ \\
\hline Edeka & $\begin{array}{l}\text { Blog: Hinter den Kulissen von } \\
\text { Edeka }\end{array}$ & $\begin{array}{l}\text { Inhaltlich neu ausgerichtet: } \\
\text { Ausbildungsblog Edeka Südwest }\end{array}$ \\
\hline Rewe & Blog. Rewe Dortmund & Blogbenennung unverändert \\
\hline Bosch & Bosch-Trainee-Blog & $\begin{array}{l}\text { Aufgelöst und thematisch in die } \\
\text { U.-Homepage integriert }\end{array}$ \\
\hline Siemens & Blog.Siemens & $\begin{array}{l}\text { Überarbeitet und thematisch in } \\
\text { seperate Blogs unterteilt: Products, } \\
\text { Thought Leadership, Academic, } \\
\text { Corporate }\end{array}$ \\
\hline $\mathbf{V W}$ & - & - \\
\hline Daimler & Das Blog & $\begin{array}{l}\text { Ersetzt durch Magazin für Mobilität } \\
\text { und Gesellschaft }\end{array}$ \\
\hline Dt. Telekom & Blog.Telekom & $\begin{array}{l}\text { Überarbeitet } \\
\text { Blogbenennung unverändert }\end{array}$ \\
\hline Telefonica Dtl. & Blog: Think Big Blog & $\begin{array}{l}\text { Unverändert } \\
\text { Blogbenennung unverändert }\end{array}$ \\
\hline
\end{tabular}

Insgesamt sind ca. 71 Prozent $^{21}$ der erhobenen Corporate Blogdaten uRE in der ersten Person (= homodiegetische Erzählungen), die - insofern sie keinen Gastbeitrag darstellen - von Mitarbeiter/innen verfasst worden. Das verwundert nicht, da die Perspektive einer/s Ich-Erzähler/in, die aus der Übereinstimmung von Autor/in und Blogger/in (= Erzähler/in) resultiert, zu den konventionalisierten Eigenschaften von Weblogs gehört (vgl. Puschmann 2012: 103). Das folgende Diagramm veranschaulicht die Verteilung der Daten im Primärkorpus, und welchen Umfang Mitarbeitererzählungen in der jeweils unternehmensspezifischen Aufstellung einnehmen (Abb. 9.6):

${ }^{21}$ Absolute Zahl der Daten im Primärkorpus 279, davon sind 198 Mitarbeitererzählungen. 


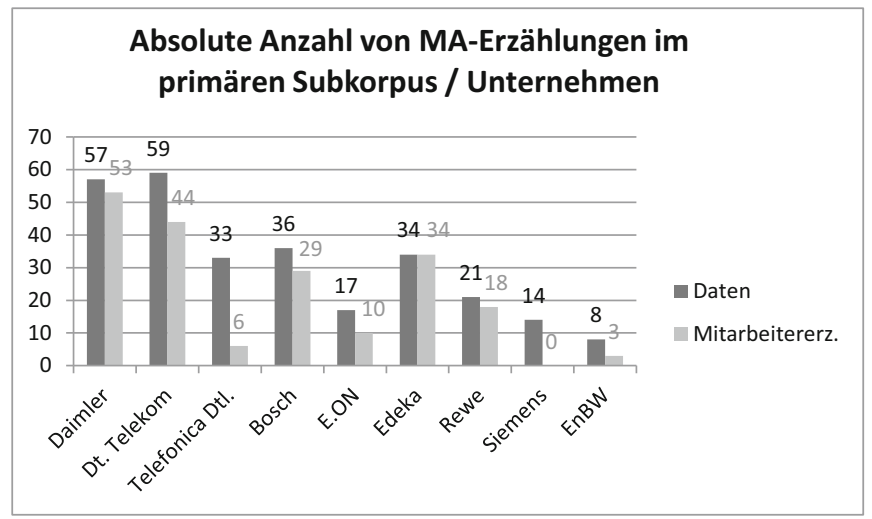

Abb.9.6 Absolute Zahl der Daten im primären Subkorpus mit Anzahl der Mitarbeitererzählungen

Nach der Grobanalyse verblieb ein primäres Subkorpus mit 169 Daten (ca. 60 Prozent des Primärkorpus), das den Datenpool für die sequenzielle Feinanalyse beinhaltet (siehe Abschn. 6.1). Ausgehend von der Relevanz, die Mitarbeitererzählungen in dem Korpus rein quantitativ besitzen, wurde ein sekundäres Subkorpus erstellt. Es beinhaltet zum einen uRE von professionell-journalistisch geschulten Erzähler/innen und zum anderen von Mitarbeiter/innen, die sich nicht als Teil der Unternehmenskommunikation ,ausweisen“. Die leitende Frage ist, welche charakteristisch narrative Praktiken Mitarbeitererzählungen ausmachen. Dabei muss berücksichtig werden, dass es sich um Erzähler/innen mit unterschiedlichem Hintergrundwissen handelt. Im Sinne der qualitativen Forschung habe ich aus dem primären Subkorpus 46 Beispiele ausgewählt, die die entsprechenden Merkmale prägnant veranschaulichen. Die Verteilung über die Unternehmen gestaltete sich wie folgt (= sekundäres Subkorpus zur sequenziellen Feinanalyse) (Abb. 9.7): 


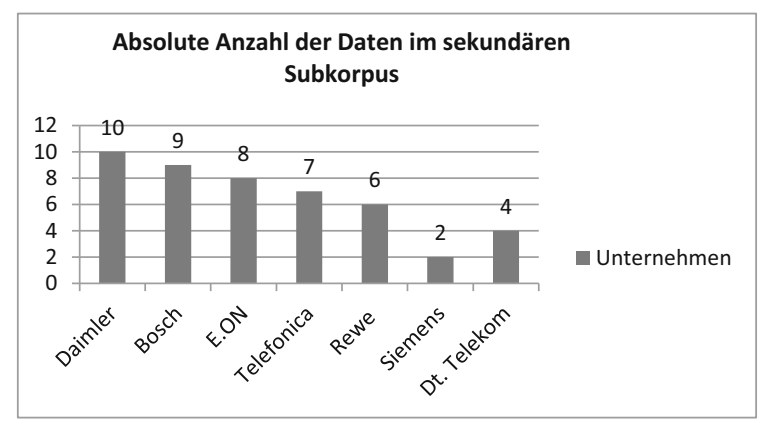

Abb.9.7 Absolute Anzahl der Beispiele im sekundären Subkorpus zur sequenziellen Feinanalyse von Mitarbeitererzählungen auf Corporate Blogs

Das Korpus unterliegt aufgrund der qualitativen Erhebung Selektionsprozessen, die durch die persönliche Wahrnehmung und das entwickelte Verortungsschema beeinflusst werden. Das ist sicher ein Grund für das vermehrte Aufkommen von Mitarbeitererzählungen in dem Korpus. Doch unabhängig von den vorliegenden Daten spielen Mitarbeiter/innen für das bespielen von Corporate Blogs eine bedeutende Rolle (vgl. Abschn. 7.2.2.1). Insbesondere weil sie sich durch die persönliche Erzählperspektive auszeichnen und den Rezipient/innenen dadurch Authentizität suggerieren ${ }^{22}$.

\subsubsection{Zwei Autorengruppen: das Dilemma erzählender Mitarbeiter/innen}

Soll ein Corporate Blog sich von anderen Veröffentlichungen eines Unternehmens unterscheiden, indem sich das Unternehmen auf dem Blog von seiner ,menschlichen“ Seite präsentiert, rücken Mitarbeiter/innen in den Mittelpunkt der Aufgabenerfüllung. Das betrifft zum einen professionell-journalistische Autor/innen,

\footnotetext{
${ }^{22}$ In den Literaturwissenschaften fassen Klein/Martinez (2009) solche Erzählungen als Wirklichkeitserzählungen: „Anders als in den erfundenen Geschichten der Literatur bezieht man sich in diesen Erzählungen direkt auf unsere konkrete Wirklichkeit und trifft Aussagen mit einem spezifischen Geltungsanspruch: > So ist es (gewesen)<. Solche Erzählungen mit unmittelbarem Bezug auf die konkrete außersprachliche Realität nennen wir Wirklichkeitserzählungen“ (ebd. 2009: 1).
} 
die entweder als Teil der externen Unternehmenskommunikation oder als Freiberufler/innen im Auftrag des Unternehmens tätig werden (projektspezifisch angeworbene Blogger wie bspw. Leave-Your-Mark Blogger/innen oder Netztourblogger/innen von der Dt. Telekom). Zum anderen betrifft das nicht-professionelle Autor/innen, nämlich Mitarbeiter/innen aus anderen Abteilungen der Unternehmen, die sich bereiterklären einen Beitrag über ein bestimmtes Ereignis, Erlebnis oder eine Erfahrung zu verfassen (Laienblogger). Mai (2014) empfiehlt Vielfalt für Corporate Blogs. Das bezieht er nicht nur auf die Themen, sondern auch auf die Autor/innen, die sich im Idealfall mit Klarnamen und Autorprofilen ausweisen. Finden sich situativ keine nicht-professionell geschulten Mitarbeiter/innen für einen Beitrag, springen Autor/innen der Unternehmenskommunikation mit Beiträgen ein. Beide Autorengruppen stehen jedoch vor einem spezifischen Dilemma: Während Mitarbeiter/innen mit einem professionell-journalistischen Hintergrund die kommunizierten Inhalte in ihren Erzähltexten zu legitimieren versuchen, geht es bei Autor/innen ohne professionell-journalistischen Hintergrund darum, ihren persönlichen Authentizitätsanspruch ${ }^{23} \mathrm{zu}$ bewahren. Der Anspruch auf Echtheit/Wahrhaftigkeit steht aufgrund unternehmensspezifischer Zielsetzungen, bspw. eine einheitliche Unternehmensidentität bzw. eine positive (S)D zu propagieren, auf dem Prüfstand: Aufgrund der Entstehungsgeschichte von Weblogs als persönliche Tagebücher ist die persönliche und subjektive Perspektive maßgebend (Bedeutung des Individuums und seiner Sicht auf die Sachverhalte).

Schreibt ein/e Mitarbeiter/in, die in einem Unternehmensbereich außerhalb der Unternehmenskommunikation arbeitet, steht und fällt ihre Authentizität/Glaubwürdigkeit damit, inwiefern es ihr gelingt, ihre Individual-Identität innerhalb der Vorgaben der konstruierten Corporate Identity (siehe Abschn. 1.1) einzubringen. Auf der einen Seite zeigt sich der/die Mitarbeiter/in in ihrer Rolle als Unternehmensmitglied und unterliegt in dieser gesetzten Vorgaben und Ansprüchen. Auf der anderen Seite soll sie auf dem Corporate Blog ihre IchIdentität einbringen, um ihre Erzählung zu legitimieren. Das Dilemma besteht darin, dass diese Mitarbeiter/innen den Unternehmen ihre Stimme leihen (vgl. Puschmann 2009: 56 f., 2010: 105). Sie sollen dabei jedoch keinesfalls instrumentalisiert wie eine Marionette oder eine Werbefigur wirken. Das stünde dem Prinzip von Weblogs entgegen, „dass wahre Meinungen und echte, subjektive

${ }^{23}$ Die Verwendung des Terminus „Authentizität“ steht häufig im Zusammenhang mit Wahrhaftigkeit und Echtheit. In diesem Sinne verwende ich ihn hier. Grundsätzlich ist der Begriff aufgrund seiner disziplinübergreifenden Verwendung unscharf. 
Sichtweisen und Erfahrungen erzählt werden“ (Rinke 2012: 302). Zwar mag es sein,

„dass sich Blogger mit dem Unternehmen identifizieren und sich ihre Themen relativ frei wählen können, aber sie müssen stets im Interesse des Unternehmens agieren und dementsprechend loyal sein.“ (Rinke 2012: 301)

Daraus folgt für diese Arbeit, dass sich in uRE dieser Mitarbeiter/innen Merkmale abbilden, die diese Kluft zu schließen versuchen. In diesem Zusammenhang gehe ich in Abschn 9.2.4 darauf ein, wie Authentizität durch den Eindruck von „Unzensiertheit“ belegt werden soll. Das Merkmal ist charakteristisch für Corporate Blogerzählungen, die von nicht-professionell geschulten Autor/innen stammen. Dementsprechend handelt es sich hierbei um eine narrative Praktik von Unternehmensmitgliedern, die dem Authentizitäts-Dilemma der nicht-geschulten Autor/innen entgegenwirken soll.

Das Dilemma mit dem professionell-journalistisch geschulte Autor/innen konfrontiert sind, resultiert ebenfalls daraus, dass sie als Sprachrohr des Unternehmens agieren. In ihrem Fall akzeptieren das die Rezipient/innen, in einem bestimmten Rahmen (insofern nicht darüber hinweggetäuscht wird), da das Aufgabenfeld der Autor/innen aufgrund ihre Rolle bekannt und vorbestimmt ist. Diese Autorengruppe muss jedoch die Aufgabe bewältigen, dass sie sich weniger in Bezug auf ihre persönliche Authentizität als vielmehr in Bezug auf ihre erzählten Inhalte als authentisch auszeichnen. Da die erhobenen Daten in erster Linie keine „harten“ Fakten, sondern vorrangig Immaterielles wie die Haltung eines Unternehmens zu einem Bereich thematisieren, geht es für diese Mitarbeitergruppe darum, die erwartete Echtheit mithilfe von Zitaten zu unterstreichen. Bezogen auf die narrative Realisierung zeigt sich das verstärkt an narrativen Realisierungen, die sich einer Interviewstory (Schröder 2001) über Mitarbeiter/innen / Kolleg/innen annähern. Das narrative Gestaltungsmittel ist hierbei die Redewiedergabe. Diese verlangt den Autor/innen profundere Kenntnisse über das Schreiben ab, die nicht-journalistisch ausgebildete Blogger/innen ad hoc schwerlich umsetzen können. Daher greife ich die Redewiedergabe als weiteres Merkmal für Corporate Blogerzählungen von Mitarbeiter/innen in Abschnitt 9.2.5 auf.

Bei beiden Autorengruppen handelt es sich folglich um Mitarbeiter/innen (neben Gastautor/innen), die offiziell im Auftrag eines Unternehmens erzählen, um es nach außen in einem positiven Licht darzustellen (Zweckorientierung). Ausgehend von dem Dilemma der jeweiligen Autorengruppe gilt es, dass sie ihre URE anhand bestimmter narrativer Praktiken authentifizieren. Um das charakteristische Kriterium der Authentizität von Weblogveröffentlichungen nicht zu 
diskreditieren, kommt es aufgrund der verschiedenen Rollen zu unterschiedlichen narrativen Gestaltungsmerkmalen, auch wenn beide funktional der (S)D zur Förderung des Unternehmensimages unterliegen. In den folgenden Kapiteln differenziere ich daher die Beiträge nach den benannten Autorengruppen, die formal in den Daten anhand von Zusatzinformationen über die Verfasser/innen unterschieden werden.

\subsubsection{Spezifika von Erzählungen nicht journalistisch ausgebildeter Mitarbeiter/innen}

Die Aussage, dass die wichtigste Voraussetzung für authentisches Bloggen damit in Verbindung steht, dass der/die ausgewiesene Autor/in eines Blogeintrags auch der/die tatsächliche Autor/in ist, bezieht Puschmann (2010: 107) in erster Linie darauf, dass überprüfbare ist, dass es sich um eine real existierende Person handel ${ }^{24}$. Doch auch innerhalb des Erzähltextes existieren Indizien, die die Rezipient/innen annehmen lassen, dass Autor/in und Text ,zusammenpassen“. Einer Untersuchung von Efimova/Grudin (2007) zufolge geben Mitarbeiter/innen eines Unternehmens zwei grundlegende Motivationsfaktoren an, warum sie für ihr Unternehmen bloggen: Zum einen liegt ihnen daran die menschliche Seite ihres Unternehmens hervorzuheben. Zum anderen wollen sie zeigen, dass ihre Arbeit von Bedeutung ist und sie stolz auf ihre Tätigkeit sowie ihre Unternehmenszugehörigkeit sind. Ein Corporate Blog macht sie sozusagen ,sichtbar“: „A weblog also gives its authors visibility“ (2007: o. S.). Trotz dieser hehren Ambitionen muss der/die bloggende Mitarbeiter/in für die Rezipient/innen in den Erzählungen sichtbar sein. Eine hohe Motivation allein ist unzureichend. Insbesondere, wenn man davon ausgeht, dass ein gewisses Misstrauen seitens der Leserschaft besteht. In den Daten existieren zwei augenfällige Merkmale, die ich unter diesen Umständen als narrative Praxis im Unternehmenskontext auf der Textebene verstehe. Dazu zählen

a) das Existieren und die Akzeptanz unbeabsichtigter Normabweichungen und

b) der Rückgriff auf narrative Gestaltungsmittel, um die eigene Erzählung zu Individualisieren.

${ }^{24}$ Den diesbezüglich theoretischen Hintergrund für diese Arbeit behandle ich in Abschn. 7.1.2.1 unter dem situativen Kontext. 
Diese zwei Aspekte zielen darauf, erzählende Mitarbeiter/innen ohne journalistische Ausbildung in den uRE als solche erkennbar zu machen. Die damit verbundenen Merkmale sind stark von nähesprachlichen Indikatoren gekennzeichnet, wie sie in Untersuchungen der computervermittelten Kommunikation (CMC) bereits erhoben wurden. Kritisch zu der Anwendung des sprachlichen Nähe- und Distanzmodells von Koch/Oesterreicher (1985) innerhalb CMC äußert sich Androutsopoulos (2007). Unter anderem verweist er auf die „Medienvergessenheit des Ansatzes“ (ebd. 2007: 80), der sich ausschließlich auf „die grafische Realisierungsform des Zeichensystems Sprache bezieht" (ebd.), ohne die Rolle technischer Medien einzubeziehen. Diesbezüglich sieht Androutsopoulos im Internet einen sich für informelle Netzkommunikation charakteristischen Schreibstil entwickeln, den er anhand von vier Kategorien - Versprechsprachlichung, mimisch-kinesische Kompensation, Ökonomisierung, Graphostilistik - als „neue Schriftlichkeit“ (Androutsopoulos 2007: 81) auffasst ${ }^{25}$. Der Autor bezieht sich auf Schmitz (2006: 192), der das Resultat der Entwicklung „,sekundäre Schriftlichkeit" nennt. Diese beruht auf einem spezifischen Verfahren innerhalb der digitalen Medien, das

„gewollt Spontaneität auf der Grundlage von schriftgewohnter Reflektiertheit zulässt oder gar kultiviert. Schreiben nimmt hier einige Elemente auf, die man von mündlicher Kommunikation gewohnt ist, oder ahmt sie mit medienmöglichen Mitteln nach.“ (Schmitz 2006: 192, nach Androutsopoulos 2007: 81)

Sprachliche Merkmale, die in diesem Rahmen erforscht wurden, sind die Grundlage für die Beschreibung der zwei oben benannten Aspekte, deren Funktion darin besteht, Kongruenz zwischen dem/der Autor/in und dem Erzähltext zu implizieren. Im Folgenden zeige ich zuerst charakteristische Abweichungen von Normvorgaben auf und beschreibe im Anschluss daran typische Gestaltungsmittel, die einen ,persönlichen“, kreativ-narrativen Ausdruck unterstützen.

\footnotetext{
${ }^{25}$ Zusätzlich bezieht der Autor neuere Erkenntnisse aus der interaktionalen Stilistik und Soziolinguistik ein, nach denen sich der Text und der Kontext wechselseitig bedingen, was Koch/Oesterreicher in ihrem Ansatz noch nicht mitgedacht haben. Ausgehend von diesen Erkenntnissen formt die Sprache ebenso den Kontext. Androutsopoulos (2007) zieht daraus den Schluss, dass die Sprache der Nähe im Internet ,also nur verstanden werden [kann] als Sprachgebrauch, der nicht nur eine bereits vor der aktuellen Sprechsituation gegebenen, vorausgesetzten Nähe reflektiert, sondern diese Nähe eben aktiv herstellt bzw. erzielt“ (ebd. 2007: 80).
} 


\subsubsection{Abweichungen von textsprachlichen Normvorgaben}

Die textsprachlichen Abweichungen, auf die ich mich beziehe, wären in einem Text, den eine professionell-journalistisch geschulte Person publiziert, nicht und schon gar nicht in diesem Ausmaß akzeptabel. Aus der Forschung der computerbasierten Kommunikation ist vor allem mit Blick auf die quasisynchrone Kommunikation (Chat, SMS u. a.) bekannt, „dass Spontanschreiben und $>$ virtuelle $<$ Kommunikation direkten Einfluss auf die Verwendung des orthographischen Systems haben“" (Schlobinski/Siever 2005: 72; vgl. auch Storrer 2001; Dürscheid/Brommer 2009). Bemerkbar macht sich das an Tipp- und Flüchtigkeitsfehlern, die eine beschleunigte Textproduktion begünstigt (Storrer 2001). Doch da das Bloggen zeitlich asynchron mit einer unidirektionalen Ausrichtung verläuft, ist es Blogger/innen durchaus möglich, ihre Texte zu planen und zu korrigieren. Dennoch stellen Schlobinski/Sievers (2005) fest, dass in Weblogs zahlreiche Flüchtigkeitsfehler auftreten. Texte auf professionellen Blogs weisen zwar ebenfalls Flüchtigkeitsfehler auf, allerdings nicht in dem gleichen Ausmaß: „In den privaten Blogs nimmt die Fehlerquote deutlich zu“ (ebd. 2005: 74). Die Autoren schließen für ihre Daten daraus, dass sowohl die Planungs- als auch die Korrekturphase eingeschränkt ist. In Bezug auf die vorliegenden Daten unterstelle ich, dass die Korrekturen nicht erfolgen, um die Authentizität des/der nicht-journalistisch geschulten Autor/in in ihrem Erzähltext abzubilden. Aufgefunden wurden in den Daten folgende Fehlerquellen, die für eine geschulte Person ungewöhnlich sind: Die Fehlerquellen beziehen sich a) auf die Zeichensetzung (Kommasetzung), b) die Orthografie (Getrennt- und Zusammenschreibung, Großund Kleinschreibung) sowie c) diverse Flüchtigkeitsfehler (Tippfehler, weitere grammatische Fehler). Im Folgenden illustrieren Beispiele diese Fehlerquellen. $\mathrm{Da}$ in einem Satz auch mehrere Fehler auftreten können, markiere ich zu Gunsten der bessere Lesbarkeit ausschließlich die angekündigten:

a) Zeichensetzung: fehlendes Satzzeichen (Komma) zwischen Teil- und Gliedsätzen

(Bsp. 1) „Wie ich zu Bosch gekommen bin und wer ich eigentlich bin (sic) lasse ich euch in einem der folgenden Posts wissen." (Bosch, Traineeblog, 12/2014. Korpusquelle: BOSCH_509_WB)

(Bsp. 2) ,Vor allem als es in die Abteilung Charter Way für mich ging (sic) kam die Sache mit dem Respekt mal so richtige zum tragen!" (Daimler, Das Blog, 2014-08-01. Korpusquelle: DAIMLER_052_WB)

(Bsp. 3) „So sind sie auch weniger gestresst (sic) wenn der starke Monsun den Feierabend hinauszögert, [...].“ (Bosch, Traineeblog, 12/2015. Korpusquelle: BOSCH_491_WB) 
(Bsp. 4) ,Diesen Auslandsaufenthalt zu machen war wahrscheinlich die beste Entscheidung (sic) die ich bisher in meiner beruflichen Laufbahn getroffen habe [...].“ ( Dt. Telekom, Telekom.Blog, 2016-06-12. Korpusquelle: DTKOM_836_WB)

b) Orthografie

Getrennt und Zusammenschreibung

(Bsp. 1) „Den Ehrgeiz Dinge zu verbessern konnte ich bei Philipp sowie bei mir fest stellen (sic), aber [...]“" (Daimler, Das Blog, 2014-06-27. Korpusquelle: DAIMLER_065_WB)

(Bsp. 2), ,[...] viele dieser Unternehmen benötigen eine globalere Aufstellung und interkulturell feingeschliffenere Art, um im Wettbewerb stand zuhalten (sic) und zu wachsen." (Daimler, Das Blog, 2014-03-14. Korpusquelle: DAIMLER_062.1_WB)

(Bsp. 3) ,[...], mich auf diesem Gebiet entsprechend weiter zu bilden (sic), [...].“ (Daimler, Das Blog, 2016-01-07. Korpusquelle: DAIMLER_055_WB)

Groß- und Kleinschreibung

(Bsp. 1) „Nach etwas mehr als 44 Berufsjahren unter Anderem (sic) im Vertrieb, [...].“ (Daimler, Das Blog, 2014-03-09. Korpusquelle: DAIMLER_063_WB)

(Bsp. 2) ,[...], dass wir nicht nur vieles wissenswertes (sic) erfahren, sondern [...].“ (Rewe, Blog. Rewe Dortmund, ohne Datum. Korpusquelle: REWE_337_WB)

(Bsp. 3) „Ende August haben wir angefangen, Daten zu Sammeln (sic), mit den Werken Durchsprache zu halten." (Bosch, Traineeblog, 12/2014. Korpusquelle: BOSCH_493_WB)

c) Flüchtigkeitsfehler

Darunter fallen Tippfehler in Form von Buchstabenauslassungen (Herausfordrung, zu einer spannende Tour, Vorgesetze, konn e) oder Wortdoppelungen (die nette Betreuerin von von der Fundeciòn / Diese Kindertagesstätte wird für die kommenden Wochen unser Arbeitsplatz und sein und wir lernen $[\ldots])$.

Des Weiteren kommen grammatische Fehler wie fehlerhaft verwendete Präpositionen vor (Hygiene-Standards bei den vorhandenen Einrichtungen wurden erhöht / Der Ärzte-, Pflegepersonalaustausch zwischen China und dem Westen helfen in der Verbesserung der vorhandenen Gesundheits- und Pflegeinfrastruktur / Eine weitere Herausfordrung war die Organisation von [...], ein komplexer Prozess, bei dem ich [...] gut unterstützen konnte.). 
Hinzu kommen Fehler, die aus der Satzumstellung im bereits geschriebenen Syntagma resultieren können, wie zum Beispiel Inkongruenzen hinsichtlich des Numerus (Bei dem Betrieb dessen Produkte mich so begeistern und das mich am meisten überzeugt hatte), vertauschte Wörter (Aber lernt das jeweilige Unternehmen draus und nutzt diese Erkenntnis für ein Umdenken? Packt sie (sic; bezieht sich auf die Erkenntnis) es (sic; bezieht sich auf das Unternehmen) als Herausforderung an?) und ,Wort-Überbleibsel“ (Als, ,Leiter" und konnten wir das Projekt nach unseren Wünschen [...] gestalten).

Diese unabsichtlichen orthografischen und grammatischen Abweichungen von textsprachlichen Normvorgaben implizieren, dass die Erzähltexte kein Lektorat der Unternehmenskommunikation durchlaufen haben. Sie sollen dadurch als ,ungeprüft" und frei von einer unternehmensseitigen Einflussnahme erscheinen, da die Fehler durchaus von den Verantwortlichen getilgt werden könnten. Daher können sie als eine Inszenierung des Authentischen gewertet werden. Klemm (2015) stellt in Bezug auf die mündliche Rhetorik auf Weblogs fest, dass ,,[d]er Charme des >Nicht-Ganz-Perfekten < sozialer Medien [...] in der Regel bewahrt [wird]“ (Klemm 2018: 15). Diese Feststellung lässt sich m. E. ebenfalls auf die sprachliche „Unvollkommenheit“ der Erzähltexte von Mitarbeiter/innen in der Rolle als Laienblogger/innen übertragen.

\subsubsection{Gestaltungsmittel eines „persönlich-narrativen“ Ausdrucks}

Prinzipiell ist jede Corporate Blogerzählung an das Corporate Design wie auch an Nutzungshinweise, Grundsätze oder Social-Media-Guidelines des jeweiligen Unternehmens gebunden (siehe Abschn. 7.1.2.1), deren Einhaltung im Regelfall nach Puschmann (2010: 106) von der externen Unternehmenskommunikation überprüft wird. Durch diese Vereinheitlichungen entsteht eine Gleichförmigkeit, die es aufzubrechen gilt, um die individuelle Atmosphäre jeder Erzählung aufrechtzuerhalten. Wenn bereits die typografische Gestaltung der Mitarbeitererzählung weitgehend vorgegeben ist, sind es die sprachlichen Gestaltungsmittel, durch die Erzähler/innen ihren persönlichen Stil hervorheben können. Hierbei können a) sprachliche Gestaltungsmittel des persönlichen Ausdrucks und b) narrationsspezifische Gestaltungsmittel unterschieden werden.

Sprachliche Gestaltungsmittel des persönlichen Ausdrucks, die in den Mitarbeitererzählungen auftreten, schließen insbesondere folgende Phänomene aus der Forschung über computerbasierte Kommunikation ein (vgl. Storrer 2001: o. S.; Schlobinski/Siever 2005: 72; Androutopoulos 2007: 87): 
- umgangssprachliche (lauschig, Bleifuß, gucken, jobben, Blaumann, cool, super $^{26}$ ) und dialektale Ausdrücke (muckelig, schnucken),

- umgangssprachliche Phraseme (an den Start bringen, etwas passt, auf ihre Boliden pinseln, eine Mütze Schlaf),

- emulierte Prosodie (Hmmm ... riecht das lecker hier! I - hmmm... davon liest, sieht und hört man tatsächlich relativ wenig in den Medien.) und Interjektion (wow, hätte ich über diese Dinge [...] nachgedacht [...]),

- mimisch-kinesische Kompensierungsverfahren durch satzfinale Emoticons in der Standardvariante (schriftzeichenbasiert) in expressiv evaluierender Funktion ([...] an denen wir auch Testkochsessions für das Buffet ausübten :-) / Die EM hier zu gucken [...] , naja zumindest bis Kroatien unglücklich gegen Portugal rausgeflogen ist:( / [...] neben der Entscheidung zur Telekom zu gehen natürlich:D / Aber sind wir mal ehrlich, einen Auslandsaufenthalt zu machen hat auch fernab der Arbeit Vorteile! ;-))

Diese Merkmale untersucht die Forschung über Weblogs insbesondere hinsichtlich des audience design, also dem Eingehen der Blogger/ innen auf eine vorausgehende Konzeptualisierung ihrer Leserschaft. Geht man davon aus, dass die Blogger/innen ein breites Publikum erreichen wollen, ist das selbsterklärend ${ }^{27}$. In Anbetracht zielorientierter Blogs - wie dem Corporate Blog - kann das audience design Teil einer ausgefeilten rhetorischen Strategie sein (Puschmann 2013: 94). Eine strategische Ausrichtung berücksichtigt m. E. neben dem audience desgn die Tatsache, dass nicht-journalistische geschulte Mitarbeiter/innen die Erzählungen verfassen. Das strategische Kalkül bedeutet, einen sprachlichen Ausdruck zuzulassen, der authentisch für die Autor/innen erscheint, im Sinne einer locker, natürlich wirkenden uRE (vgl. Puschmann 2012: 103). Die Sprache wird sozusagen zum Indikator für eine/n individuelle/n Autor/in, indem sie eine Art persönlichen Stil impliziert - wenn man wie Eroms (2014) einen persönlichen Stil ,als adäquate, nicht aufgesetzte, individuell geprägte Äußerungsform“ (Eroms 2014: 35) betrachtet. Insbesondere der Aspekt, dass die sprachliche Gestaltung nicht ,aufgesetzt“ erscheint, soll den persönlichen Stil verstärken. Die Tatsache, dass die sprachlichen Mittel in jeglichen uRE auftreten, sehe ich nicht als Ausschlußkriterium für die These, solang das sprachliche Vorkommen nicht musterhaft in den Beiträgen eines Unternehmens

\footnotetext{
${ }^{26}$ Sowohl ,super' als auch ,cool' führt der Duden mittlerweile als umgangssprachlich.

${ }^{27}$ Untersuchungen von personal blogs legen nahe, dass die Sprachverwendung nicht in Vorannahme eines breiten Publikums, sondern vielmehr zur Selektion einer ausgewählten, kleinen Leserschaft dient: also genau das Gegenteil (vgl. Puschmann 2013: 94), z. B. Viégas (2005), Brake (2007).
} 
in Erscheinung tritt. Im Gegensatz zu diesen Formen eines nähesprachlichen Ausdrucks finden sich in den Daten nur marginal fachsprachliche Termini. Das unterstreicht den persönlich ungezwungenen Eindruck (vgl. Eroms 2014: 121).

Narrationsspezifische Gestaltungsmittel sind daran ausgerichtet, was der/die jeweilige nicht-journalistisch geschulte Mitarbeiter/in als Erzählung kennt. Hinsichtlich der konkreten narrativen Umsetzung sieht Puschmann (2012) bei ungeübten Blogger/innen eine Nähe zum mündlichen Erzählen, denn

„[w]as [...] tun Blogger, die über keinerlei nennenswerte Schreiberfahrung verfügen? Sie stellen intuitiv einen Bezug zu der Form her, mit der sie bereits vertraut sind: der mündlichen Erzählung.“ (Puschmann 2012: 107)

Die Untersuchung der erhobenen Corporate Blogerzählungen zeigt daneben noch eine weitere Tendenz, die mit den Untersuchungsergebnissen von Schlobinski/Siever (2005: 71) übereinstimmt. Sie sehen in ihren Daten einen Unterschied zwischen einem literarischen Duktus und einer sachbezogenen Orientierung. In Bezug auf die untersuchten Corporate Blogs, auf denen die Mitarbeiter/innen in einem institutionellen Kontext unter Berücksichtigung strategischer Aspekte bloggen, kann m. E. durchaus davon ausgegangen werden, dass sie reflektieren, was sie bisher über das ,Erzählen ' gelernt haben. Genauso ist es möglich, dass begeisterte Leser/innen sich von bereits rezipierter Literatur inspirieren lassen. Auf Basis dieses Weltwissens entstehen Mitarbeitererzählungen, die die Autor/innen gerade durch ihren kreativen Umgang mit Charakteristika des mündlichen und des literarischen Erzählens individualisieren. Dabei erscheinen mündliche Merkmale im Gesamtkontext der uRE eher als narratives Gestaltungsmittel, statt als unreflektiertes Geplauder. Mithilfe der verschiedenen Gestaltungsmittel erhalten die Blogbeiträge einen persönlichen Ausdruck, der der Erzählung den Fingerabdruck eines/r engagierten „Laienblogger/in“ verleiht. Bestimmte narrative Gestaltungsmittel, auf die nicht-journalistische Blogger/innen zurückgreifen, sind in den Daten wiederholt belegt. Welche das betrifft und wie das im Einzelfall aussieht, zeigen die folgenden ausgewählten Beispiele.

Einen authentischen Erlebnisbezug und individuellen Stil offenbaren Blogger/innen deutlich, wenn sie in ihrer Erzählung über einen Auslandsaufenthalt den sprachlichen Code ändern. In dem folgenden ersten Beispiel wechselt die Bloggerin im Vorspann ihrer Erlebniserzählung über ihren beruflichen Aufenthalt in China zwischen Englisch und Chinesisch, um ihre internationale Versiertheit zu betonen. Im abschließenden Ratschlag switcht sie in das Chinesische, was ihre persönliche Verbundenheit mit dem Gastland symbolisiert: 


\section{[Vorspann:]}

Going global? Ni hao ma? Point of no retrun? Integrate, reintegrate or not re-integrable? Ni shi shei? Just Passionate about global liefestyle and the hunger for interculotural fulfilled living? Due, hen hao!!!

[...]

Unser Ratschlag für zukünftige Expats: Mach es! [...]Lass alle Empfindungen zu und sprich darüber! Es ist ein Geschenk! Xiexie! (Daimler, Das Blog, 2014-03-14. Korpusquelle: DAIMLER_062.1_WB)

Eine andere Möglichkeit ist es, mit dem Code-Switching auf eine kulturelle Besonderheit zu verweisen:

Nun, es ist immer Zeit für Kaffee hier in Kroatien. Egal ob um auf der Arbeit wichtige Entscheidungen zu treffen, neue Ideen auszuarbeiten oder in der Pause die Ergebnisse der EM zu diskutieren - alles geht besser mit Kaffee. Die Kaffeepausen zu zelebrieren ist fast schon eine Mentalitätsfrage hier in Kroatien. Zudem war das Bestellen von „bjiela kava“" (so wie Latte Macchiato in Deutschland) das erste was ich ohne Probleme auf Kroatisch hinbekommen habe :D (Dt. Telekom, Blog.Telekom, 201607-12. Korpusquelle: DTKOM_836_WB)

Eine dritte Verwendungsweise dient dazu, die erzählte Situation durch die „reale“ Sprachverwendung stärker in Szene zu setzen:

Als nächstes teilen wir die Kinder in Gruppen nach dem Alter auf und geben ihnen starke Namen. Wir haben Leones (Löwen), Estrellas (Sterne), Halcones (Falken) und noch viel mehr. (Telefonica Dtl., blog.Telefonica, . Korpusquelle: Telef_778_WB)

Das Code-Switchen ist dabei ein bewusst gewähltes Gestaltungsmittel, da es für die Erzählung und zur Authentisierung der Erzähler/innen funktional ist.

Um der uRE eine individuelle Atmosphäre zu verleihen, gibt es ein weiteres narratives Gestaltungsmerkmal, welches nicht-journalistisch geschulte Blogger/innen in den Daten anwenden: das Gedankenzitat. Es ist die Darstellung eines längeren Gedankengangs, der ,durch eine Inquit-Formel in Gestalt eines verbum dicendi oder credendi [...] eingeleitet [wird]“" (Martinez/Scheffel 2007: 60, Hervorhebung i. O.):

- Drei Wochen reichen nie, um den Bewerbungsprozess in Deutschland zu durchlaufen, dachte ich. (Korpusquelle: BOSCH_500_WB) 
- Immer die Gedanken ,Wenn Du dieses Wochenende nichts machst, dann musst Du aber unbedingt nächstes Wochenende etwas arbeiten ....... (Korpusquelle: DAIMLER_055_WB)

- Kurz frage ich mich, ob Helene Fischer gemeinsam mit den freudiglauthalsen Kehlen meiner Kollegen auf der Dezibel-Skala zu einer neuen Kategorie oberhalb des Düsenjets ernannt werden sollte. (Korpusquelle: DAIMLER_090_WB)

Durch das Gedankenzitat geben die Ich-Erzähler/innen einen persönlichen Einblick in das, was sie in einer bestimmten Situation bewegt. In einer uRE, in der der/die Autor/in und das erzählende Ich übereinstimmen, dient das Gedankenzitat dazu, die Erzählung zu individualisieren.

Laienblogger/innen geben ebenfalls einen, jedoch narrativ anders gestalteten, persönlichen Einblick durch atmosphärische Beschreibungen. Die Umsetzung des literarischen Mittels deutet darauf hin, dass die Erzähler/innen an ein Gattungswissen über die Gestaltung eines Spannungsbogens anknüpfen:

„Diese geradezu majestätische Ruhe am Wettkampftag. Die Straßen sind leer gefegt. Keine Autos, die Geschäfte geschlossen... die Vorbereitung ist gelaufen, jetzt zeigt sich, was das Schwitzen gebracht hat, bis der Donnerschlag des Startschuss ertönt...und man nur noch eines vor Augen hat: Das Ziel.“ (Korpusquelle: BOSCH_493_WB)

Bedeutungsschwere Phrasen (majestätische Ruhe, Donnerschlag des Startschusses) in Verbindung mit bildlichen Beschreibungen (Die Straßen sind leer gefegt. Keine Autos, die Geschäfte geschlossen...) und elliptischen Konstruktionen transportieren die Spannung des/der Ich-Erzähler/in. Eine kurze Verzögerung durch die drei Auslassungszeichen und den Satz und man nur noch eines vor Augen hat: münden in die spannungsauflösende Nominalphrase Das Ziel.

Auch in Bezug auf die Anordnung der Ereignisse im Erzähltext zeigen die Autor/innen, dass sie das literarisch narrative Gestaltungsmittel ,Analepse kennen und anwenden. Hierbei handelt es sich um eine Anachronie in der Reihenfolge unterschiedlicher Teile einer Erzählung, bei der ,der zeitliche Abstand zwischen der Zeit, auf die sich der Einschub bezieht, und dem gegenwärtigen Augenblick der Geschichte" (Martinez/Scheffel 2007: 35) divergieren. In dem folgenden Beispiel erzählt eine Mitarbeiterin, wie sie die Teilnahme von Daimler am Christopher Street Day ,ehrenamtlich“ mitgestaltet und erlebt hat. Der vorausgehende Absatz endet mit den Sätzen: Gerade will ich mit einstimmen, da entfernen sich die Gesänge der aus dem gesamten Konzern zusammengewürfelten 
Truppe. Ich nehme nur noch Wortfetzen wahr. Dann folgt die grafisch und begrifflich abgesetzte Analepse (Flashback. Rückblende, Substitution auf Meta-Ebene) (Daimler, Das Blog, 2015-07-29. Korpusquelle: DAIMLER_090_WB):

\author{
Flashback. Rückblende \\ Auch die Zuschauer am Straßenrand, winkend, mitsingend, von uns (oder unseren \\ Gummibärchen?) beglückt, verschwimmen vor meinem inneren Auge zu Schemen. Und \\ urplötzlich versetzen mich meine Gedanken zurück zu einem grauen Novembertag des \\ vergangenen Jahres. Flashback. Rückblende in ein Daimler-Besprechungszimmer in \\ Untertürkheim, das nur geringfügig farbenfroher ist als das Wetter.
}

Durch die Analepse tritt die Erzählerin aus der erzählten Zeit heraus und geht in der Chronologie zurück: Und urplötzlich versetzen mich meine Gedanken zurück zu einem grauen Novembertag des vergangen Jahres. Nun folgen in der Analepse Inhalte von Einzelheiten über bestimmte Herausforderungen während der Organisation. Dann nimmt die Erzählerin den vorherigen Erzählstrang mit den Worten wieder auf: Atemlos! Mein Kollege legt die Hand um meine Schultern und holt mich singend aus meinem Tagtraum zurück auf den Wagen [...]. Die Erzählerin nutzt das literarische Stilmittel zum einen, um sich durch eine detailreiche Schilderung als echte, erlebende Erzählerin auszuweisen. Zum anderen gestaltet sie ihre Erzählung abwechslungsreich und interessant, da die Erzählfolge durch den Einschub für die Rezipient/innen unvorhersehbar wird.

Neben diesen häufig auftretenden narrativen Gestaltungsmitteln verweisen außerdem die Zeitraffung durch elliptische Reihungen (Montag, der 06.10.2014...End of Business Day. Die E-Mail ist vorbereitet. Der Verteiler groß. Der Betreff simpel: AE-CSS BP2015 Presentation. Senden.) und syntaktische Parallelkonstruktionen (Spontan wurde ich angefragt, spontan hatte ich zugestimmt. / Glücklich und stolz, dass sich ihre Arbeitgeber [...]. Glücklich und stolz, dass bei Daimler egal ist [...]) darauf, dass die Erzähler/innen ihr literarisches Weltwissen durchaus in ihre uRE einfließen lassen: Sie gestalten ihren Blogbeitrag mit ihren Mitteln und weisen ihn als den ihren aus.

\title{
9.2.5 Spezifik in Erzählungen journalistisch ausgebildeter Mitarbeiter/innen
}

Erzählungen von journalistisch ausgebildeten Mitarbeiter/innen variieren zwischen einem versierten Umgang mit dem Erzählen und Beiträgen, die denen der 
Laienblogger/innen ähneln. Das liegt zum einen daran, dass die Bezeichnung ,Blogger/in' derzeit keine geschützte Berufsbezeichnung ist. So können neben ausgebildeten Kommunikationsfachleuten ebenso Autodidakt/innen innerhalb der externen Unternehmenskommunikation - zumindest projektbezogen - bloggen. Zum anderen kann in diesen Fällen das strategische Moment der Zielgruppenansprache die Sprache beeinflussen. Nichtsdestotrotz beinhalten die Daten Mitarbeitererzählungen von journalistisch geschulten Erzähler/innen der externen Unternehmenskommunikation, die sich von denen der Laienblogger unterscheiden. Gemeinsam ist beiden Autorengruppen, dass sie ihre Beiträge als beteiligte Ich-Erzähler/innen verfassen. Für journalistisch ausgebildete Erzähler/innen aus der externen Unternehmenskommunikation gilt jedoch, aufgrund des beschriebenen Dilemmas (Abschn. 9.2.3), dass sie sich weniger als authentische Erzähler/innen darstellen möchten, sondern vielmehr ihre Erzähltextinhalte und die darin kommunizierten Einstellungen und Haltungen authentifizieren wollen. Einen besonders hohen Grad an Authentizität besitzt die Redewiedergabe. So verwundert es nicht, dass journalistisch geschulte Erzähler/innen in den Daten dieses narrative Gestaltungsmittel vermehrt und variantenreich anwenden. Besonders anschaulich zeigt sich das Phänomen in uRe über andere Unternehmensmitglieder. Diese uRE basieren auf Interviews und weisen eine Nähe zu Pressetexten auf, die Burger/Luginbühl als Zitatenbericht (2014: 234 ff.) bezeichnen und die nach Schröder (2001: 1722) hinsichtlich ihrer Präsentationsform als Interviewstory weiter differenziert werden können. Bereits die Nähe zu einem journalistischen Textformat zeigt an, dass die Autor/innen auf gewisse Fachkenntnisse zurückgreifen, die ungeübten Laienblogger/innen selten bekannt sind.

In einem Zitatenbericht präsentieren die Verfasser/innen das Interview in Form eines komprimierten Textes, ,in den zahlreiche Zitate aus dem Primärtext mit verschiedenen Formen der Redewiedergabe eingebettet sind" (Burger/Luginbühl 2014: 235). Burger/Luginbühl verweisen allerdings darauf, dass in der linguistischen Literatur bisher für diese Textrealisierung noch keine „stichhaltige Charakterisierung dieser Textsorte“ (ebd. 2014: 237) existiert, weswegen fraglich ist, inwiefern ,,man überhaupt von [einer] konventionalisierten Textsorte sprechen kann“ (ebd. 2014: 237). In diesem Kapitel geht es allerdings nicht darum, dieses Desiderat zu behandeln. Das Ziel dieses Kapitels ist es, erstens ein narratives Gestaltungsmittel aufzuzeigen, das in den Daten von geschulten Blogger/innen besonders häufig verwendet wird. Zweitens kommt hinzu, dass die Autorengruppe dieses Gestaltungsmittel nutzt, um damit die Authentifizierung des Erzählten zu bezwecken. In dem folgenden Kapitel wird es darum gehen, anhand eines Blogbeitrags zu veranschaulichen, wie geschulte Blogger/innen die 
Redewiedergabe zur Gestaltung ihrer uRE einsetzen. Daher stelle ich im Folgenden einen Ausschnitt des ausgewählten Erzähltextes vor und benenne, inwiefern die Realisierung sich den Charakteristika einer ,Interviewstory“ annähert.

\subsubsection{Merkmale der "Interviewstory"}

Den folgenden Erzähltext verfasste eine Mitarbeiterin der Unternehmenskommunikation im Rahmen ihres studienbezogenen Praktikums für den Corporate Blog von Daimler. Den angegebenen Informationen nach studiert die Autorin zu diesem Zeitpunkt Medienkommunikation und Journalismus ${ }^{28}$. Da es sich bei den vorliegenden Erzähltexten um narrative Langformen handelt, greife ich zur Illustration den weitgehend vollständigen Beginn der Blogerzählung heraus. Typografische Merkmale habe ich aus dem Original übernommen und auftretende Klarnamen abgeändert (Daimler, Das Blog, 2015-12-03. Korpusquelle: DAIMLER_069_WB). Die Akteure in der Erzählung sind eine Gruppe von Mitarbeiter/innen um den Protagonisten ,Sven' herum, die die Autorin zum Thema ,Menschen mit Behinderung ' interviewte. Das Interview bettet sie in eine Rahmenerzählung über die Team-Interaktion im beruflichen Alltag ein.

Ganz normal! - Oder?

[...]

„Guten Morgen.“ [Sven] blickt hoch, begrüßt mich, reicht mir die Hand. Ein komisches Gefühl? Nein, warum auch? Er steht zur Begrüßung nicht auf - na und? Er kann es auch nicht. Er sitzt im Rollstuhl. Kein Problem. Weder für mich noch für seine Kollegen.

Das Bearbeiten von Kunden- und -Sponsorenanfragen klappt z. B. ohne Probleme. [Marianne Neugart], ebenfalls in der Erlebniskommunikation tätig stellt klar: „Die Zusammenarbeit verläuft wie mit allen anderen auch - sehr gut nämlich. Man hilft sich gegenseitig, und alles ist ohne Probleme machbar." Martin stimmt ihr zu, beendet das Thema und verweist auf die Mittagspause. Ob ich mitkommen möchte? Klingt gut!

„Lauft schon einmal vor, ich folge auch.“ Habe ich da etwas falsch gesagt? Eigentlich nicht, ich kenne den Weg ja nicht, und auch mein Begleiter meint, er mache sich da keine Gedanken. „Ich sage es auch so“, erklärt mir [Sven]. Aber warum dann dieses unangenehme Gefühl“ - Unsicherheit? Scham? Schlecht gefrühstückt?

„Da komme ich nicht ran.“

${ }^{28}$ Darüber hinaus folgt die Erzählung eines ausgebildeten Autors des Neue Energie-Blog von E.ON innerhalb einer Blogreihe über ausgewählte Mitarbeiter/innen ebenfalls diesem Muster. Das Beispiel ,Hylli` aus der Erzählreihe des Autors findet sich in Abschn. 8.1.2. 
Mittagspause, ein gewohnter Rhythmus: Anstehen, auswählen, bezahlen und essen kein Thema. [Sven] geht es nicht anders. Einzig beim Besteck könnte ich ihm vielleicht helfen. „Da komme ich nicht ran.“ Kein Problem, nur komme ich leider nicht dazu. Ein Kollege von [Sven] ist schneller, kennt die Routine. Besteck reichen und mit dem Fahrstuhl fahren. „Für uns ist es normal geworden und total unproblematisch“, versichert er - für [Sven] übrigens auch.

\section{9 „Keiner sollte darauf bestehen, mir zu helfen.“ 66}

\section{Reserviert - für Menschen mit Behinderung}

[...] Da ist es wieder: Untere Magengegend, schwer einzuordnen, vergleichbar mit einem schlechten Gewissen - aber warum? Zugegeben, für mich ist der Platz nicht reserviert, aber niemand macht eine Bemerkung und auch die anderen scheint es nicht zu stören. Warum nicht? ähnliche Antwort wie zuvor: „Es ist doch völlig normal und außerdem wollen wir ja zusammen essen." Ok, leuchtet ein.

Trotzdem sei es am Anfang schon anders gewesen, ergänzt [August Meier]. „Es war nicht immer klar, wann unsere Hilfe angebracht ist. Es hat aber geholfen, auf [Sven] zuzugehen und ihn einfach zu fragen. Er ist dabei sehr unkompliziert und macht auf sich aufmerksam, wenn er Hilfe braucht.“

„Solche Bedenken sind vollkommen unbegründet“, ergänzt [Sven]. „Man kann mich ruhig darauf ansprechen und seine Hilfe anbieten. Darauf bestehen, helfen zu müssen, sollte man aber nicht - das wäre zu viel des Guten." Das passiert aber selten, denn er kommt ja auch gut zurecht. [...] (Daimler, Das Blog, 2015-12-03. Korpusquelle: DAIMLER_069_WB)

Das zentrale Merkmal der uRE ist, dass die Autorin das geführte Interview in eine geschriebene Form überführt. Das vorliegende Beispiel weicht insofern von dem Merkmal ab, da nicht eine Person interviewt wird, sondern eine Gruppe. Die Wiedergabe der Interviewinhalte inszeniert die Autorin als beiläufige Gespräche im geselligen Beisammensein. Dabei hält sie die dialogische Gesprächsstruktur bei und orientiert sich am Gesprächsverlauf. Insgesamt basiert der Erzähltext auf zusammenfassender sowie auf direkter und indirekter Redewiedergabe. Wie in einem Interview liegt auch in der vorliegenden uRE das Fragerecht und die Gesprächsorganisation bei der Interviewerin. Die Handlungsmöglichkeiten der Interviewten sind auf die Beantwortung der Fragen begrenzt (vgl. auch Holly 1993 zum Fernsehinterview). Metainformationen über das Interview werden im Text selbst nicht gegeben. (vgl. Schröder 2001: 1722 f.) Die Autorin bettet Auszüge aus dem Interview in die Erzählsituation ein. Der Ausdruck ihrer persönlichen Gedanken und Empfindungen rahmt und fokussiert den eigentlichen Gesprächsanlass, den sie am Ende der „Interviewstory“ benennt: Weg ist er [der 


\section{Rollstuhlfahrer] - genau wie meine Bedenken, heute am „Internationalen Tag für Menschen mit Behinderung“.}

Der Auszug verdeutlicht einleitend, dass das „Jonglieren“ mit verschieden Möglichkeiten der Redewiedergabe über das Können eines/einer Laienblogger/in hinausgeht. Dass solche narrativen Vermittlungsvorkommen in den Daten vorkommen, bestätigt, was auch Puschmann (2012) beobachtet:

„In Blogs, die von Nutzern mit Schreiberfahrung (bspw. Wissenschaftler, Journalisten, Autoren) betrieben werden, findet sich oft eine Vielzahl von Merkmalen, welche die Übertragung anderer Genres auf das Blog verdeutlicht. [...] Die Autoren übertragen ihre Vorstellungen bezüglich der Eigenschaften von Genres, in denen sie über einschlägige Erfahrung verfügen, auf Blogs.“ (Puschmann 2012: 106 f.)

In dem nächsten Kapitel stelle ich die verschiedenen Formen der Redewiedergabe anhand des gerade zitierten Beispiels vor und gehe darauf ein, inwiefern die narrative Gestaltungsform für die Authentizität der Erzähltextinhalte gewinnbringend ist.

\subsubsection{Formen der Redewiedergabe}

Die Redewiedergabe findet sich in den untersuchten uRE in zwei Formen. Zum einen handelt es sich um die gesprochene Rede. Sie erscheint in zitierter Rede (hier: direkte Rede), in transponierter Rede (hier: indirekte Rede) und in der erzählten Rede (hier: Erzählbericht). Zum anderen gibt es Passagen, die Gedankenrede wiedergeben. In den untersuchten uRE betrifft das den Inneren Monolog. Als literarisches Stilmittel entspricht er „einer originalen Äußerungsform von Gedanken“ (Zifonun et al. 2011: 1763).

Darüber, inwiefern die Gedankenwiedergabe als Redewiedergabe zu werten ist, gehen die Meinungen auseinander. Gülich (1978) spricht sich dagegen aus. Sie betrachtet insbesondere Einstellungen in Form von Meinungen, Wünschen oder Bedürfnissen nicht als sprachliche Handlungen. Allerdings fügt sie hinzu, dass ihre Betrachtung nicht unproblematisch sei, da beispielsweise in der erlebten Rede durchaus direkte/indirekte Redewiedergabeformen auftreten können (Gülich 1978: 57 f.). Breslauer (1996) betrachtet die innere Rede dagegen „als Kommunikationsakt mit der eigenen Person“ (Leistner 2014: 21) und Zifonun et al. (2011: 1775) sehen die erlebte Rede oder das erlebte Denken als eine spezielle Kontextform an, die charakteristisch für literarische Texte ist. Im Vordergrund steht das Reflektieren einer Person ${ }^{29}$. Die Gedankenwiedergabe in den vorliegenden Texten besteht aus einem Inneren Monolog, den der/die Autor/in in der erzählten Welt mit sich selbst führt. Die Einblicke in ihre Gedankenwelt sind

${ }^{29}$ Weitere Positionen fasst Leistner (2014: 20 f.) zusammen. 
funktional für die Erzählungen, da sie sich scheinbar als Fragen aus dem Interview heraus entwickeln. Entsprechend zielt die jeweilige Gedankenwiedergabe darauf, eine Antwort auf den Inneren Monolog aus der Interviewsituation abzuleiten. Aus diesem Grund liegt eine stilistisch recht eigenwillige Mischung vor, die ich in der Untersuchung als einen Sonderfall der Redewiedergabe betrachte. Aus dem Text selbst wird nicht deutlich, ob die Erzählerin diese Gedanken ausgesprochen hat, doch indizieren die Antworten darauf, dass sie im Interview Thema waren. Zur Verdeutlichung folgen drei Beispiele. Im ersten Beispiel erfolgt die Antwort auf den Inneren Monolog innerhalb der Gedankenwiedergabe. Im zweiten Beispiel folgt die Antwort der Gedankenwiedergabe in Form der indirekten Rede und im dritten Beispiel als direkte Rede (alle Belege entstammen Daimler, Das Blog, 2015-12-03. Korpusquelle: DAIMLER_069_WB):

(Bsp. 1) ,[...] Ein komisches Gefühl? Nein, warum auch Er steht zur Begrüßung nicht auf - na und? Er kann es auch nicht. Er sitzt im Rollstuhl. Kein Problem. Weder für mich, noch für seine Kollegen."

(Bsp. 2) ,„Lauf schon einmal vor, ich folge euch.“ Hab ich da etwas falsch gesagt? eigentlich nicht, ich kenne den Weg ja nicht, und auch mein Begleiter meint, er mache sich da keine Gedanken."

(Bsp. 3) ,Zugegeben, für mich ist der Platz nicht reserviert, aber niemand macht eine Bemerkung und auch die anderen scheint es nicht zu stören. Warum nicht? ähnliche Antwort wie zuvor: „Es ist doch völlig normal und außerdem wollen wir ja zusammen essen.““”

Dass die Autorin durch die Wiedergabe ihres Inneren Monologs eine Reaktion ihrer Interviewpartner/innen im Erzähltext vorbereitet, wird durch die typografisch im Fettdruck hervorgehobenen Fragen deutlich. Sie nutzt die literarischen Stilmittel der Redewiedergabe, um die Botschaft des Interviews zu vermitteln. Durch das stilistische Mittel ,Frage‘ involviert die Erzählerin die Rezipient/innen und kommuniziert im Spiel verschiedener Redewiedergabeformen eine Unvermitteltheit, die wiederum die Authentizität der erzählten Inhalte unterstützt: Zum einen durch die Implikation, der Innere Monolog gebe in der Situation ihre originalen Gedanken wieder, zum anderen durch die zitierten Redewiedergaben, insbesondere die direkte Rede.

Die direkte Redewiedergabe als eine Präsentationsform gesprochener Sprache ist für die (S)D von Unternehmen von großer Bedeutung. Sie wurde bereits in Abschnitt 8.6 im Zusammenhang mit der Identifikation von Mitarbeiter/innen mit ihrem Unternehmen behandelt und sie wird noch in Abschnitt 9.3.3.2 im Rahmen der Ereignisdarstellung von Kompakterzählungen vertieft werden. Die 
direkte/wörtliche Rede muss im Grunde sowohl in der gesprochenen als auch in der geschriebenen Wiedergabe immer markiert sein, damit die Rezipient/innen sie zuordnen können (Leistner 2014: 45). In geschriebenen Texten wird das mithilfe der Interpunktion (Anführungszeichen und/oder Doppelpunkt) und/oder redeanzeigender Verben umgesetzt. Das Potenzial direkter Redewiedergabe im Hinblick auf die Authentifizierung der Erzähltextinhalte liegt darin, dass sie ,,auf eine szenische Vergegenwärtigung der originalen Äußerungssituation ab[zielt]“" (Zifonun 2011: 1755). Dadurch öffnet die Autorin - angelehnt an Brünner (1991) - ein Fenster, in dem die Rezipient/innen die ,Originaläußerung“ miterleben können. Leistner (2014) bringt die Hauptfunktion der direkten Rede in allen wichtigen Aspekten hinsichtlich eines Authentizitätsanspruches auf den Punkt:

„Die Hauptfunktion der DR liegt in der Vergegenwärtigung und Unmittelbarkeit der wiedergegebenen Kommunikationssituation, in der Authentizität und Faktivität des wiedergegebenen Äußerungsinhalts durch die (scheinbare) Ausschaltung des aktuellen Sprechers. Die Verantwortung für den Wiedergabeinhalt liegt beim aktuellen Sprecher.“ (Leistner 2014: 38)

Mit der direkten Rede geht ein gewisser Wahrhaftigkeitsanspruch bezüglich der Aussage einher, da sie suggeriert, den unmittelbaren Standpunkt der sie äußernden Person abzubilden. Der Hinweis in Leistners Zitat ,durch die (scheinbare) Ausschaltung des aktuellen Sprechers“ (ebd.) deutet auf diese Illusion hin. Schließlich rekonstruiert der/die Autor/in die Äußerung (Brünner 1991: 4), passt sie in einen neuen Kontext ein (Leistner 2014: 17 f.) und wählt den gewünschten Auszug der Aussage als Zitat aus (Schröder 2001: 1723). Die direkte Rede findet in dem vorliegenden Text unter narrativen Gesichtspunkten in folgender Funktion Anwendung:

1) Perspektivenwechsel für Bewertungen ${ }^{30}$ : „Die Zusammenarbeit verläuft wie mit allen anderen auch - sehr gut nämlich [...].“ / ,Für uns ist es normal geworden und total unproblematisch. “ / ,Er ist dabei sehr unkompliziert [...]““

2) Dialogische Konzeption als dynamisch, abwechslungsreiches Element: „,Guten Morgen“ / „Lauft schon einmal vor, ich folge euch.“ / ,Ich sage es auch so."

3) Thematische Gliederung der narrativen Struktur anhand von Überschriften: „Da komme ich nicht ran." / „Keiner sollte darauf bestehen, mir zu helfen.“

\footnotetext{
${ }^{30}$ Hier dient das Zitat ebenfalls einer positiven Charakterisierung der Arbeitskolleg/innen, das ist jedoch der Bereich der (S)D.
} 
Die indirekte Rede ist zur Authentifizierung der Erzähltextinhalte gerade aufgrund ihrer Unmittelbarkeit in den Erzähltexten funktional. Mit einer Redewiedergabe wie beispielsweise und auch mein Begleiter meint, er mache sich da keine Gedanken distanziert sich die Erzählerin von der Aussage und belässt sie bei der sich äußernden Person. Sie integriert jedoch ,die Rede in die eigene“ (Martinez/Scheffel 2007: 52) und führt sie so als Beleg für ihre Wahrnehmung an. Hier ist ausschließlich die Charakterisierung des Protagonisten als „unkomplizierte“ Person von Belang, die paradoxerweise gerade durch die Distanz der Autorin glaubhafter werden soll, schließlich ist sie gerade nicht in der Erzählperspektive der Erzählerin verankert. Anwendung findet die indirekte Rede darüber hinaus als narratives Gestaltungsmittel, indem sie zu Zwecken der Beschreibung einer personalen Interaktion genutzt wird. In der vorliegenden uRE setzt die Erzählerin das mittels des Verbs ,mögen`a als Aufforderungswiedergabe um (vgl. Zifonun et al. 2011: 1916): Ob ich mitkommen möchte? Dadurch behält sie die „dialogische“ Konzeption einer Interviewstory bei, ohne die Wechselbeziehung zwischen den Gesprächspartner/innen deskriptiv wiederzugeben.

Das dritte narrative Gestaltungsmittel der gesprochenen Sprache, auf welches die Autorin zurückgreift, ist der Gesprächsbericht. In einem Gesprächsbericht erzählt eine Autorin über einen sprachlichen Akt und gibt den Inhalt allgemein wieder (vgl. Martinez/Scheffel 2007: 62). So kann die Erzählerin die möglicherweise recht umfangreichen Interviewinhalte komprimieren und auf das Wesentliche reduzieren, ohne von der Konzeption der Redewiedergabe abzuweichen. Ein Beispiel hierfür ist die folgende Erzählpassage:

Er spricht vom DTM-Rennen von vor paar Wochen. Interviews betreut, Fachfragen beantwortet, Meisterschaft gefeiert - nur bei der Erlebniswelt konnte er nicht so richtig mithelfen. „Hier ging es hauptsächlich darum, Dinge zu tragen.“ Im Büro $\underline{\text { sieht es dann aber wieder anders aus. Dort bekommt er keine Aufgaben, die er nicht }}$ bewerkstelligen kann.

Mithilfe des Gesprächsberichts rafft die Erzählerin die sehr wahrscheinlich umfangreiche Beschreibung des Protagnisten, indem sie prägnante Punkte in einer Aufzählung herausgreift und schlägt im letzten Satz wieder den Bogen zu ihrem Thema. Dennoch verbleiben die Erzähltextinhalte bei dem Protagonisten, da die Autorin sie nicht erfunden hat, sondern der Ausführung des Protagonisten entnimmt.

Die Ausführungen über Mitarbeitererzählungen auf Corporate Blogs waren darauf ausgerichtet, charakteristische Merkmale des narrativen Vermittlungsvorkommens als narrative Praktiken von Unternehmen herauszustellen. Vor dem 
Hintergrund der Erwartungshaltung an Veröffentlichungen auf Weblogs, zeigt sich, dass auf dem Corporate Blog eines Unternehmens die Mitarbeitererzählungen sich unterscheiden. Dabei wurde herausgearbeitet, dass verschiedene narrative Gestaltungsmittel in den Daten eingesetzt werden, da die Blogger/innen sie nutzen, um dem jeweiligen Dilemma (das mit ihren Erzählungen verbunden ist) entgegenzuwirken. Je nach Vorkenntnissen gestalten die Blogger/innen ihren Beitrag mit ihnen bekannten narrativen Mitteln (literarisch bedingtes Weltwissen, Anlehnung an Mündlichkeit, Adaption spezifischer Genres) und geben ihrer Erzählungen so einen ,individuellen Touch“.

\subsection{Bimodale Kompakterzählungen auf Facebook}

Ich leite das Kapitel mit einem Anwendungsbeispiel ein. Es vermittelt einen ersten Eindruck davon, welches narrative Vermittlungsvorkommen ich unter dem Arbeitsbegriff ,bimodale Kompakterzählung' zusammenfasse. Ich gehe an dieser Stelle nicht erschöpfend auf das Beispiel ein, da es das Beschreibungsobjekt erst einmal veranschaulichen soll. Textuelle und bildliche Spezifika stelle ich in den folgenden Unterkapiteln ausführlich vor. An dieser Stelle geht es darum die (bimodale) Kompakterzählung anhand grundlegender realisierungsbedingter Spezifika darzulegen, die für die Namensgebung ausschlaggebend sind. Eine Einordnung in ein erzähltheoretisches Modell erfolgt am Ende des Kapitels.

Das einleitende Beispiel stammt von dem Energieunternehmen E.ON und wurde auf Facebook veröffentlicht. Während eines Einsatzes auf See mit einem Versorgungsschiff des Unternehmens entdeckten Mitarbeiter/innen an Bord eine Eule, die sich offenkundig nicht in ihrem natürlichen Lebensraum aufhielt. Die Geschichte rund um die Olli genannte Eule veröffentlichte die Unternehmenskommunikation von E.ON auf Facebook und Twitter. Hier folgt exemplarisch der Facebook-Post von E.ON (Abb. 9.8): 
eon

E.ON SE hat 3 neue Fotos hinzugefugt.

29. Oktober 2015 - क्

Schau mir in die Augen, Kleines! Irgendwie hatte sich eine Eule auf eines unserer Versorgungsschiffe vor der schottischen Küste verirt und schipperte 150 Meilen seewarts als blinder Passagier. Reichlich durchgefroren wurde "Olir" von der Crew entdeckt und mit einem herzhaften Hünnchen-Würstchen Früstück aufgepäppelt. Inzwischen befindet sich OIII auf einer Vogelstation in Aberdeen, wo er ausgewildert wird, sobald er sich erholt hat. Unser Tipp: Meide die Küsten, Olli!
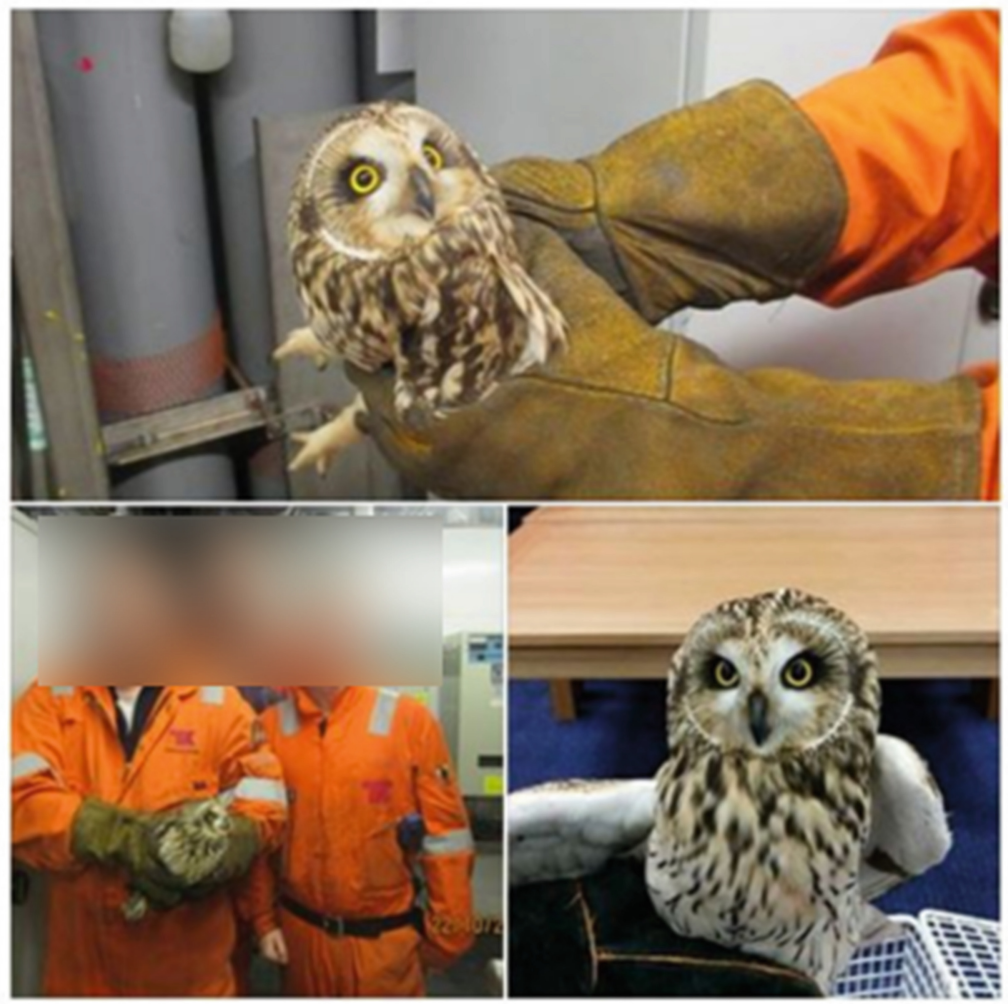

\section{Gefallt mir \\ Kommentieren \\ Teilen}

142

Top-Kommentare *

Abb.9.8 Bsp. für eine bimodale Kompakterzählung: „Eule Olli“ (E.ON, Facebook, 201510-29. Korpusquelle: E.ON_652_FB) 
Das Beispiel illustriert allgemeine realisierungsbedingte Merkmale von Kompakterzählungen:

a. Sie weisen einen geringen textuellen Umfang auf: Ollis Abenteuer wird in fünf Sätzen dargelegt.

b. Der Grad ihrer textuell narrativen Ausarbeitung ist gering: Es werden lediglich Fakten zu Ollis Entdeckung und unmittelbar folgende Handlungen benannt. Mithilfe von Verben (verirrt, schippern, aufpäppeln) und Adjektiven (durchgefroren) entsteht eine narrative Inszenierung, die jedoch nicht vertiefend ausgearbeitet ist.

c. Für gewöhnlich sind bildliche Elemente an Kompakterzählungen gebunden: Die Fotografien einer Eule zusammen mit Personen in Arbeitskleidung legen nahe, dass es sich um Olli und seine Retter handelt. Die beigefügten Bilder illustrieren den Textinhalt. Aufgrund der Beziehung zwischen Text und Bild werden emotionale Assoziationen im textuell vorgegebenen Handlungsrahmen angeregt, die sich auf Olli und seine Wohltäter beziehen.

Die Bezeichnung, Kompakterzählung ' geht darauf zurück, dass es sich um „kleine" Erzählungen handelt (vgl. Small Story nach Bamberg/Georgakopoulou (2008), wie in Abschn. 4.2 beschrieben). Das allein erklärt jedoch nicht, das charakterisierende Kompositum ,Kompakterzählung`. Die charakteristische Kürze der Erzählungen wird von einem Optimierungsgebot bestimmt, welches mit den technischen Rahmenbedingungen verbunden ist. Den Analyseergebnissen nach beinhaltet das Optimierungsgebot vier voneinander abhängige Forderungen:

1. Fasse dich kurz,

2. schreibe das Wichtigste zuerst,

3. benenne nur das Wichtigste und

4. visualisiere sprachlich Aufwendiges.

Das Einhalten des Optimierungsgebotes hat zur Folge, dass die narrativen Strukturelemente (Anfang: Situierung, Mitte: Ereignisrepräsentation, Schluss: Resümee) nicht immer einem grammatischen Gefüge zuordenbar sind ${ }^{31}$. Hinzukommt, dass am Schluss beigefügte Fotografien als weitere semiotische Ressource zu inhaltlichen Verschränkungen zwischen dem Mittel- und Schlussteil der Geschichte führen (hierzu mehr in Abschn. 9.3.4 Text-Bild-Gefüge

${ }^{31}$ Z. B. treten in Ollis Abenteuer situierende und das Ereignis repräsentierende Elemente innerhalb eines Syntagmas auf. 
in bimodalen Kompakterzählungen). In Folge dessen ist die gesamte Erzählung sozusagen verdichtet - eben kompakt. Die Spezifizierung ,bimodal' erhält eine Kompakterzählung, wenn sie zusätzlich mit Fotografien angereichert ist, also wenn zwei Zeichensysteme zusammen wirken $^{32}$. Wie die Autor/innen das Optimierungsgebot in dem narrativen Vermittlungsvorkommen einlösen, verdeutlichen die Analyseergebnisse über die narrative Themenentfaltung. Die folgenden Ausführungen sind dabei an den in der Methode festgelegten Beschreibungsebenen ,Involviertheit der Erzähler/innen', ,narrative Themenentfaltung' sowie der damit einhergehenden ,Erzähltextgestaltung ' orientiert (Abschn. 6.2.2). Einleitend lege ich dar, auf welcher Datengrundlage die Analyseergebnisse beruhen und innerhalb welcher Themenfelder Unternehmen bimodale Kompakterzählungen realisieren (Abschn. 9.3.1).

\subsubsection{Datengrundlage und konstitutive Themenfelder für bimodale Kompakterzählungen}

Insgesamt liegen der Analyse 86 Kompakterzählungen zugrunde (siehe das Balkendiagramm unten). Kompakterzählungen von Unternehmen sind nicht ausschließlich an die sozialen Medien (Facebook, Twitter, Xing, Corporate Blog) gebunden, sondern existieren auch auf der Unternehmenshomepage. In dem bearbeiteten sekundären Subkorpus stammen 73 Kompakterzählungen von Plattformen der sozialen Medien und 20 von U.-Homepages. Neben Schrifttext können diese uRE zusätzlich Fotografien und/oder Bewegtbilder als Gestaltungselemente der Gesamterzählung enthalten. Das ist in den Daten mit Ausnahme von der Internetplattform Xing für alle erhobenen Kompakterzählungen der Fall (Abb. 9.9).

Innerhalb der sozialen Medien sind in dem Korpus die meisten Kompakterzählungen auf Facebook dokumentiert (46 Kompakterzählungen). Aus diesem Grund

\footnotetext{
${ }^{32}$ Ich verwende den Begriff ,Modalität‘ für verschiedene Arten von Zeichen im Sinne ihrer Wahrnehmung (visuell, auditiv u. a.). Treffen mehrere Zeichensysteme zusammen, dann übernehme ich für diese Eigenschaft das Adjektiv ,multimodal'. Häufig findet man in diesem Zusammenhang auch den Terminus ,multimedial'. Beispielsweise spricht Ebert (2000: 215) in Bezug auf Sprach-Bild-Werbeanzeigen von duomedialen Darstellungsformen bzw. Texten. Den Begriff ,Medium` verwende ich für technische Medien wie das Internet, Papier u. a. Zur Vertiefung und für einen Überblick verschiedener Verwendungsweisen systematisiert Habscheid (2009: 96 f.) das ,schwer überschaubare Bedingungsgefüge der Medien“ (ebd.) tabellarisch.
} 


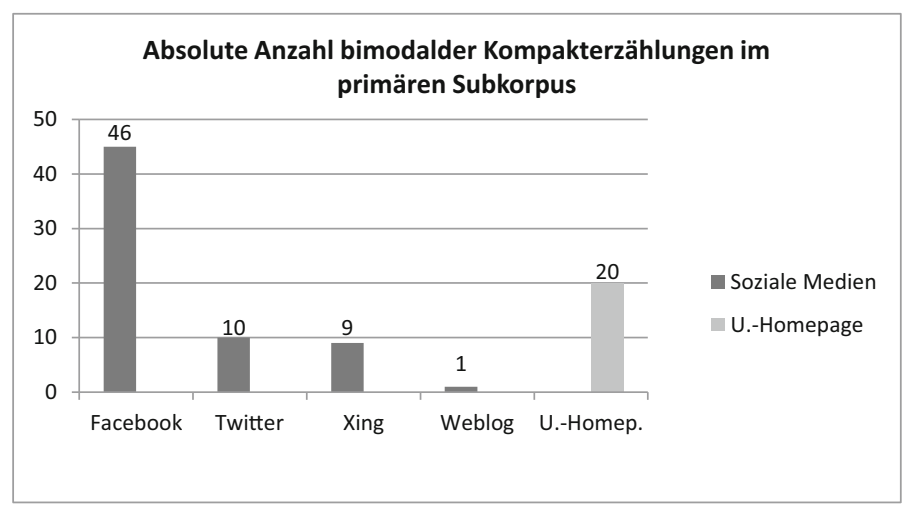

Abb.9.9 Absolute Zahlen der Daten für das narrative Vermittlungsvorkommen ,Kompakterzählung ' im primären Subkorpus $(\mathrm{N}=86)$

gehe ich in dem Kapitel auf spezifische Merkmale des narrativen Vermittlungsvorkommens auf der Internetplattform Facebook ein. Da alle der Daten neben dem Erzähltext über Fotografien verfügen, stehen sie exemplarisch für die ,bimodale Kompakterzählung‘. Hinzukommt, dass die linguistische Forschung das Erzählvorkommen bezogen auf die Unternehmenskommunikation $\mathrm{m}$. W. noch nicht als Forschungsgegenstand untersucht hat. Folglich basiert die sequenzielle Feinanalyse insgesamt auf 46 bimodalen Kompaktzählungen. Vertreten sind die Branchen des Groß- und Lebensmittelhandels (Rewe, $\mathrm{N}=20$ ), der Energie- (EnBW, $\mathrm{N}=$ 10; E.ON, $\mathrm{N}=6$ ) und der Automobilunternehmen (Daimler $\mathrm{N}=10$ ).

Die realisierten Themenfelder lassen nicht nur Rückschlüsse darüber zu, welche Erzählanlässe in dem Korpus für eine Kompakterzählung konstitutiv sind, sondern auch darüber, warum die Erzähltexte sich auf ihre spezifische Weise entfalten. Diese Annahme ist darauf zurückzuführen, dass nicht nur die Internetplattform die Umsetzung, sondern ebenso die Erzählanlässe die Form beeinflussen. Das in Abschnitt 6.2.3 (Themenfeldbestimmung) beschriebene methodische Vorgehen ermöglicht die Einteilung der Erzähltextinhalte von bimodalen Kompakterzählungen (mit wenigen Ausnahmen) in die drei Themenfelder ,Ausbildung/Arbeitsplatz', ,Aktuelle Themen und Öffentlichkeitsarbeit und ,unterhaltende Anekdoten': 
(Bsp.) Umsetzung drei spezifischer Themenfeldern für Kompakterzählung auf Facebook

\begin{tabular}{|c|c|}
\hline $\begin{array}{l}\text { Inhaltlich aufgenommene } \\
\text { Themenfelder }\end{array}$ & Beispiel \\
\hline $\begin{array}{l}\text { 1) Ausbildung / Arbeitsplatz } \\
\text { (Rewe, Facebook, 2016-02-02. } \\
\text { Korpusquelle: REWE_366_FB) }\end{array}$ & $\begin{array}{l}\text { ++ Malermeister und Zentraleinkäufer ++ } \\
\text { Früher, als [Uwe] noch an der Universität Münster } \\
\text { Betriebswirtschaftslehre studierte, hat er zwischendurch immer mal } \\
\text { wieder eine dieser weißen, strapazierfähigen Latzhosen angezogen. In } \\
\text { diesem Dress hat [Uwe] sein Studium finanzieirt. Die Zeit an der Uni ist } \\
\text { lange vorbei und irgendwie hat sich für ihn ein Kreis geschlossen. Denn } \\
\text { [Uwe], der zwischendurch auch eine Malermeisterausbildung an der } \\
\text { Kölner Handwerkskammer absolvierte, macht heute das, was er schon } \\
\text { früher als Jugendlicher und Student getan hat: Er beschättigt sich mit } \\
\text { Farben. Nur seine Arbeitskleidung hat sich geändert. Statt weißer } \\
\text { Latzhose trägt er blauen Anzug. [Huber] ist inzwischen Zentraleinkäufer } \\
\text { für Bodenbeläge, Möbel, Tapeten, Paneele und eben Farben sowie Lacke } \\
\text { bei Toom. }\end{array}$ \\
\hline $\begin{array}{l}\text { 2) Aktuelle Themen und } \\
\text { Öffentlichkeitsarbeit } \\
\text { (E.ON, Facebook, 2016-03-11. } \\
\text { Korpusquelle: E.ON_661_FB) }\end{array}$ & $\begin{array}{l}\text { Kinder \& Störche - das passtl Bald auch im evangelischen } \\
\text { Waldkindergarten Joachimsthal. Inspiriert durch einen Artikel auf unserer } \\
\text { Seite fragte ' [Helge Müller] letzten September an, ob es möglich wäre, in } \\
\text { der Kita seiner Tochter einen Mast mit einem Storchennest aufzustellen. } \\
\text { Gesagt, getan, unsere vogelschutzerfahrenen E.DIS Kollegen halfen und vor } \\
\text { kurzem wurde der Mast aufgestellt. Nun warten Kinder, Eltern und Betreuer } \\
\text { gespannt auf die Ankunft des ersten Storchenpaares. }\end{array}$ \\
\hline $\begin{array}{l}\text { 3) Unterhaltende Anekdoten } \\
\text { (Daimler, Facebook, 2016-03-23. } \\
\text { Korpusquelle: DAIMLER_107_FB) }\end{array}$ & $\begin{array}{l}\text { ++ Strafzettel für flatternde Vorhänge ++ } \\
\text { Aus Strafen können Technologien entstehen. So hat der erste Strafzettel } \\
\text { der Welt den Ausschlag für den innovativen Verkehrszeichen-Assistenten } \\
\text { gegeben. } \\
\text { Daimler bedankt sich bei Alexander Gütermann. Er sorgte } 1895 \text { für } \\
\text { Aufregung, da er mit dem Benz-Motor-Pferd derart durch die badische } \\
\text { Ortschaft Denzlingen raste, dass die Vorhänge zu flattern begannen. } \\
\text { Folge: } 3 \text { Mark Strafe und der erste Strafzettel der Welt. }\end{array}$ \\
\hline
\end{tabular}

Die Themenfelder ,Aktuelle Themen und Öffentlichkeitsarbeit' $(\mathrm{N}=11$; 23 Prozent) sowie ,unterhaltende Anekdoten' $(\mathrm{N}=6 ; 13$ Prozent) werden insgesamt zu 36 Prozent in den untersuchten Kompakterzählungen aufgegriffen. Bezeichnend ist der große Anteil von Inhalten rund um das Themenfeld ,Ausbildung/Arbeitsplatz‘ ( $\mathrm{N}=29 ; 63$ Prozent) ${ }^{\star}$. Die Inhalte handeln von:

- Aktivitäten im Rahmen der Ausbildung: Inhalte über Ausbildungs/Berufsfeldbeschreibungen und/oder Erfahrungen.

- Aktivitäten im Rahmen des Arbeitsplatzes: Inhalte über externes Engagement des Unternehmens und seiner Mitarbeiter/innen, Fort- und Weiterbildung von Mitarbeiter/innen, Unternehmensinitiativen für Mitarbeiter/innen im Sinne der Work-Life-Balance, Arbeitsplatzbeschreibung, beruflicher Werdegang von Mitarbeiter/innen. 
- Außerordentliche Aktivitäten im Zusammenhang mit dem Unternehmen: Inhalte über soziales Engagement, außerberufliche Gemeinschaftsaktivitäten und umweltunterstützende Maßnahmen.

Die Dominanz des Themenfeldes ,Ausbildung/Arbeitsplatz' wird durch die Aussage von Mammen (2015) aus den Kommunikations- und Medienwissenschaften gestützt, der zufolge Facebook Unternehmen eine Plattform zur Mitarbeiteranwerbung bietet, die diese in den hier untersuchten Daten durchaus als solche nutzen. Vor allem in dem Themenfeld ,unterhaltende Anekdoten' sind Unternehmen darauf angewiesen, dass die Verfasser/innen von Kompakterzählungen über entsprechende besondere Erzählanlässe informiert werden. Dagegen sind Kompakterzählungen über ,Ausbildung/Arbeitsplatz` leichter umzusetzen; schließlich können Betroffene das Alltägliche aus ihrer Perspektive problemlos zu einem Erlebnis stilisieren und so die Vorteile/Qualität des Unternehmens im Sinne der (S)D als ,attraktiver Arbeitgeber' herausstreichen (= imagefördernd).

\subsubsection{Involviertheit der Erzähler/innen}

Jede Geschichte benötigt eine/n Erzähler/in. Sie können entweder selbst an dem Geschehen beteiligt gewesen sein (Ich-Erzähler/in: homodiegetische/rErzähler/in) oder sie erzählen von Handlungen, an denen sie selbst nicht teilhatten (ErErzähler/in: heterodiegetische/r Erzähler/in). Für Erzählungen in den sozialen Medien ist das Merkmal ,Authentizität' von großer Bedeutung (vgl. Corporate Blogerzählungen, Abschn. 9.2.3). Im Sinne einer möglichst großen Authentizitätsbestätigung für die uRE wäre ein/e Ich-Erzähler/in zu erwarten. Die Annahme geht darauf zurück, dass mit der Einstellung der Erzählenden zum Erzählgegenstand auch ihre Involviertheit in die Erzählung einhergeht und damit ihr Authentizitätsanspruch steigt. Der steigende Authentizitätsanspruch ist wiederum darauf begründet, dass man einer/m Ich-Erzähler/in, der/die als Augenzeug/in erzählt - insbesondere im Zusammenhang mit expressiven und affektiven Aspekten - intuitiv eine höhere Glaubwürdigkeit einräumt.

Das Korpus zu bimodalen Kompakterzählungen von Unternehmen auf Facebook weist jedoch überwiegend $(\mathrm{N}=42)$ Erzähler/innen in der dritten Person (Er-Erzähler/in) mit einer externen Erzählperspektive auf. In nur sechs Daten spricht ein/e Ich-Erzähler/in in der 1. Person Singular oder mittels des inkludierenden „wir“ (corporate we, Vogel 2011: 138) in der 1. Person Plural. Der Befund ist erwähnenswert, da Facebook als eine Internetplattform der sozialen Medien 
Unternehmen einen weniger formellen Rahmen anbietet als beispielsweise die U.-Homepage. Daher wären Ich-Erzähler/innen, die ihre eigenen Erlebnisse wiedergeben, naheliegend. Ein/e Erzähler/in, der/die beispielsweise die Wir-Form verwendet, zeigt damit nicht nur, Teil des erzählten Kollektivs zu sein, sondern auch, dass er/sie aufgrund ihrer Innensicht stärker von dem Ereignis betroffen ist und somit auch authentischer davon erzählt. Eine uRE in der 3. Person Singular (Herr/Frau ..., Eigenname, Unternehmensname) oder in der 3. Person Plural (110 Azubis, 626 Mitarbeiter, die EnBW-Macher, das Star-Projektteam) ist dagegen näher an der Versprachlichung von berichtendem Erzählen, für das Sachlichkeit und Neutralität kennzeichnend sind (vgl. Narr I, nach Heinemann/Viehweger 1991: 239). Zur Veranschaulichung folgende zwei Beispiele:

(Bsp.) Eine Ich- und eine Er-Erzählung auf Facebook

\begin{tabular}{|c|c|}
\hline $\begin{array}{l}\text { Bsp. 1: Ich-Erzählung (Rewe, Facebook, } \\
\text { 2016-07-15. Korpusquelle: } \\
\text { REWE_352_FB) }\end{array}$ & $\begin{array}{l}\text { Bsp. 2: Er-Erzählung (Daimler-Benz, } \\
\text { Facebook, 2016-07-21. Korpusquelle: } \\
\text { DAIMLER_113_FB) }\end{array}$ \\
\hline 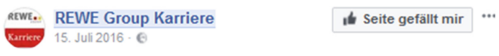 & $\begin{array}{l}\text { Daimler Career hat } 4 \text { neue Fotos hinzugefügt. } \\
\text { 21. Juil - } 6\end{array}$ \\
\hline $\begin{array}{l}\text { ++Berufsorientierungscamp am Stadtgymnasium Köln++ } \\
\text { Letzte Woche waren wir zu Gast an dem Stadtgymnasium in Porz. Im } \\
\text { Rahmen des dort stattfindenden Berufsorientierungscamps boten wir für } \\
\text { alle interessierten Schüler und Schülerinnen neben einer } \\
\text { Unternehmenspräsentation auch ein Bewerbertraining an. Wir führten } \\
\text { einen Bewerbungsmappencheck durch, simulierten ein } \\
\text { Bewerbungsgespräch und gaben den Teilnehmern Tipps und Tricks, wie } \\
\text { sie im Bewerbungsverfahren punkten können. Es hat uns große Freude } \\
\text { gemacht! Wir wünschen euch viel Erfolg beim Bewerben! }\end{array}$ & $\begin{array}{l}\text { Den Fahrzeugkonzepten und Mobilitătstrends der Zukunft auf der Spur. } \\
\text { [Max, Moritz und Udo] studieren alle drei an der Universităt Stuttgart und } \\
\text { bekamen am Dienstag eine exklusive Führung durch die Auto-ldeen- } \\
\text { Schmiede im Mercedes-Benz Werk Sindelfingen, die ,Geburtsstätte* } \\
\text { unserer Forschungs- und Konzeptfahrzeuge. Sie hatten im Mai bei der } \\
\text { 100. Veranstaltung der Vorlesungsreihe ,Technologieführer der } \\
\text { Automobilindustrie stellen sich vor" an der Uni Stuttgart bei unserem } \\
\text { Gewinnspiel mitgemacht und die Plätze für die exklusive Führung } \\
\text { ergattert. } \\
\text { Nun konnten die drei hautnah erleben, was es bedeutet, ein neues } \\
\text { Fahrzeug zu konzipieren - von der Erforschung von Zukunftstrends bis hin } \\
\text { zu Rohbauberechnungen. Außerdem nahmen sie das IAA Concept Car } \\
\text { unter die Lupe und hatten Gelegenheit, im Forschungsfahrzeug F015 } \\
\text { Probe zu sitzen. Das legte nämlich gerade für seinen nächsten Auftritt } \\
\text { einen Boxenstopp in Sindelfingen ein. }\end{array}$ \\
\hline
\end{tabular}

In dem linken Beispiel von Rewe (Facebook, 2016-07-15) erzählt ein/e Mitarbeiter/in mittels des corporate we. Das Pronomen bezieht sich auf das Unternehmen Rewe als Initiator des Ereignisses und die durchführenden Mitarbeiter/innen $^{33}$. Zusätzlich werden die Teilnehmer/innen in dem Abschiedsgruß

${ }^{33}$ Burel (2015: 453) und Gohr (2002: 121) halten bezüglich der Verwendung der „WirFormulierung “ fest, dass Unternehmen sich dadurch als existierende und bekannte Entitäten konstruieren. Die Unternehmensmitglieder müssen so nicht eigens genannt werden, da das Pronomen metonymisch für ihre Namen steht. Auf diese Weise werden die Produzent/innen zum Pluralis Auctoris, der eine einheitliche Unternehmensstimme suggeriert. Das führt zur Konstitution einer Wir-Gemeinschaft (Burel 2015: 454), die aus der Innenperspektive konzipiert und nicht ontologisch gegeben ist. Quantitative Erhebungen der Repräsentationstexte 
Wir wünschen euch viel Erfolg beim Bewerben! adressiert, und wird die Bewertung der Veranstaltenden in dem Exklamativsatz Es hat uns große Freude gemacht! expressiv hervorgehoben. Diese stilistischen Merkmale können zwar auch in uRe in der dritten Person umgesetzt werden, doch erst zusammen mit einerm/r Ich-Erzähler/in wirken sie angemessen. Im Gegensatz zu der expressiven Bewertung in dem Rewe-Beispiel ist die Bewertung, die der/die Erzähler/in in der metaphorischen Wendung nun konnten die drei hautnah erleben anklingen lässt, zurückhaltender, da der/die Erzähler/in selbst nicht Teil des Ereignisses ist und so auch keine eigene affektive Bewertung formulieren kann.

In den Daten wenden Er-Erzähler/innen drei sprachliche Strategien an, durch sie sich in die Nähe und in Beziehung zu den Protagonist/innen stellen, um den Grad ihrer Involviertheit zu erhöhen. So implizieren sie, die Erzähltextinhalte aus erster Hand weiterzugeben:

1) Zum einen geschieht das durch das Einflechten von Zitaten, in denen die am Ereignis Beteiligten oder davon Betroffenen zu Wort kommen (in der Art eines „Miniatur-Features“),

2) zum anderen durch die Verwendung des Possessivpronomens „unser/e“ (unsere Azubis, unser DoktorandInnen). Da dieses Possessivpronomen von dem Personalpronomen, wir ' abgeleitet ist, drückt es nicht nur ein Besitzverhältnis aus, sondern zugleich auch die Zugehörigkeit des/der Erzähler/in zu der Personengruppe, über die er/sie spricht: Die Zugehörigkeit zu demselben Unternehmen ist folglich ausschlaggebend und nicht unbedingt die Beteiligung am Erzählgeschehen.

3) In der dritten Formulierungsalternative verwendet der/die Erzähler/in die Vornamen derer, von denen er/sie erzählt. Das verdeutlicht den persönlichen Bezug zu der Person, auf die er/sie sich in ihrer Geschichte bezieht. Mit dieser persönlichen/vertrauten Anrede stellt sich der/die Erzähler/in in die direkte Nähe der Protagonist/innen und kommuniziert dadurch Teil der erzählten Gemeinschaft zu sein.

Diese drei Optionen, die auch miteinander kombiniert werden (z. B. [Maria], unsere Praktikantin), sollen die Glaubwürdigkeit und die Echtheit der Erzähltextinhalte, die in der dritten Person formuliert sind, stützen und so den erwarteten Authentizitätsanspruch in den sozialen Medien einlösen.

von Unternehmen zeigen, dass das Personalpronomen ,wir“ hochfrequent in der Textsorte „Reprästentationstext“ auftritt (Burel 2015: 451). 


\subsubsection{Narrative Themenentfaltung: inhaltliche Ausarbeitung und textuelle Realisierung}

Eine thematische Entfaltung bedeutet, dass ein Thema mithilfe rhematischer Informationen ${ }^{34}$ weiterentwickelt und fortgeführt wird. Das narrative Vertextungsmusters zeichnet sich dadurch aus, dass auf die Frage „Was ist (mit x) passiert?" (Adamzik 2016: 211) Bezug genommen wird ${ }^{35}$. Nach Brinker et al. (2014: 65) können für eine Erzählung drei narrative Grundkategorien ausgemacht werden:

1) Situierung: grundlegende Angaben zu Ort, Personen und Zeit, die nach Bedarf an unterschiedlichen Stellen in der Erzählung vorkommen können.

2) Repräsentation: Darstellung eines Ereignisses, das ,aus einer oder mehreren Ereignisphasen“ (ebd.: 66) bestehen kann.

3) Resümee: „zusammenfassende Einschätzung vom Erzählzeitpunkt [...] aus“ (ebd. 2014: 66).

Die Autoren (ebd. 2014: 65) gehen dabei - anders als beispielsweise Labov/Waletzky - davon aus, dass die realisierten Sätze bzw. Propositionen nicht unbedingt linear aufeinander folgen müssen, wodurch die tatsächlich realisierte thematische Struktur je nach kommunikativer Funktion verschieden gestaltet werden kann. Diese Annahme ist gerade für die noch in der Entwicklung begriffene uRE ,Kompakterzählung ' ertragreich. Das Modell greift grundlegende inhaltliche

\footnotetext{
${ }^{34}$ Darunter verstehe ich - ausgehend von dem linguistischen Fachdiskurs - Zusatzinformationem zu einem Thema.

${ }^{35}$ Hausendorf et al. (2019) sehen in dem Konzept der narrativen Themenentfaltung u. a. den Zusammenfall von Verknüpfbarkeit und thematischer Zusammengehörigkeit kritisch, da z. B. nicht nur sprachliche Themahinweise, sondern auch ,vertrautheitsabhängige Themaerwartungen aufgebaut werden können“ (ebd. 2019: 208). Daher plädieren sie dafür, nicht von narrativer Themenentfaltung zu sprechen, sondern charakteristische sprachliche Phänomene des Erzählens als narrative Strukturhinweise zu betrachten, denn gerade beim Erzählen sehen sie ,eine Art von Strukturverselbständigung [...]: ein Sprung von der Semantik eines bestimmten Weltbezugs zur Grammatik eines bestimmten globalstrukturellen Verknüpfungstyps“ (ebd. 2017: 209). Da es im Fall der bimodalen Kompakterzählung um eine sehr einfach gehaltene Struktur geht, orientiere ich mich an der narrativen Themenentfaltung nach Brinker et al. (2014).
} 
Aspekte auf, die auch in den Erzähltexten ${ }^{36}$ (Gülich 1976) von Kompakterzählungen, trotz ihrer starken Komprimierung, umgesetzt werden. Entsprechende Merkmale von Kompakterzählungen können daher entlang der drei Grundkategorien erfasst werden. Dabei ist die zuvor vorgenommene Bestimmung der Themenfelder für die narrativ thematische Entwicklung wesentlich. Mithilfe der Fragen, wer, was, wann, wie und warum getan hat/etwas geschehen ist, kann die Entwicklung der Erzählung nachverfolgt werden: Es können Aussagen darüber gemacht werden, welche Informationseinheiten für die jeweilige narrative Grundkategorie (Situierung, Ereignisrepräsentation, Resümee) in Kompakterzählungen konstitutiv bzw. welche unter welchen Umständen verzichtbar sind. Zusätzlich lässt die Platzierung dieser Propositionen in Kompakterzählungen Rückschlüsse auf die Komprimierungsmöglichkeiten (Optimierungsgebot) innerhalb der Erzähltexte zu. Es wird sich zeigen, dass die identifizierten thematischen Kernbereiche und ihre inhaltliche Umsetzung (Abfolge und Anordnung der Propositionen) einen Einfluss auf charakteristische Merkmale von Kompakterzählungen haben.

In diesem Kapitel gehe ich nun ausschließlich auf die verschriftlichte narrative Themenentfaltung ein. Dabei orientiere ich mich zur Beschreibung der Charakteristika an den drei narrativen Grundkategorien von Brinker et al. (2014). Hierfür lege ich die narrative inhaltliche Ausarbeitung mithilfe der konkreten Textrealisierung dar. So verbinde ich das ,Was' der Erzählung mit dem ,Wie‘.

\subsubsection{Optionen der Situierung}

Grundsätzlich sind auf Weblogs und in den sozialen Medien neben den eigentlichen Kompakterzählungen Informationen zum a) Datum der Veröffentlichung und b) dem Unternehmen, für welches die Kompakterzählung verfasst wurde, dokumentiert. Zur Veranschaulichung ist unten noch einmal der Facebook-Post zu Eule Olli abgebildet. Die Pfeile verweisen auf die entsprechenden Zusatzinformationen oberhalb der bimodalen Kompakterzählung (Abb. 9.10):

36 ,[D]as was durch die Sprechhandlung schriftlich oder mündlich geäußert wird“ (Gülich 1976: 226) 


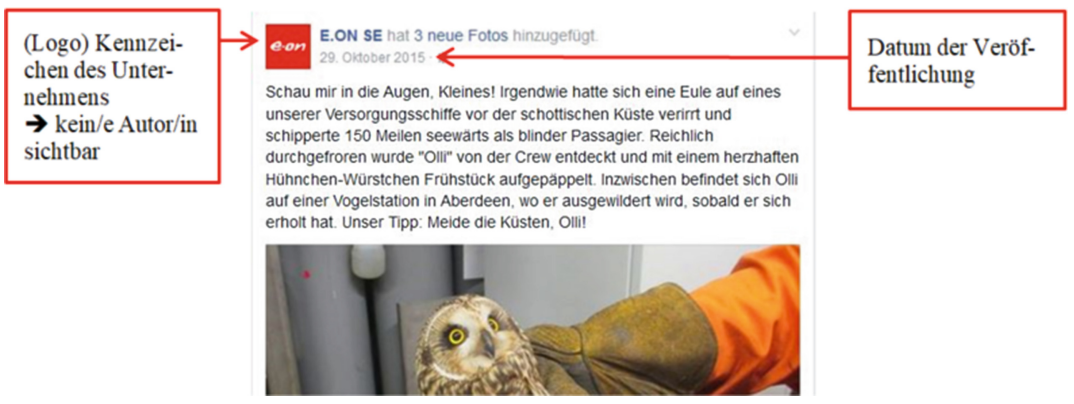

Abb.9.10 Zusatzinformationen im „Kopf“ eines Facebook-Posts (E.ON, Facebook, 201510-29. Korpusquelle: E.ON_652_FB)

Durch die einheitliche „Kopf-“ Gestaltung sind die folgenden Erzähltextinhalte direkt an das jeweilige Unternehmen gekoppelt. Das ist wichtig, da nicht immer unternehmensspezifische Erkennungsmerkmale in den Erzähltexten aufgegriffen werden (wie auch im Fall des Posts zu Eule Olli). Als weitere Information kann eine Rubrikangabe (z. B. ,Karriere') hinzukommen.

Den eigentlichen Erzählbeginn der Kompakterzählung signalisiert in der Überzahl $(\mathrm{N}=32$ von 46) eine optisch mittels typografischer Zeichen (meistens eine doppelte Plus-Zeichenfolge ,++“) oder durch einen Absatz abgehobene Überschrift (Anfangsmarkierung; Sandig 2006: 177). Sie fokussiert die Leser/innen auf das Thema. Die Überschrift ist somit ein erster thematischer Hinweise. Wird über einen Gegenstand oder Sachverhalt etwas verbalisiert, so sagt man damit auch etwas über ein Thema aus und verankert es so im aktuellen Aufmerksamkeitsbereich der Rezipient/innen (Hoffmann 2000: 350): Es handelt sich bei diesen Überschriften also um eine strukturelle Verdichtung mit Themahinweis. Sprachlich treten solche überschriftinduzierten Anfangsmarkierungen in Form von Nominal-/Präpositionalphrasen oder in Form von Struktur-Ellipsen (Zifonun et al. 1997) auf, wie folgende Beispiele illustrieren:

- Nominalphrase

$\bigcirc++$ Digitale Innovationen und erfinderische Mitarbeiter: Der DigitalLife Day 2016++(Daimler-Benz, Facebook, 2016-05-28. Korpusquelle: DAIMLER_109_FB)

○ ++Entspannter Quereinsteiger++(Rewe, Facebook, 2015-06-25. Korpusquelle: REWE_371_FB) 
○ ++Azubi-Grillfest++(Rewe, Facebook, 2016-06-08. Korpusquelle: REWE_360_FB)

- Präpositionalphrase

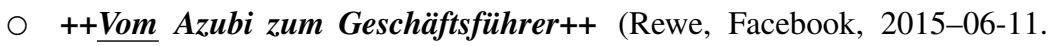
Korpusquelle: REWE_363_FB)

○ Als Praktikantin bei der Nachwuchsförderung++ (Rewe, Facebook, 201606-24. Korpusquelle: REWE_368_FB)

○ Hochspannung am Storchennest (EnBW, Facebook, 2014-07-02. Korpusquelle: EnBW_729_FB)

- $\underline{\text { Struktur-Ellipse }}$

○ Duales Studium - Beste Karriereaussichten und Auslandsmöglichkeiten [sind] inklusive! $\rightarrow$ Tilgung des Kopulaverbs (Rewe, Facebook, 2014-0708 / Korpusquelle: REWE_354_FB)

$\bigcirc++$ Bergmann-Brauerei unter REWE Markt [entdeckt]++ $\rightarrow$ Tilgung des Vollverbs (Rewe, Facebook, 2016-03-17. Korpusquelle: REWE_351_FB)

$\bigcirc$ Unsere Azubis [arbeiten/sind unterwegs] in sozialer Mission $\rightarrow$ Tilgung des Vollverbs (EnBW, Facebook, 2014-12-02. Korpusquelle: EnBW_710_FB)

Diese drei strukturverdichtenden Formen sind charakteristisch für Texte, die extremen Ökonomieanforderungen unterliegen - wie beispielsweise das Telegramm oder die Schlagzeile. Die archivierten Struktur-Ellipsen werden vorrangig durch das Weglassen des finiten Verbs (Finitum-Ellipse, Zifonun 1997: 343) oder des Kopulaverbs realisiert.

Ein weiteres Anfangsmerkmal, welches sowohl in einer Überschrift als auch am Beginn der eigentlichen Kompakterzählung auftritt, ist die generalisierende Sentenz. Dabei wird die Ausgangslage so beschrieben, dass die Referenz von einer speziellen Person auf viele/mehrere mögliche Personen übertragen wird, z. B.:

- Jeder mag den Kerl mit dem roten Schnabel (E.ON, Facebook, 2015-04-14. Korpusquelle: E.ON_662_FB)

- Kinder \& Störche - das passt! (E.ON, Facebook, 2015-04-14. Korpusquelle: E.ON_661_FB) 
- Laut einer Statistik verliebt sich jeder Fünfte am Arbeitsplatz (Daimler-Benz, Facebook, 2016-08-06. Korpusquelle: DAIMLER_121_FB)

- Wer forscht, der profitiert von bereichsübergreifendem Wissenstransfer (Daimler-Benz, Facebook, 2016-07-12. Korpusquelle: DAIMLER_110_FB)

Dieses Anfangsmerkmal, das die narrative Entwicklung nach dem Muster, vom Allgemeinen zum Speziellen' strukturiert, hat die Funktion, die Kompakterzählung mit einer impliziten Begründung einzuleiten. Sinngemäß kann in diesen Fällen der Beginn des folgenden Satzanschlusses mit Kausaladverbien wie deshalb, deswegen, daher oder einer kausalen Phrasen wie aus diesem Grunde ergänzt werden. Dies belegt das folgende Beispiel von Daimler:

„Wer forscht, der profitiert von bereichsübergreifendem Wissenstransfer. Deswegen fand letzte Woche der Daimler Doktorandenmarktplatz statt - ..." (Daimler-Benz, Facebook, 2016-07-12. Korpusquelle: DAIMLER_110_FB)

Ist keine Überschrift vorhanden, beginnen Kompakterzählungen auch mit Exklamativsätzen, denen der weitere Fließtext direkt folgt. Ist das der Fall, dann wird dabei gleichzeitig der „Höhepunkt“ der Kompakterzählung vorweggenommen, wie die folgenden Beispiele illustrieren:

- Happy End für ein verliebtes Falkenpärchen! (E.ON, Facebook, 2015-03-17. Korpusquelle: E.ON_663_FB)

- Azubis an die Macht! (Rewe, Facebook, 2015-09-03. Korpusquelle: REWE_349)

- Eine kleine Erfolgsstory mit gutem Ende für den Klebitz! (EnBW, Facebook, 2014-08-21. Korpusquelle: EnBW_709_FB)

Diese Textgliederung erinnert an das Aufbauprinzip von Nachrichten nach dem Modell der umgekehrten Pyramide (inverted pyramid, Weischenberg 2001: 79): Das Modell besagt, dass das Wichtigste am Anfang steht, während gegen Ende die Bedeutung der Information sukzessive abnimmt. So kann der „Höhepunkt“ an den Beginn der Erzählung verschoben werden, was sich in zweierlei Hinsicht auswirkt: Einerseits weckt dies das Interesse nach Zusatzinformationen, es animiert also zum Weiterlesen, und jene, die nicht weiterlesen werden, rezipieren andererseits dennoch die Grundinformation. Eine Grundinformation, die für die imagefördernde (S)D des Unternehmens essenziell ist und so auch unmotivierte 
Leser/innen erreicht ${ }^{37}$. Bezogen auf die narrative Themenentfaltung gehören sowohl die Überschrift, der Exklamativsatz, die generalisierende Sentenz als auch der vorangestellte „Höhepunkt“ bereits zur Situierung, die in Kompakterzählungen auf das Nötigste beschränkt ist und innerhalb nur eines Satzes mit der Ereignisrepräsentation verbunden sein kann.

Inhaltlich kann die Situierung nach Brinker et al. (2014: 65 f.) unterschiedliche Informationen beinhalten. Das ist abhängig vom Informationsstand der Leser/innen. Unentbehrlich für die erste Situierung ${ }^{38}$ in den vorliegenden Kompakterzählungen sind die Protagonist/innen und Akteure (diesbezügliche Propositionen beziehen sich auf die Fragen: Wer handelt? Wen betrifft die Handlung?) sowie das Thema (Was ist geschehen? Von wem/was handelt die Erzählung?), das in verschiedenen Ausführungen als Anfangsmerkmal vorangestellt werden kann. Wogegen die Frage nach dem Ort abhängig vom Thema auch im Rahmen der Repräsentation beantwortet werden oder unbeantwortet bleiben kann. Das ist der Fall, wenn in der Erzählung das Ereignis an sich für die (S)D im Vordergrund steht wie zu Beginn des folgenden Beispiels:

„Gestern fand zum zweiten Mal unser Daimler Doktorandentag statt. Unter dem Motto „Gestalte Deine Zukunft: Werde Teil von Daimlers Visionen und betrete gemeinsam mit uns neue Pfade“", bot die Veranstaltung 130 teilnehmenden DoktorandInnen vielfältige Highlights [...]“ (Daimler-Benz, Facebook, 2015-11-20. Korpusquelle: DAIMLER_111_FB)

In dem Beispiel fokussiert die Kompakterzählung die Initiative von Daimler (unser Daimler Doktorandentag), durch die der Konzern seine Mitarbeiter/innen miteinander vernetzt, um so Synergieeffekte zwischen den Promotionsprojekten zu generieren - ein Element der imagefördernden (S)D als attraktiver Arbeitgeber. Der explizite Ort des Geschehens ist daher zweitrangig. Im Gegensatz dazu ist im nächsten Beispiel der Ort funktional für die imagefördernde (S)D:

\footnotetext{
${ }^{37}$ Aspekte der imagefördernden (S)D in den obigen Beispielen sind: a) Es wird ein Bezug zwischen der Natur und dem Unternehmen hergestellt, insofern sich für die Natur etwas durch das Unternehmen verbessert hat (= Grundtyp 3 der (S)D), und b) es wird den Auszubildenden außerordentliche Verantwortung übertragen, um sie zu fordern und zu fördern (= Grundtyp 4 der (S)D).

${ }^{38}$ Nach Brinker et al. (2014: 65 f.) können situierende Elemente außerdem auch diskontinuierlich, an verschiedenen Textstellen auftreten oder gänzlich fehlen.
} 
„Florian, Philipp und Tilo studieren alle drei an der Universität Stuttgart und bekamen am Dienstag eine exklusive Führung durch die Auto-Ideen-Schmiede im MercedesBenz Werk Sindelfingen, die „Geburtsstätte“ unserer Forschungs- und Konzeptfahrzeuge [...]“" (Daimler-Benz, Facebook, 2016-07-21. DAIMLER_113_FB)

Der Zusammenhang zwischen der Ortsbenennung Werk Sindelfingen und der (S)D des Unternehmens entsteht durch die auf den Ort bezogenen Metaphern Auto-Ideen-Schmiede und Geburtsstätte unserer Forschungs- und Konzeptfahrzeuge. So ist die Ortsbenennung der Anker für die metaphorischen Hochwertwörter (Janich 2010) Ideen-Schmiede und Geburtsstätte, die das MercedesBenz Werk in Sindelfingen ,aufgrund ihrer sehr positiven Inhaltsseite“ (Janich 2010: 169) als außergewöhnlich und innovativ aufwerten. Auch die Frage, wann das Ereignis stattfand (Zeitpunkt), ist in Fällen, in denen das Ereignis an sich als zentrales Element der (S)D fungiert, sekundär:

„Laut einer Statistik verliebt sich jeder Fünfte am Arbeitsplatz. So geschehen bei Stephanie und Frank, die beide im Mercedes-Benz Werk Sindelfingen, in der Produktion der S-Klasse Bau 46, arbeiten [...]“ (Daimler-Benz, 2016-08-06. Korpusquelle: DAIMLER_121_FB)

Das Ereignis besteht darin, dass sich zwei Angestellte am Arbeitsplatz verlieben und, wie das im weiteren Verlauf deutlich wird, mit dem vom Unternehmen zur Verfügung gestellten Mercedes S-Klasse zu ihrer Vermählung fahren. Der Zeitpunkt der Hochzeit ist in diesem Fall von geringer Bedeutung. Im Mittelpunkt steht das Ereignis des Verliebens, verbunden mit dem, was das Unternehmen seinen Mitarbeiter/innen ermöglicht. Im Sinne des Optimierungsgebots sind demnach Aussagen zu konkreten Zeitpunkten innerhalb des Erzähltextes nicht wichtig. Wer dennoch an einem Zeitpunkt interessiert ist, kann sich diesen anhand der Post-Veröffentlichung oberhalb der Kompakterzählung ungefähr erschließen.

Grundsätzlich werden für die Situierung in den untersuchten Kompakterzählungen maximal drei Sätze mit unterschiedlicher Komplexität aufgewendet. Die Länge ist teilweise davon abhängig, inwieweit eine Überschrift bereits die Frage nach dem Ereignis ausreichend beantwortet. In diesen Fällen kann der folgende Erzähltext reduziert werden:

Eine kleine Erfolgsstory mit gutem Ende für den Kiebitz!

In Tannheim wurden vor rund einem Jahr zwei künstliche Inseln mit Stausee der Iller gebaut - eine Maßnahme der EnBW im Rahmen der Instandsetzung des Mooshauser Wehrs. [...]“ (EnBW, Facebook, 2014-08-2. Korpusquelle: EnBW_709_FB) 
Die Information in der Überschrift (fett hervorgehoben) ist für das Verständnis dieser Kompakterzählung grundlegend. Ohne sie würden wir nicht erfahren, was das Erzählenswerte daran ist, dass die EnBW sich für die Instandsetzung des Mooshauser Wehrs einsetzt (= Artenschutz für eine Vogelart). Entsprechend kurz fällt die textuelle Einführung im Anschluss daran aus: Ein Satz mit Rechtsherausstellung (Schwitalla 2006: 115). Solche situierenden Sätze können mitunter komplex sein, je nachdem wie viele Informationen in einem Satz untergebracht werden. Mögliche Zusatzinformationen sind beispielsweise Projekt- oder Veranstaltungsbenennungen:

„Das REWE Group Star-Projektteam ,die drei lustigen zwei“ haben sich mit ihrem Projekt „Anderen Menschen mit Nachhaltigkeit eine Freude machen“ vorgenommen, aus Restholz Gesellschaftsspiele anzufertigen und diese dann in einer Tagespflege zu spielen.“ (Rewe, Facebook, 2014-11-10. Korpusquelle: REWE_370_FB)

Dabei handelt es sich um „sprechende“ Benennungen ${ }^{39}$ wie im obigen Beispiel „,die drei lustigen zwei“ und ,[a]nderen Menschen mit Nachhaltigkeit eine Freude machen". Sie kommunizieren Merkmale (hier soziales Engagement, fröhliche und engagierte Mitarbeiter/innen), die unverzichtbar für eine unternehmensgebundene Kompakterzählung zur imagefördernden (S)D sind und unterliegen aus diesem Grund nicht dem Optimierungsgebot des Realisierungstypen.

\subsubsection{Charakteristische Merkmale der Ereignisrepräsentation}

Dieses Unterkapitel behandelt Charakteristika von Kompakterzählungen hinsichtlich der Ereignisrepräsentation. Die Repräsentation eines Ereignisses konstituiert sich aus einer oder mehreren Ereignisphasen (Brinker et al. 2014: 66), die im Thema der Erzählung verankert sind. Eine Ereignisphase beinhaltet nach Brinker et al. (2014) eine narrative Ereignis- oder Handlungssequenz, die eine Komplikation und deren Auflösung abbildet.

Ein typisches Merkmal für bimodale Kompakterzählungen ist, dass sie keine prototypischen Höhepunkterzählungen sind. Zurückzuführen ist das auf die Dominanz der Erzählanlässe im Themenfeld ,Ausbildung und Arbeitsplatz‘. In

\footnotetext{
${ }^{39}$ Weitere Beispiele: „Gestern fand zum zweiten Mal unser Daimler Doktorandentag statt. Unter dem Motte „Gestalte Deine Zukunft: Werde Teil von Daimlers Visionen und betrete gemeinsam mit uns neue Pfade“, bot die Veranstaltung..." (Daimler, Facebook, 2015-1120. Korpusquelle: DAIMLER_111_FB) oder „Als Linda in den letzten Zügen ihres Bachelorstudiums der Kommunikationswissenschaft war, las sie auf Facebook von einer unserer „WhatsApp @ Daimler“-Aktionen und nahm daran teil.“ (Daimler, Facebook, 2016-02-25. Korpusquelle: DAIMLER_114_FB)
} 
diesem Bereich ist das thematisieren einer Komplikation für die $(\mathrm{S}) \mathrm{D}$ kontraproduktiv. Ist dennoch eine vorhanden, dann wird sie lediglich erwähnt, aber nicht narrativ ausgearbeitet. Wird in einer Kompakterzählung eine Komplikation genannt, ist bezeichnend, dass die Komplikation außerhalb des Einflussbereichs der Unternehmen verortet ist: Solche Komplikationen beziehen sich auf Umwelteinflüsse oder eigentlich ungünstige Handlungen von Personen, die retrospektiv für das Unternehmen positiv umgedeutet werden. Das folgende Beispiel illustriert eine Komplikation (fett hervorgehoben), die durch schlechte Wetterverhältnisse bedingt ist:

„Happy End für ein verliebtes Falkenpärchen! Ein Sturm zerstörte letztes Jahr den Wanderfalkenbrutplatz am Kühlturm des E.ON Kraftwerks Heyden in Petershagen. Unsere Azubis fertigten einen neuen Brutkasten, der nun in 110 Meter Höhe angebracht wurde - gerade noch rechtzeitig zur Balz- und Brutzeit. Wir hoffen auf baldigen Falkennachwuchs!“ (E.ON, Facebook, 2015-03-17. Korpusquelle: E.ON_663_FB)

In diesem Beispiel löst ein Unwetter, das den Nistplatz von Wanderfalken an einem Kühlturm des E.ON Kraftwerkes zerstört (Ein Sturm zerstörte [...] den Wanderfalkenbrutplatz), die Komplikation aus. Wie sich das zugetragen hat, wie sich die Not der Wanderfalken ablesen lässt, wie der zerstörte Nistplatz entdeckt wurde u. a. - all diese Aspekte könnten narrativ ausgeführt werden: Das geschieht in einer Kompakterzählung aber gerade nicht. Eine derartige Komplikation ist unverfänglich, da Naturgewalten sie verursachen. Ebenso verhält es sich mit Komplikationen, die sich dem Handlungsbereich von Unternehmen entziehen, wie das folgende Beispiel vorführt (die Komplikation ist hervorgehoben):

„++ Strafzettel für flatternde Vorhänge ++

Aus Strafen können Technologien entstehen. So hat der erste Strafzettel der Welt den Ausschlag für den innovativen Verkehrszeichen-Assistenten gegeben.

Daimler bedankt sich bei [Joseph Meier]. Er sorgte 1985 für Aufregung, da er mit dem Benz-Motor-Pferd derart durch die badische Ortschaft Denzlingen raste, dass die Vorhänge zu flattern begangen. Folge: 3 Mark Strafe und der erste Strafzettel der Welt.“ (Daimler, Facebook, 2016-05-23. Korpusquelle: DAIMLER_107_FB)

Diese Komplikationen sind aufgrund ihres Ursprungs außerhalb der Wirkungssphäre des Unternehmens unverfänglich und bieten keine Angriffsfläche, die sich für das Image des Unternehmens nachteilig auswirken könnte. Das Erzählenswerte daran ist, dass Unternehmen veranschaulichen, wie ihre Mitarbeiter/innen mit bestimmten Situationen umgehen und wie sie etwas für die Gesellschaft 
und/oder die Umwelt tun. Zugleich ist das ein weiteres Indiz für die deutliche Präsenz der imagefördernden (S)D von Unternehmen in Kompakterzählungen.

Eine Folge des Optimierungsgebots ist es, die Ereignisse chronologisch aufzuzählen wie in dem untenstehenden Ausschnitt von Rewe (Aufzählungen sind fett hervorgehoben):

„Der Tag begann mit interessanten Vorträgen über unseren Einkauf, unsere Eigenmarkenentwicklung, sowie über die Einkaufsbüros der REWE Far East in Asien. Nach dem Mittagessen ging es mit dem Bus nach Bergisch-Gladbach, wo wir die Produktion der Firma K-FEE besichtigen durften.“ (Rewe, Facebook, 2016-05-26. Korpusquelle: REWE_376_FB)

In dem Beispiel werden die zwei Ereignisse Vorträge und Besichtigung der Produktion gemäß ihrer zeitlichen Abfolge benannt und mittels des Vollverbs beginnen (Anfangsmerkmal, Gülich 1976: 243) und dem Temporaladverb nachdem (Nachfolgemerkmal, Gülich 1976: 243) verbunden, die als Episodenmerkmale (Gülich 1976 234) den Erzähltext retrospektiv in seinem zeitlichen Verlauf strukturieren. Auch in diesem Beispiel zeigt sich die gängige Praxis, Komplikation auszusparen. Das soll im Umkehrschluss jedoch nicht bedeuten, dass Kompakterzählungen keinen Höhepunkt haben. Er wird lediglich verschoben! Und zwar im unmarkierten Fall auf Evaluationen und Bilder/Bilderserien.

Ist die Ereignisrepräsentation narrativ stärker ausgebaut, findet sich darin das Wiederaufnahmeprinzip, welches das Textthema indiziert. Aufgrund des Optimierungsgebots fallen dann jedoch die Strukturelemente ,Situierung ' und ,Resümee a) kurz aus oder b) werden satzübergreifend realisiert. Hierzu noch einmal zu ,Eule Olli` auf dem Versorgungsschiff von E.ON:

\footnotetext{
„[Anfangsmarkierung $\rightarrow$ ] Schau mir in die Augen, Kleines! [Situierung $\rightarrow$ ] Irgendwie hatte sich eine Eule auf eines unserer Versorgungsschiffe vor der schottischen Küste verirrt [Ereignisrepräsentation $\rightarrow$ ] und schipperte 150 Meilen seewärts als blinder Passagier. Reichlich durchgefroren wurde „Olli“ von der Crew entdeckt und mit einem herzhaften Hühnchen-Würstchen Frühstück aufgepäppelt. Inzwischen befindet sich Olli auf einer Vogelstation in Aberdeen, wo er ausgewildert wird, sobald er sich erholt hat. [Fazit $\rightarrow$ ] Unser Tipp: Meide die Küste Olli!“ (E.ON, Facebook, 2015-10-29. Korpusquelle: E.ON_652_FB)
}

Das Thema der Erzählung ist eine ,verirrte Eule auf dem Versorgungsschiff‘. Es wird durch die Personifizierung des Vogels (Namensgebung Olli) gesetzt und dann in jedem Folgesatz wiederaufgenommen. Situierung und Repräsentation fallen bereits im zweiten Satz direkt nach der Themenbenennung zusammen und überlappen sich innerhalb einer grammatischen Einheit. Den Schluss (hier ein 
Fazit) reduziert der/die Verfasser/in am Ende auf die Sprechhandlung ,Raten “ und bindet ihn durch die humorvolle Konnotation an die ebenfalls humorvolle Erzähleröffnung Schau mir in die Augen, Kleines! zurück. In diesem Beispiel ist die Repräsentation für eine Kompakterzählung „ungewöhnlich“ ausgebaut: Das Ereignis wird durch die Handlungen der Beteiligten sowie die Zustandsbeschreibung von Eule Olli narrativ gestaltet. Entsprechend muss der/die Autor/in die textuelle Komprimierung an anderer Stelle, nämlich in der Situierung und am Ende, einlösen ${ }^{40}$ : Beide fallen entsprechend knapp aus.

Eine andere Variante der Ereignisrepräsentation von Kompakterzählungen legt nahe, dass sich die Verfasser/innen dabei an bereits bestehenden journalistischen Genres orientieren: In diesem Fall handelt es sich um das Feature. Ein Feature gehört zu den sogenannten weichen Nachrichtenformen ${ }^{41}$. Bezeichnend für diese ist, dass sie in unterschiedlichem Maß neben dem Schildern und Beobachten, auf einen erzählenden Duktus zurückgreifen. Die Funktion eines Features besteht darin,

„die journalistischen Darstellungsmöglichkeiten [...] zu erweitern: mit Informationen auch zu unterhalten, hinter die Fakten schauen zu lassen, zu erklären und einzuordnen, Interpretations- und Orientierungshilfen zu geben, zu thematisieren und zu generalisieren.“ (Weischenberg 2001: 177, Hervorhebung U.A.)

Speziell im Feature werden allgemeine Sachverhalte an Einzelfällen, also persönlichen Geschichten, veranschaulicht (Interview mit G. Fischer im Bayerischen Rundfunk, 2017-10-23) ${ }^{42}$. Hierfür wenden die Autor/innen stilistische Mittel

40 Auch hier ist die „Komplikation“,eine Eule außerhalb ihres artgerechten Lebensraums“ für das Unternehmen unverfänglich und wird zugunsten der (S)D der Handelnden als Tierfreunde, die das Unternehmen vertreten, narrativ inszeniert.

${ }^{41}$ Die Unterscheidung von ,weichen“ und „harten“ Nachrichten ist in erster Linie inhaltlich motiviert. Während „harte“ Nachrichten Themen aus der Politik und Wirtschaft behandeln, konzentrieren sich ,weiche“ Nachrichten u. a. auf den Bereich ,human interest“ (Lüger 1995: 103, nach Burger/Luginbühl 2014: 225).

${ }^{42}$ In der Reportage verhält es sich nach Fischer dagegen umgekehrt. Hier steht der Einzelfall nicht exemplarisch für etwas Allgemeines, sondern als das eigentliche Thema für sich. Vor dem Hintergrund, dass die externe Unternehmenskommunikation auf Erzählungen zurückgreift, um grundsätzliche Werte, Ansichten oder Einstellungen (z. B. in Form von Arbeitsbedingungen, sozialem Engagement u. a.) des Unternehmens nach außen zu kommunizieren, stehen die Akteure mit ihren Geschichten exemplarisch für den entsprechenden Themenbereich. Aus diesem Grund fiel die Entscheidung, Kompakterzählungen als eine Art „Miniatur-Features“ zu betrachten. Zum kritischen Diskurs über Abgrenzungsversuche zwischen der Reportage und dem Feature siehe bspw. Weischenberg (2001: 60, 182) oder auch Burger/Luginbühl (2014: 220 ff.). 
auf, die Authentizität, Subjektivität und Individualität kommunizieren sollen. Das in diesem Kontext hierfür aufgewendete Stilmittel ist die Redewiedergabe. In Kompakterzählungen dienen Zitate der Meinungsdarstellung von Personen ${ }^{43}$, die an den Erzählinhalten in irgendeiner Weise teilhaben oder von den erzählten Ereignissen betroffen sind. Im Zitat beziehen die zitierten Personen durch ihre persönliche und bewertende Aussage Stellung zum Erzählten. Das bewirkt eine quantitative Erweiterung des Erzähltextes (z. B. durch Verba dicendi). Somit kann das Optimierungsgebot zugunsten der imagefördernden (S)D eingeschränkt werden. Die Imageförderung besteht letztlich darin, dass die positiven (!) Zitate/Bewertungen auf das Unternehmen bezogen sind. Das folgende Beispiel einer Kompakterzählung von Rewe veranschaulicht den Zusammenhang zwischen dem direkten Redezitat (im Erzähltext fett hervorgehoben) und dessen indirekter Auswirkung auf die (S)D des Unternehmens (Bsp. Zitat als Merkmal eines „Kurz-Features“ in Kompakterzählungen):

„++ Elternzeit - auch ein Thema für Männer ++

14 Monate Elternzeit - das ist eher ungewöhnlich für einen Vater.

[Max] macht es vor! Nach der Geburt seines Sohnes [Jannis] im August 2014 verabschiedete sich der junge Vater für 14 Monate, um sich komplett auf das aufregende erste Jahr des Nachwuchses konzentrieren zu können. Und dieses genießt er nun in vollen Zügen, wie er berichtet:

\section{„Ich vermisse nichts und würde mich jeder Zeit wieder so entscheiden.“}

Um den Anschluss an seinen Traumjob bei der REWE Group nicht zu verlieren und es nach der Elternzeit leichter zu haben, nimmt [Schmidt] weiterhin regelmäBig an Bereichsmeetings teil - ein Tipp, den er allen jungen Eltern, die eine Zeit lang ausschließlich für ihre Kinder da sein möchten, geben kann. (Rewe, Facebook, 2015-03-26. Korpusquelle: REWE_355_FB)

Die Überschrift führt das Thema Elternzeit ein und spezifiziert es als ein geschlechterübergreifendes Angebot - Auch ein Thema für Männer. Nach einer verallgemeinernden Aussage (generalisierende Sentenz), die bereits die Erzählwürdigkeit der Kompakterzählung mit dem Adjektiv ungewöhnlich begründet, folgt die Einführung des Protagonisten Max Schmidt in einer Vorbildfunktion. In

\footnotetext{
${ }^{43}$ Siehe hierzu auch Hausendorf/Kesselheim (2008: 145 f.): Redewiedergabe als Darstellungshinweis.
} 
einem komplexen Satzgefüge wird Max Entscheidung, in die Elternzeit zu gehen, thematisiert, um im Anschluss daran seine Bewertung in doppelter Ausführung wiederzugeben: Nämlich einmal in einem Aussagesatz über Max “ Empfinden (genießen) und einmal in dem wörtlichen Zitat Ich vermisse nichts und würde mich jeder Zeit wieder so entscheiden. Dabei ist der vorangestellte Aussagesatz über Max Emotion eine Interpretation seiner zitierten Rede: Es handelt sich um eine Interpretation von positiven subjektiven Empfindungen des Protagonisten, deren Wahrhaftigkeit das Zitat durch eine absolute Stellungnahme in der Verbalphrase ich vermisse nichts und die temporale Angabe jeder Zeit wieder so entscheiden belegt. So wird neben dem imagefördernden Aspekt - Rewe unterstützt Mitarbeiter/innen, die in Elternzeit gehen möchten - auch der Beleg dafür erbracht, dass diese Möglichkeit in dem Unternehmen aktiv gelebt wird. Das Zitat fördert somit nicht nur eine günstige $(S) D$, sondern stellt im Sinne der Geschichte zugleich einen impliziten Höhepunkt der Erzählung in Form eines persönlichen Erlebnisses dar. Dadurch soll das Minimalkriterium ,the story must contain some element that makes it reportable“" (Labov 1972: 370 f.; van Dijk 1974/1975: 286 f., nach Gülich/Quasthoff 1987: 171) erfüllt werden, da das persönliche Erleben die erzählten Inhalte als ,sozial relevantes außergewöhnliche Ereignis“ (Kindt 1997, nach ebd., vgl. hierzu Abschn. 5.2.1) herausstellt und so als Höhepunkt stilisiert. Die Verschiebung des Höhepunkts in eine positive Bewertung - statt in die Auflösung einer Komplikation - ist eine gängige Praxis in den erhobenen uRE. Damit haben evaluative Elemente mehrere Funktionen: Sie sind nicht nur eine Begründung für die Erzählwürdigkeit, sondern auch implizite Höhepunkte, die auf den Merkmalen ,Subjektivität ${ }^{\text {‘ }}$ und ,Wahrhaftigkeit' gründen, welche wiederum $(S) D$ des entsprechenden Unternehmens stützen (Belegfunktion).

\subsubsection{Schlussgestaltung}

Der Abschluss von Kompakterzählungen nimmt unterschiedliche Formen an und ist selten typisch für Erzählungen. Das untenstehende Balkendiagramm zeigt die verschiedenen Schlussgestaltungen nach ihrem Vorkommen in den untersuchten Kompakterzählungen (Abb. 9.11): 


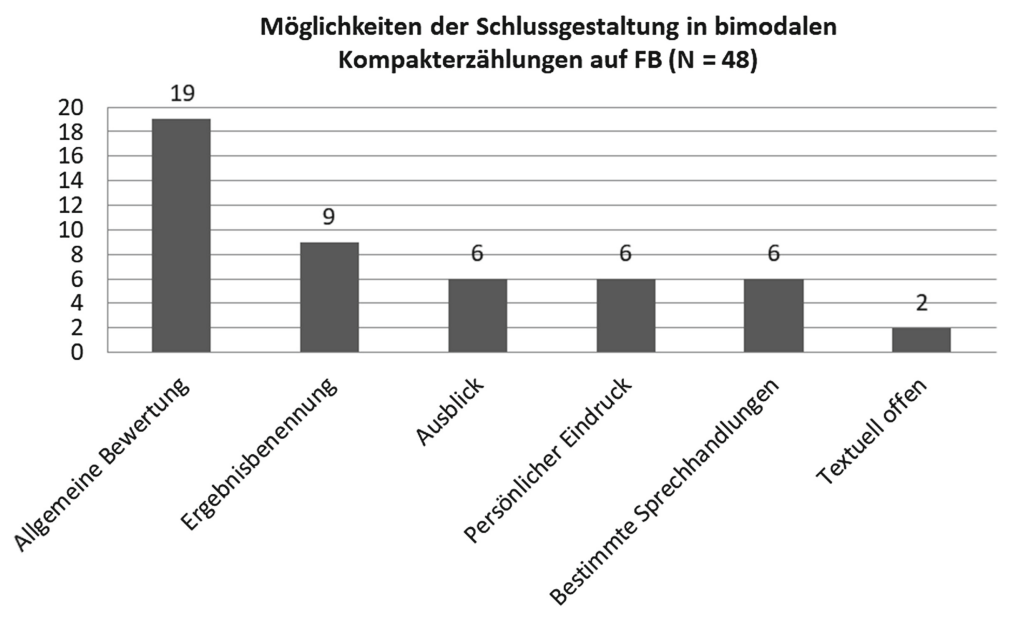

Abb.9.11 Möglichkeiten der Schlussgestaltung bimodaler Kompakterzählungen auf Facebook $(\mathrm{N}=48)$

Am Ende von Kompakterzählungen nutzen die Verfasser/innen die Gelegenheit, die erzählten Inhalte an das gewünschte Merkmal zur (S)D der Unternehmen rückzubinden. Das erreichen sie, indem sie a) einen Mehrwert, den das Unternehmen anderen ermöglicht, benennen und b) ein partnerschaftliches Verhältnis unter den Arbeitnehmer/innen vorführen, das Rückschlüsse auf eine kooperative und wohlwollende Arbeitsatmosphäre nahelegt. Das mündet meist in ein Fazit, eine Schlussfolgerung oder Moral und weniger in ein Resümee im Sinne einer ,zusammenfassende[n] Einschätzung [des Erzählten] vom Erzählzeitpunkt (Gegenwartszeitpunkt) aus“ (Brinker et al. 2014: 66).

Die Mehrzahl der Kompakterzählungen werden mit allgemeinen Bewertungen $(\mathrm{N}=19)$ abgeschlossen. In den erhobenen uRE kennzeichnet sie, dass sie sich immer auf ein Kollektiv beziehen. Sprachlich manifestiert sich das durch den Gebrauch des Personalpronomens wir, das Indefinitpronomen man/alle, Gruppenbenennungen (Team, DH-Studenten) und letztlich die Entpersonalisierung (die Ausbeute, der Spielenachmittag). Die Bewertung basiert dementsprechend auf einer kollektiven Erfahrung, die semantisch in den Wortfeldern ,Glück und Freude' (froh was erreicht wurde, zufrieden sein, große Freude daran, eine Menge Spaß, begeistert) sowie ,Erfolg' (erfolgreich sein, voller Erfolg sein, mit Erfolg, ereignisreich, die Ausbeute kann sich sehen lassen, so passen [...] bestens zusammen) verortet ist. 
Am zweit häufigsten ist die Ergebnisbenennung umgesetzt. Sie basiert auf überprüfbaren „Fakten“. Dadurch entsteht der Eindruck, es läge eine neutrale, lediglich ergebnisorientierte und somit verlässliche Erzählerposition vor. Da sich die Ergebnisse immer auf Erfolge beziehen, ist ihnen immer auch eine abschlieBende Bewertung inhärent. Diese Schlussgestaltung tritt bezeichnenderweise nur in Kompakterzählungen auf, die in der dritten Person verfasst sind. Mit der Ergebnisbenennung vermeiden Verfasser/innen, Rezipient/innen durch eine zu positive (S)D einen Anlass zum Misstrauen zu geben. Solche Abschlüsse sehen beispielsweise wie folgt aus (das Ergebnis ist unterstrichen):

- Eine Mail von Dieter Zetsche sorgt dafür, dass der Wunsch Realität wird - noch dazu mit einer ganz besonderen S-Klasse. (Daimler, Facebook, 2016-08-06. Korpusquelle: DAIMLER_121_FB) $\rightarrow$ Ergebnis: Wunsch wird Realität.

- Mit dem neu gebauten 1.400 Quadratmeter großen Markt in Oelsnitz im Vogtland erfüllte sich dieser Wunsch. Seit März leitet der Quereinsteiger seinen ersten eigenen Markt. (Rewe, Facebook, 2015-06-25. Korpusquelle: REWE_371_FB) $\rightarrow$ Ergebnis: Wunsch wird Realität.

- Nach getaner Arbeit wandten sie ihr erlerntes Wissen an und brachten einer Kindergruppe die Heide nahe. (Rewe, 2015-10-08. Korpusquelle: REWE_353_FB) $\rightarrow$ Ergebnis: Wissensvermittlung an Kinder.

- Und mithilfe der Startgebühr konnten 6.260 Euro gesammelt werden, die das Unternehmen dem Verein [...] gespendet hat. Die Organisation setzt sich für den Kampf gegen seltene Stoffwechselkrankheiten ein. (Rewe, Facebook, 2016-05-23. Korpusquelle: REWE_358_FB) $\rightarrow$ Ergebnis: Geldspende.

- Das Vorstandsmitglied der REWE Group stellte sich allen Fragen [...] gab den Berufsanfängern Tipps aus erster Hand. (Rewe, 2015-10-03. Korpusquelle: REWE_349_FB) $\rightarrow$ Ergebnis: Vorstandmitglied berät Berufsanfänger.

Schlussgestaltungen in Form des Ausblicks zielen darauf, von den Unternehmen herbeigeführte Mehrwerte und positive Arbeitsbedingungen zu kommunizieren. Der Ausblick basiert daher auf den Folgen, die die erzählten Ereignisse hervorrufen. Diese Folgen müssen nicht immer expliziert werden, sie können auch nur angedeutet sein. Die folgenden Beispiele illustrieren dem Abschluss inhärente, spezifische Folgen:

$\rightarrow$ Folge der (erzählten) Ereignisse: freudige Erwartung

- Wir freuen uns schon sehr die neuen Azubis [...] wieder bei der REWE Group zu begrïßen. (Rewe, Facebook, 2016-06-08. Korpusquelle: REWE_360_FB) 
- Vielen Dank für deine Unterstützung und auf ein baldiges Wiedersehen, liebe [...]! (Daimler, Facebook, 2016-02-25. Korpusquelle: DAIMLER_114_FB)

- Nun warten Kinder, Eltern und Betreuer gespannt auf die Ankunft des ersten Storchenpaares. (E.ON, Facebook, 2016-03-11. Korpusquelle: E.ON_661_FB)

- Wir wagen die Prognose von höheren Geburtenraten bei Mensch und Storch! (E.ON, Facebook, 2016-02-23. Korpusquelle: E.ON_658_FB)

$\rightarrow$ Folge der (erzählten) Ereignisse: (berufliche) Weiterentwicklung

- Da ich auch im kaufmännischen Bereich fit werden will, habe ich mich dazu entschieden noch eine zweite Ausbildung anzuhängen. (Rewe, Facebook, 2014-04-30. Korpusquelle: REWE_348_FB)

- Eines steht fest: Ganz nach dem Motto,Let's keep the pace up' ist der Blick bei Daimler in die digitale Zukunftgerichtet. (Daimler, Facebook, 2016-05-28. Korpusquelle; DAIMLER_109_FB)

Kompakterzählungen, die mit einem persönlichen Eindruck abschließen, stehen in Verbindung mit der Realisierungsvariante „Miniatur-Feature“. Bezeichnend dafür ist, dass das „Schlusswort“ sozusagen Betroffenen gehört. Die untenstehende Kompakterzählung verdeutlicht diese Praxis. Sie handelt von einer Mitarbeiterin, die ein Sabbatical bei Rewe in Anspruch genommen hat (Schlussgestaltung ist fett hervorgehoben).

++ REWE Group-Sabbatical: Auszeit als Festangestellte in Down Under ++

REWE Group-Mitarbeiter können seit 2012 Sabbaticals in Anspruch nehmen. Eine, die es bereits genutzt hat, ist Susi. Als die Logistik-Sachbearbeiterin ins Flugzeug nach Australien stieg, wollte sie den Sommer verlängern. Nicht für eine oder zwei Wochen, sondern für mehrere Monate. Das Besondere: Als festanagestellte Mitarbeiterin erhielt sie Dank eines Gehaltssplittings während der Freistellung ein angepasstes Gehalt und war somit weiterhin sozialversichert. „Die Zeit war unbeschreiblich und die Erinnerung an Erlebtes sind unbezahlbar. Und die Sicherheit, in seinen Job zurückzukönnen, ist ein unheimlich gutes Gefühl.“ erzählt die 29-Jährige.

Weitere Informationen zu REWE Group als Arbeitgeber finden Sie hier: [Link] (Rewe, Facebook, 2015-11-19. Korpusquelle: REWE_372_FB)

Die eigentliche für die (S)D relevante Information - Rewe stellt seinen Mitarbeiter/innen durch Sabbaticals eine berufliche Auszeit zur Verfügung - ist 
in die personifizierte Erzählung über die Rewe-Mitarbeiterin Susi eingearbeitet. Am Ende steht ein direktes Zitat von ihr, in dem sie das Sabbatical retrospektiv bewertet. Es ist gekennzeichnet von dem affektiven Ausdruck ihres außergewöhnlich positiven Erlebnisses (unbeschreiblich, unbezahlbar), verbunden mit der ebenfalls positiven Erfahrung mit dem Arbeitgeber (unheimlich gutes Gefühl), der ihr das Erlebnis durch die Sicherheit in den Job zurückzukönnen erst ermöglicht hat. Die Vermittlung von Erfahrungshaftigkeit stilisiert auch hier die Information als erzählenswert. Das Zitat impliziert die Authentizität der erzählten Inhalte und stützt so die imagefördernde (S)D des Unternehmens: Wir sorgen für unsere Mitarbeiter/innen. Auf das resümierende Zitat folgt ein Verlinkungsangebot zu weiterführenden, detaillierten Informationen. Daimler fügt dem Abschluss von Kompakterzählungen, die inhaltlich im Rahmen von Ausbildungs-/Berufsfeldbeschreibung und/oder -erfahrung entstehen, die direktive Sprechhandlung ,Fragen" hinzu, der der Link sozusagen als Antwort im imaginierten Dialog folgt, z. B.: Neugierig geworden? Ihr habt auch Interesse an einer Industrie-Promotion? Ihr möchtet auch DoktorandIn bei Daimler werden? Bezeichnend dafür ist die informelle ,Du- ' bzw. ,Ihr- ' Anrede, während Kompakterzählungen, die sich nicht an potenzielle Mitarbeiter/innen wenden, den Link ausschließlich über das Informationsangebot ankündigen:

- Mehr im Daimler-Blog [Link-Adresse] (Daimler, Facebook, 2016-08-06. Korpusquelle: DAIMLER_121_FB)

- E.ON ,Mehr Info hier: [Link-Adresse] (E.ON, Facebook, 2016-02-23. Korpusquelle: E.ON_658_FB)

- EnBW „Mehr dazu auch in der Schwäbischen Zeitung: [Link-Adresse] (EnBW, Facebook, 2014-08-21. Korpusquelle: 709_FB)

Unter die Schlussgestaltung durch ,bestimmte Sprechhandlungen“ fallen der Ratschlag, der Wunsch und die Begründung. Mit diesen Sprechhandlungen können sowohl am Sachverhalt Interessierte als auch Akteure der Erzählung adressiert werden. Der Ratschlag ist auf der lexikalischen Ebene anhand des umgangssprachlichen Substantivs ,Tipp` ausgewiesen:

- - ein Tipp, den er allen jungen Eltern [...] geben kann. (Rewe, Facebook, 2015-05-26. Korpusquelle: REWE_355_FB)

- Unser Tipp: Meide die Küste, Olli! (E.ON, Facebook, 2015-08-29. Korpusquelle: E.ON_652_FB) 
Den ,Wunsch“ indizieren die Verben, wünschen “ und ,hoffen`:

- Liebe [...], die Azubi-Redaktion wünscht Dir dafür weiterhin alles Gute! (Rewe, Facebook, 2016-06-24. Korpusquelle: REWE_368_FB)

- Wir hoffen auf baldigen Falkennachwuchs! (E.ON, Facebook, 2015-05-17. Korpusquelle: E.ON_663_FB)

- Die EnBW wünscht viel Spaß beim Kicken bis in die späten Abendstunden! (EnBW, Facebook, 2016-07-22. Korpusquelle: EnBW_719.1_FB)

Die ,Begründung ' leitet in den Daten letztlich eine Präpositionalphrase ein:

- Um sich wieder heimisch zu fühlen, hat die E.ON Tochter E-DIS AG in Absprache mit dem Naturschutzbund $\mathbf{5 0 0}$ Nisthilfen errichtet. (E.ON, Facebook, 2015-04-14. Korpusquelle: E.ON_66_FB)

Lediglich zwei der Kompakterzählungen weisen keine der genannten sprachlichen Abschlussgestaltungen auf. In dem obigen Balkendiagramm sind sie als ,textuell offen “ dokumentiert. Die Spezifizierung ,textuell“ deutet bereits an, dass der Abschluss einer Kompakterzählung nicht immer sprachlicher Art sein muss. Wie bereits erwähnt, sind - mit Ausnahme von Kompakterzählungen auf der Plattform Xing - allen erhobenen Kompakterzählungen Fotografien beigefügt. Das mag zum einen an Rezeptionserwartungen bezogen auf die Internetplattformen liegen und strategischen Erwägungen wie der Aufmerksamkeitslenkung dienen. Zum anderen sind Bilder aber auch eine weitere semiotische Ressource, die im narrativen Gefüge funktional sein kann.

\subsubsection{Text-Bild-Gefüge in bimodalen Kompakterzählungen}

Die neuen technologischen Möglichkeiten im Internet stellen auch die Textlinguistik vor neue Herausforderungen. Ausgehend von der Entwicklung herrscht in der Linguistik Konsens, dass die zunehmende Präsens außersprachlicher Zeichen in Texten erfordert, sich auf alle semiotischen Systeme zu beziehen, „die neben dem Zeichensystem Sprache in der Struktur eines Textes manifest werden“ 
(Heinemann/Viehweger 1991: 125) ${ }^{44}$ (vgl. auch Abschn. 3.1). Ein semiotisches System, das für bimodale Kompakterzählungen von grundlegender Bedeutung ist, sind Fotografien. Daher stellen sie einen wichtigen Bestandteil der Untersuchung dar. Es ist ,unabdingbar, die (audio-)visuellen Elemente jedweder Art, sofern sie für die Interpretation von Kommunikationsangeboten relevant sind, in die (Text-) Analyse einzubeziehen“ (Brinker et al. 2014: 22) ${ }^{45}$. Von Interesse sind in dem hiesigen Kontext vorrangig drei Fragen, die aufeinander aufbauen:

1. Auf welchen gemeinsamen formalen Merkmalen basieren die Fotografien in den untersuchten Kompakterzählungen?

2. Wie wird intermodale Kohärenz zwischen dem Erzähltext und den Fotografien hergestellt?

3. Inwiefern sind die Fotografien ein konstitutives Element in Erzähltexten bimodaler Kompakterzählungen?

Für die Untersuchung ist der Blick darauf leitend, inwieweit sich Synergien aus der Verbindung von Text und Fotografien für bimodale Kompakterzählungen ableiten lassen; also wie die beiden semiotischen Systeme sich in den Erzählungen arbeitsanteilig ergänzen. Das ist daher von Interesse, da die Kompakterzählung sich durch Kürze - basierend auf textueller Komprimierung auszeichnet. Somit ist zu erwarten, dass Bilder mögliche Lücken füllen und/oder die Erzähltextinhalte spezifizieren. Das berührt sowohl informationsbezogene und global-semantische Aspekte (welchen Beitrag die Verbindung von Text und Bild im Rahmen einer Gesamtbotschaft leisten) als auch Aspekte der visuell/verbalen Rhetorik und pragmatischer Funktionen (Formen der gegenseitigen Bezugnahme).

\footnotetext{
${ }^{44}$ In dem Sammelband Brauchen wir einen neuen Textbegriff? Antworten auf eine Preisfrage (Fix/Adamzik/Antos/Klemm 2002) sprechen sich die meisten Beitragenden für eine weite Definition von Text aus. Daraus folgen Textauffassungen mit verschiedenen Gewichtungen der Interaktion von sprachlichen und außersprachlichen Zeichen (vgl. Opilowsik 2013: 119). ${ }^{45}$ Somit schließe ich mich einer weiten, semiotisch verstandenen Sichtweise des Textbegriffs an, wie er in den Sprach-, Kommunikations- und Medienwissenschaften mittlerweile üblich ist (Stöckl 2004a: 244).
} 


\subsubsection{Merkmale der beigefügten Fotografien und intermodale Kohärenzbildung}

Jedem Facebook-Post können bewegte und unbewegte Bilder in Alben angehängt werden. Diese Bilder und Alben folgen dann, anders als beispielsweise in Corporate Blogerzählungen, dem Fließtext. Das bedeutet, die Bilder treten am Ende der Erzähltexte von Kompakterzählungen auf. Der mögliche Umfang für das Hochladen von Bildern auf Facebook ist mit 1.000 Fotos pro Album sehr umfangreich (Facebook (2018), Fotos und Dateien). Die Option, ein solch umfangreiches Album anzulegen, ist insbesondere für private Facebook-Nutzer/innen interessant. Im Zusammenhang mit Kompakterzählungen würde ein großes Album jedoch über das Ziel hinausschießen: Denn wer wäre motiviert und würde sich die Zeit nehmen, in diesem Kontext ein sehr umfangreiches Fotoalbum in Gänze anzusehen? Grundsätzlich schwankt die Anzahl der auf Facebook hochgeladenen Fotografien pro archivierter Kompakterzählung zwischen einem und neun Bildern $^{46}$. Dabei sind unternehmensspezifische Präferenzen zu beobachten. In den vorliegenden Kompakterzählungen zeigt sich, dass Rewe (mit $\mathrm{N}=16$ ) und die EnBW (mit N = 8) überwiegend ein Foto anfügen. Daimler postet für gewöhnlich mehr als ein Foto, geht dabei allerdings nicht über die Anzahl von vier Fotografien hinaus. Der Vorteil von vier Bildern pro Kompakterzählung ist, dass alle Fotografien mit dem dazugehörigen Post auf einen Blick zu sehen sind. Alles was über vier Bilder innerhalb eines Posts hinausgeht, fordert von den Rezipient/innen einen Mehraufwand, falls sie die folgenden Bilder auch betrachten möchten. Sind wie beispielsweise bei Rewe (siehe die Abb. unten) neun Fotografien vorhanden, müssen die Rezipient/innen sie direkt anklicken. Dabei zeigt das letzte Foto in der unteren rechten Ecke mit dem ,,+“-Symbol an, wie viele Bilder zusätzlich hochgeladen wurden (Abb. 9.12):

\footnotetext{
${ }^{46}$ Bewegte Bilder (also Videodateien) sind in den Daten unüblich. Diese treten eher bei Twitter (in Diskontinuierlichen Erzählungen), auf Homepages („Gründer“- und „Unternehmensgeschichte) von Unternehmen und Corporate Blogs auf. Die Bildfunktion von Corporate Blogerzählungen ist mit der in Kompakterzählungen nahezu identisch.
} 
exw. REWE Group Karriere

Cumner Oitober 2015 .

++ Azubis auf der Dellbrücker Heide ++

Was ist eine Heide und welche Pflanzen und Tiere leben dort? Dieser Frage sind unsere Anja, Claudia, Mari, Hilde, Heiko u. Peter nachgegangen. Henrike und Sonja vom Umweltverband BUND führten sie durch die Heide. Unerwünschte Gewächse wie die Amerikanische Traubenkirsche vertreiben das Heidekraut und andere gefährdete Pflanzenarten. Unsere Azubis griffen zur Heckenschere und schnitten die unliebsame Traubenkirsche zurück, um den Lebensraum für Heidekraut \& Co. wiederherzustellen. Nach getaner Arbeit wandten sie ihr erlerntes Wissen an und brachten einer Kindergruppe die Heide nahe.

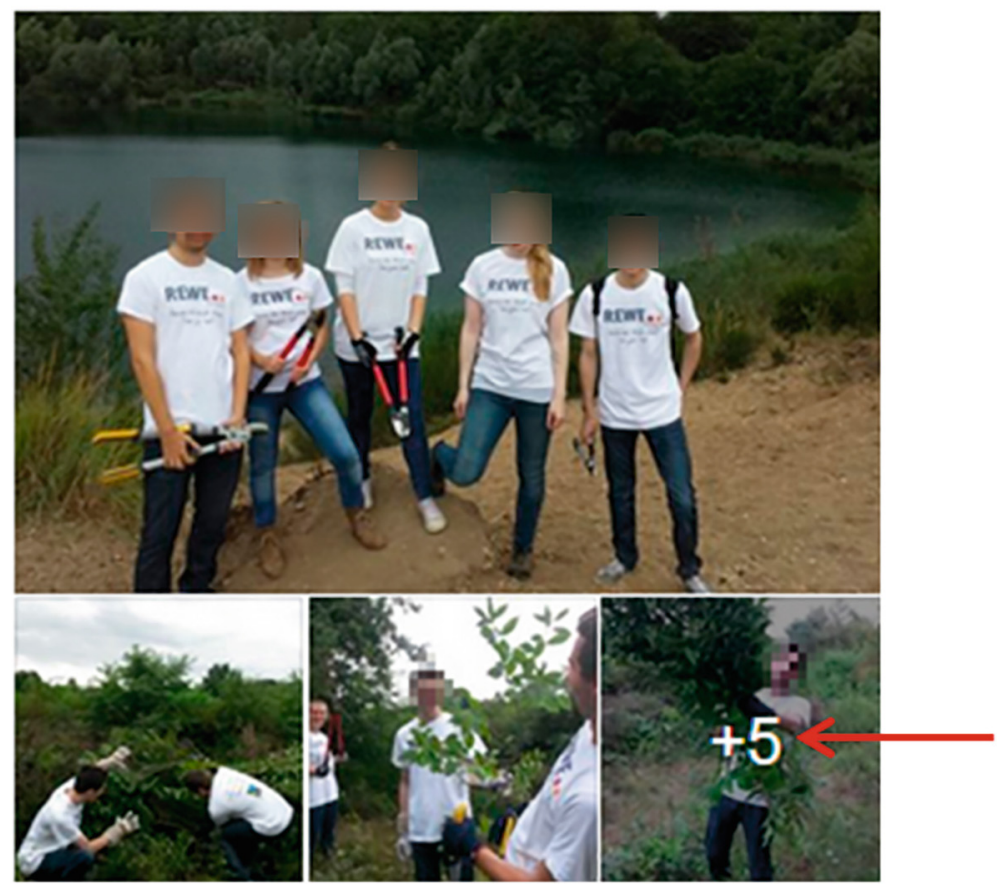

Abb.9.12 Facebook-Kompakterzählung mit neun beigefügten Fotografien (Rewe, Facebook, 2015-10-08. Korpusquelle: REWE_353_FB) 
Nach Sichtung der realisierten Motive der angefügten Fotografien wird deutlich, dass die Fotografien überwiegend Personen zeigen (Personen, $\mathrm{N}=75$; Objekte/Tiere, $N=25$ ). Das legt nahe, dass es üblich ist, in erster Linie die Protagonist/innen einer Kompakterzählung abzubilden: entweder einzeln, in Gruppen als Portrait (statisch) oder bei bestimmten Tätigkeiten (dynamisch). Betrachtet man, welche Einstellungen für die Aufnahmen gewählt werden, handelt es sich in der Mehrzahl um Nah-Aufnahmen (medium close up, Bordwell/Thompson 2013: 190) $(\mathrm{N}=17 \text {, siehe unten) (Abb. } 9.13)^{47}$

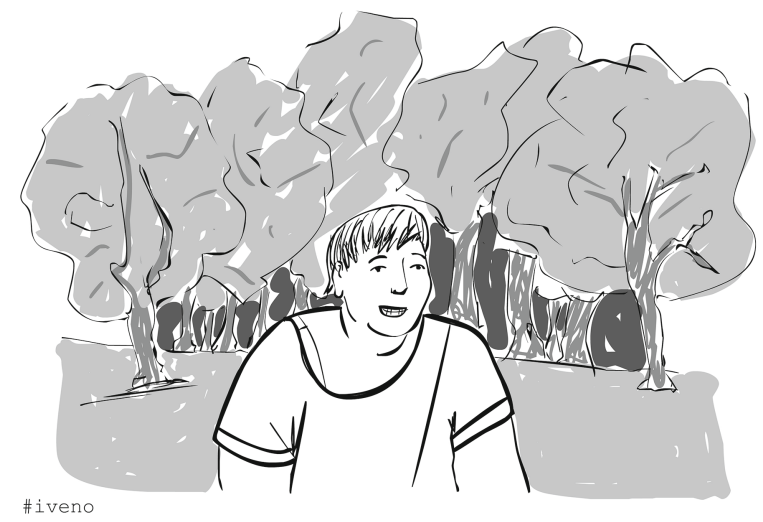

Abb.9.13 Nah-Aufnahme (medium close up, Bordwell/Thompson 2013: 190) (Rewe, Facebook, 2014-09-03. Korpusquelle: REWE_373_FB)

und um Halbtotale-Aufnahmen (long shot, Bordwell/Thompson 2013: 190) (N $=22$, siehe folgende Abb.) (Abb. 9.14).

Betrachtet man die Erzähltexte und Bilder aus einer informationsbezogenen und global-semantischen Perspektive, zeigt sich, dass der Inhalt der Kompakterzählung mit der gewählten fotografischen Einstellung in Verbindung steht. Nah-Aufnahmen, die vom Kopf bis zu der Mitte des Oberkörpers reichen, werden beispielsweise dann gewählt, wenn etwas von oder über eine bestimmte Person erzählt wird. Die Großaufnahme rückt die Einzelperson, insbesondere den Gesichtsausdruck in den Mittelpunkt. Indirekte und direkte Aussagen, die mit der abgebildeten Person in Verbindung gebracht werden, ,tragen dabei zur

${ }^{47}$ Die Fotografie wurde zur Wahrung der Persönlichkeitsrechte verfremdet. 


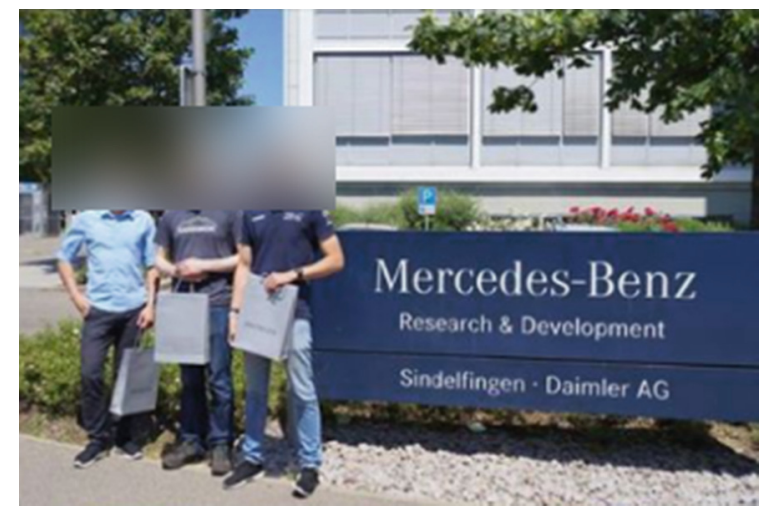

Abb.9.14 Halbtotale-Aufnahme (long shot, Bordwell/Thompson 2013: 190) (Daimler, Facebook, 2016-07-2. Korpusquelle: Daimler_113_FB)

Perspektivierung und Beurteilung ihrer Aussage durch den Leser bei“ (Stöckl 2004a: 272). Bei dem oben angeführten Beispiel einer Nah-Aufnahme von Rewe (die Fotografie musste verfremdet und nachgezeichnet werden) geht es im Erzähltext darum, dass die abgebildete Person ein Sabbatical in Anspruch genommen hat. Die junge Frau trägt ein lässiges T-Shirt und ist in einer bequemen Haltung (leicht nach vorne gebeugt) abgebildet. Sie lächelt fröhlich in die Kamera. Ihre Haare sind „,verweht“, die Haut ist sonnengebräunt. Aufgrund der Schatten im Hintergrund sehen wir, dass die Sonne scheint. Der sandige Untergrund lässt annehmen, dass sich die fotografierte Person an einem Strand (vermutlich während ihres Sabbaticals) befindet. Diese „Bühne“ suggeriert in Verbindung mit dem zufriedenen Gesichtsausdruck Entspannung und deutet auf das wohlig angenehme Lebensgefühl zum Zeitpunkt der Aufnahme hin. Das Foto lädt den Erzähltext mit Empfindungen wie Urlaubsgefühlen, Freiheit, Muse und Entspannung auf, während der Text dazu passende Aktivitäten benennt (Segeltörns, Kennenlernen neuer Mitmenschen), ohne diese jedoch narrativ auszuführen. Das positiv konnotierte Foto lässt Raum für Assoziationen. Das Ende der Kompakterzählung bilden zwei übergreifende textuelle Bewertungen der Protagonistin (Zeit-das war wohl das größte Geschenk... und eine solche Erfahrung [ist] eine echte Empfehlung). Dahinter steht die imagefördernde (S)D des Unternehmens, nicht nur Rücksicht 
auf die Work-Live-Balance seiner Angestellten zu nehmen, sondern sie auch aktiv zu fördern. ${ }^{48}$

Die intermodale Kohärenz konstituiert sich, da wir bestrebt sind, Bedeutung aus dem dargebotenen Text und Bild nach folgendem Prinzip zu generieren:

„Was gleichzeitig oder benachbart sprachlich und bildlich wahrnehmbar gemacht wird, wird wohl schon etwas miteinander zu tun haben, so darf nach der allgemeinen Griceschen Kooperationsmaxime vermutet werden." (Holly 2009: 364). ${ }^{49}$

Dabei stellen wir semantische Bezüge zwischen den sprachlichen und den bildlichen Informationen her. Basierend auf der räumlichen Nähe, verbinden wir dann sprachliche Merkmale mit bildlichen. Die Übereinstimmung der Zahl der Protagonist/innen in den Erzähltexten mit der Zahl der abgebildeten Personen erlaubt die Person/en direkt mit dem Erzähltext zu verbinden und anhand der Nah-Aufnahme Schlüsse über das Befinden der Protagonist/innen in der Situation abzuleiten (kognitiver Vorgang, basierend auf unserem Weltwissen). Dadurch entsteht eine implizite Bewertung, die sich durch die Verknüpfung von Aussagen im Erzähltext mit dem dazugehörigen Foto manifestiert: Aus den Bildern werden so textgeleitet evaluative Momente herausgelesen. Die Fotografien haben Anteil am Zustandekommen der (S)D, da sich Text und Bild nicht nur ergänzen und „wechselseitig erhellen“ (d. h., ,Text und Bild erläutern einander“, Schmitz (1999: 16)), sondern die Gesamtbotschaft der Kompakterzählung auf Basis eines bildlichen Eindrucks holistisch interpretiert werden kann (vgl. Nöth 2000: 493): Der Beitrag solcher Bilder zu der Erzählung ist also, dass sie zu sprachlich geäußerten Handlungen und Fakten Personenmerkmale und situative Assoziation bereitstellen, die über das im Erzähltext verschriftete Zeichen hinausgehen. Die Erzähltexte gewinnen dabei an Glaubwürdigkeit, da die Text-Bild-Bezüge bildsemantisch in eine Sprachauthentisierung (Holly 2009: 370) münden ${ }^{50}$. Daraus

\footnotetext{
48 Andere „Nah“-Aufnahmen von Personen werden bspw. zu Erzählungen über den beruflichen Werdegang von Mitarbeiter/innen gepostet. Auch hier sind gut gekleidete, fröhlich und entspannt wirkende Personen zu sehen, die in der (S)D des Unternehmens unterstützt und gefördert wurden.

${ }^{49}$ Schmitz (1999) argumentiert ähnlich, wenn er die Zusammengehörigkeit von Text und Bild auf die „Einheit des Ortes“ (1999: 5) zurückführt, wodurch die „versammelten Zeichen einen sinnvollen Zusammenhang ergeben“ (ebd.).

${ }^{50}$ Stöckl (2004a: 272) fasst die Figurenrede des Sprache-Bild-Bezugs, der den/die Urheber/in des Textes abbildet (im Fall der Kompakterzählung der/die Urheber/in des direkten oder indirekten Zitats), unter dem Comicmuster. Der Vorteil daran ist nicht nur die Visualisierung der Figur und ihrer Merkmale, sondern auch, dass der kognitive Aufwand während der Integration von Bild- und Textinhalt keinerlei spezifische kognitive Anforderungen an
} 
resultiert allerdings für die Kompakterzählung, dass die sprachliche Evaluation mit den bildlichen Zeichen (Abbildung des Zitierten) im Einklang sein muss. Ist das gegeben, plausibilisieren die Fotografien nicht nur das Geschriebene, sondern regen die Leser/innen dazu an, Eindrücke und Aussagen aus der Kompakterzählung nachzuempfinden. Damit tragen Fotografien wesentlich zur Vermittlung von Erfahrungshaftigkeit (Fludernik 1996, Abschn. 5.2.2) bei, die bezogen auf die imagefördernde (S)D in Kompakterzählungen eine persuasive Funktion innehat (siehe hierzu insbesondere den Grundtyp 4 der (S)D ,attraktiver Arbeitgeber', Abschn. 8.6).

Handelt es sich um Fotografien, die Personen im Dialog - beispielsweise auf einer Messe - zeigen (wie im nächsten Beispiel), wird eine Einstellung zwischen der Nah-Aufnahme und der Halbtotalen-Aufnahme gewählt. Die Einstellung tendiert zu der sogenannten Amerikanischen-Einstellung (medium shot, Bordwell/Thompson 2013: 190). Die Kameraperspektive geht bei solchen Gruppierungen im Dialog üblicherweise über die Schulter eines Gruppenmitglieds (Over-the-shoulder-shot, Goldmann: https://redgo.tv/die-five-shot-regel/). Sie ahmt so die Sicht der Betrachtenden auf die Gruppe nach (siehe unten). Das versetzt die Bildbetrachter/innen in eine Beobachterperspektive und ist somit eine Involvierungsstrategie hinsichtlich der Rezipient/innen, die über das geschriebene Wort hinausgeht (Abb. 9.15).

\author{
Abb. 9.15 \\ Over-the-shoulder-shot \\ einer Gruppe am Daimler \\ Doktorandentag im \\ Gespräch (Daimler, \\ Facebook, 2015-11-20. \\ Korpusquelle: \\ DAIMLER_111_FB)
}

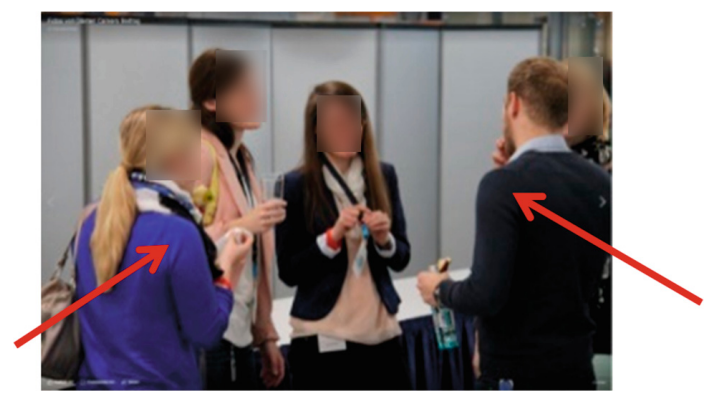

Halbtotale-Aufnahmen, die Menschen von Kopf bis Fuß zeigen (siehe unten), beziehen sich auf kleinere Gruppen, die meist für das Foto in Aufstellung gebracht wurden. Auch hier liegt es nahe, dass es sich um Protagonist/innen

die Rezipierenden stellt, ,,da wir anthropologisch darauf trainiert sind, Sprecher (Physiognomie, Mimik, Gestik, Körperhaltung) und Äußerung als zusammengehörig wahrzunehmen“ (Stöckl 2004a: 273). 
aus der Erzählung handelt. In dem vorangegangenen Beispiel einer HalbtotalenAufnahme im Daimlerpost sind das drei Studierende, die an einer Führung durch das Mercedes-Werk in Sindelfingen teilnehmen durften. Bei dem folgenden Beispiel von Rewe (siehe unten) sind es fünf Auszubildende (Azubis), die unter Anleitung von zwei Angestellten des Umweltverbandes BUND die Dellbrücker Heide von unerwünschten Gewächsen befreien. Durch die Halbtotale-Aufnahmeeinstellung liegt der Fokus - im Gegensatz zu der NahAufnahme - stärker auf der Rewe-Azubi-Gemeinschaft. Es geht weniger um das spezifische Erleben einzelner Personen, sondern vielmehr um das Präsentieren einer Wir-Gemeinschaft, die üblicherweise Erkennungsmerkmale des Unternehmens aufweist (Markenemblem, Slogans, Projektnamen oder Unternehmensbezeichnung). Die zwei Angestellten des Umweltverbandes sind nicht Bestandteil des ersten Gruppenbildes. Sie erscheinen erst auf dem siebten Bild des Fotoalbums (Abb. 9.16).

Abb.9.16 HalbtotaleGruppenaufnahmen, Rewe-Azubi-Aktion „Pflege der Dellbrücker Heide“" (Rewe, Facebook, 2015-10-08. Korpusquelle: REWE_353_FB)

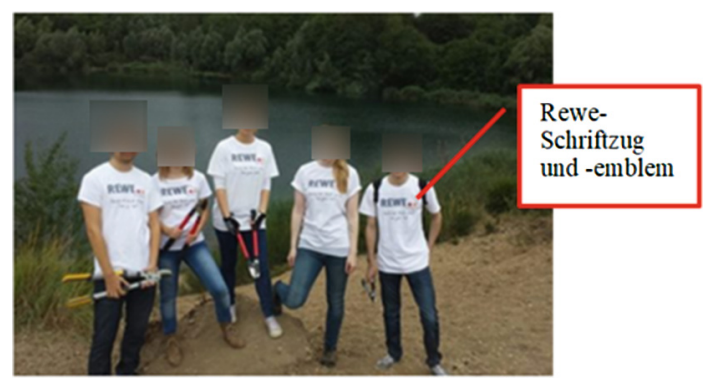

Unternehmensmerkmale auf den Fotografien betonen, dass diese Gemeinschaft im Unternehmenskontext besteht. Die Personengruppe steht dann als Pars pro Toto (Teil für das Ganze). Positive Schlüsse und Merkmale, die dem Foto entnommen werden können (z. B. offene, freundliche, motiviert wirkende Personen), sollen ebenso anderen Unternehmensmitgliedern und letztlich dem Unternehmen selbst zugeschrieben werden. 
Das unternehmensübergreifende Vorkommen der beschriebenen Kameraeinstellungen verdeutlicht, dass die auf den ersten Blick als Schnappschüsse und Amateuraufnahmen anmutenden Fotografien nach bestimmten Gestaltungsvorgaben entstehen. Die Vorgaben setzten jedoch eine bestimmte Expertise der Fotograf/innen voraus, die sie durch die „Machart“ verschleiern. Dabei setzen sie auf den Glaubwürdigkeitsbonus (Holly 2009: 372) von Bildern, der Betrachter/innen meist wider besseren Wissens überzeugt. Das Ergebnis verdeutlicht, wie zielgerichtet und strategisch die Konzeption dieser uRE ausgerichtet ist. Das lässt annehmen, dass jedes Foto gezielt für die Kompakterzählung erstellt wird. Abschließend unterstreicht das Untersuchungsergebnis die Bedeutung und den Beitrag von Fotografien für die Kompakterzählung an sich und rückt die Funktion der Kompakterzählung für die imagefördernde (S)D von Unternehmen in den Vordergrund.

\subsubsection{Narrative Merkmale der Text-Bild-Konstellation}

Grundsätzlich ist es üblich, auf dem ersten Foto einer Kompakterzählung Personen, die in den Erzähltextinhalten auftreten, vorzustellen. Bezogen auf das narrative Gefüge spezifizieren und ergänzen Fotografien also die Situierung, indem sie die Akteure und Protagonist/innen abbilden und dadurch zusätzliche Assoziationen begünstigen (positive Konnotationen, wie oben beschrieben). Danach erst folgen szenische oder dynamische Fotografien, in denen Protagonist/innen in situ Tätigkeiten nachgehen, und/oder von Objekte (z. B. Gebäude, Nahrungsmittel, Handwerkszeuge u. a.) in Groß- (close up, Bordwell/Thompson 2013: 190) oder Detail-Aufnahmen (big close up, Bordwell/Thompson 2013: 190) gezeigt werden.

Die folgenden Bilder aus der bimodalen Kompakterzählung von Daimler über drei Studierende dienen als Beispiel dafür, wie Fotografien nach dem Gestaltungsprinzip der „visuellen Repetition“ (Gaede 1992: 40) textuelle Zeichen der Kompakterzählung in Form, Struktur und abgebildeter Handlung visuell wiederholen. Das Wiederaufnahmeprinzip der narrativen Themenentwicklung kann somit auch zwischen Text und Bild erfolgen. Die Bildabfolge ist dabei an der linearen Erzähltextabfolge orientiert. Inwiefern die Textabfolge die Anordnung der Bilder betrifft, erläutere ich anhand der folgenden Abbildung. Die Bilder nehmen den Erzähltext am Ende sozusagen noch einmal auf. Um das nachvollziehen zu können, befinden sich neben den Bildern graue Kästen. Sie sind an der Abfolge der narrativen Phasen (Situierung, Ereignisrepräsentation, Abschluss) im Erzähltext ausgerichtet und entsprechend durchnummeriert. Auf welche narrative Phase das jeweilige Bild referiert, ist in schwarzer Schrift festgehalten. Das dazugehörige Zitat aus dem Erzähltext steht in grauer Schrift in eckigen Klammern. Die 
Pfeile zeigen, welcher sprachliche Ausdruck visuell wiederaufgenommen wird (Abb. 9.17):

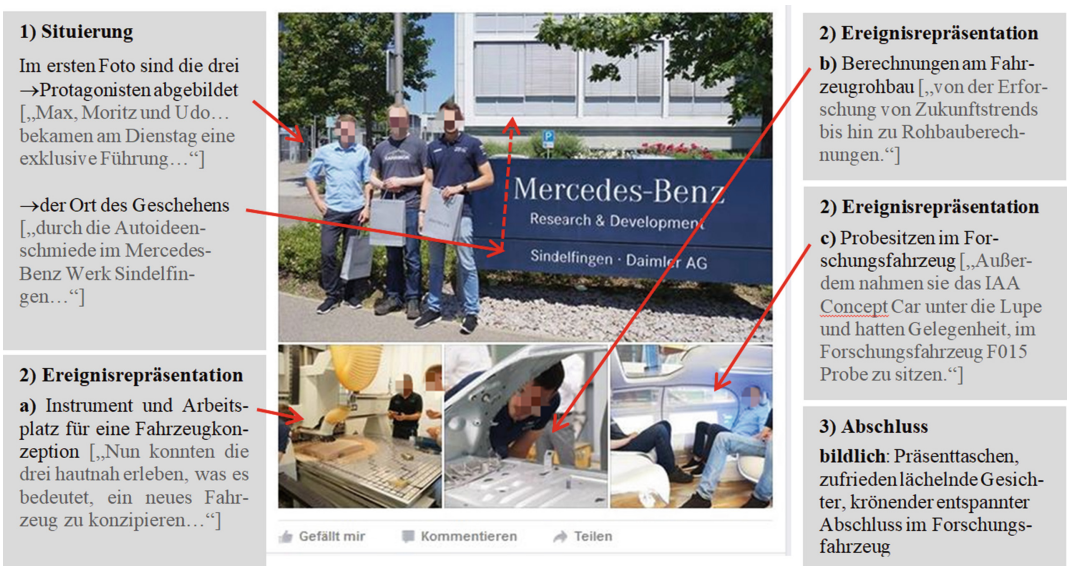

Abb.9.17 Sequenzielle Abfolge und Wiederaufnahme von Elementen aus der Kompakterzählung (Daimler, Facebook, 2016-07-21. Korpusquelle: Daimler_113_FB)

Bezeichnend für die hier angeführten Fotografien ist, dass die Bilder ohne Text wenig Sinn ergeben. Erst der Erzähltext spezifiziert, um was es sich bei den Fotografien handelt. Stöckel (2004a) nennt diese Sprache-Bild-Beziehung Parallelisierung. Er versteht darunter, ,dass das Bild einen im sprachlichen Text benannten Gegenstand oder Sachverhalt (bzw. mehrere) zeigt“ (2004a: 254). Die Parallelisierung stellt seiner Aussage nach den Normalfall dar. Kennzeichnend dafür ist, dass der Text relativ autark ist, da wir ihn auch ohne das Bild nachvollziehen können. Stöckl schließt in diesen Fällen jedoch nicht aus, dass die Bilder dem Text durchaus auch andere Bedeutungsnuancen hinzufügen können. Im Fall von Kompakterzählungen veranschaulichen sie den Erzähltext (Sprachveranschaulichung, Holly 2009: 370), insofern als die Bilder zeigen, was der Erzähltext aufgrund der Komprimierung meist nur aufzählt, aber nicht weiter ausgeführt (seien es Aktivitäten, Gegenstände oder affektive Komponenten).

Da die Bilder in bimodalen Kompakterzählungen nicht linear mit dem Erzähltext verlaufen, nehmen sie Aspekte der Situierung sozusagen am Ende des Erzähltextes erneut auf. Ebenso verhält es sich mit Fotografien, die Aktivitäten aus der Kompakterzählung bildlich vorführen. Die Bilder legitimieren sich 
erst durch den Erzähltext und reichern die Ereignispräsentation mit weiteren Details an, die ebenfalls erst nach dem Text angeboten werden. So entsteht eine inhaltliche Verschränkung zwischen den Fotografien und den dazugehörigen strukturellen narrativen Grundkategorien, denn die Bilder erschließen sich nur mit Bezugspunkten aus dem dazugehörigen Erzähltext. In dieser Konstellation bietet der Erzähltext den Rahmen und lenkt die Bildinterpretation. Die Fotografien führen dabei zu einer ,intensivierte[n] Semantisierung der Wahrnehmungsgegenstände“ (Spangenberg 1988: 784, nach Holly 2004: 128). Das Zusammenspiel von Text und Bild stützt wiederum Facetten der (S)D in Form der Beleg- Beweisführung durch Augenschein (implizite Wirklichkeitsnähe von Fotografien), was auch die Glaubwürdigkeit der kommunizierten (S)D stützt (Wahrheitsgehalt einer realen Begebenheit). Somit sind textuelle und visuelle Elemente der Kompakterzählung bei der Konstruktion der Gesamtbotschaft und somit auch der dadurch kommunizierten imagefördernden (S)D eng verbunden. Die intermodale Kohärenzstiftung erfolgt durch räumliche Nähe und sprachliche Indikatoren (Personennamen, Personengruppen, Objektbenennungen) wie auch durch die semantische Passung zwischen Text und Bild. Explizite bilddeiktische Ausdrücke treten in den vorliegenden Kompakterzählungen nicht auf. Entsprechend konstruieren Rezipient/innen, basierend auf ihrem Kohärenzbedürfnis, einen Zusammenhang zwischen beiden Darstellungsvarianten und ,integrieren sie im Rahmen eines Gesamttextes“ (Stöckl 2004a: 264) - vorausgesetzt die semantischen Merkmale weisen Gemeinsamkeiten auf.

Abschließend lässt sich festhalten, dass Bilder in Kompakterzählungen für sich allein nicht narrativ sind. Um als narrativ gewertet werden zu können, müssten sie zumindest eine erkennbare Veränderung einer Situation oder eines Zustands darstellen (Schmid 2005: 13). Diese geht aus den beigefügten Bildern selbst nicht eindeutig hervor, da sich erst in Kombination mit dem Text die Situations- und/oder Zustandsveränderung erkennen lässt. Allerdings stellen Fotografien etwas ikonisch dar, was andernfalls mit einem deutlich größeren Aufwand versprachlicht werden müsste (z. B. Emotionen, Darstellung von Akteuren, Wirkung bestimmter Ergebnisse auf die Protagonisten, Schauplätze u. a.). Die Fotografien fügen den Kompakterzählungen insbesondere in Hinblick auf die (S)D Bedeutungen zu, die im Erzähltext so nicht expliziert werden. 


\subsubsection{Einordnung in den erzähltheoretischen Kontext}

Neben den zuvor benannten, realisierungsbedingten Merkmalen, die ausschlaggebend für die Namensgebung sind, lassen sich (bimodale) Kompakterzählungen mittels bestimmter narratologischer Eigenschaften spezifizieren. Ich zähle (bimodale) Kompakterzählungen als eine von zwei Realisierungsvarianten zu den Small Stories (Bamberg/Georgakopoulou 2008), was ich in erster Linie auf ihren materialen Umfang zurückführe. Möchte man Small Stories in den sozialen Medien aus der erzähltheoretischen Perspektive erfassen, bietet sich der Ansatz von Page (2015: 333) an, die zu diesem Zweck die fünf narrationsspezifischen Merkmalsdimensionen von Ochs und Capps (2001: 22 ff.) aufgreift (vgl. Abschn. 4.2).

Die Merkmalsdimensionen nach Ochs/Capps ermöglichen es, das narrative Vermittlungsvorkommen in den sozialen Medien im Rahmen narrationsrelevanter Spektren zu verorten:

1. Dimension der Erzählerschaft: Eine Erzählung wird von einem/r Erzähler/in oder von einer multiplen Erzählerschaft realisiert.

2. Dimension der Erzählwürdigkeit: Eine Geschichte ist offenkundig wert erzählt zu werden oder scheinbar irrelevant.

3. Dimension der Einbettung der Geschichte in ihren Kontext: Eine Geschichte ist in ihren Kontext eingebettet oder von ihm gelöst.

4. Dimension der Linearität der erzählten Ereignisse: Eine Geschichte ist zeitlich geschlossen, offen oder multilinear.

5. Dimension der Wertung (= moralische Haltung der Erzähler/inen zu dem Ereignis): Die Einstellung der Erzähler/inen zu den erzählten Ereignissen ist deutlich oder verdeckt.

Ordnet man (bimodale) Kompakterzählungen skalar innerhalb dieser Dimensionen an, ergeben sich - indiziert mittels der Kreuze - folgende narrationsspezfische Merkmale sie (Abb. 9.18):

Grundsätzlich handelt es sich um inhaltlich abgeschlossene Erzählungen, die auf einem linearen Ablauf der erzählten Ereignisse beruhen. Das Augenmerk für spezifizierende Merkmale von Kompakterzählungen liegt vor allem auf den erzähltheoretischen Merkmalen: Erzählwürdigkeit, Einbettung 


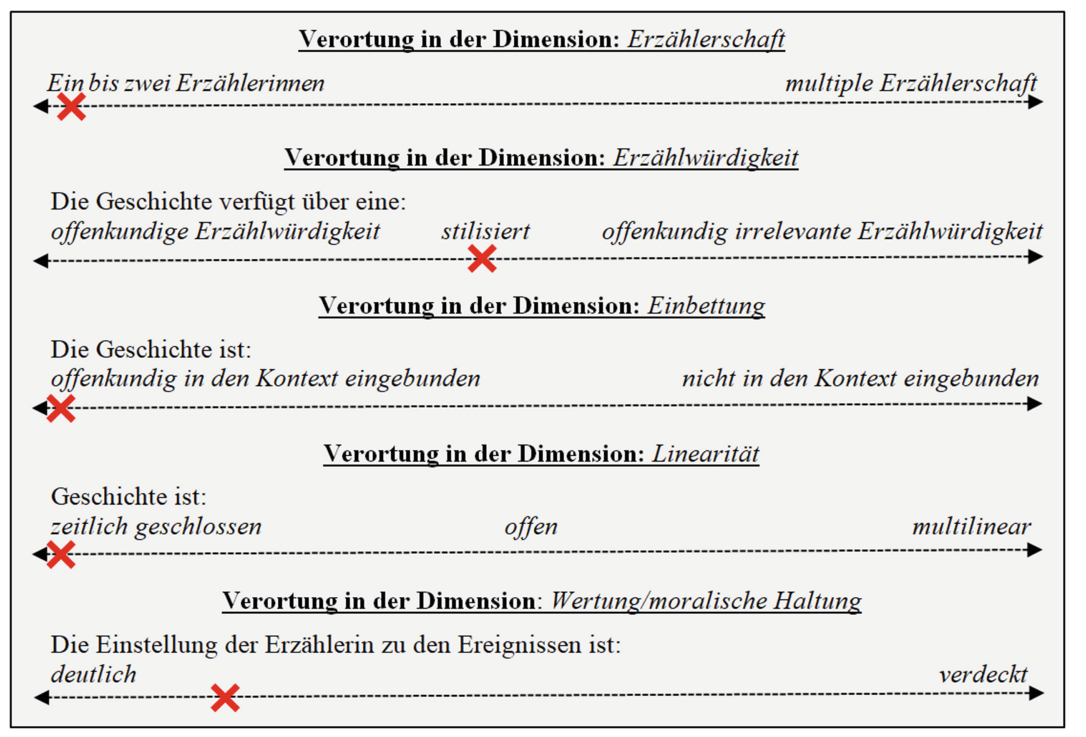

Abb.9.18 Tendenzen von Kompakterzählungen innerhalb der narrativen Dimensionen für Erzählungen im Internet (angelehnt an Ochs/Capps 2001: 22 ff., nach Page 2015: 333)

der Geschichte in den Kontext, Wertung. Die Erzählwürdigkeit von unternehmensbezogenen Kompakterzählungen begründet sich zum einen in deren Veröffentlichung auf der Internetplattform, zum anderen in der Stilisierung durch unverfängliche Komplikationen, in der Bewertung erzählter Ereignisse durch die Erzähler/innen selbst sowie anhand von Evaluationen der Protagonist/innen, die die Erzähler/innen wiedergeben. Es besteht demnach eine enge Verbindung mit der Wertung/moralischen Haltung, die wichtige Elemente zur imagefördernden $(\mathrm{S}) \mathrm{D}$ von Unternehmen trägt: Die positiven Inhalte der Geschichte sind immer in den Unternehmenskontext eingebettet. Entsprechend handelt es sich bei diesen Kompakterzählungen im Gegensatz zu Geschichten von Privatpersonen im Internet um reflektiertes, bewusst geplantes und strategisch ausgerichtetes Erzählen mit Funktion. Es existieren in der Dimension der Erzählwürdigkeit Kompakterzählungen, die thematisch von außergewöhnlichen Ereignissen (z. B. die Geschichte von Eule Olli, dem „ersten“ Strafzettel, das Making-Of zu einer Mitarbeiterkampagne u. a.) oder berufsspezifischen Tätigkeiten im Unternehmen (z. B. Werdegang von Mitarbeiter/innen oder Erfahrungen von Praktikant/innen und Studierenden) handeln. 
Bezogen auf die Erzählerschaft und die Linearität unterscheiden sich diese Kompakterzählungen nicht grundlegend von prototypischen Erzählungen: Es treten Ich-Erzähler/innen, oder - deutlich häufiger - Erzähler/innen in der 3. Person Singular auf, die über andere schreiben. Anders als in diskontinuierlichen Erzählungen besteht in Kompakterzählungen keine kollaborative Erzählerschaft. Der/Die Erzähler/in lässt ihre Protagonist/innen allerdings zu Wort kommen, wodurch sich die Erzählperspektive partiell verschiebt (ähnlich eines „MiniaturFeatures"). Für gewöhnlich sind die untersuchten Kompakterzählungen in der chronologischen Ereignisabfolge linear angelegt und thematisch geschlossen, auch wenn für Erzählungen untypische Sprechhandlungen wie Lob und Dank z. B. an Akteure und Protagonist/innen aus der Erzählung - oder Fotografien die uRE beenden.

\subsection{Diskontinuierliche Erzählungen auf Twitter}

Neben Kompakterzählungen besteht eine zweite Realisierungsvariante von Small Stories im Unternehmenskontext, die ich unter dem Arbeitsbegriff diskontinuierliche Erzählungen innerhalb von uRE zusammenfasse. Das spezifizierende Adjektiv ,diskontinuierlich ' bezieht sich darauf, dass die Abfolge einzelner Elemente der Erzählung unterbrochen ist, in unregelmäßigen Abständen fortgesetzt wird und in einigen Fällen nicht linear dem Ereignisverlauf folgt. Das geschieht in den Daten überwiegend dadurch, dass von der Geschichte unabhängige Posts zwischen die Erzählelemente/-fragmente einer Geschichte auf die Veröffentlichungsplattform gestellt werden. Ebenso wie Kompakterzählungen werden diskontinuierlichen Erzählungen Fotografien und darüber hinaus Bewegtbilder beigefügt.

Das folgende Beispiel illustriert, wie eine Gesamterzählung des narrativen Vermittlungsvorkommens auf der Internetplattform Twitter auftreten kann: Die Geschichte handelt von einem sozialen Projekt des Energieunternehmens Energie Baden-Württemberg (EnBW) - dem EnBW-Macher-Bus ${ }^{51}$. Interessierte können sich über die EnBW-Webseite für einen Einsatz des EnBW-Macher-Busses bewerben. Gewinnen sie, führen von der EnBW freigestellte Mitarbeiter/innen gemeinsam mit ihnen ein soziales Teilprojekt durch. Zusätzlich sponsert die EnBW für das Unterfangen einen festgelegten Betrag. Die Projektumsetzung wird im Verlauf und abschließend für verschiedene Internetplattformen aufbereitet. Die folgende diskontinuierliche Erzählung handelt davon, dass der EnBW-Macher-Bus im Ort St. Märgen eine Mountainbikestrecke für die Kinder des ortsansässigen Ski-Clubs anlegt (EnBW, Twitter, 2016-07-29; 2016-07-30. Korpusquelle: EnBW_720.3_TW):

${ }^{51}$ Die EnBW wiederholt die Aktion min. einmal jährlich, zuletzt 2019. 
(Bsp.) Diskontinuierliches Erzählen auf Twitter: Der Macherbus der EnBW 2016 in St. Märgen

1) Anfang: Die Erzählung beginnt mit dem ersten Tweet über das Projekt „Moutainbikestrecke für Kinder":

$$
\text { Gow EnBW } 0
$$

$$
\text { 2. Folgen }
$$

Jetzt geht's los, wir packen an: \#EnBW \#MacherBus baut Mountainbike-Strecke in St. Märgen. \#WirMachenDasSchon

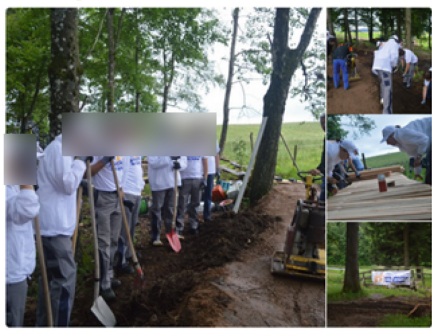

1: Beginn der diskontinuierlichen Erzählung ,EnBWMacher-Bus in St. Märgen “

3) Mitte/Schluss: Im dritten Tweet wird das Ergebnis der Aktion benannt und fotografisch sowohl mit Bildern aus der Ereignisrepräsentation als auch des Endprodukts dokomentiert:

$$
\rightarrow \text { EnBW } 0
$$$$
\text { 2. Folgen }
$$

St. Märgen hat eine neue MountainbikeStrecke, dank fleißigen Helfern \& dem \#EnBW \#MacherBus \#WirMachenDasSchon

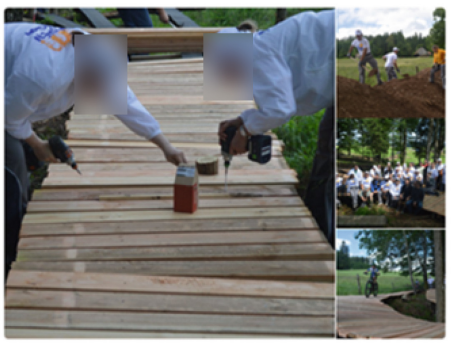

3: Mittelteil 2/2 der diskontinuierlichen Erzählung ,EnBW-Macher-Bus in St. Märgen “
2) Mitte: In den zweiten Tweet ist ein YouTube-Video eingebunden, das die Ereignisrepräsentation von Anfang bis Ende filmisch vorführt:

$$
\text { - nov EnBW } 0
$$$$
\text { 2. Folgen }
$$

Das war der \#EnBW \#MacherBus-Einsatz beim Ski-Club St. Märgen: youtu.be /yGFhTkpzHXw \#WirMachenDasSchon \#Mountainbike

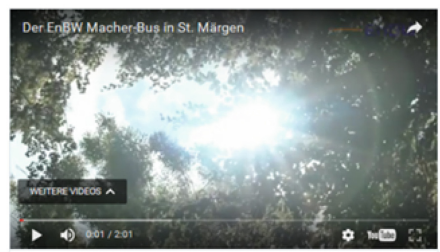

2: Mittelteil 1/2 der diskontinuierlichen Erzählung „EnBW-Macher-Bus in St. Märgen“"

4) Schluss: Im letzten Tweet ist das Ergebnis abschlieBend textuell und audiovisuell dargeboten. Das eingefügte Bewegtbild visualisiert die Bewertung der Aktions-Gewinner anhand einer Laola-Welle und anhand von Jubelrufen und Gelächter:

cos EnBW

2. folgen

\#EnBW \#MacherBus in St. Märgen: die neue Mountainbike-Strecke hält was Sie verspricht! \#WirMachenDasSchon

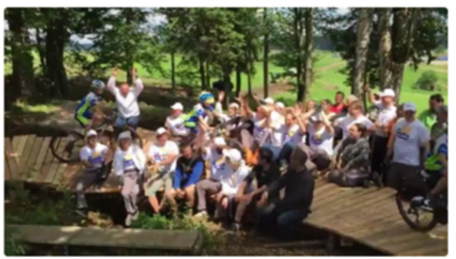

4: Ende der diskontinuierlichen Erzählung ,EnBWMacher-Bus in St. Märgen " 
Das Beispiel verdeutlicht, dass die Erzählelemente fragmentarisch zu verschiedenen Zeitpunkten veröffentlicht werden. Es gibt einen Anfang, eine Mitte und einen Schluss, die multimodal präsentiert und von anderen Beiträgen auf der EnBW-Twitter-Seite unterbrochen werden. Ein weiteres Merkmal von diskontinuierlichen Erzählungen ist, dass die Erzählelemente bzw. -fragmente in unterschiedlichem Grad ausgebaut sind (Text, Text/Bewegtbild, Text/Bild). In dem obigen Beispiel sind die einzelnen Posts sprachlich so gestaltet, dass der Eindruck entsteht, sie würden fortlaufend aus dem laufenden Projekt heraus veröffentlicht. Dieser Eindruck von Unmittelbarkeit entsteht insbesondere durch die Präsensverwendung: Los geht's, wir packen an, St. Märgen hat eine neue Mountainbikestrecke, die neue Mountainbikestecke hält was sie verspricht. Der Text kontextualisiert die Bilder und den Film, die neben Tätigkeiten und Vorgängen auch das Ergebnis vorführen. Dadurch werten sie den Text semantisch auf und tragen einen erheblichen Anteil an den Vermittlungsinhalten der Geschichte. Im zweiten Post bricht der Text jedoch mit der suggerierten Unmittelbarkeit, da er im Präteritum das war der EnBW MacherBus Einsatz auf den eingebundenen YouTube-Film referiert: Insbesondere da es sich um einen professionell erstellten Film handelt, den man eben nicht nebenher produzieren kann. Das Einbinden professionell erstellter YouTube-Filme in einzelne Posts ist bezeichnend für diskontinuierliche Erzählungen im Unternehmenskontext.

\subsubsection{Hintergrundinformationen zum Erzählen via Twitter}

Zwar weist Twitter im Vergleich mit Facebook Ähnlichkeiten auf, unterscheidet sich mit Blick auf diskontinuierliche Erzählungen jedoch vor allem in einem Punkt von Facebook: Die Veröffentlichung der Nachrichten erfolgt wesentlich schneller. Daraus folgt für Autor/innen und Autorenkollektive, dass eine Nachricht nach einer deutlich kürzeren Zeitspanne bereits ,veraltet“ (Primbs 2016: 30) ist $^{52}$. Beiträge auf Twitter unterliegen einer engen Zeichenrestriktion (140 Zeichen pro Nachricht) und sind für den öffentlichen Kontext gedacht. Der Tweet wird automatisch für all jene sichtbar, die dem/der Verfasser/in folgen (Follower). Hat ein/e Twitter-Nutzer/in viele Follower/innen, haben ihre Tweets eine hohe Reichweite. Das erklärt u. a. das Interesse von Unternehmen an der Internetplattform. Innerhalb der Textnachrichten haben sich eigene Konventionen in Form

\footnotetext{
52 Primbs setzt bei Twitter eine zeitliche Verzögerung von ca. 30 Minuten an, während es bei Facebook bis zu sechs Stunden sein können (ebd. 2016: 31).
} 
einer Twitter-Syntax herausgebildet, die in einer narratologisch motivierten Untersuchung beachtet werden müssen. Dazu gehört u. a. (1) das @-Präfix vor dem Nutzernamen. Es kann an unterschiedlichen Stellen in einem Tweet auftreten. Am Beginn eines Tweets weist das Zeichen diesen als eine Antwort (Reply) aus. (2) Eine weitere Konvention ist die Abkürzung „RT(@twittername: [Zitierter Tweet])“, die anzeigt, dass ein Tweet von einem/r anderen Nutzer/in erneut veröffentlicht wurde. (3) Verändert man einen zitierten Original-Tweet, zeigt das die Buchstabenfolge „MT(@twittername: [Modifizierter Tweet]“ an. Für Unternehmen ist ein sehr wichtiger Bestandteil der Twitter-Syntax (4) der Hashtag ${ }^{53}$. Ein Hashtag „macht aus einer danach folgenden Zeichenfolge ein Schlagwort“ (Primbs 2016: 31). Markiert wird das mittels der vorangestellten Raute (,\#“) ${ }^{54}$. Der Vorteil eines Hashtag ist, dass jedem, der ihn in die Twitter-Suche eingibt, damit verschlagwortete Nachrichten ausgegeben werden. Somit ist ein Hashtag eine hoch effiziente Ressource, Tweets sichtbar zu machen (siehe hierzu auch Page 2012, 2014; Scott 2015). Zusätzlich wird er, sobald man auf den tag klickt zu einem Hyperlink und erleichtert den Rezipient/innen zusammengehörende Inhalte inmitten des „Rauschens“ von Twitter zu finden (Page 2014).

Es sind aber nicht nur formale Merkmale von Twitter, die sich auf das Erzählen auswirken (Zeichenbeschränkung, Veröffentlichungszeitraum, Konventionen, Einbezug von Bildern und Bewegtbildern), auch inhaltliche Merkmale prägen den Realisierungstypen. Das betrifft die Ausgangsfrage, zu deren Beantwortung Twitter in der Nutzermaske explizit auffordert: Was gibt's Neues? Ausgehend von dieser Frage entstehen Ereignisketten, deren Zusammenhang Rezipient/innen durch das Thema und anhand der automatisch vergebenen Veröffentlichungs-Zeitmarke selbst erschließen müssen. Die eigentliche Ereignisdarstellung beeinflusst das insofern, als die Ereignisse im unmarkierten Fall nur selten retrospektiv angelegt sind (im Gegenteil zu Kompakterzählungen). An die Möglichkeit in Quasi-Echtzeit etwas mitzuteilen, knüpft ein weiteres inhaltliches Merkmal an, das für Posts im öffentlichen Kontext aus der journalistischen Perspektive wie folgt benannt wird: Die Inhalte von Tweets beziehen sich stärker auf „News und Pointen“ (Primbs 2016: 37) - im Vordergrund steht die Informationsweitergabe -, als auf Emotionen und Meinungen. In diesem Zusammenhang kann Pages Resümee über Twittererzählungen von Prominenten gesehen werden:

\footnotetext{
${ }^{53}$ Hashtags sind in ihrem Ursprung eine Erneuerung der Twitternutzer/innen. Ihre Vorläufer waren sogenannte „Channel-Tags“ (Scott 2015: 9), die in ihrer Funktionalität erweitert wurden und seit 2009 offiziell als Merkmal der Webseite angenommen wurden.

${ }^{54}$ Mittlerweile sind Hashtags auch auf Facebook, Google + und Instagram gebräuchlich (Primbs 2016: 34).
} 
,the news-sharing function of twitter where broadcasting information is more important than generating social connectedness.“ (Page 2013: 107, Hervorhebung U.A.)

Das vom Journalismus benannte Merkmal und Pages Beobachtung treffen ebenfalls auf Twitter-Erzählungen von Unternehmen zu, was sich bereits in den Themenfeldern widerspiegelt, aber auch in der unidirektionalen Kommunikationsrichtung ${ }^{55}$. Die erhobenen diskontinuierlichen Erzählungen von Unternehmen sind in erster Linie nicht darauf angelegt, Interaktivität mit der Zielgruppe herzustellen. Twitter-Erzählungen von Privatpersonen zeichnen sich nach Puschmann/Heyd (2012) dadurch aus, dass sie sich

„durch ihre zeitliche Nähe zum Erzählten, durch ihr Beschreibungsobjekt (zumeist der Erzählende selbst), durch den Grad ihrer Strukturierung (eher gering) und durch ihre Planungsintensität (ebenfalls eher gering) [unterscheiden]“ (ebd. 2012: $184 \mathrm{f}$.).

Da es sich bei diskontinuierlichen Erzählungen von Unternehmen um Maßnahmen innerhalb der strategischen Unternehmenskommunikation handelt, gehe ich davon aus, dass zwar Unternehmen an diese Nutzererwartungen anknüpfen, die im Jahr 2020 (also vierzehn Jahre nach der Freigabe von Twitter für die Allgemeinheit) schon konventionalisiert sein dürften, doch werden die diskontinuierlichen Erzählungen aufgrund der strategischen Anwendung auch Unterschiede aufweisen. Zu erwarten ist außerdem, dass der Planungsgrad zwar vordergründig gering erscheinen mag, hintergründig jedoch funktional und somit durchdachter ist, als das den Rezipient/innen auf den ersten Blick ersichtlich ist. Der wissenschaftliche Beitrag dieser Arbeit liegt demnach darin, dass der Forschungsstand über Small Stories von privaten Nutzer/innen um narrative Praktiken im institutionellen Rahmen erweitert wird. Gleichzeitig verdeutlicht das, dass es sich gerade nicht um alltägliches Erzählen handelt, sondern daran aus strategischen Erwägungen angeknüpft wird.

\footnotetext{
${ }^{55}$ Die deutliche Fokussierung auf Informationen, Unterhaltung in Verbindung mit der öffentlichen, meist unidirektionalen, also nicht interpersonellen Kommunikation, ist ursächlich dafür, dass Twitter mediengeschichtlich nicht zu den üblichen Repräsentanten der sozialen Medien gezählt wird (Puschmann/Heyd 2012: 173).
} 


\subsubsection{Datengrundlage und konstitutive Themenfelder für diskontinuierliche Erzählungen}

Der Befund, dass Unternehmen diskontinuierliche Erzählungen im Internet realisieren, kristallisierte sich im Verlauf der Datensortierung und -archivierung heraus. Die Herausforderung bestand insbesondere im Fall der Internetplattformen Twitter und Facebook darin, jene Elemente auszumachen, die zu einer diskontinuierlichen Erzählung gehören. Für das sekundäre Subkorpus habe ich aus den primären Subkorpora jene diskontinuierlichen Erzählungen ausgewählt, die den Realisierungstypen am deutlichsten abbilden. Das hat zwar die Quantität der Daten reduziert, ist $\mathrm{m}$. E. für das qualitative Vorgehen jedoch vertretbar. In der untenstehenden Tabelle sind die gesammelten diskontinuierlichen Erzählungen mit ihren jeweiligen Internetplattformen aufgeführt (primäres Subkorpus). Die Zeile ,Anzahl der Erzählelemente/-fragmente" verdeutlicht, dass sich die Gesamterzählung aus mehreren Teilen zusammenfügt (Tab. 9.3).

Tab.9.3 Absolute Anzahl der Daten des Realisierungstypen ,diskontinuierliche Erzählungen` im Datenkorpus (= primäres Subkorpus)

\begin{tabular}{l|l|l|l|l|l|l}
\hline Realisierungsplattform & Twitter & YouTube & Weblog & Webseite & Facebook & Gesamt \\
\hline $\begin{array}{l}\text { Anzahl der } \\
\text { Erzählelementel } \\
\text {-fragmente }\end{array}$ & 132 & 5 & 3 & 19 & 13 & N = 172 \\
\hline $\begin{array}{l}\text { Anzahl der } \\
\text { zusammengeführten } \\
\text { Erzählungen }\end{array}$ & 11 & 1 & 1 & 2 & 2 & N = 17 \\
\hline Betroffene Unternehmen & $\begin{array}{l}\text { VW } \\
\text { E.ON } \\
\text { EnBW } \\
\text { Rewe } \\
\text { Telefonica } \\
\text { Dtl. } \\
\text { Dt. } \\
\text { Telekom }\end{array}$ & Telekom & Telekom & Bosch & $\begin{array}{l}\text { Rewe } \\
\text { Telekom } \\
\text { EnBW }\end{array}$ & \\
\hline
\end{tabular}

Zusätzlich wird in der tabellarischen Auflistung ersichtlich, dass in dem primären Subkorpus die meisten diskontinuierlichen Erzählungen auf der Internetplattform Twitter auftreten. Somit scheint sich Twitter am ehesten für die Umsetzung des Realisierungstypen zu eignen. Weiter zeigt sich, dass das Vorkommen nicht an eine Branche gebunden ist: Die Daten entstammen allen fünf 
erhobenen Branchen (VW / E.ON, EnBW / Rewe / Telefonica Deutschland, Deutsche Telekom / Siemens, Bosch).

Die Analyse der Themenfelder in den untersuchten diskontinuierlichen Erzählungen zeigt in Anbetracht des kleine Analysekorpus eine Tendenz an, welche Themenfelder für den Realisierungstypen narrativ aufbereitet werden. Mit ca. 65 Prozent ( $\mathrm{N}=11$ von 17) sind die meisten diskontinuierlichen Erzählungen auf der Internetplattform Twitter dokumentiert. Kompakterzählungen wurden dagegen vermehrt auf der Internetplattform Facebook lokalisiert. Bezüglich der Themenfelder ist in bimodalen Kompakterzählungen das Themenfeld rund um ,Ausbildung und Arbeitsplatz' mit ca. 63 Prozent stark vertreten, während in den erhobenen diskontinuierlichen Erzählungen lediglich ca. 12 Prozent darauf entfallen. Dagegen ist in diskontinuierlichen Erzählungen das Themenfeld ,aktuelle Informationen und Öffentlichkeitsarbeit" mit 82 Prozent stark vertreten, das umgekehrt in bimodalen Kompakterzählungen in 23 Prozent der erhobenen Daten vorkommt. In erster Linie indiziert der Vergleich, dass sich die Daten bezüglich ihrer Themenfelder im Anwendungskontext entgegengesetzt präsentieren. Auf Basis der deskriptiven Auswertung lassen sich nur Hypothesen ableiten. Dennoch legt der Vergleich nahe, dass innerhalb des Korpus Unternehmen für ihre (S)D bestimmte Themenfelder bevorzugen und damit verbunden entweder die Internetplattform Twitter oder Facebook wählen.

\subsubsection{Erzählerbeteiligung: Grade der Involviertheit}

Aufgrund des spezifizierenden Merkmals diskontinuierlicher Erzählungen, wonach sich die Geschichte durch Erzählelemente/-fragmente zusammensetzt und Bilder sowie Bewegtbilder integriert sein können, lassen hinsichtlich der Erzählerbeteiligung sich bedingende Situationen unterscheiden:

a) Auf einer Plattform können mehrere Erzähler/innen an einer Geschichte mitwirken. Dadurch variieren jedoch auch die Erzählperspektiven.

b) Die Erzähler/innen in einer diskontinuierlichen Erzählung sind in unterschiedlichem Maße in die Geschehnisse involviert.

Dies führt dazu, dass die Erzähler/innen und ihre Perspektive wie auch ihre Beteiligung am Geschehen für jedes Erzählelement/-fragment neu bewertet werden müssen. Für Erzählungen von Unternehmen - insbesondere in den sozialen Medien - ist das relevant, da mit der Involviertheit der Erzähler/innen ihr Authentizitätsanspruch steigt und damit verbunden die Glaubwürdigkeit der 
kommunizierten Inhalte. Die Erzählperspektive und die damit verbundene Involviertheit der Erzähler/innen ist in diskontinuierlichen Erzählungen nicht nur von sprachlichen Merkmalen abhängig. Dabei spielen ebenso Bilder und Bewegtbilder eine Rolle. So können (Bewegt-)Bilder eine Beteiligung am Geschehen implizieren, die der Erzähltext entweder stützt oder offen lässt. Ein Foto eröffnet selbst eine Perspektive auf das Geschehen und/oder kann die Personen aus der Geschichte - einschließlich der Erzähler/innen - abbilden. So können mit oder ohne sprachliche Bezugnahme unterschiedliche Grade der Involviertheit der Erzähler/innen an dem Geschehen suggeriert werden. Um eine graduelle Abstufung der Erzählerbeteiligung zu erfassen, bietet Lanser (nach Martinez/Scheffel 2007: 82) einen literaturwissenschaftlichen Ansatz (siehe die Abb. unten). Mithilfe dieses Ansatzes lässt sich auch der Grad der Erzählerbeteiligung in diskontinuierlichen Erzählungen nachverfolgen. Dabei zeigt sich, inwiefern (Bewegt-)Bilder auf die Erzählerbeteiligung Einfluss nehmen (Abb. 9.19).

\begin{tabular}{|c|c|c|}
\hline $\begin{array}{l}\text { Heterodiegetisch } \\
\text { (3. Person/keine Figur der erzählten Welt) }\end{array}$ & & $\begin{array}{r}\text { Homodiegetisch } \\
\text { (1. Person/Figur der erzählten Welt) }\end{array}$ \\
\hline 1 & 2 & 5 \\
\hline Unbeteiligte/r Erzähler/in| & $\begin{array}{l}2: \\
3: \\
4: \\
5: \\
6:\end{array}$ & $\begin{array}{l}\text { unbeteiligte/r Beobachter/in } \\
\text { beteiligte/r Beobachter/in } \\
\text { Nebenfigur } \\
\text { eine der Hauptfiguren } \\
\text { die Hauptfigur (= autodiegetisch) }\end{array}$ \\
\hline
\end{tabular}

Abb.9.19 Unterscheidung der Beteiligung von heterodiegetischen und homodiegetischen Erzähler/innen (nach Lanser, aus Martinez/Scheffel 2007: 82)

Den Erzählerwechsel in diskontinuierlichen Erzählungen müssen Rezipient/innen meist den Kontextinformationen (kleine Abbildungen der Verfassenden oberhalb der Posts oder Nutzernamen) entnehmen. (1) Unter heterodiegetisch ausgerichteten - also unbeteiligten Erzähler/innen - sind jene zu verstehen, die selbst keine Figur in der erzählten Welt sind. Meist geschehen Veröffentlichungen mit heterodiegetischen Erzähler/innen unter dem Label der Firma und nicht unter einem individuellen Nutzernamen: beispielsweise in dem folgenden Erzählfragment der diskontinuierlichen Erzählung von Telefonica Dtl. auf Twitter über die Wanderfalken ,Frida \& Fridolin' (siehe die Markierung, Bsp.: nicht personifizierter Tweet (Telefonica Deutschland, Twitter, 2015-03-25. Korpusquelle: Telef_782.2_TW): 
Telefónica Germany @telefonica_de - 25. März 2015

Tateriaa Frida \& Fridolin sind zurück:

Wanderfalken brüten wieder auf o2

\#LTE-Mast j.mp/19kJMd6 \#Webcam

sendet 24/7 live vom Nest (MG)

Ein/e persönliche/r Erzähler/in ist in dem Post nicht auszumachen, da lediglich der Firmenname als Absender/in aufgeführt wird (siehe die Unterstreichung). Das Erzählfragment beginnt mit der Namensnennung der beiden Protagonist/innen ,Frida‘ und ,Fridolin'. Das Fragment eröffnet die diskontinuierliche Erzählung mit dem Ereignis (= Themahinweis), dass zwei Wanderfalken zum Brüten auf einen LTE-Mast von Telefonica Dtl. zurückgekehrt sind. Auf die gleiche Art verfährt der/die Erzähler/in in den folgenden Posts, indem sie mit der Stimme eines/r unbeteiligten Erzähler/in (Benennungen der Protagonist/innen: Wanderfalken, Schneebälle, Küken) das weitere Geschehen kundtut. Der/die Erzähler/in gibt also keinen sprachlichen oder bildlichen Hinweis darauf, inwiefern sie an dem Geschehen teilhat. (2) Unbeteiligte Beobachter/innen sind ebenfalls meist „,anonyme“ Personen, die ,unter dem Deckmantel“ des Unternehmens eine Beitrag verfassen. Das ist in dem obigen Beispiel zwar auch der Fall, doch weißt nichts in dem Beitrag darauf hin, dass der/die Erzähler/in aus der Position des/der Beobachter/in heraus erzählt. Im Gegensatz dazu verdeutlich die nächste Abbildung von Volkswagen, wie ein/e Erzähler/in als Beobachter/in in Erscheinung tritt. Es handelt sich um den Höhepunkt der Gesamterzählung über die Eröffnung des VW Group Forums ,Drive“ (Abb. 9.20):

In dem Beispiel postet ein/e am eigentlichen Geschehen unbeteiligte/r Beobachter/in den Höhepunkt der Gesamterzählung: Auftritt des britischen Sängers Robbie Williams. Dass hier von einem/r homodiegetischen Erzähler/in ausgegangen werden kann (!), hat mit der Unmittelbarkeit der Internetplattform und der filmischen Perspektive zu tun. Der Twittertext für sich allein genommen lässt sowohl die Option eines/r homo- wie auch eines/r heterodiegetischen Erzähler/in zu. Es wird lediglich auf den eingebundenen YouTube Film verwiesen, in dem der Musiker zu sehen ist. Die filmische Perspektive vermittelt jedoch den Eindruck, dass wir durch die Kameralinse des/der Erzähler/in an dem Geschehen in gleicher Weise wie sie selbst teilhaben: nämlich aus der Position eines/r Beobachter/in. Unterstützt wird dieser Eindruck darüber hinaus durch den improvisiert wirkenden Filmausschnitt (verrüttelte Kameraführung, Personen, die in das Bild laufen, viele Hintergrundgeräusche u. a.). Davon ausgehend können wir den Text als 

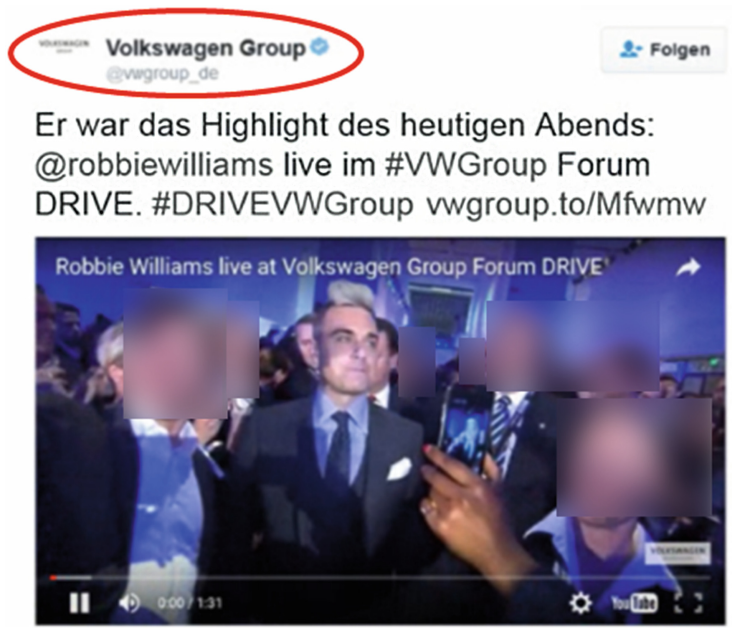

Abb. 9.20 Tweet eines/r homodiegetisch unbeteiligten Beobachter/in (VW, Twitter, 201504-28. Korpusquelle: VW_455_TW)

homodiegetischen Kommentar zu dem Ereignis bewerten. (3) Beteiligte Beobachter/innen weisen sich dagegen als einen Teil der erzählten Welt aus. Sie erzählen jedoch, ohne zu den Protagonist/innen der eigentlichen Geschichte zu gehören. In der nächsten Abbildung eines Tweets von Telefonica Dtl. stellt sich das gesamte Team für eine Gesamterzählung über die Eröffnung eines O2 Fan Events vor (Abb. 9.21):

Das inkludierende, wir' sowie die direkte Adressierung Wir freuen uns auf $\underline{\text { Euch }}$ vermitteln den Eindruck, dass diejenigen, die den Twitterkanal bespielen, als Teil der Unternehmensgemeinschaft an der Veranstaltung beteiligt sind. Sie treten aber nicht als Protagonist/innen in der Erzählung in Erscheinung, sondern dokumentieren das Event aus ihrer Außensicht. Sichtbar werden sie in der Verbindung des Personalpronomens wir mit der Fotografie. (4) Die Beteiligung von homodiegetischen Erzähler/innen nimmt zu, wenn sie nicht nur in Form von Beobachter/innen, sondern als Nebenfiguren erzählen. In diesem Fall sind sie „nur“ am Rande der erzählten Handlung beteiligt (Martinez/Scheffel 2007: 82). Ein Beispiel dafür ist die auf Twitter veröffentlichte mündliche Erzählung (eingebundenes Bewegtbild) von der Telekom Netz Tour 2016 mit dem Titel Drama auf dem Feldberg. Inhaltlich geht es darum, dass als Höhepunkt der Tour ein 
Telefónica Germany hat retweeter

o2 Entdecken @o2entdecken - 17. Dez 2014

Wir freuen uns auf Euch:Heute um 17h fällt der Startschuss zum ersten \#02FanEvent in der @o2WorldHamburg. \#Vorfreude

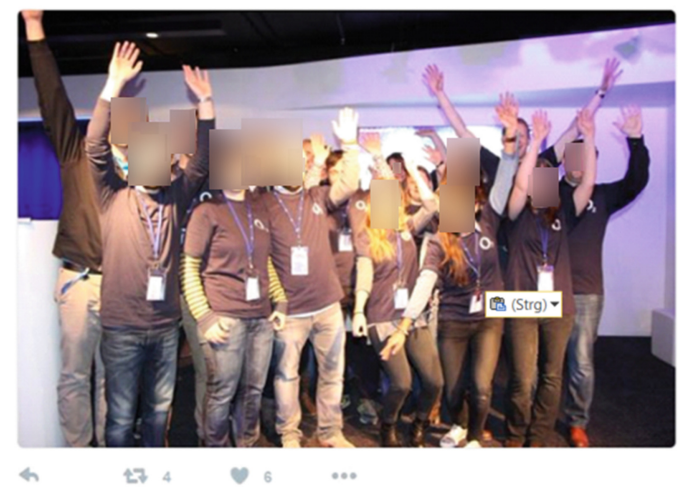

Abb.9.21 Erzähler/in als beteiligte/r Beobachter/in (Telefonica Deutschland, Twitter, 2014-12-17. Korpusquelle: Telef_786_TW)

Radrennen auf den Feldberg zwischen zwei Protagonisten stattfand. In der Erzählung präsentiert sich der Erzähler als Nebenfigur, indem er rückblickend von seinen Eindrücken von dem Abenteuer der anderen beiden Protagonisten in der Geschichte erzählt. Im Mittelpunkt steht somit nicht das Handeln des Erzählers, sondern das der zwei Kollegen: 
(Bsp.) Beteiligung homodiegetische/r Erzähler/in, Nebenfigur

(Telekom, Twitter, 2016-12-27. Korpusquelle: DTKOM_847.2.1_TW)

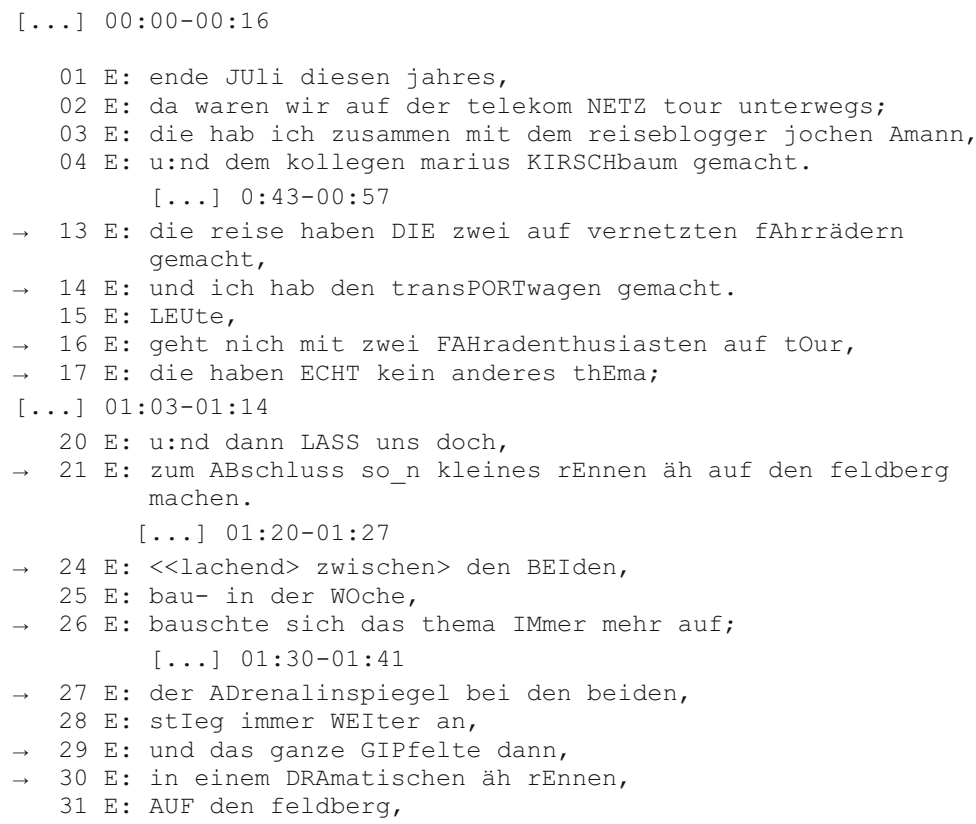

In Zeile 14 des Transkripts über das Drama auf dem Feldberg benennt der Sprecher seine Rolle und ich hab den transPORTwagen gemacht, während er innerhalb dieser Erzählung seine zwei Begleiter als Protagonisten des Radrennens ausweist: a) aufgrund inhaltlicher Informationen (Z. 13, 21, 24, 26, 27, 29, 30), b) aufgrund einer Charakterisierung (Z. 16, 17) und c) letztlich mittels des Pronomens beide (Z. 24, 27), mit dem er sich selbst exkludiert. (5) Ist ein/e Erzähler/in die Hauptfigur der Erzählung - somit unmittelbar betroffen von dem Ereignis nimmt das Maß seiner/ihrer Beteiligung als homodiegetische/r Erzähler/in zu. In einem Erzählelement eines Corporate Blog-Eintrags über einen anderen Abschnitt der selben Tour stellt sich ein Erzähler als einen der Handlungsträger vor (fett hervorgehoben): 
Start der Tour: Erste Station Glasfaserausbau

Heute gegen halb zehn fiel in Kenzingen bei Emmendingen der Startschuss für den mindestens 230 Kilometer langen Trip durch den Südwestens [sic] Baden- Württemberg [sic]. Ich schreibe mindestens, denn Voraussetzung dafür ist, dass wir die besten Wege auf Anhieb finden. Mit dabei sind mein Telekom-Kollege [Sven Hold] und der Blogger [Jochen Amann]. Eine Woche werden wir unterwegs sein. (Telekom, Corporate Blog, 2016-07-25. Korpusquelle: DTKOM_847.3_WB)

Auch hier zeigt das inkludierende, wir' die gemeinschaftliche Beteiligung an dem geplanten Unterfangen. Spätestens durch die Präpositionalphrase mit dabei sind mein Telekom-Kollege [...] und der Blogger [...] verdeutlicht der Erzähler, an dem erzählten Geschehen unmittelbar als Hauptakteur beteiligt zu sein. Dadurch erkennen die Rezipient/innen, dass er nicht nur seine eigene persönliche Geschichte, sondern die des gesamten Tourteams erzählt. (6) Ein/e homodiegetische/r Ich-Erzähler/in, der/die ihre persönliche Geschichte erzählt, ist in den erhobenen diskontinuierlichen Erzählungen beispielsweise auch auf eingebundenen YouTube Ausschnitten zu finden. In so einer YouTube-Erzählung berichtet einer der Telekom-Netz-Tour-Teilnehmer von den Erlebnissen an den einzelnen Tourtagen. Der erste Tag beginnt mit folgender Einleitung (Telekom, YouTube, 2016-07-25, „Telekom Netz Tour 2016, Tag 1. Korpusquelle: DTKOM_847.4_YT):

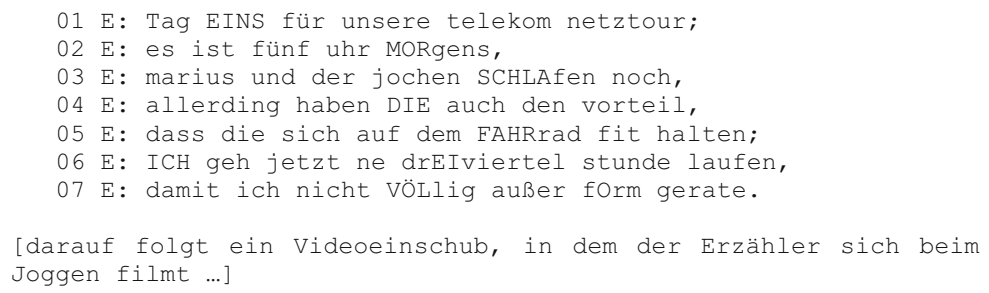

Der Erzähler erzählt in einer Art Videotagebuch von seinen Erlebnissen am ersten Tag der Telekom Netz Tour 2016.

Die Beispiele verdeutlichen, dass die multimodale Präsentation den Grad der Beteiligung von Erzähler/innen an dem Erzählten beeinflusst. Sprachliche Indikatoren verbunden mit Fotografien und/oder Bewegtbildern suggerieren unterschiedliche Abstufungen der Involviertheit von Erzähler/innen, was im Kontext der sozialen Medien Bedeutung hat. 


\subsubsection{Narrative Themenentfaltung diskontinuierlicher Erzählungen}

Die narrative Themenentfaltung entwickelt sich in diskontinuierlichen Erzählungen nicht innerhalb eines Textgefüges, sondern in lose verbundenen Erzählfragmenten. Hausendorf et al. (2019: 171) sprechen daher von einer komplexen „Textsammlung“ mit relativ autonomen Teiltexten/Tweets. Da in diskontinuierlichen Erzählung bestimmte Teile der Textsammlung auf gemeinsame Inhalte referieren, münden sie in dieses spezifische narrative Vermittlungsvorkommen. Die chronologische Abfolge der Elemente/Fragmente ist unbeständig. Sie orientiert sich jedoch daran, dass etwas möglichst zeitnah an einem Geschehen getwittert wird. Hinzu kommt, dass Unternehmen geplant kommunizieren. Daraus folgt, dass die Erzählungen nicht unorganisiert in der virtuellen Gleichzeitigkeit der Internetplattform auftreten. Allerdings handelt es sich bei ihrer Entwicklung nur bedingt um das, was in der Textlinguistik unter einer narrativen Themenentfaltung zu verstehen ist. Da zumindest der Eindruck vermittelt werden soll, dass die uRE im Moment des Geschehens getwittert wird, gehe ich von einer weitgehend „,natürlichen“ Strukturierung aus. Sie spiegelt sich in der inhaltsbezogenen Dreiteilung der Erzählelemente/-fragmente, die ich ansetze, um eine narrative Struktur erfassen und reflektieren zu können: (1) eröffnende Erzählelemente, (2) ereignisanzeigende Erzählelemente, (3) abschließende Erzählelemente. Unter den drei genannten Kategorien erfasse ich im Folgenden textuelle und narrative Spezifika der Erzählelemente/-fragmente auf Twitter aus dem erstellten sekundären Subkorpus (elf diskontinuierliche Erzählungen mit insgesamt 132 Tweets) ${ }^{56}$.

\subsubsection{Merkmale und Funktionen eröffnender Erzählelemente}

Die Eröffnung diskontinuierlicher Erzählungen ist an der Leitfrage von Twitter orientiert, die lautet: Was gibt's Neues? Entsprechend beginnt die Eröffnung der Geschichte sehr oft mit Ankündigungen, die sprachlich auf die nahe Zukunft verweisen und für gewöhnlich wiederholt auftreten ${ }^{57}$. Das entspricht der Funktion

\footnotetext{
${ }^{56}$ Ich konzentriere mich auf spezifische Merkmale von Twitter unter narrativen Aspekten, um aufzuzeigen, wie die Internetplattform sich auf die Erzählungen auswirkt. Auf allgemeine sprachliche Merkmale in Tweets gehe ich nicht vertiefend ein. Diese sind bereits von der Linguistik sehr gut beforscht und beschrieben, siehe beispielsweise Moraldo (2012), Schlobinsky/Siever (2013).

${ }^{57}$ Einzige Ausnahme ist die Erzählung über die Wanderfalken „Fridolin und Frida“ von Telefonica Deutschland (Korpusquelle: Telef_782.2_TW). Die Erzählung wird aufgrund ihres Themas nicht vorab angekündigt.
} 
der Plattform, möglichst aktuell zu informieren. Nach diesem Musters eröffnen Volkswagen, Telefonica Deutschland, E.ON und die Deutsche Telekom ihre diskontinuierlichen Erzählungen. Als Erzählanlässe werden spezielle Veranstaltungen der Unternehmen benannt (Themenbereich: Aktuelle Informationen und Öffentlichkeitsarbeit). Mit einem Hashtag versehen werden sie zu einem festen Bestandteil der darauf folgenden Tweets (\#HM15, \#TEFberlin, \#tnt16). Die temporale Ankündigung ist hervorgehoben:

Teilnahme von Volkswagen an der Hannover Messe 2015 (Korpusquelle: VW_442_TW)

1. Tweet: „Ab morgen auf der @ @hannover_messe: \#VW Mensch-RoboterKooperation in der Fertigung. \#HM15“ (Volkswagen, Twitter, 2015-04-12)

2. Tweet: „Morgen Eröffnungsrundgang @ @hannover_messe: Kanzlerin \#Merkel und indischer Ministerpräsident @ narendramodi am \#VW Stand erwartet. \#HM 15“ (Volkswagen, Twitter, 2015-04-12)

3. Tweet: „Um 9 Uhr startet die @ hannover_messe 2015: \#VW präsentiert sich in Halle 15/Stand A04. \#HM15“ (Volkswagen, Twitter, 2015-04-12)

Eröffnung einer neuen Telefonica Deutschland Filiale in Berlin (2015) (Korpusquelle: Telef_787_TW)

1. Tweet: „Heute gibt's etwas ganz Besonderes in Berlin. Das hat die Hauptstadt noch nicht gesehen: \#TEFberlin ab 19 Uhr. (MG)“ (Telefonica Deutschland, Twitter, 2015-10-14)

2. Tweet: @ telefonica_de eröffnet heute ihren neuen Sitz in \#Berlin. Die Botschaft ist dabei.“ (Telefonica Deutschland, Twitter, 2015-10-14)

Beginn eines Telefonica Fan Events (Korpusquelle: Telef_786_TW)

1. Tweet: „O2 entdecken @o2entdecken: Wir freuen uns auf Euch: Heute um 17h fällt der Startschuss zum ersten \#o2FanEvent in der @o2WorldHamburg. \#Vorfreude“ (Telefonica Deutschland O2, Twitter, 2014-12-17)

Durchführung des Festival of Lights (Korpusquelle: E.ON_678_TW)

1. Tweet: „Die letzten Vorbereitungen laufen, gleich beginnt in \#Berlin das Festival of lights! \#lightsbyEON“ (E.ON, Twitter, 2015-10-09) 
Telekom Netz Tour 2016 (Korpusquelle: DTKOM_847.2..._TW_FB)

1. Tweet: „Countdown „Telekom Netz Tour 2016“ (tnt16) läuft: Ein erster BlogBeitrag [Internetadresse des Telekom Blog]“ (Jodl, Twitter, 2016-07-16)

2. Tweet: „\#Telekom Netz Tour 2016 \#tnt16 mit \#ConnectedBikes u. \#Wearables durch Baden-Württemberg [Linkadresse] (Deutsche Telekom, Twitter, 201607-19)

3. Tweet: „Ja, auch ich bereite mich auf die Telekom Netz Tour 2016 vor @ jodl @ telekomnetz@1Crossmax \#tnt16“ (stgoldmann, Twitter, 2016-07-19)

Page (2012) vergleicht in einer kontrastiven Studie das Vorkommen von Hashtags in Tweets von Privatpersonen, Prominenten und Unternehmen. Sie stellt die Frage, ob Hashtags konversationell sind und eine partizipatorische Kultur begünstigen. Ausgehend von einem Korpus mit 90.392 Tweets resümiert sie, dass Unternehmen Hashtags vornehmlich für Updates rund um ihre Belange nutzen, jedoch nur in geringem Maße für einen konversationellen Austausch. Inhaltlich sind die untersuchten Tweets von Unternehmen fast ausschließlich thematisch motiviert (Page 2012: 187 f.). Das kann ich für die von mir erhobenen Daten bestätigen. Vornehmlich zielten die Unternehmen in den von Page untersuchten Tweets darauf ab, sich selbst, ihr Motto und ihre Produkte auf diesem Wege sichtbar zu machen und sich nach Außen darzustellen (Page 2012: 198). Im Zusammenhang mit diskontinuierlichen Erzählungen sehe ich Hashtags in Unternehmens-Tweets als Mittel zur Kontextualisierung für das weitere narrative Gefüge, wie das folgende Beispiel eines eröffnenden Tweets von VW illustriert. Der Tweet stammt aus einer diskontinuierlichen Erzählung über die Hannover Messe 2015. Der Hashtag \#HM15 wurde in der Veranstaltungsankündigung etabliert und ist im Kreis der Rezipient/innen und/oder Follower/innen als bekannt anzunehmen. Entsprechend markiert er nachfolgende Zitate thematisch als zusammengehörig. Da in diskontinuierlichen Erzählungen die einzelnen Elemente nicht immer unbedingt direkt aufeinander folgen müssen, kontextualisiert der Hashtag das Zitat im Rahmen der Veranstaltung (Abb. 9.22): 


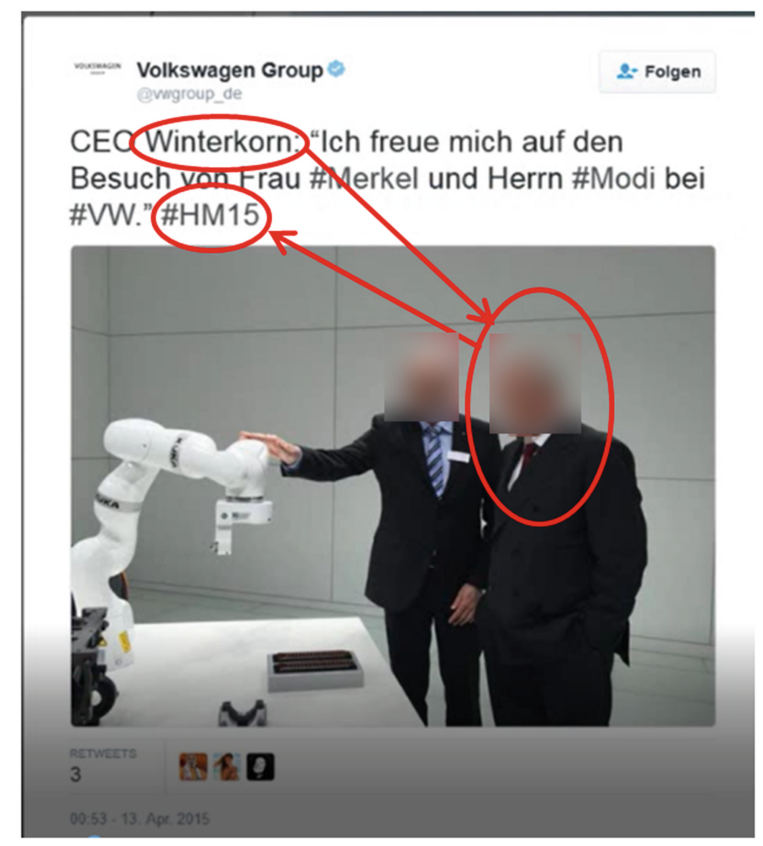

Abb.9.22 Hashtag zur Kontextualisierung eines Zitats (Volkswagen, Twitter, 2015-04-13. Korpusquelle: VW_442_TW)

Diesen Tweet in den Gesamtzusammenhang der diskontinuierlichen Erzählung einzuordnen, bedeutet für die Leser/innen, dass sie das Zitat und das Bild in mehreren Schritten mithilfe ihres Weltwissens (Wie sieht Herr Winterkorn aus? Was ist sein Betätigungsfeld?) verbinden müssen. Da der Hashtag auf die Hannover Messe referiert, kontextualisiert er einerseits textuelle Elemente innerhalb des Tweets (Herr Winterkorn spricht auf der Hannover Messe) und stiftet andererseits Kohärenz zwischen den Erzählfragmenten der entsprechenden diskontinuierlichen Erzählung (dieser Tweet gehört zu den Erzählfragmenten über die Hannover Messe).

Unter einer narrativen Perspektive haben die zuvor aufgezählten Ankündigungsbeispiele über die Eröffnung hinaus eine Funktion für die Gesamterzählung: Die Zukunftsverweise mittels der Temporaladverbien heute, morgen, gleich und konkreter Zeitangaben sowie ausgewählte präsentische Verben und Verbalphrasen wie starten, eröffnen, sich vorbereiten, letzte Vorbereitungen laufen und 
der Countdown läuft indizieren ein aktives Geschehen, dem eine gewisse Spannung innewohnt. Das Zusammenspiel der sprachlichen Merkmale in Verbindung mit der Wiederholung der Nachricht führt zu einem Spannungsaufbau. Hinzu kommt, dass ankündigende Tweets den Erzählanlass inhaltlich und emotional relevant setzen. Auf der inhaltlichen Ebene geschieht das durch Verweise auf innovative Produktvorführungen wie Mensch-Roboter-Kooperation, Wearables (tragbare Computer, die in die Sportbekleidung eingearbeitet sein können) oder Prominente, die den Anlass mit ihrem Besuch aufwerten sollen. Die emotionale Ansprache und die damit verbundene Relevanz unterstreichen die Autor/innen in Form ihrer eigenen Vorfreude auf das Ereignis (Wir freuen uns auf Euch und \#Vorfreude (Telefonica Dtl., Twitter, 2014-12-17. Korpusquelle: Telef_786_TW)) oder indem sie Interesse und Neugierde wecken durch Aussagen wie Heute gibt's etwas ganz Besonderes in Berlin. Das hat die Hauptstadt noch nicht gesehen (Telefonica Dtl., Twitter, 2015-10-14. Korpusquelle: Telef_787_TW). Bestandteile der Eröffnung sind hierbei die Bekanntgabe von Ort und Zeit, die Vorstellung von Akteuren, die Etablierung und damit einhergehend die Relevantsetzung des Themas.

Die Vorankündigung führt zu einem Spannungsaufbau, der zusätzlich von der Aktualität der Ereignisse getragen wird. Die zeitliche Nähe zu den Ereignissen kann als Strategie zur Involvierung der Rezipient/innen verstanden werden, da die Rezipient/innen direkt am Geschehen teilhaben. Finden sich keine vorankündigenden Tweets, beginnen auch in diesen Fällen die eröffnenden Elemente im Sinne der Vorgabe von Twitter mit sprachlichen Hinweisen auf Unmittelbarkeit: Jetzt geht's los, wir packen an (EnBW, Twitter, 29.01.2016. Korpuquelle: EnBW_720.3_TW_FB), Los geht's (Rewe, Twitter, 2015-10-03. Korpusquelle: REWE_386_TW), Auf geht's (Telekom, Twitter, 27-07-2016. Korpusquelle: DTKOM_847.2..._TW_FB).

\subsubsection{Merkmale ereigniskonstituierender Erzählelemente}

Inhaltlich ist die Ereignisdarstellung diskontinuierlicher Erzählungen für gewöhnlich von geringer Komplexität. In dem vorliegenden Korpus besteht die Ereignisdarstellung - mit Ausnahme der Netz Touren der Deutschen Telekom - aus einem Ereignis, das die Autor/innen fraktioniert twittern. Dabei handelt es sich ausnahmslos um Ereignisse, die die Unternehmen als fachliche kompetent und/oder verantwortungsbewusst zeigen. Charakteristisch ist außerdem, dass diskontinuierliche Erzählungen äußerst selten eine Komplikation und deren Auflösung beinhalten. Sollte das doch der Fall sein, dann handelt es sich um unerwartete Einflüsse (vergleichbar mit bimodalen Kompakterzählungen auf Facebook), 
die lediglich den Ablauf der geplanten Vorgänge verändern: z. B. nicht funktionierendes technisches Equipment (Telekom Netz Tour 2016). In der Regel besteht das Erzählenswerte in einem sozial relevanten außergewöhnlichen Ereignis (der Besuch der Bundeskanzlerin am Messestand von VW) und/oder dem damit einhergehenden Erlebnis für alle Beteiligten (Erfahrungshaftigkeit, siehe Abschn. 5.2.2). Direkte sprachliche Verweise auf die Erzählwürdigkeit eines Ereignisses und des damit verbundenen Erlebnisses geschehen durch die Verwendung des Superlativs beste Stimmung oder in tautologischer Verwendung absolutes Highlight und durch die Verwendung von positiv konnotierten Substantiven ein Augenschmaus (E.ON, Festival of lights in Berlin 2015, Twitter), Phrasen Drama aufdem Feldberg oder Adjektiven [g]eheime Einblicke, grandioser Ausblick, wunderschönes Münster (Telekom, Netz Tour 2016, Twitter).

Zusätzlich kann die Ereignisdarstellung durch die Wiedergabe von Redebeiträgen von geschichtsinternen Akteuren gestaltet werden. Insofern die Redewiedergabe schriftlich erfolgt ${ }^{58}$, kann sie in direkte und indirekte Rede unterschieden werden $^{59}$. Neben Volkswagen wendet Telefonica Deutschland diese Option an. Auffällig ist dabei die hohe Frequenz direkter Redewiedergaben als ein Mittel, um die Ereignisse in Tweets zu reproduzieren. Orthografische Konventionen zur Markierung der Redewiedergabe spielen in diesen Fällen eine untergeordnete Rolle. Kenntlich gemacht werden sie in verschiedenen orthografischen Varianten zusammen mit verba dicendi, welche voran- oder nachgestellt werden. Dabei erhöht die Anzahl von zitierten bekannten Persönlichkeiten die Bedeutung des Events und damit des Unternehmens. Aus der narrativen Perspektive führt die dichte Abfolge der Zitate dazu, dass die erzählte Zeit gerafft, also beschleunigt wird. Das impliziert Kurzweile und Dynamik.

Ebenfalls dynamisierend auf die Ereignisdarstellung wirken Tweets, die sich in das Hier-und-Jetzt verorten, indem der/die Erzähler/in den Eindruck erweckt, ihre Origo wäre deckungsgleich mit dem Erzählten. Georgakopoulou nennt solche Geschichten von privaten Nutzer/innen breaking news (ebd. 2015: 260). Kennzeichnend dafür ist, dass die Verfasser/innen auf aktuelle und sich gerade

\footnotetext{
${ }^{58}$ Also nicht im Bewegtbild wie in dem Beispiel über den EnBW-Macher Bus und die Mountainbikestrecke in St. Märgen

59 Thüne (2008) verweist für gesprächsanlytische Untersuchungen darauf, dass die Dichotomie von direkter und indirekter Redewiedergabe unzulänglich ist. Bereits Brünner (1991) habe ,auf die enorme Vielzahl von möglichen Formen der Redewiedergabe hingewiesen“ und Günthner (2000) habe ,verschiedene Zwischenformen herausgefiltert und gezeigt, dass es in gesprochener Sprache wenig sinnvoll ist, von zwei voneinander getrennten Idealformen von Redewiedergabe auszugehen, sondern dass vielmehr ein Kontinuum existiert, das von maximaler Direktheit zu maximaler Indirektheit reicht" (Thüne 2008: 4).
} 
entwickelnde Ereignisse referieren. Die Geschichte wird sukzessive im Prozess aktualisiert, sodass der Eindruck eines ,speaking and doing“ (Puschmann/Heyd 2012: 175) entsteht. Nach Georgakopoulou (2015) geschieht dies aus dem Bedürfnis heraus, sich möglichst dynamisch und kontinuierlich mit Freunden auszutauschen. Zwar handelt es sich bei den Rezipient/innen diskontinuierlicher Erzählungen nicht um Freunde, wohl aber in den meisten Fällen um Follower/inen, die ebenso auf den neuesten Stand der Entwicklung gebracht werden wollen. Entsprechend knüpfen Unternehmen an die bereits bestehende Konvention aus dem privaten Anwendungskontext an. Page (2013) analysiert das Phänomen auf Basis eines Korpus (51.643 Tweets) von Tweets von Prominenten. Mit diesen Mitteln - so Page - werde ein anhaltendes Gefühl der Präsenz zwischen den Verfasser/innen und dem Publikum aufrechterhalten. Sie verweist auf Bennett (2000), der die Wirkung geteilter Zeit auf die zwischenmenschliche Beziehung untersucht und zu dem Ergebnis kommt, dass so die Illusion von Realitätsnähe entstehe. (Page 2013: 103) Der Unterschied zwischen diskontinuierlichen Erzählungen von Unternehmen und breaking news von Privatpersonen liegt letztlich darin, dass Tweets von Unternehmen einen höheren Planungsgrad hinsichtlich der Inhalte und der Veröffentlichung haben (strategische Kommunikation) als private Tweets. Daraus resultiert, dass Tweets aus dem Unternehmenskontext eben nicht immer aus dem implizierten „Hier und Jetzt“ heraus entstehen. Das wird beispielsweise an dem Temporaladverb gestern deutlich. Um dennoch zeitliche Nähe zu suggerieren, können die Autor/innen weitere Vergangenheitsmarker tilgen. In dem folgenden Beispiel tilgt der/die Autor/in die Vergangenheitsform des Vollverbs ,sein“ und produziert eine Struktur-Ellipse (Zifonun et al. 1997: 435)

„Beste Stimmung [war] gestern in \#Berlin bei der Festival of Lights-Eröffnung. Um 19 Uhr geht's heute weiter“ \#lightsbyEON“" (E.ON, Twitter, 2015-10-10. Korpusquelle: E.ON_678_TW)

Zusätzlich wendet der/die Verfasser/in im Folgesatz den Blick in die Zukunft und schlägt so eine Brücke zwischen gestern und heute, indem er/sie in Aussicht stellt, dass die beste Stimmung kontinuierlich fortbesteht.

Eine komplexe, ausgebaute Ereignisdarstellung setzen die Autor/innen der Deutschen Telekom in der uRE über den LTE-Ausbau aufder Zugspitze 2015 (Korpusquelle: DTKOM_809.1-13_TW) und über die wiederkehrende Aktion Telekom Netz Tour um. Im Folgenden behandle ich die textuelle Komplexität, die sich auf die Twittersyntax bezieht. Exemplarisch verdeutliche ich sie anhand von Auszügen der Geschichte über die Telekom Netz Tour 2016. Die Telekom Netz Tour 
2016 ist ein Projekt der Deutschen Telekom, einmal im Jahr reist ein Team des Unternehmens durch einen Bereich des Landes. 2016 ging die Reise durch den Südwesten Deutschlands. Einer der Protagonisten (PR3) begleitet die Tour als Moderator und übernimmt in weiten Teilen die Aufbereitung der Erzählung. Zwei weitere Tourteilnehmer (Protagonist 1 (PR1) und Protagonist 2 (PR2)) bewältigen die Tour auf Connected Bikes bekleidet mit Wearables. Das Ziel der Aktion ist es, zu zeigen, wie es um die Digitalisierung im Land bestellt ist. Das Ende der Tour krönt ein Radrennen der beiden Radfahrer auf den Feldberg zwischen den beiden Radfahrern. Die „Abenteuer“ und Erlebnisse der drei Protagonisten bilden die Geschichte. Die Twittersyntax der uRE ist komplex, Erzähler/innen und Adressat/innenen wechseln, was das @-Präfix markiert. Das @-Präfix kann an unterschiedlichen Stellen im Tweet stehen. In der „Kopfzeile“ markiert es den Nutzernamen (Unterstreichung):

\section{Protagonist zwei @PR2 23. Juli 2016 \\ a.PR3@PR1: Hole mir für \#tnt16 Tipps vom Bürgermeister aus Ühlingen-Birkenfeld, erlebe Schwarzwald hautnah.}

Steht das@-Zeichen mit einem Nutzerinnennamen am Beginn eines Tweets, handelt es sich um eine Antwort auf einen vorausgegangenen Tweet (blaue Unterstreichung):

\section{Protagonist eins@PR125. Juli 2016}

Der müde Krieger@PR2 \#tnt16 angekommen in Badenweiler.

Und steht das@-Zeichen letztlich an einer anderen Stelle innerhalb des Tweets, dann behandelt Twitter das als eine „Erwähnung“ (blaue Unterstreichung):

Hauptfigur eins@PR1 25. Juli 2016

Der müde Krieger @PR2 \#tnt16 angekommen in Badenweiler.

Bezieht sich das @-Präfix auf eine Antwort oder eine Erwähnung, erscheint der Tweet auf der Twitter-Startseite der betroffenen Person, damit sie ggf. darauf reagieren kann. Ist das innerhalb einer diskontinuierlichen Geschichte gegeben, folgt daraus, dass sich die verschiedenen Erzähler/innen aufeinander beziehen, indem sie sich gegenseitig adressieren. Auf diese Weise kommt es zu einer zeitlich zerdehnten Aktion - Reaktion zwischen den Protagonist/innen der Gesamterzählung, was die Rezipient/innen anhand der @-Markierung erkennen. Daraus ergibt sich eine komplexe narrative Vermittlung, denn es liegt keine Erzählerrede vor, die die Rezipient/innen durch die Geschichte führt. Es handelt sich in diesem Fall um Erzähler/innen in der zweiten Instanz (entspr. der zweiten Grundvoraussetzung im 
Verortungsschema, Abschn. 5.4). Durch die Kommunikation der drei Protagonisten $^{60}$ miteinander wechseln die „Erzähler/innen“, sodass die Geschichte aus dem Bezug der einzelnen Tweets herausgelesen werden muss. Zur Veranschaulichung folgt eine Sequenz vom Beginn der Netz Tour 2016, in der die Protagonisten miteinander und gleichzeitig öffentlich auf Twitter kommunizieren. Die Twitterer (= Protagonisten, PR1 / PR2 / PR3) sind fett markiert, um die Bezüge aufeinander zu verdeutlichen. Die Tweets sind in der ersten Spalte durchnummeriert. In der zweiten Spalte stehen die verfassenden Protagonisten und das Veröffentlichungsdatum. In der dritten Spalte findet sich der dazugehörige Tweet. Die Interaktion erkläre ich unter der Tabelle:

(Bsp.) Erzähltextsequenz: Erzähler zweiter Instanz am Beginn der diskontinuierlichen Erzählung über die Telekom Netz, Tour 2016. Korpusquelle: DTKOM_847.2..._TW_FB.

\begin{tabular}{l|l|l}
\hline \multirow{2}{*}{ Nr. } & Thema: Vorbereitungen für den ersten Tour Tag \\
\cline { 2 - 3 } & $\begin{array}{l}\text { Protagonisten: } \\
\text { Verfasser, Veröffentlichungsdatum }\end{array}$ & Twittertext \\
\hline $\mathbf{5}$ & $\begin{array}{l}\text { Protagonist 3: } \\
\text { @ telekomnetz, 22. Juli 2016 }\end{array}$ & $\begin{array}{l}\text { „Morgen treffe ich mich mit @ PR1 und @ PR2 in } \\
\text { Emmendingen zum \#tnt16 Start. Was darf ich } \\
\text { nicht vergessen?“ [zusätzlich ist ein Selbstportrait } \\
\text { mit einer Steckdosenleiste abgebildet }]\end{array}$ \\
\hline $\mathbf{6}$ & $\begin{array}{l}\text { Protagonist 1: } \\
\text { @PR1, 23. Juli 2016 }\end{array}$ & $\begin{array}{l}\text { „Meine Produktionseinheit ... Wieviel Platz ist im } \\
\text { Ford Focus, sagtest du @ PR3? ,tnt16 } \\
\text { @ telekomnetz“ [zusätzlich ist ein Foto mit } \\
\text { technischen Utensilien abgebildet }]\end{array}$ \\
\hline $\mathbf{7}$ & $\begin{array}{l}\text { Protagonist 2: } \\
\text { @PR2, 23. Juli 2016 }\end{array}$ & $\begin{array}{l}\text { „PR3 @ PR1: Hole mir für \#tnt16 Tipps vom } \\
\text { Bürgermeister aus Ühlingen-Birkenfeld, erlebe } \\
\text { Schwarzwald hautnah.“ [zusätzlich sind zwei } \\
\text { Fotos von sich mit dem Bürgermeister und einer } \\
\text { Karte, die sie gemeinsam betrachten, abgebildet }]\end{array}$ \\
\hline $\mathbf{8}$ & $\begin{array}{l}\text { Protagonist 1: } \\
\text { @PR1, 23. Juli 2016 }\end{array}$ & $\begin{array}{l}\text { @PR3 Komprimiert. Zufrieden?“ [zusätzlich ist } \\
\text { ein Foto mit einem befïlten Rucksack abgebildet }]\end{array}$ \\
\hline
\end{tabular}

PR3 (Tweet 5) adressiert seine zwei Kollegen mit der Information, dass er gerade letzte Vorbereitungen für die Tour erledigt und auch die Steckdosenleiste nicht vergessen hat. Darauf meldet sich PR1 (Tweet 6) mit einem Foto weiterer technischer Utensilien und der scherzhaft gemeinten Frage an PR3: Wieviel Platz ist im Ford Focus, sagtest du @ PR3? An das Thema, Vorbereitung ' schießt sich in Tweet 7 PR2 an und benennt, welcher vorbereitenden Tätigkeit er noch nachgeht

${ }^{60}$ Es handelt sich um drei männliche Personen, wie später folgende Fotografien belegen, daher gendere ich in diesem Kontext nicht. 
(Tipps einholen). PR1 schließt die Interaktion damit ab (Tweet 8), dass er noch einmal scherzhaft auf den zuvor bei PR3 angefragten Stauraum eingeht. Er twittert infolgedessen das Foto eines gepackten Rucksacks und stellt die rhetorische Frage, ob er seine Utensilien ausreichend komprimiert habe und sein Kollege (PR3) zufrieden damit sei. Das Beispiel zeigt, wie anhand der Twitterkonventionen für Texte die Figurenrede innerhalb der Erzählung organisiert wird und wie sie sich aufeinander beziehen. Die Rezipient/innen lernen infolge der Interaktion die Akteure der Gesamterzählung kennen, werden in die Vorbereitungen einbezogen und bekommen so die vorfreudige Aufbruchstimmung vermittelt.

Die Ereignisdarstellung stützt sich in den Beispielen auf einen nach außen getragenen Informationsaustausch. Der Austausch kann die Rezipient/innen inkludieren und exkludieren. Solange die Protagonist/innen Personen adressieren, die den Leser/innen bekannt sind, sind sie inkludiert. Findet der Informationsaustausch jedoch mit unbekannten Personen statt, sind die Rezipient/innen exkludiert. Der folgende Tweet veranschaulicht eine Exklusion. In dem folgenden Beispiel macht PR3 einen „Insiderwitz“, den er an eine den Leser/innen unbekannte Person ${ }^{61}$ adressiert (umrandet) (Abb. 9.23):

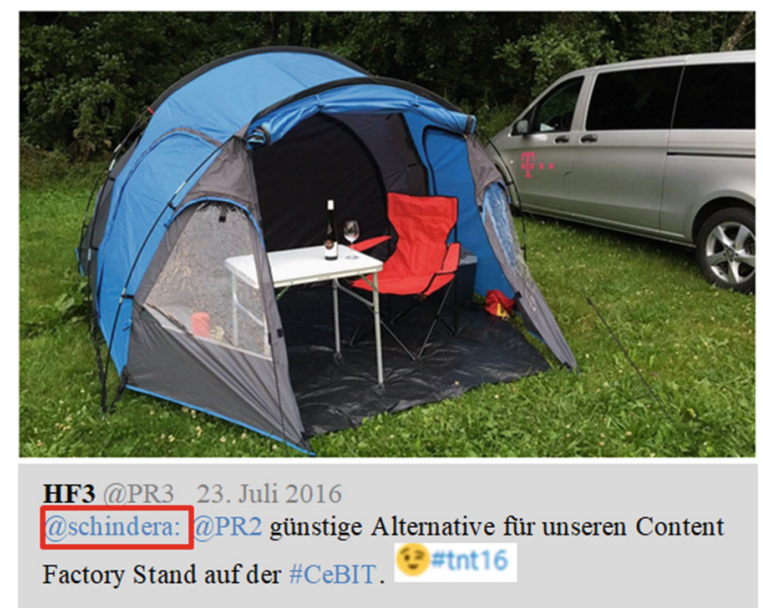

Abb.9.23 Exklusion der Rezipient/innen: „Insiderwitz“ (Jodl, Twitter, 2016-07-23. Korpusquelle: DTKOM_847.2.2_TW)

${ }^{61}$ Es handelt sich um seinen Vorgesetzten aus der Unternehmenskommunikation. 
Einzig die Erwähnung von PR2 (@PR2) und der Hashtag \#tnt16 stellen eine Verbindung zu der Gesamterzählung her. Die Aussage günstige Alternative für unseren Content Facatory Stand auf der \#CeBIT animiert unser Kohärenzbedürfnis dahingehend, die eingebundene Fotografie in den Gesamtzusammenhang der Geschichte zu stellen. PR3 kontrastiert das Foto eines Zelts mit einem Messestand auf der CeBIT. So entsteht ein Insiderwitz, den er aber offensichtlich in der zweiten Funktion (Information) als einen Bestandteil der Gesamterzählung um die Telekom Netz Tour 2016 einbringt - die Protagonisten haben wohl auf ihrer Tour auch in einem Zelt übernachtet.

Die Komplexität ereignisanzeigender Erzählelemente entsteht also dadurch, dass kein Erzähler die Leser/innen führt (Erzähler in der ersten Instanz), sondern sie sich vielmehr mithilfe ihres Wissens über die Twittersyntax und anhand der Interaktion zwischen den Protagonisten die Geschichte ,zusammenreimen“ müssen. Der Vorgang erschwert sich zusätzlich, wenn die Protagonisten auf „Insiderwissen“ anspielen und/oder Unbekannte adressieren.

\subsubsection{Abschließende Erzählelemente}

Ausgehend von verschiedenen narrativen Modellen schließt ein/e Erzähler/in für gewöhnlich ihre Geschichte ab und bindet sie ggf. in das Hier und Jetzt zurück. In bimodalen Kompakterzählungen auf Facebook sind es insbesondere Bewertungen, die diese Funktion erfüllen sowie Verlinkungen auf weiterführende Informationen. In diskontinuierlichen Erzählungen auf Twitter stellt sich die Situation anders dar: sowohl Erzählungen von Privatpersonen als auch uER von Unternehmen bleiben auf Twitter häufig offen. Hintergrund dafür ist der Anspruch, aus einem aktuellen Geschehen heraus direkt zu erzählen. Eine besonders ausgearbeitete, homogene oder eben auch geschlossene Gesamterzählung zu produzieren, ist zweitrangig. In den elf untersuchten diskontinuierlichen Erzählungen verfügen fünf von 17 über ein Element, das als Endpunkt gewertet werden kann. Die Schlussgestaltung ist dabei verschieden. Eine Möglichkeit, den Schluss zu gestalten, ist eine zweckgebundene Ergebnisbenennung - wie sie beispielsweise am Ende der Telekom Netz Tour 2016 erscheint (siehe das Beispiel unten). In diesem Fall tritt der/die Erzähler/in ,aus der vergangenen Welt der erzählten Geschichte heraus“" (Brinker et al. 2014: 68) und deutet das Vergangene auf einer übergeordneten Reflexionsebene:

„Entlang am Rhein und unterwegs im Schwarzwald - die Telekom hat im Sommer gezeigt, was der Netzausbau im Süden von Baden-Württemberg erreicht hat, welche Technik er verwendet und wie er das Leben der Menschen vor Ort verändert hat. Ein Rückblick." (Deutsche Telekom, Twitter, 2016-10-16. Korpusquelle: DTKOM_847.2.2_TW) 
In diesem Beispiel benennt PR3 rückblickend die Funktion der Veranstaltung, was einer zweckgebundenen Ergebnisbenennung entspricht. Dass diese Einschätzung nicht immer von einem/r Mitarbeiter/in des Unternehmens kommen muss, zeigt uns Telefonica Dtl. am Ende der Erzählung über das O2-FanEvent 2014. Das Unternehmen postet selbst verschiedene Fotografien unter dem Titel Impressionen vom \#o2FanEvent und beendet seine Twitteraktivitäten zu der Veranstaltung mit der Veröffentlichung des Retweets eines Gastes. Die Markierung eines Retweets mit dem Kürzel ,RT' ist nicht verpflichtend. Ich habe es in diesem Beispiel mit eckigen Klammern zur Verdeutlichung eingefügt:

„[RT] Congrats to @O2entdecken, tolles erstes Fan Event! Es war mir eine Ehre“ [Link zu der Instagram-Seite des Gastes] (Privatperson, auf der Twitterseite von Telefonica Deutschland, 2014-12-17. Korpusquelle: Telef_786_TW)

Schlussgestaltungen, die eher der gängigen Tweetgestaltung gleichen, zeichnen sich dadurch aus, dass die Erzählung inhaltlich-thematisch als geschlossen betrachtet werden kann. In dem Fall des EnBW-Macher-Busses in St. Märgen ist das Projekt abgeschlossen. Die dabei entstandene Mountainbikestrecke wird im letzten Tweet von den Anwesenden genutzt (Bewegtbild) und bewertet:

„\#EnBW \#MacherBus in St. Märgen: die neue Mountainbike-Strecke hält was Sie verspricht! \#WirMachenDasSchon" (EnBW, Twitter, 2016-06-30. Korpusquelle: EnBW_719.4_TW_FB) [angefügtes Bewegtbild von Betroffenen, die auf der neuen Strecke sowohl fahren wie auch sitzen und sich gemeinsam darüber freuen.]

Eine Schlussgestaltung anhand lexikalischer Marker, die im Weltwissen der Rezipient/innen als ein Ende markierend gespeichert ist, zeigt das nächste Beispiel von Telefonica Dtl::

„Jetzt läuft die super Aftershow-Party bei \#TEFberlin (MG)“ (Telefonica Deutschland, Twitter, 2015-10-14. Korpusquelle: Telef_787_TW)

In dem Tweet indiziert das Kompositum Aftershow-Party, die bekanntlich am Ende einer Veranstaltung stattfindet, den Schluss der Gesamtveranstaltung.

Neben solchen inhaltlich motivierten Abschlüssen diskontinuierlicher Erzählungen findet sich eine weitere Option der Schlussgestaltung. Sie ist für den Kontext der sozialen Medien charakteristisch und tritt auch auf anderen Plattformen auf. Dabei handelt es sich um den Wechsel des Satzmodus durch expressive Sprechakte des Dankens, Lobens und Beglückwünschens - hierzu folgendes Beispiel von Rewe, das am Ende der diskontinuierlichen Erzählung über die Unterstützung von Rewe beim Kölnmarathon steht: 
„Glückwunsch an alle Läufer/innen des \#koelnmarathon! Gleich welche Strecke u. Zeit, wer ankommt ist immer ein Sieger!“ (Rewe, Twitter, 2015-10-04. Korpusquelle: REWE_386_TW)

Dass diskontinuierliche Erzählungen über eine Schlussmarkierung verfügen, ist nicht unbedingt erforderlich. Die Konvention, in Tweets die Gegenwart zu reflektieren bzw. möglichst nahe am Geschehen zu berichten, führt dazu, dass diskontinuierliche Erzählungen, ohne Irritationen auszulösen, offen bleiben können: Ist das Wichtigste gesagt, wird die Erzählung eingestellt. Hier liegt das strategische Kalkül der Unternehmenskommunikation, denn keine der Erzählungen endet, bevor nicht etwas für die (S)D Relevantes getwittert wurde. Der Abbruch findet somit erst nach dem besonderen Ereignis statt, wie die folgenden Beispiele zeigen (fett markiert):

(Bsp. 1) „Er war das Highlight des heutigen Abends: @robbiewilliams live [...]“ (Volkswagen, Twitter, 2015-04-28. Korpusquelle: VW_455_TW)

(Bsp. 2) „...und last but not least das absolute Highlight des Festivals of Lights: das [illuminierte] Brandenburger Tor!“ (E.ON, Twitter, 2015-10-10. Korpusquelle: E.ON_678_TW)

(Bsp. 3) „Die Schneebälle wachsen! Unglaublich, wie groß unsere Wanderfalken nach nur 3 Wochen sind“" (Telefonica Deutschland, Twitter, 2015-04-17. Korpusquelle: Telef_782.2_TW)

Während in den zwei ersten Beispielen der Anglizismus Highlight explizit auf selbstdarstellungsrelevante Merkmale verweist (Auftritt eines Prominenten, Beitrag von E.ON zu einer kunstvollen Illumination), geschieht dies im dritten Beispiel implizit durch den Hinweis darauf, dass die Wanderfalkenküken auf besagtem Telefonmast unter der Obhut des Unternehmens wachsen und gedeihen.

Wie sich bereits in den bislang zitierten Beispiele andeutet, sind diskontinuierliche Erzählungen vermehrt mit Bildern und insbesondere Bewegtbildern angereichert. Hierbei werden auch ,umfangreichere“ Filme eingebunden. Das unterscheidet diskontinuierliche Erzählungen von bimodalen Kompakterzählungen auf Facebook. 


\subsubsection{Multimodale Aspekte in diskontinuierlichen Erzählungen auf Twitter}

Die erhobenen diskontinuierlichen Erzählungen funktionieren als Zusammenspiel von wenig Schrifttext mit reichlich Bildern und Bewegtbildern. Die Ursache dafür sind nicht die technische Möglichkeiten von Twitter, sie einbinden zu können. Dahinter stehen tiefgreifendere Beweggründe, auf die die Rezeptionsforschung schlüssige Antworten bietet. So untersuchten beispielsweise Godulla/Wolf (2015; 2017) multimodale Erzählungen im Internet im Rahmen einer kommunikationsund medienwissenschaftlich angelegten Studie über die Rezeption von Scrollytelling $^{62}$, Webdokumentationen ${ }^{63}$ und selektiven Multimediastorys ${ }^{64}$. Sie nennen diese Darstellungsformen: „Geschichten orientierte Langformen“ (Godulla/Wolf 2017: 62). Zwar handelt es sich bei diskontinuierlichen Erzählungen nicht um eine zusammenhängende Langform, doch teilen sie mit jenen Langformen die narrative Realisierung im Internet sowie die Einbindung multimodaler Elemente. Godulla/Wolf führten ,gemeinsam mit Studierenden der Universitäten Passau und Leipzig“" (Godulla/Wolf 2017: 67) eine Rezeptionsstudie im Rahmen eines Lehrforschungsprojekts durch. Die Autorin und der Autor belegen darin, was in der Literatur meist als Tatsache vorangenommen wird: So waren es insbesondere die visuellen Formate (Fotoslides, animierte Fotos, Videos), die die Proband/innen positiv bewerteten. In den Interviews gaben sie an, dass es das multimodale Zusammenspiel der verschiedenen Kommunikate (die Autor/innen verwenden den Begriff „Medienelemente“) war, das sie in die Geschichte hineinzog. Hinzukam, dass sich die meisten Befragten im Nachhinein besonders gut an Fotos und Videos erinnern konnten. Für rund 33 Prozent der Proband/innen erhöhte sich das spielerische Erleben durch die multimodalen Darstellungen und die damit verbundene Selektivität. Rund 44 Prozent der Interviewten konzentrierten sich ausschließlich auf Fotos und Videos, während sie den Text außer Acht ließen. (Godulla/Wolf 2017: 70 ff.) Daraus lässt sich für die Produzent/innen digitaler Erzählungen ableiten, dass Bilder und insbesondere Bewegtbilder die Aufmerksamkeit binden und multimodale Kombinationen dem Informationsvorgang ein unterhaltendes

\footnotetext{
${ }^{62}$ Das Portmanteauwort setzt sich aus den Wörtern „Storytelling“ und „Scrollen“ zusammen. Es bezieht sich auf Texte, die in linearer Abfolge ,durch scrollen von oben nach unten bzw. links nach rechts rezipiert [werden]“ (Godulla/Wolf 2017: 67).

${ }^{63}$ Eine Webdokumentation zeichnet sich durch den primären Einsatz von Videoelementen aus, deren Abfolge den Rezipient/innen obliegt. (ebd.)

${ }^{64}$ Eine selektive Multimediastory kennzeichnet, dass sie nur mithilfe eines über (Touch-) Gesten steuerbaren mobilen Endgeräts rezipiert werden kann. (ebd.)
} 
Moment beifügen. Das erhöht die Erinnerung an sowie die Offenheit für mögliche Botschaften - somit auch für die (S)D von Unternehmen. Föhl/Huck-Sandhu (2017) benennen einleitend zu ihrer Studie, die in den Public Relations und der Werbekommunikation verortet ist, relevante Forschungserkenntnisse zu der Rolle von multimodalen Darbietungsformen. Ihr Interesse wenden sie darauf, wie Rezipient/innen Inhalte aufnehmen und akzeptieren. Sie verweisen auf eine These aus der Konsumentenforschung, nach der eine Produktpräsentation umso attraktiver wahrgenommen wird, ,je mehr Multimediale-Elemente zum Einsatz kommen“ (Bayter/Ashdown 2014: 33, nach ebd. 2017: 209). Darüber hinaus führen die Autorin und der Autor ein Studienergebnis von Occa/Sugg (2016) an, dem zufolge narrativ aufbereitete Videos vor allem auf die Einstellung und Verhaltensabsichten der Rezipient/innen wirken. Da ich in dieser Arbeit imagefördernde diskontinuierliche Erzählungen untersuche, spielen sowohl die Attraktivität der Inhalte (als motivierendes Element, die Erzählung überhaupt weiter zu verfolgen) als auch der Wunsch, auf die Einstellung von Bezugsgruppen zum Unternehmen Einfluss zu nehmen, eine Rolle. Diese Überlegungen in Verbindung mit den zuvor benannten Aspekten über die Offenheit für und Erinnerung an die kommunizierte Botschaft können als Motivation dafür gesehen werden, diskontinuierliche Erzählungen multimodal zu präsentieren.

Um zu illustrieren, wie diskontinuierliche Erzählungen multimodal angelegt sind und welchen Beitrag multimodale Elemente für die Gesamterzählung leisten können, beziehe ich mich auf die in Ausschnitten zitierte Telekom Netz Tour 2016. Ich greife auf diese diskontinuierliche Erzählung als Beispiel zurück, da die Autoren darin sämtliche Möglichkeiten der multimodalen Gestaltung verwenden, die Twitter technisch anbietet. So kann ich multimodale Merkmale, die auch in anderen diskontinuierlichen Erzählungen (nur nicht in diesem Ausmaß) auftreten, miterfassen. Ausgehend von den einzelnen Bestandteilen (Tweets) der Erzählung treten in diskontinuierlichen Erzählungen folgende multimodale Realisierungen auf:

- Tweets mit Text und der Bildsorte ,Fotografie“

- Tweets mit Text und audiovisuellen Bewegtbildern (meist Filme per Handykamera)

- Tweets mit Text und seriell filmischen Erzählelementen (meist Filme von YouTube) 


\subsubsection{Spezifik und Integration von Fotografien}

In Tweets eingebundene Fotografien sind in diskontinuierlichen Erzählungen anders als in der bimodalen Kompakterzählung auf Facebook - nicht an das Ende der Erzählung gebunden, sondern sie sind innerhalb der Gesamterzählung angesiedelt. Das räumlich-syntaktische Muster (Stöckl 2010: 56) innerhalb eines Tweets, also die räumliche Position von Schrift und Bild, ist für gewöhnlich linearisiert. Das bedeutet, Text und Bild sind positionell voneinander abgegrenzt, indem die Schrift entweder ober- oder unterhalb der Fotografie platziert ist. Eine Ausnahme zeigt die folgende Abbildung (Abb. 9.24):

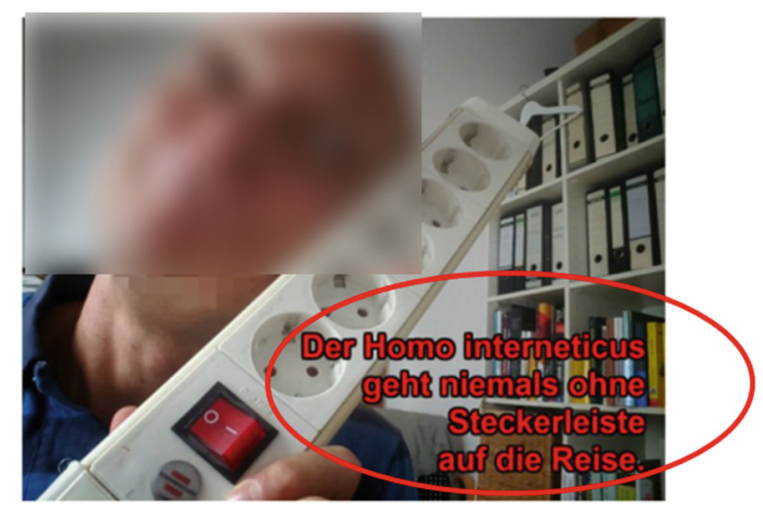

Abb.9.24 Simultanes Verknüpfungsmuster von Text und Bild (Deutsche Telekom, Twitter, 2016-07-22. Korpusquelle: DTKOM_847.2.2_TW)

Der Tweet stammt aus einer Sequenz über die Vorbereitungen zum ersten Tour-Tag der Telekom Netz Tour 2016. Es geht um das Organisieren, Packen u. a. In diesem Beispiel liegt ein simultanes Verknüpfungsmuster (Stöckl 2010: 57) vor. Es zeichnet sich dadurch aus, dass der Schriftzug in das Bild integriert ist (im Bild eingekreist). Dabei handelt es sich um eine kommentierende Erläuterung (konfiguriertes Muster, Stöckl 2010: 58), die uns durch die Nähe von Text und Bild impliziert: die Person neben der Steckleiste ist ein Homo interneticus ${ }^{65}$.

Beide Anordnungsmuster von Text und Bild (linearisiert und simultankonfiguriert) führen während des Rezeptionsvorgangs dazu, dass textuelle und bildliche Elemente ,allerdings in unterschiedlichem Maße ineinander integriert

${ }^{65}$ Ein humorvoller Neologismus für eine Person, die viel Zeit im und mit dem Internet verbringt. 
und zu einem Gesamttext synthetisiert werden“ (Stöckl 2004: 97). Von Interesse für die Untersuchung sind diesbezüglich spezifische Merkmale der Kopplung von Text und Bild: Es stellt sich die Frage, wie sich Text und Bild in dem Kontext aufeinander beziehen (syntaktisch-text-strukturelle Aspekte) und welchen Beitrag Fotografien in der Erzählung leisten (semantische Koppelung) (siehe Stöckl 2004: 141). Beide Fragen richten sich auf informationsbezogene Aspekte von Text-Bild-Beziehungen.

Ausgehend von einer logozentrischen Perspektive benötigen wir, um die Botschaft der Bilder im Rahmen der Erzählung entschlüsseln zu können, den sprachlichen Begleittext (vgl. Nöth 2000: 492): Da Bilder über einen Bedeutungsüberschuss verfügen (Stöckl 2010: 48), sind es sprachliche und inhaltliche Bezüge, die den Blick der Betrachter/innen auf den entsprechenden Bildausschnitt lenken.

Fotografien haben einen denotierenden Bezug zu der Wirklichkeit. Das bedeutet, sie beziehen sich auf Objekte in der realen Welt (Scholz 1991, 1998; nach Stöckl 2004: 115). Diese Objekte sind in den Fotografien der erhobenen diskontinuierlichen Erzählungen überwiegend Personen und Gegenstände. Ebenso wie in bimodalen Kompakterzählungen auf Facebook bilden Fotos in diskontinuierlichen Erzählungen in der Mehrzahl Personen ab (ca. 76 Prozent aller Fotografien zeigen Personen ${ }^{66}$ ). Das vermehrte Vorkommen von Personen auf Fotografien lässt sich dadurch erklären, dass Personen als Handlungsträger/innen und Erlebende grundlegende Bestandteile einer Erzählung sind. Außerdem entsteht dadurch eine Personalisierung der Akteure, die Nähe herstellt und das Interesse der Rezipient/innen an der Erzählung erhöht.

Der sprachliche Bezug auf Fotografien mit Personen (= syntaktisch-textstrukturelle Aspekte) erfolgt auf vier verschiedene Arten. (1) Der naheliegendste Bezug ist pronominaler Art. In dem folgenden Beispiel ist eine Person auf dem Foto zu sehen (PR2), entsprechend referiert der Text in der 1. Person Singular auf diese Person (Abb. 9.25):

\footnotetext{
${ }^{66}$ Insgesamt liegen 91 Fotos in den erhobenen diskontinuierlichen Erzählungen vor. Davon bilden 69 Fotos vor allem Personen ab. Nicht dazu gezählt wurden Bewegtbilder. Sie werden separat im folgenden Kapitel behandelt. In Bewegtbildern kommen vor allem noch räumliche Aspekte hinzu.
} 
Abb.9.25 Sprachlicher Bezug auf Personen in der 1. P.S. auf das Foto (PR2, Twitter, 2016-07-20.

Korpusquelle: DTKOM_847.2.2_TW)

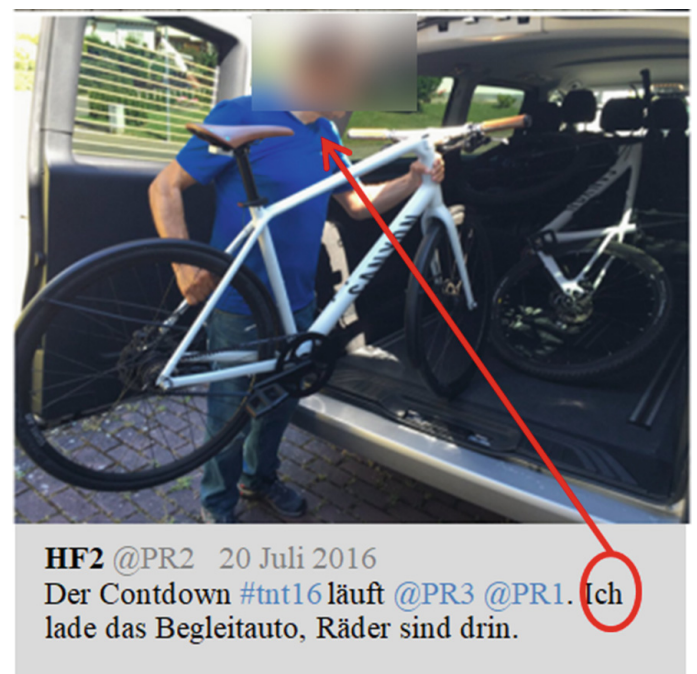

Zusätzlich führt die Person die Handlung aus, die in dem Tweet genannt wird. (2) Eine weitere Möglichkeit ist, dass der/die Autor/in die Funktion der Person benennt. Das setzt voraus, dass andere auf der Fotografie abgebildete Personen bereits in die Erzählung eingeführt wurden und die Rezipient/innen sie erkennen (Abb. 9.26):

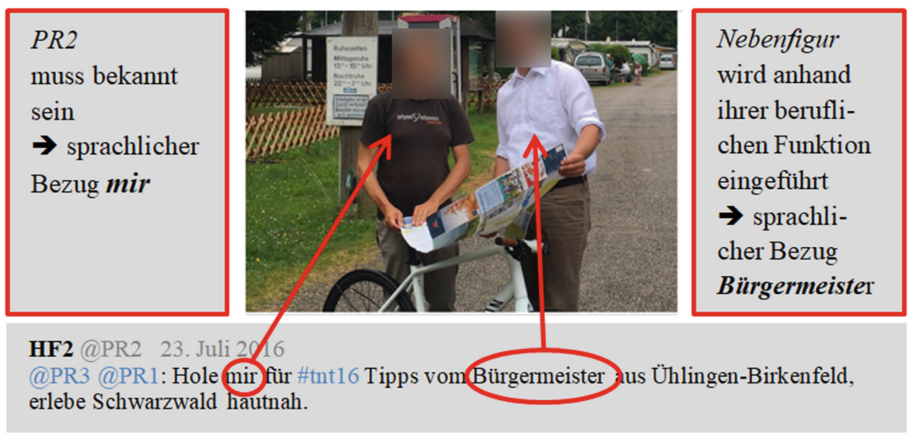

Abb.9.26 Sprachlicher Bezug auf Personen durch Angabe der beruflichen Funktion (PR2, Twitter, 2016-07-23. Korpusquelle: DTKOM_847.2...TW_FB) 
In diesem Tweet müssen die Leser/innen bereits wissen, dass der Mann auf der linken Seite des Fotos einer der Protagonisten (PR2) ist. Ist das bekannt, verbleibt noch eine Person auf der Abbildung, die sprachlich anhand ihrer beruflichen Funktion in die Erzählung eingeführt wird und über das Ausschlussverfahren zugeordnet werden kann. (3) Die dritte Option, auf Personen in Fotografien zu referieren und sie darüber in die Erzählung einzubinden, ist, sie anhand einer Eigenschaft zu markieren. Dabei muss die benannte Eigenschaft mit den visuellen Merkmalen auf der Fotografie übereinstimmen und in den Kontext der Erzählung eingeordnet sein (siehe unten) (Abb. 9.27):

Abb.9.27 Sprachlicher Bezug auf Personen durch Eigenschaftsbenennung (PR2, Twitter, 2016-07-25.

Korpusquelle:

DTKOM_847.14_TW)

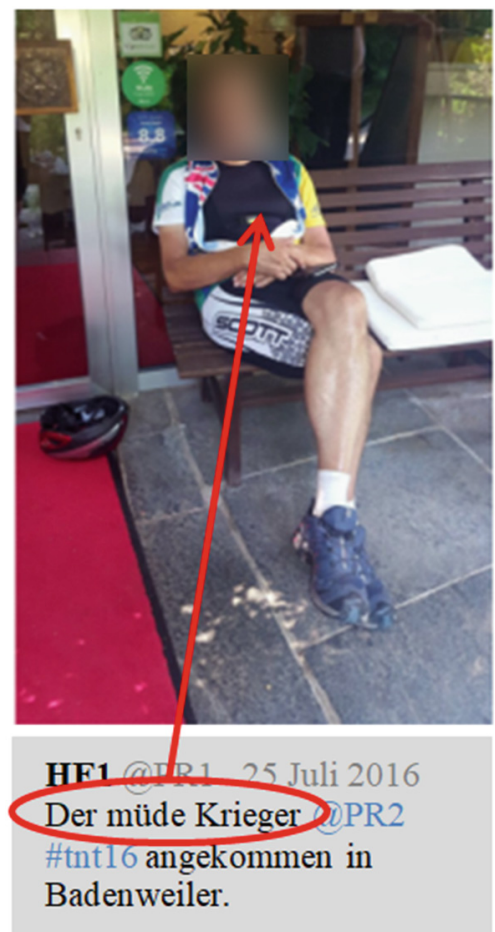

Dieser Tweet stammt aus der Mitte der diskontinuierlichen Erzählung über die Telekom Netz Tour 2016. Bei der abgebildeten Person handelt es sich um PR2, der in zwei Tagen mit dem Fahrrad von der Hochburg in Sexau/Emmendingen 
bis nach Badenweiler radelt. In diesem Erzählkontext muss der Tweet verstanden werden. PR2 sitzt leicht ausgestreckt auf einer Bank. Aufgrund der visuellen Anonymisierung ist nicht mehr erkennbar, dass sein Gesicht glänzt (Schweiß), seine Haare zerzaust sowie die Augen geschwollen und gerötet sind. Diese Merkmale können als bildliche Darstellung des aktuellen Zustands müide gedeutet werden, worüber der Bezug zwischen dem Text und dem Bild hergestellt ist.

In den bisher angeführten Beispielen ging es ausschließlich um den TextBild-Bezug zwischen dem Text und Fotografien mit Personen. Es gibt aber auch textuelle Verweise auf Mensch-Objekt-Konstellationen. Dabei fokussiert der sprachliche Verweis nicht die Person, sondern das Resultat einer von ihr durchgeführten Tätigkeiten. Ist das der Fall, reicht ein Bild nicht aus, um den komplexen Sachverhalt bildlich darzustellen. Die Darstellung einer Tätigkeit bedarf der Dimension ,Zeit', die Bilder nur anhand von sequenzierten Abläufen wiedergeben können. Daher umfassen solche Erzählelemente mindestens zwei Fotografien (Abb. 9.28):

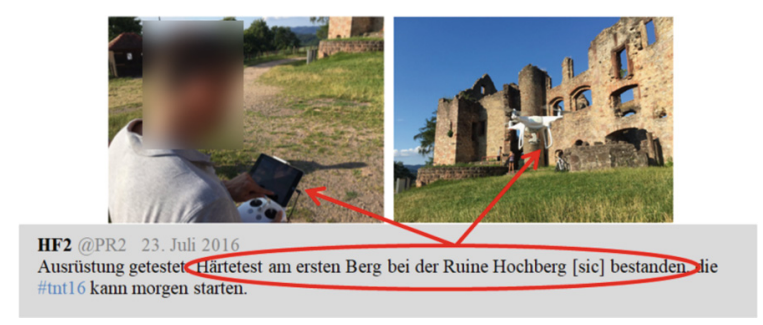

Abb.9.28 Sprachlicher Bezug auf die Fotografie anhand des Resultats einer Tätigkeit (PR2, Twitter, 2016-07-24. Korpusquelle: DTKOM_847.2.2_TW)

Auch dieser Tweet ist nur im Zusammenhang mit der Gesamterzählung nachvollziehbar. Es handelt sich um die erste Station der Telekom Netz Tour 2016. Bei dieser Station üben die Protagonisten den Umgang mit einem Copter (= unbemanntes Flugobjekt mit Fernsteuerung), der im weiteren Verlauf für Überlandbilder eingesetzt und später verunglücken wird. Die Ellipse Härtetest am ersten Berg bei der Ruine Hochberg [sic] bestanden beinhaltet kein Agens, sehr wohl aber das Resultat bestanden. Damit ist implizit eine Tätigkeit verbunden. Da wir wissen, dass ein Agens vorhanden sein muss, stehen zwei Objekte auf der ersten Fotografie zur Auswahl: eine Person und eine Fernsteuerung. Durch das zweite Foto, welches den fliegenden Copter abbildet, kann der Schluss gezogen werden, dass der Copter das Objekt ist, das den Härtetest bestanden hat. Dadurch 
wird im Text auf das Agens im Bild über das Resultat einer Tätigkeit Bezug genommen.

Auch wenn überwiegend Fotografien mit Personen in den erhobenen diskontinuierlichen Erzählungen auftreten, gibt es vereinzelt Text-Bild-Bezüge, die ausschließlich Objekte betreffen. Das Einbinden von Fotografien in die Twittererzählung kann bezogen auf Objekte auf zweierlei Art geschehen. (1) Die Objektbenennung ist eine Möglichkeit, über den Text das bildliche Objekt einzubeziehen. Vorzugsweise setzt der Fotograf die entsprechenden Objekte visuell in den Vordergrund, vergrößert sie oder lichtet sie gleich allein ab (beispielsweise der Copter oben) (Abb. 9.29).

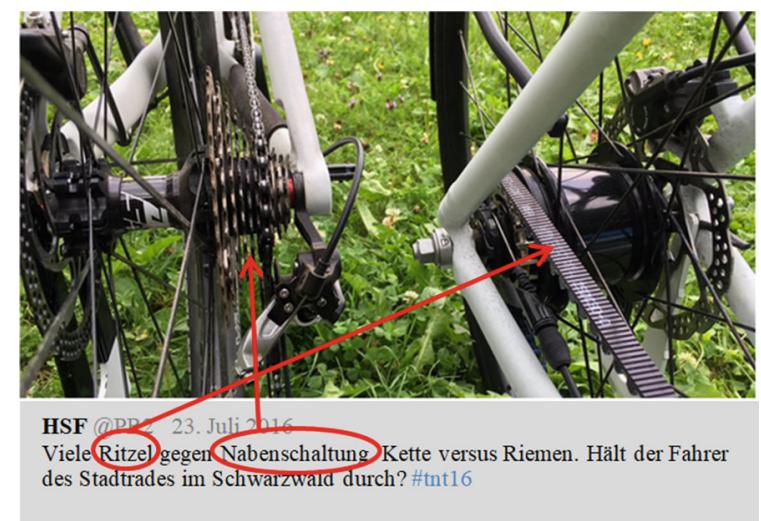

Abb.9.29 Sprachlicher Bezug auf die Fotografie anhand von Objektbenennungen (HF2, Twitter, 2016-07-23. Korpusquelle: DTKOM_847.2.2_TW)

Der Erzähler benennt in seinem Tweet zwei unbelebte Objekte (Ritzel gegen Nabenschaltung der beiden Tourfahrräder). Zumindest die erste Objektbeschreibung ist auch beschreibend, insofern als das Objekt ,Ritzel-Schaltung ' im Bild erkennbar ist. Darüber ist der textuelle Bezug auf das Foto gesichert. Die Gegenüberstellung der beiden Objekte in Verbindung mit der Präposition gegen soll einen Spannungsbogen für den Tourverlauf und das anstehende Rennen etablieren, der sich in der Frage Hält der Fahrer des Stadtrades im Schwarzwald durch? weiter zuspitzt. Die Abbildung in Form einer Gegenüberstellung von den zwei Objekten nimmt den Aspekt der Dramatisierung mit auf. 
Um die zweite Leitfrage zu beantworten, welchen Mehrwert die Fotografien für das narrative Gefüge haben, muss die inhaltliche Informationsverknüpfung von Text und Bild (semantische Verkopplung) in den einzelnen Tweets näher beleuchtet werden. Demnach geht es im Folgenden um Bedeutungskomponenten die das Bild dem Text hinzufügt und deren Beitrag zum Erzähltext. Dazu folge ich Stöckls (2010) Beispiel und expliziere zuerst die Informationsverknüpfungen mithilfe der zwei grundlegenden Muster Elaboration und Extension nach van Leeuwen (2005: 222 ff.). Van Leeuwen (2005) hat in seiner Untersuchung über Informationsverknüpfungen in Texten zwei grundlegende Muster herausgearbeitet und in Unterkategorien spezifiziert. Dazu benennt er jeweils sprachliche Konnektoren zwischen den Informationseinheiten. Für diese Untersuchung sind die sprachlichen Konnektoren ein Hilfsmittel, das mit Paraphrasen Verbindungen zwischen den Textinhalten und den Bildinhalten herstellt. Stöckl merkt zwar an, dass die Unterscheidung der zwei Kategorien „wohl nie trennscharf sein [kann]“ (Stöckl 2010: 58), erkennt aber ihre Bedeutung im Forschungskontext an.

Nach van Leeuwen handelt es sich bei einer verknüpften zweiten Information um eine Elaboration, wenn die zweite Information die erste Information zu Zwecken der Klarstellung wiederholt oder korrigiert (van Leeuwen 2005: 222). Eine elaborierende Information kann auf fünf verschiedene Arten realisiert werden: als Erklärung, Beispiel, Spezifikation, Zusammenfassung und Berichtigung (ebd.). Dabei werden (anders als bei der Extension) keine grundlegend neuen Bedeutungskomplexe oder Wissensbestände hinzugefügt (Stöckl 2010: 58). Bezogen auf die Frage, wie das Bild den Text elaboriert, sind für die vorliegenden Daten das Beispiel, die Erklärung und die Spezifikation relevant.

Findet die Elaboration mithilfe einer visuellen Veranschaulichung statt, ist das mit einer Erläuterung durch ein Beispiel zu vergleichen. Diese zweite Information kann in dem Fall mit den Präpositionalphrasen ,zur Illustration“ oder ,zum Beispiel' angebunden werden. Bezogen auf die Text-Bild-Informationen bedeutet das, dass ein Foto etwas aus dem Text veranschaulicht. Das veranschaulichte Etwas ist dann exemplarisch. Darunter fällt das obige Beispiel, in dem jeweils die Nabenschaltung und die Ritzelschaltung von zwei Fahrrädern zur Illustration abgebildet sind. Der Beitrag für die Erzählung ist darin zu sehen, dass Fotos eine verbale Aussage durch visuelle Merkmale explizieren.

Im Fall einer Erklärung wird in der zweiten Information reorganisiert, was in einer anderen Information bereits gesagt wurde. Einleiten können das die Phrasen ,das ist' oder ,in anderen Worten'. Das Bild, das eine erklärende zweite Information vermittelt, enthält die Inhalte der ersten Information in anderer Gestalt (siehe unten) (Abb. 9.30). 


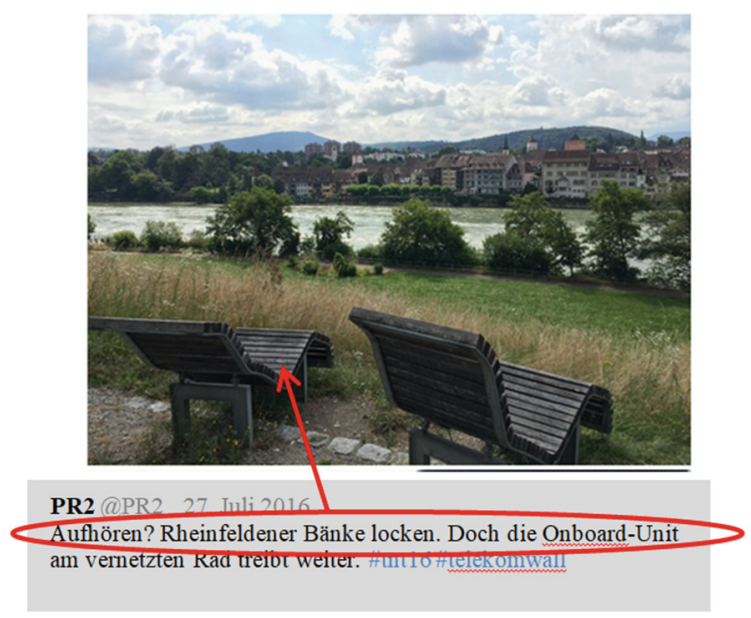

Abb.9.30 Informationsverknüpfung nach dem Muster der Elaboration durch eine Erklärung (PR2, Twitter, 2016-07-27. Korpusquelle: DTKOM_847.2...TW_FB)

In diesem Tweet lässt PR2 seine Rezipient/innen wissen, dass er sich während seiner Radtour gerne etwas ausruhen würde. Das Bild kann mit der Phrase ,in anderen Worten, die geschwungenen Bänke laden zu einer Pause ein“ semantisch angeschlossen werden. Sicher ist dem Bild auch eine Extension inhärent, da auch der Ausblick lockt. Das verdeutlicht, dass für ein Bild nicht unbedingt nur ein Verknüpfungstyp zutreffen muss und die Trennung analytisch motiviert ist. Der Beitrag solcher Fotografien für die Erzählung besteht darin, dass sie den Inhalt einer verbalen Aussage illustrieren und ihn mit persönlichen Eindrücken anreichern, die andernfalls versprachlicht werden müssten. Dabei erfassen sie nicht nur Objekte, sondern auch den Raum und die Atmosphäre.

Der Verknüpfungstyp Spezifikation tritt sicher am häufigsten auf. Das ist dadurch bedingt, dass Fotografien Entitäten fokussieren und ,in Szene“ setzen können. Auf diese Weise spezifizieren sie materiale, räumliche aber auch affektive Aspekte. Diese Bildinformation kann mit der Phrase ,im Speziellen ' oder ,genauer gesagt' angeschlossen werden. Das betrifft beispielsweise den müden Krieger, dessen Fotografie mit der Phrase, im Speziellen sieht ein müder Krieger so aus' angeschlossen weerden kann. Aus der narrativen Perspektive ist die visuelle Spezifizierung des müden Kriegers ein dramaturgisches Moment. 
Auch in dem folgenden Tweet liegt eine Spezifizierung der Person vor, allerdings mit einer anderen Funktion für die Erzählung (Abb. 9.31):

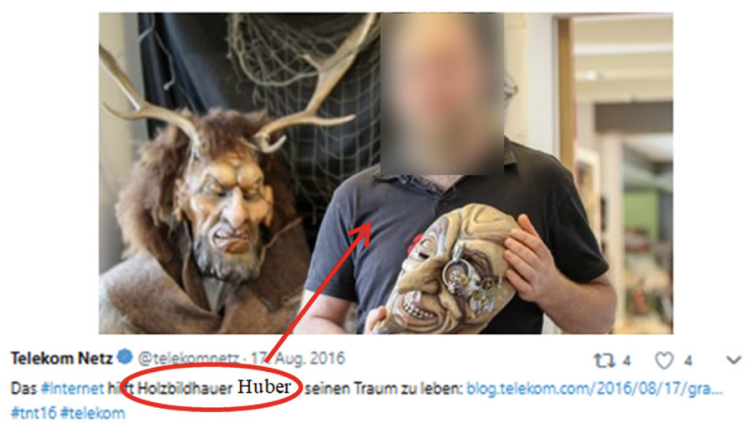

Abb.9.31 Informationsverknüpfung nach dem Muster der Elaboration durch eine Spezifizierung (Deutsche Telekom, Twitter, 2016-08-17. Korpusquelle: DTKOM_847.2...TW_FB)

Dieser Tweet bezieht sich auf eine Tourstation im Schwarzwald. Zum einen spezifiziert das Bild eine affektive Komponente, nämlich, wie man aussehen kann, wenn man seinen Traum lebt (hinter der visuellen Anonymisierung lächelt die Person zufrieden). Zum anderen wird „Holzbildhauer Huber“ durch die Abbildung seiner Person konkretisiert. Wichtig für den narrativen Beitrag von Fotografien ist die Spezifizierung von Personen, da beispielsweise anhand von Kleidung, Werkzeug und Gegenständen Rollen innerhalb der Erzählung zugewiesen werden können.

In einer Informationsverknüpfung nach dem Muster der Extension reichert die zweite Informationseinheit die erste um neue, unbekannte Informationen an (van Leeuwen 2005: 222). Das geschieht nach van Leeuwen durch die einfache Addition von Informationen, wie zusätzliche temporale, logische und räumliche Inhalte. Genau genommen kann jedes Foto ein Mehr an Informationen anbieten. Auch die bereits genannten Beispiele bieten zusätzliche Informationen an. Ich gehe allerdings davon aus, dass in diskontinuierlichen Erzählungen der Text die Bildbetrachtung lenkt. Aus diesem Grund orientiere ich mich an den Mustern der Informationsverknüpfung, die vom Begleittext ausgehend die Bildinhalte anhand von sprachlichen Mitteln mit dem Text verbinden, und erschließe darüber, welchen Beitrag die Fotografien für die Erzählung leisten können. 
Im Fall der diskontinuierlichen Erzählungen dominiert die einfache Addition von Informationen. Es handelt sich um Leerstellen im Text, die sozusagen aus dem Bild erschlossen werden sollen. Welche Informationen das betrifft und wie das umgesetzt ist, zeigen die folgenden Beispiele. Die nächste Abbildung veranschaulicht, wie objektbezogene Informationen durch das Bild hinzugefügt werden können und was sich daraus für ein Beitrag in Bezug auf die Erzählung ergibt (Abb. 9.32):

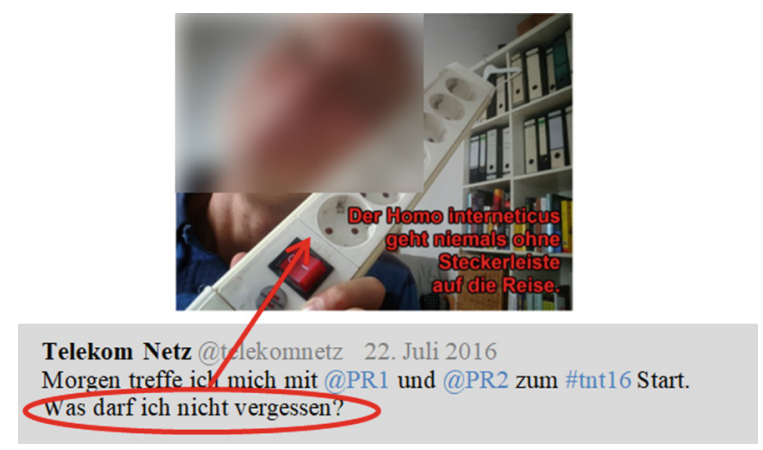

Abb.9.32 Informationsverknüpfung nach dem Muster der Extension durch eine objektbezogene Informationsaddition (Deutsche Telekom, Twitter, 2016-07-22. Korpusquelle: DTKOM_847.2.2_TW)

Die abschließende Frage Was darf ich nicht vergessen? eröffnet eine inhaltliche Leerstelle, die das Bild füllt: nämlich die Steckleiste. Unabhängig von dem integrierten Text, entsteht durch die spielerischen Bezüge zwischen Text und Bild ein kreatives und humorvolles Stimmungsbild. Der Beitrag des Fotos für die Erzählung ist darin zusehen, dass es eine Stimmung/Atmosphäre transportiert, ganz abgesehen davon, dass das Bild den Gegenstand überhaupt erst thematisiert. Die Steckdosenleiste greift der Erzähler darüber hinaus auch am Ende der Gesamterzählung erneut auf, da sie offenbar einen großen Wert für seine Arbeit hat. Sie ist demnach ein Erzählthema.

Eine weitere Möglichkeit Informationen mithilfe einer Fotografie hinzuzufügen, ist die inhaltliche Addition (Abb. 9.33): 


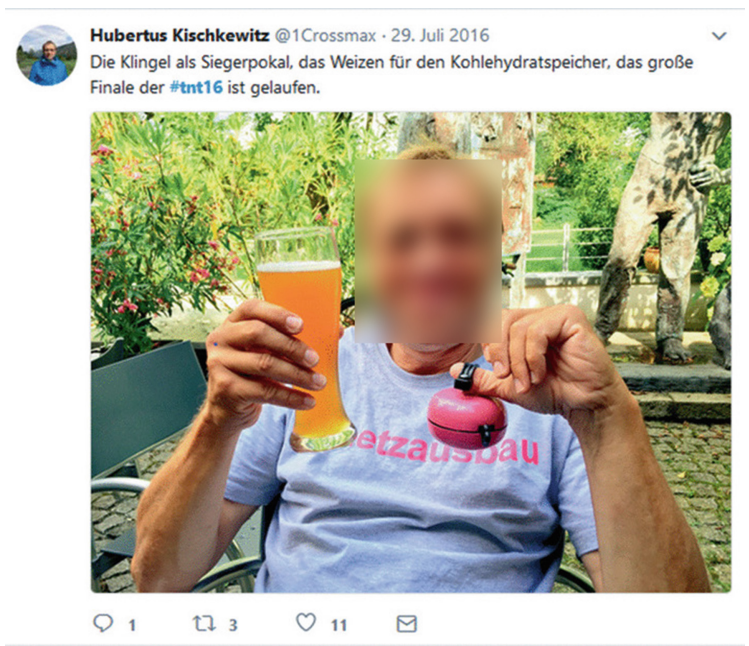

Abb.9.33 Informationsverknüpfung nach dem Muster der Extension durch eine inhaltsbezogene Informationsaddition (Deutsche Telekom, Twitter, 2016-07-29. Korpusquelle: DTKOM_847.2.2_TW)

In diesem Tweet ist das finale Radrennen auf den Feldberg zwischen PR1 und PR2 beendet. Der Tweet verweist anhand der Trophäe (magentafarbene Fahrradklingel) darauf, dass diese dem Sieger gehört. Erst durch das Bild werden wir darüber informiert, welcher der beiden Protagonisten es ist. Im Sinne der Narration übernimmt das Foto die Funktion der Auflösung, indem es die inhaltliche Information, wer gewonnen hat, visualisiert.

Prinzipiell gilt sowohl für die vorhandenen Fotografien der Telekom Netz Tour 2016 als auch für die verbleibenden Daten, dass keine Diskrepanzen zwischen der Fotografie und dem Text auftreten. Eine Diskrepanz zeichnet sich dadurch aus, dass Text und Bild zusammenhangslos nebeneinander stehen (Nöth 2000: 493). Ebenso besteht keine Beziehung der Kontradiktion zwischen semiotischen Ressourcen. In der Kontradiktion widerspricht der Bildinhalt dem Text, wie beispielsweise in einer Karikatur (Nöth 2000: 494). Für Erzählungen im Unternehmenskontext wären diese Beziehungen nicht zielführend, schließlich geht es darum, dass die Rezipient/innen die Tweets möglichst schnell erfassen, und die Fotografien die gewünschte Botschaft unmissverständlich verdeutlichen. Daher bieten sie ein Mehr an Informationen an, kommentieren, emotionalisieren, identifizieren, authentifizieren, charakterisieren und sie vermitteln Eindrücke. Das 
sind komplexe Vorgänge, die sprachlich - insbesondere mit einer Zeichenbeschränkung auf 140 Zeichen - nur schwer zu bewältigen sind. Hinzu kommt, dass in einer Zeit, in der häufig auf Archivbilder zur Illustration zurückgegriffen wird, eine Fotografie des realen Vorgangs mit seinen realen Akteuren, die Einzigartigkeit der Ereignisse unterstreichen soll.

\subsubsection{Bewegtbilder}

In audiovisuellen Erzählfragmenten erhöhen unterschiedliche, kombinierte Zeichenformen (schriftliche/mündliche Sprache, Gestik, Mimik, Geräusche, Musik u. a.) die Komplexität der Erzählung. (Lahn/Meister 2013: 268) Für audiovisuelle Bestandteile in diskontinuierlichen Erzählungen von Unternehmen ist insbesondere die Verknüpfung von Sagen und Zeigen im Bewegtbild von Bedeutung: Die Beweiskraft des Visuellen und die Performanz des/der Erzähler/in werten die Erzählung im Vergleich zu rein schriftlichen Texten und statischen Bildern auf. In den erhobenen Tweets treten zwei mögliche Varianten audiovisueller Einbindungen auf. Bei der ersten Variante handelt es sich um kurze (ca. 30-sekündige), vermutlich mit der Handykamera gefilmte Einschübe $(\mathrm{N}=11)$, in denen Personen sich selbst, ihre Umgebung, andere Personen oder Objekte filmen. Die zweite Variante ist zeitlich umfangreicher (bis max. 10 Min.) und komplexer. Sie wurde für YouTube aufbereitet und in den entsprechenden Tweet eingebunden $(\mathrm{N}=5)$. Im Falle der Telekom Netz Tour 2016 sind das zusammengeführte Ausschnitte des Tagesgeschehens, die entfernt an eine Dokumentation des Tourtages erinnern (ähnlich einem Videotagbuch). Grundsätzlich muss eine filmische Dokumentation nicht immer eine Geschichte erzählen. In erster Linie geht es darum, objektiv auf eine thematisierte Wirklichkeit zu referieren. (Friedmann 2017: 185) Im Fall der eingebundenen YouTube Filme von der NetzTour 2016 setzen die Erzähler jedoch innerhalb des Films spezifische Themenschwerpunkte der gesamten diskontinuierlichen Erzählung narrativ um (z. B. Radrennen, Coptereinsatz). Das Vorkommen zweier verschiedener audiovisueller Darbietungsvarianten resultiert daher, dass die integrierten Bewegtbildausschnitte für die Gesamterzählung sowohl auf der Ebene der Geschichte (histoire) als auch auf der Ebene des Erzählens (discours) funktional sind. Auf diesen Zusammenahng gehe ich in den folgenden drei Beispielen ein.

Das erste Beispiel setzt auf der Ebene der Figurenrede in der Gesamterzählung an. In dem Beispiel schickt PR1 zu Beginn der Telekom Netz Tour 2016 eine „Kampfansage“ an seinen Radrenngegner. Dazu lässt er sich in Manier des Siegessicheren beim genussvollen Essen filmen. In dem einleitenden Twittertext ja, auch ich bereite mich auf die Telekom Netz Tour 2016 vor soll die Diskurskurspartikel $j a$ eine Reaktion und somit eine Interaktion simulieren, die der Sprecher in seiner Bewegtbildbotschaft bezogen auf PR2 und PR3 fortführt: 
(Bsp.) Audiovisuelles Bewegbild zur Gestaltung des Erzählens (discours), erste Darstellungsvariante (PR1, Twitter, 2016-07-19. Korpusquelle: DTKOM_847.2.2_TW_HS3_1)

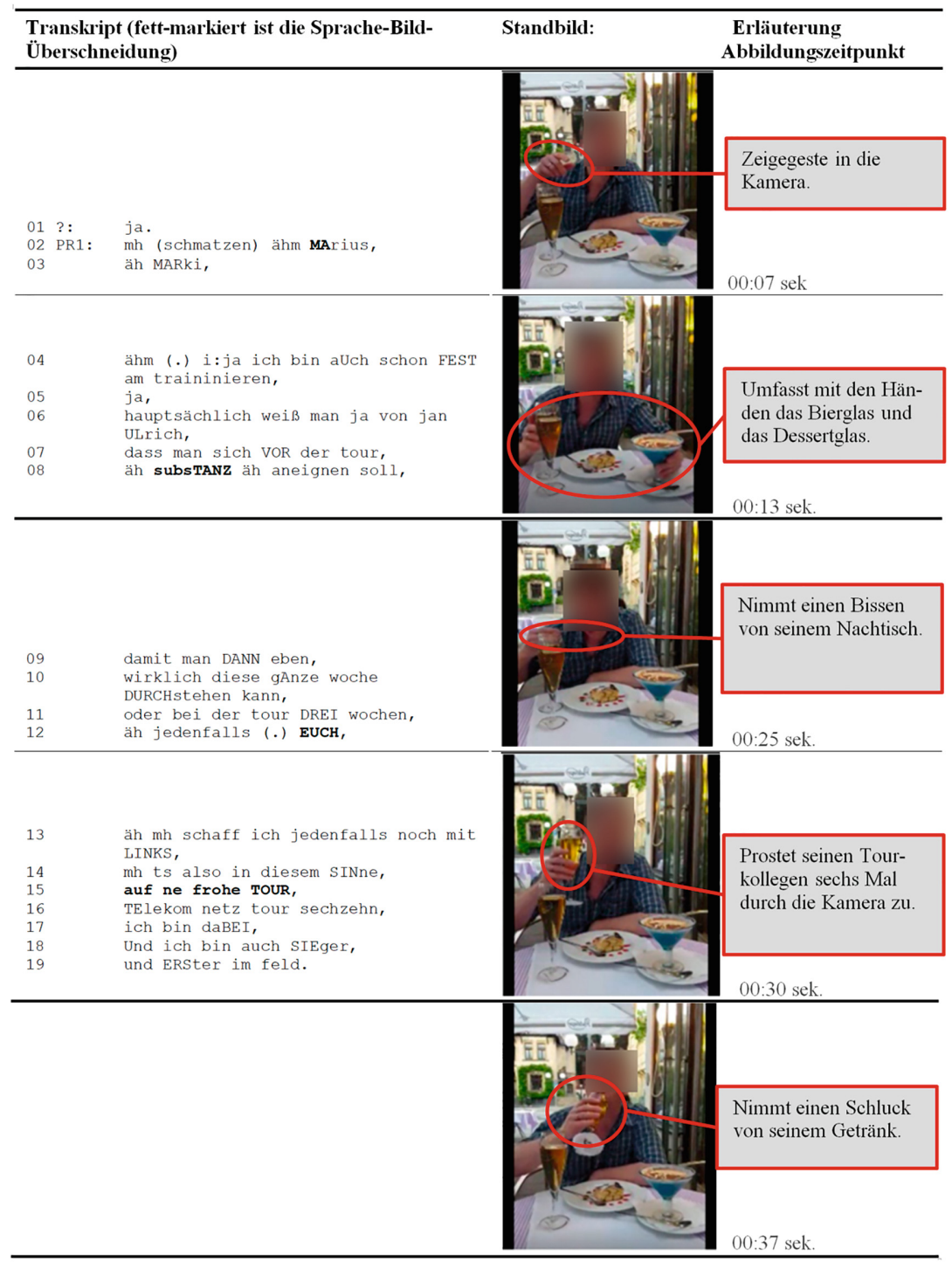


Die Bewegtbildaufnahme ist absichtsvoll inszeniert: PR1 sitzt zu Beginn mit gesenktem Blick frontal vor der Kamera. Auf dem Tisch vor ihm stehen Süßspeisen und zwei gefüllte Biergläser. Zuerst ist eine personell nicht zuordenbare Stimme zu hören. Die Stimme signalisiert mit ja, dass die Kamera aufnimmt. PR1 hebt den Blick in die Kamera und beginnt mit einer Art battletalk, der sich scherzhaft an Kampfansagerituale zwischen Boxsportler/innen vor dem Spiel anlehnt. Mit einer Zeigegeste in Richtung Kamera spricht PR1 seinen Kontrahenten an $\boldsymbol{m h}$ Marius, äh Marki. Danach folgt eine nicht ganz ernst gemeinte Anspielung auf seine Vorbereitungsmaßnahme für das Radrennen. Sie besteht in der Aufnahme von „Substanzen“ - in Anspielung auf den Dopingskandal des Radrennfahrers Jan Ulrich. Die Divergenz zwischen Dopingsubstanzen und Kohlehydraten in Form von Süßspeisen und Bier visualisiert PR1 mit dem Griff zu den Speisen bzw. Getränken. Seine Kampfansage äh jedenfalls (.) EUCH, /äh mh schaff ich jedenfalls noch mit LINKS unterstreicht er mit einem Bissen: Als wolle er sagen ,Euch fresse ich zum Frühstück'. Zusammen mit der Aussage dabei zu sein prostet er dem imaginierten Gegenüber zu. Zum Abschluss kürt er seinen vorausgesagten Sieg (Und ich bin auch SIEger) symbolisch mit einem großen Schluck Bier. PR1 inszeniert absichtsvoll eine künstliche Situation, in deren Kontext er seinen battletalk stellt. Vordergründig adressiert er in seiner Rolle als PR1 seinen Gegner Marius (PR2). Dabei kokettiert er mit einem Kampfansage-Schema, das er scherzhaft inszeniert. Die Anfangssituation, die Utensilien, Gesten und Betonungen sowie Häsitationsphänomene (schmatzen, gefüllte und ungefüllte Pausen) sollen seine Überlegenheit karikieren. Hintergründig soll die Performanz die Rezipient/innen unterhalten und eine gute Grundstimmung für die Tour prognostizieren. Die Funktion des audiovisuellen Beitrags auf der Ebene des Erzählens ist vielfältig: PR1 stellt sich in seiner Rolle vor und charakterisiert sich durch sein Verhalten. Gleichzeitig positioniert er sich zu den anderen Protagonisten und steigert so die Fallhöhe im Sinne der Dramaturgie.

Neben audiovisuellen Bewegtbildern, die auf der Ebene des Erzählens ansetzen, besteht die Möglichkeit, Bewegtbilder im Rahmen der Geschichte zu integrieren. In diesem Fall bezieht sich der einleitende Twittertext Warum ich einen Kasten Küsse? auf die Geschichte in dem Handyfilm (Abb. 9.34).

Der einleitende Twittertext orientiert sich darüber hinaus an einem spezifischen Muster: Der Aussagesatz Das geht ja schon gut los lässt eine Komplikation, etwas Unvorhergesehenes erwarten. Aufgrund der Modalpartikel $j a$ ist der Satz - für sich alleine gelesen - ironisch konnotiert. Die folgende Reformulierung in Form einer Frage (oder „Warum ich einen Kasten Küsse?") löst diese Konnotation dadurch auf, dass die Partikel konzeptionelle Mündlichkeit (Koch/Oesterreicher 1985) indizieren soll. Die textuelle Bezugnahme ähnelt dem Verfahren des 


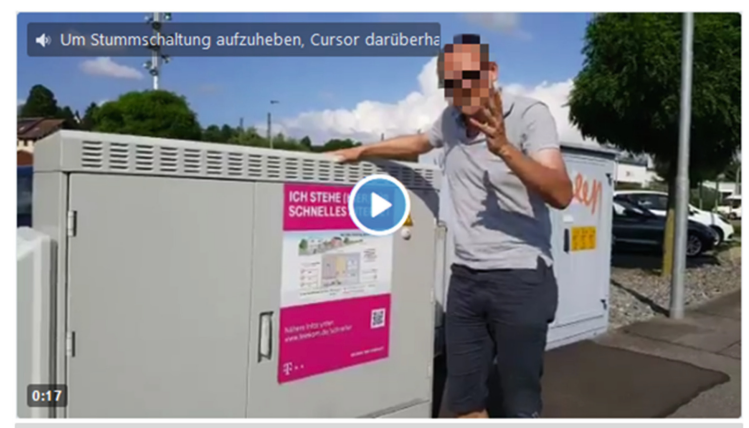

PR3@PR3 24. Juli 2016

Das geht ja schon gut los oder: „Warum ich einen Kasten Küsse?“\#tnt16@telekomnetz \#telekom wall \#emmendingen

Abb. 9.34 Textuelle Bezugnahme auf das audiovisuelle Bewegtbild (PR3, Twitter, 201607-24. Korpusquelle: DTKOM_847.2_TW_HS3_2)

Clickbaiting, das durch aufmerksamkeitsheischende Phrasen ,,auf Neugierde und persönliche Ansprache“ (Primbs 2016: 28) bei den Leser/innen setzt. Das Ereignis, das uns entgegen der evozierten Erwartung in dem Beispiel präsentiert wird, ist aber eher unspektakulär. Erst durch die multimodale Umsetzung wird es sprachlich und gestisch so gestaltet, dass es aufgrund der persönlichen Eindrücke und Empfindungen von PR3 auch für die Rezipient/innen an Bedeutung gewinnt. Wie PR3 das umsetzt, verdeutlicht das folgende multimodale Transkript: 
(Bsp.) Audiovisuelles Bewegtbild zur Gestaltung der Geschichte (histoire), erste Darstellungsvariante (PR3, Twitter, 2016-07-24. Korpusquelle: DTKOM_847.2.1_TW)

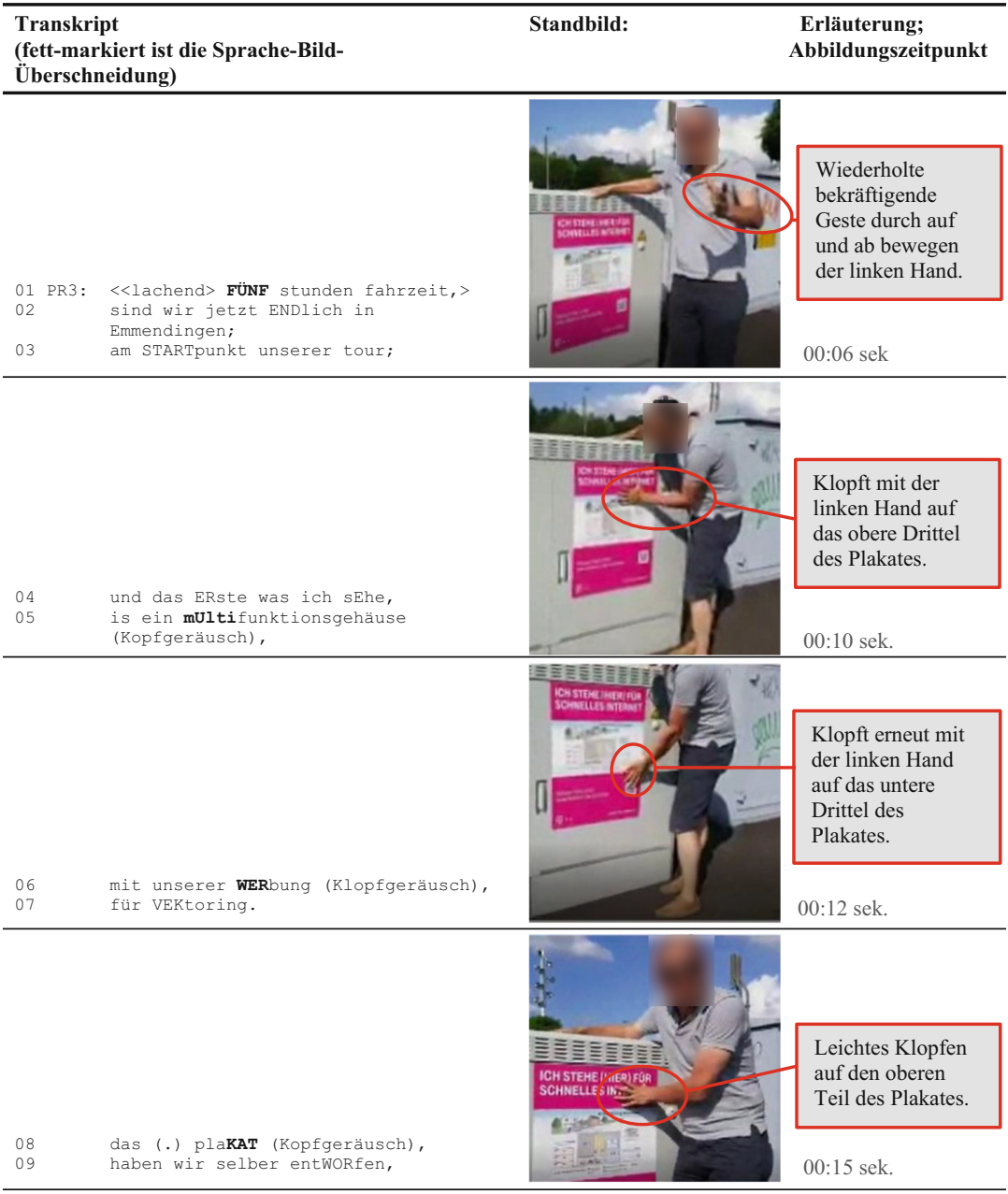




\begin{tabular}{l}
$\begin{array}{l}\text { Transkript } \\
\text { (fett-markiert ist die Sprache-Bild- } \\
\text { Überschneidung) }\end{array}$ \\
\hline $\begin{array}{l}\text { also wIR von der unternehmens- } \\
\text { kommunikation; }\end{array}$
\end{tabular}

Die Perspektive auf das Bewegtbild hat PR3 so eingestellt, dass er frontal zu der Kamera steht. Er blickt direkt in die Kamera, wendet sich dadurch also direkt an die Rezipient/innen und spricht mit leicht bitter-lachendem Ton. Seine auditive Performanz unterstreicht er visuell, indem er die geöffnete Hand schwungvoll auf- und abbewegt. Darüber etabliert er rund um das zunächst banal wirkende Thema ,Plakat' einen Bedeutungsraum. Zeitgleich mit den verbalen Betonungen in Zeile 5 (mUltifunktionsgehäuse), Zeile 6 (WERbung) und Zeile 8 (plaKAT) klopft er mit Nachdruck auf verschiedene Stellen des Plakats. Damit verstärkt er auditiv und gestisch den sprachlichen Ausdruck (gestischer Intensivierer) und lenkt den Blick der Betrachter/innen auf bestimmte Bereiche des Werbeplakats. Das dreimalige Klopfen betont die Bedeutung seiner „Entdeckung“ und ist Ausdruck seiner Begeisterung. Mit der Zeigegeste auf seine Person in Verbindung mit dem Personalpronomen wir stellt er sich nicht nur selbst in den Kreis der kreativen Plakatschöpfer, sondern bringt auch seinen Stolz über das Resultat zum Ausdruck. Dieser gipfelt letztlich in Zeile 11 in einen Emotionsausdruck (ich LIEbe es), den er mit einem Kuss symbolisch besiegelt. Das Bewegtbild hat für die Erzählung demnach eine emotionalisierende und darüber eine beziehungskonstituierende Funktion. Stilistisch schließt es darüber an die konzeptionell mündlich gestaltete textuelle Einleitung des Tweets an. Der Erzähler setzt mithilfe 
seiner Performanz das Ereignis relevant und stiftet Sinn für das unspektakuläre Ereignis (Merkmal der Erfahrungshaftigkeit), welches im Sinne fachlicher Komponenten zur (S)D des Unternehmens beiträgt.

Die letzten zwei Beispiele stammen von nicht bearbeiteten BewegtbildEinspielern in den Tweets (erste Darstellungsvariante), die spontan, aus der Situation heraus gefilmt erscheinen sollen. Eingebundene YouTube-Filme (zweite Darstellungsvariante) sind dagegen inhaltlich dichter, zeitlich umfangreicher und aufwendiger bearbeitet. PR3, der die Filme produziert, schöpft die Möglichkeiten von Bewegtbildern mithilfe weiterer semiotischer Ressourcen aus. Dazu gehört beispielsweise, Landschaftsaufnahmen mit Musik zu unterlegen oder das Bewegtbild durch integrierte Schrift zu kontextualisieren. Die Darstellungselemente sind meist abrupt und ausschnittsweise aneinandergereiht. Kohärenzstiftend ist der Rahmen ,Dokumentation eines Tourtages', der eine Linearität der Abläufe voraussetzt. Exemplarisch erläutere ich einen multimodalen Transkriptausschnitt, der aus einem YouTube-Film vom dritten Tourtag stammt. In dem Ausschnitt geht es um den Copter, der in der diskontinuierlichen Erzählung am 24. Juli eingeführt, am 26 Juli in Aktion vorgeführt wurde und am 27. Juli verunfallte. Dieses „Drama“ bindet PR3 anhand des YouTube Films in die diskontinuierliche Erzählung ein: 
(Bsp.) Audiovisuelles Tourtagebuch auf YouTube, zweite Darstellungsvariante (PR3, YouTube, 2016-07-28. Korpusquelle: DTKOM_847.5_YT)

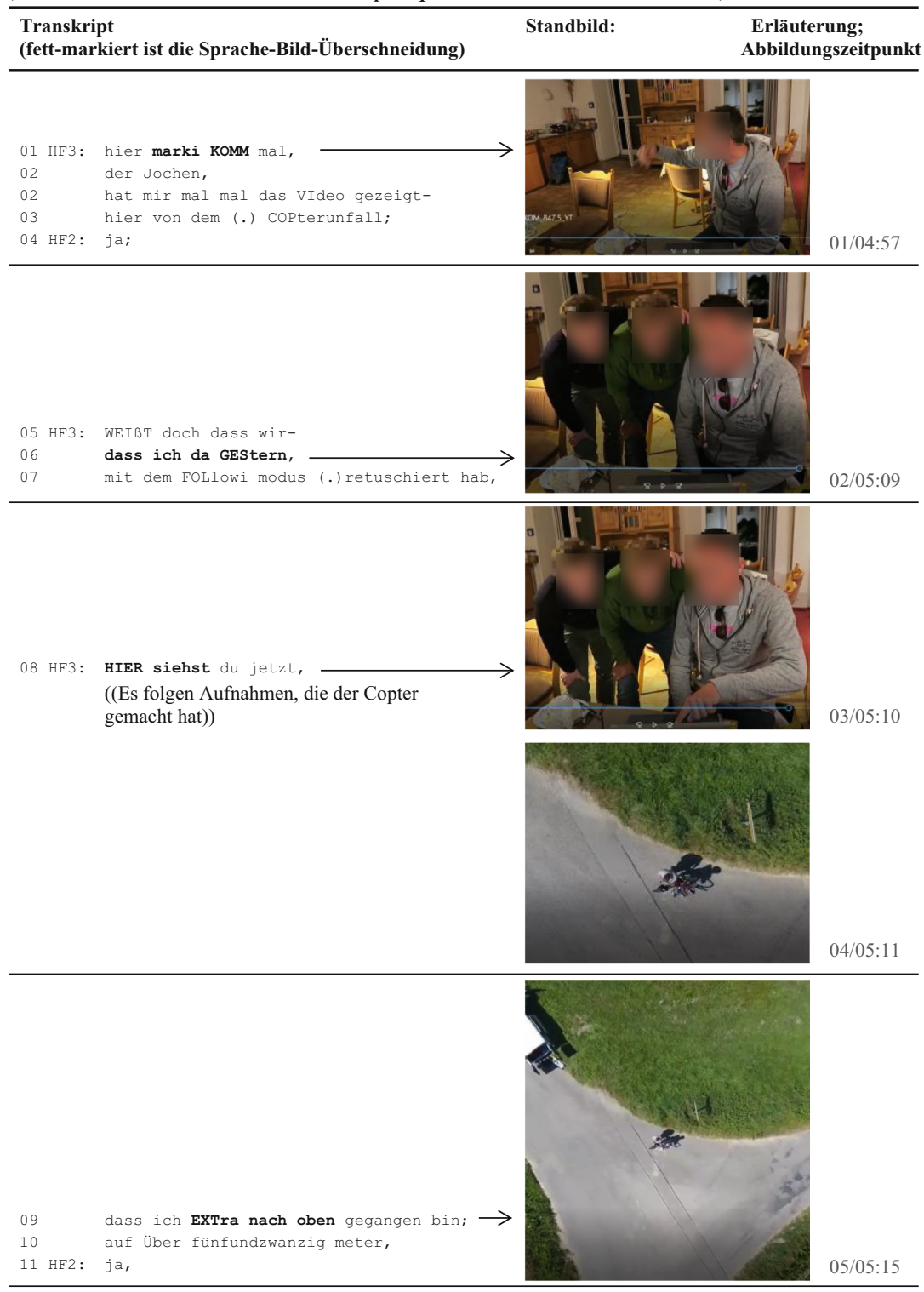




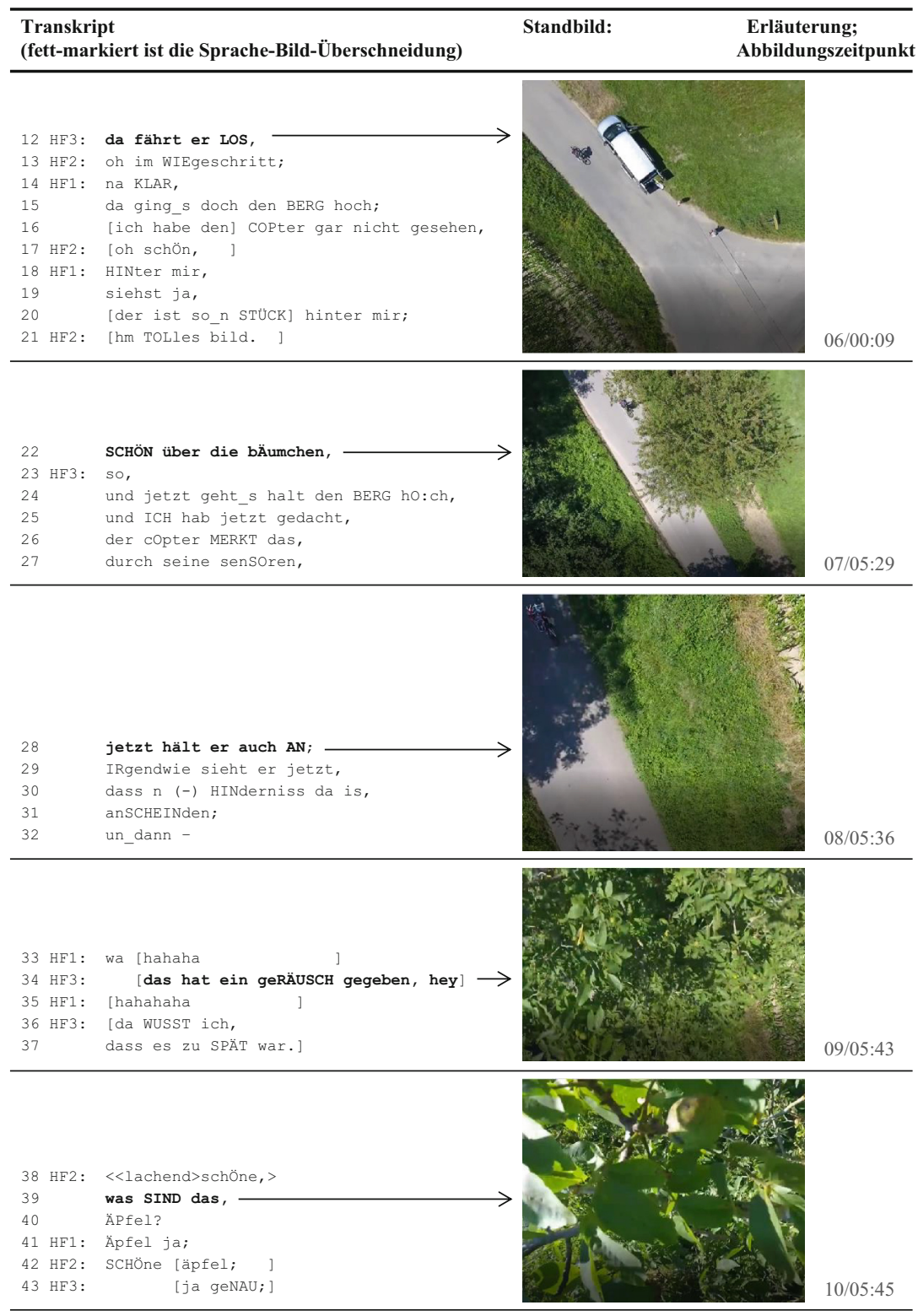




\begin{tabular}{|c|c|c|c|}
\hline \multicolumn{2}{|c|}{$\begin{array}{l}\text { Transkript } \\
\text { (fett-markiert ist die Sprache-Bild-Überschneidung) }\end{array}$} & \multirow[t]{2}{*}{ Standbild: } & $\begin{array}{l}\text { Erläuterung; } \\
\text { Abbildungszeitpunk }\end{array}$ \\
\hline $\begin{array}{l}44 \\
45 \\
46 \text { HF2: } \\
47 \text { HF3: } \\
48 \\
49 \\
50 \text { HF2: } \\
51 \text { HF1: }\end{array}$ & $\begin{array}{l}\text { kuck mal da HäNGT er dann, } \\
\text { bis (.) } \\
\text { hm: } \\
\text { bis en WINDstoß kommt, } \\
\text { des is_n WINDstoß gekommen, } \\
\text { sonst hätt_ma ihn gar nicht beKoMmen; } \\
\text { hat er sich SELBST befreit; } \\
\text { dann haben wir ihn geFUnden, }\end{array}$ & & $11 / 05: 47$ \\
\hline $\begin{array}{l}52 \\
53\end{array}$ & $\begin{array}{l}j a \text { er is uns entGEgen gestürzt; } \\
\text { FuÜgelbruch; }\end{array}$ & & 12/06:00 \\
\hline $\begin{array}{l}54 \text { HF3: } \\
55 \text { HF2: } \\
56 \text { HF3: } \\
57 \\
58 \text { HF2: } \\
59 \text { HF1: } \\
60 \text { HF2: }\end{array}$ & $\begin{array}{l}\text { tja so IS_das heute, } \\
\text { [jahahaha ] } \\
\text { [von je- von] JEder katastrophe, } \\
\text { gibt es ne [AUFnahme.] } \\
\text { [hehe } \quad] \\
\text { Stimmt. [haha }]\end{array}$ & & $13 / 06 \cdot 00$ \\
\hline
\end{tabular}

Der Ausschnitt visualisiert die Erzähler und den Film über den Copter-Unfall. $\mathrm{Zu}$ Beginn sehen die Rezipient/innen, wie PR3 seine Kollegen herbeiwinkt, um ihnen die Filmaufnahmen des Copters zu zeigen (hier von dem (.) COPterunfall, Z. 03). Die drei positionieren sich direkt vor dem Computerbildschirm. Kurz nachdem PR3 mit dem Zeigefinger auf den Bildschirm weist (HIER siehst du jetzt, Z. 08), wechselt das Bewegtbild von den drei Protagonisten auf die originale Copteraufnahme. PR3 erklärt anhand der Bilder, wie er den Copter lenkte (dass ich Extra nach oben gegangen bin, Z. 09) und was auf den Bildern zu sehen ist (da fährt er LOS, Z. 12). PR2 stichelt PR1 wegen seines Fahrstils (oh im WIEgeschritt, Z. 13) und bewertet die Copteraufnahmen (oh schön, Z. 17 / hm TOLles bild, Z. 21), während PR1 kommentiert, dass er den Copter beim Radfahren gar nicht bemerkte. Dadurch überbrücken sie nicht nur die Zeitspanne bis zum „Höhepunkt“ des Films, sondern steigern auch die Spannung. PR3 leitet in Zeile 23 mit der Diskuspartikel so den Wendepunkt ein. Da der Film nicht so schnell abläuft, muss PR3 das Moment verzögern. Das bewerkstelligt er, indem er seine Gedanken in der vergangenen Situation teilt (und ICH hab jetzt gedacht, Z. 25 / der cOpter MERKT das, Z. 26 / durch seine senSOren, Z. 27). Erneut setzt 
PR3 in Zeile 28 zu einer Spannungsauflösung an (jetzt hält er auch AN), die er erneut verzögern muss. Diese Mal sucht er nach einer logischen Erklärung für die Reaktion des Copters. Mit dem bezeichnenden narrativen Anschluss un_dann (Z. 32), der bereits Belustigung bei seinen Kollegen auslöst, hebt PR3 seinen damaligen Eindruck im Unfallgeschehen expressiv hervor (das hat ein geRÄUSCH gegeben, hey, Z. 34 / da WUSST ich, Z. 36/ dass es zu SPÄT war, Z. 37). Erneut erfolgt eine Verzögerung im Erzählfluss, die PR3 überbrückt, indem er kommentiert, was er auf den Bildern sieht. Die sich nun ändernde Bildebene wird sofort genutzt, um das Ereignis erneut zu kommentieren (Kuck mal da HÄNGT er dann, Z. 44). Danach folgt die Auflösung, dass ein Windstoß den Copter aus dem Geäst befreit und dieser in die Wiese stürzt. Als in dem Bild ein Flügel des Copters auftaucht, wird das sogleich als Folge des Unfalls erklärt (FL ̈̈gelbruch, Z. 53). Nun wechselt das Bewegtbild zurück auf die drei Protagonisten vor dem Bildschirm. Während PR3 den Umstand, dass es von dem Unfall Filmaufnahmen gibt, scherzhaft bewertet (vonje-von JEder katastrophe, Z. 56 / gibtes ne AUFnahme, Z. 57), beantworten das seine Kollegen mit Lachen. Die multimodale Präsentation entlang der originalen Copteraufnahmen produziert Längen in der Erzählung, die die Protagonisten nutzen, um die Spannung zu steigern und den Vorgang persönlich zu gestalten. Bezeichnend für die multimodale Repräsentation ist, dass die Erzähler den Unfall anhand der Kameraaufnahmen des Copters reinszenieren, indem sie die Aufnahmen kommentieren. Sie schildern dabei ihre Eindrücke, Gedanken und Meinungen, während die Copteraufnahme das unmittelbare Geschehen in Echtzeit visualisiert. Dadurch sind die Zeit des Geschehens und der Rezeption kongruent. Zusammen mit dem szenischen Präsens und der Kameraaufnahme in Echtzeit entsteht die Illusion der direkten Teilhabe am Geschehen (Involvierungsstrategie). Insgesamt erhält die Erzählung durch die multimodale Präsentation ein Mehr an Informationen und Eindrücken. Der YouTube-Filmausschnitt unterscheidet sich von den ersten zwei Beispielen darin, dass er nachbearbeitet ist und zwei Kameraperspektiven von unterschiedlichen Aufnahmegeräten zusammenführt. Gemeinsam ist den vorgeführten multimodalen Realisierungen, dass sie die Ebenen ,Sagen und Zeigen“ mithilfe verschiedener semiotischer Ressourcen verbinden. 


\subsubsection{Erzähltheoretische Einordnung}

Beziehe ich die herausgearbeiteten Merkmale diskontinuierlicher Erzählungen auf die an Small Stories adaptierten narrativen Dimensionen von Page - wie zuvor bei den (bimodalen) Kompakterzählungen in Abschn. 9.3.5 - zeigen sich Gemeinsamkeiten und Unterschiede zwischen den beiden Realisierungstypen. Mithilfe dieses Schrittes lassen sich spezifische Merkmale von diskontinuierlichen Erzählungen anschaulich explizieren. In der folgenden Abbildung indizieren die Kreuze, zu welchem Pol eines narrativen Merkmals diskontinuierliche Erzählungen tendieren (Abb. 9.35):

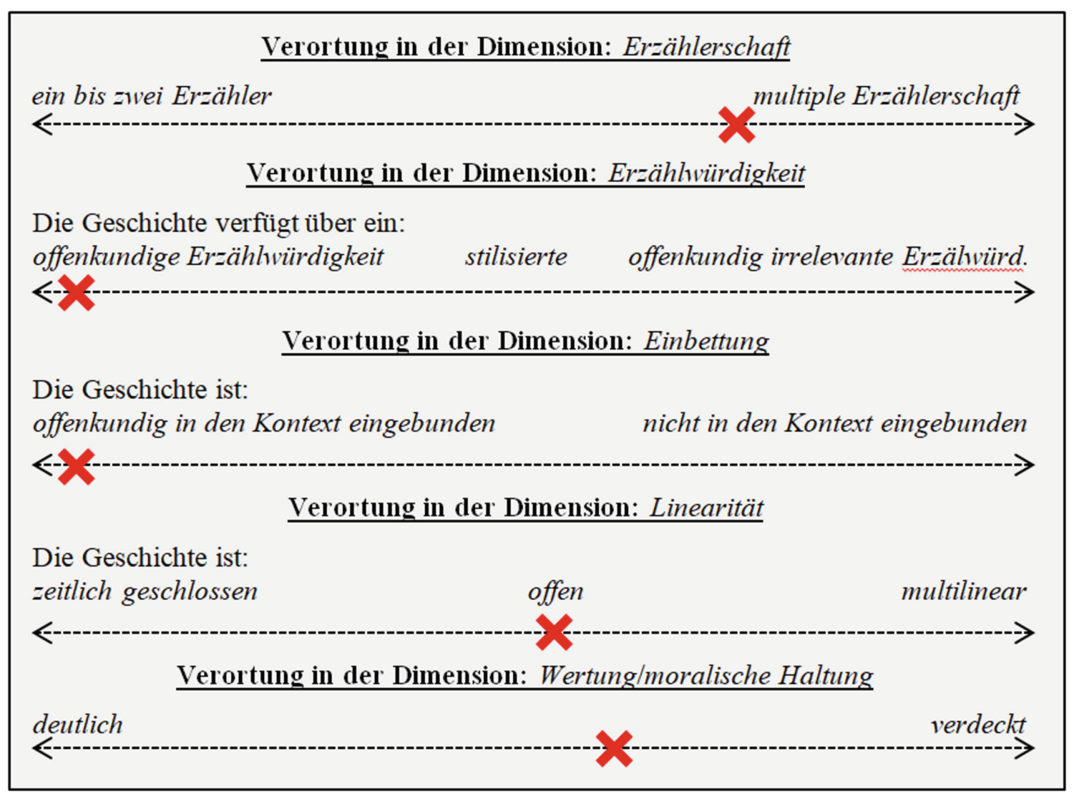

Abb.9.35 Skalare Pol-Tendenzen von diskontinuierlichen Erzählungen innerhalb der narrativen Dimensionen für Erzählungen im Internet (angelehnt an Ochs/Capps 2001: 22 ff., inhaltlich nach Page 2015: 333) 
Die Dimension ,Erzählerschaft‘ ist für diskontinuierliche Erzählungen schwer zu fassen. Die Wahrscheinlichkeit, dass mehrere Erzähler/innen vorliegen (Erzählerkollektiv), liegt aufgrund der Diskontinuität der Erzählung nahe. Auch die Tatsache, dass Filme von YouTube in die Erzählungen eingebunden werden, indiziert, dass mehrere Autor/innen am Werk sind. In diskontinuierlichen Erzählungen ist das eher erwartbar als in einer Kompakterzählung, bei der es sich um eine geschlossene, kurze Einzelgeschichte handelt. Die Erzählwürdigkeit tendiert deutlich stärker als in Kompakterzählungen zum Pol einer offenkundigen Erzählwürdigkeit, da in 82 Prozent der erhobenen Daten Ereignisse aus dem Themenfeld ,Aktuelle Informationen und Öffentlichkeitsarbeit' den Erzählanlass ausmachen. Gemeinsam ist sowohl Kompakterzählungen als auch diskontinuierlichen Erzählungen, dass sie ebenfalls mithilfe von Symbolen, Firmennamen u. a. in den Unternehmenskontext eingebunden sind. Anders sieht das für die Dimension der Linearität aus. Die Elemente von diskontinuierlichen Erzählungen sind nicht immer geschlossen. Ebenso ist es möglich, dass auch die Gesamterzählung offen bleibt. Meist aus dem einfachen Grund, dass kein abschließendes Erzählfragment gepostet wird, da die Erzähler/innen die Geschichte offensichtlich als Ganzes nicht weiter vorangetrieben haben. Während in einer Kompakterzählung die Wertung/ moralische Haltung der Erzähler/innen zu dem Pol ,deutlich erkennbar ‘ tendiert (insbesondere durch affektive Bewertungen), ist sie in diskontinuierlichen Erzählungen eher verdeckt. Die Bewertung soll stärker von ausgewählten unternehmensbezogenen Handlungen rund um ein Ereignis getragen werden und weniger von den erzählenden Stellvertreter/innen. Welchen Umfang - bemessen an den Erzählfragmenten - eine diskontinuierliche Erzählung aufweist, variiert deutlich. In den Daten rangieren die Erzählungen aus mindestens drei bis maximal 40 Erzählfragmenten zusammen. Ich bin mir bewusst, dass eine diskontinuierliche Erzählung mit 40 Erzählfragmenten nicht unbedingt als „klein“ bezeichnet werden kann. Ihre Bestandteile - also die Erzählelemente/-fragmente jedoch schon. Würde ich die Anzahl der Elemente als Differenzierungskriterium ansetzen, entspräche eine diskontinuierliche Erzählung mit drei Elementen einer Small Story Variante, während jene mit mehreren Elementen, obwohl ihre Elemente die selben Merkmale tragen, aus dem narrativen Vermittlungsvorkommen herausfallen würden. 
Open Access Dieses Kapitel wird unter der Creative Commons Namensnennung 4.0 International Lizenz (http://creativecommons.org/licenses/by/4.0/deed.de) veröffentlicht, welche die Nutzung, Vervielfältigung, Bearbeitung, Verbreitung und Wiedergabe in jeglichem Medium und Format erlaubt, sofern Sie den/die ursprünglichen Autor(en) und die Quelle ordnungsgemäß nennen, einen Link zur Creative Commons Lizenz beifügen und angeben, ob Änderungen vorgenommen wurden.

Die in diesem Kapitel enthaltenen Bilder und sonstiges Drittmaterial unterliegen ebenfalls der genannten Creative Commons Lizenz, sofern sich aus der Abbildungslegende nichts anderes ergibt. Sofern das betreffende Material nicht unter der genannten Creative Commons Lizenz steht und die betreffende Handlung nicht nach gesetzlichen Vorschriften erlaubt ist, ist für die oben aufgeführten Weiterverwendungen des Materials die Einwilligung des jeweiligen Rechteinhabers einzuholen.

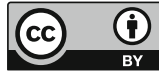

